\title{
From Chromatin Readers To Neuronal Networks: Finding New Treatments For Alzheimer's Disease A Transcriptomics Approach
}

\section{Dissertation}

\author{
for the award of the degree \\ "Doctor rerum naturalium" \\ of the Georg-August-Universität Göttingen \\ within the doctoral program Systems Neuroscience, GGNB \\ of the Georg-August University School of Science (GAUSS)
}

\author{
submitted by \\ Hendrik Urbanke \\ born in Nürnberg, Germany
}

Göttingen 2017 


\section{Thesis Committee}

1. Prof. Dr. André Fischer, Epigenetics und Systems medicine in Neurodegenerative Diseases, German Center for Neurodegenerative Diseases

2. Prof. Dr. Tiago Fleming Outeiro, Neurodegeneration and Neurorestoration, University Medical Center, Göttingen

3. Prof. Dr. Siegrid Löwel, Systems Neuroscience, Zoology and Anthropology, Georg-AugustUniversität, Göttingen

\section{Members of the Examination Board}

Referee: Prof. Dr. André Fischer, Epigenetics und Systems medicine in Neurodegenerative Diseases, German Center for Neurodegenerative Diseases

$2^{\text {nd }}$ Referee: Prof. Dr. Tiago Fleming Outeiro, Neurodegeneration and Neurorestoration, University Medical Center, Göttingen

Prof. Dr. Siegrid Löwel, Systems Neuroscience, Zoology and Anthropology, Georg-AugustUniversität, Göttingen

Prof. Dr. Ralf Heinrich, Cellular Neurobiology, Georg-August-Universität, Göttingen

Prof. Dr. André Fiala, Behavioural Molecular Neurobiology, Georg-August-Universität, Göttingen

Prof. Dr. Martin Göpfert, Cellular Neurobiology, Georg-August-Universität, Göttingen

Date of oral examination: 19.02 .18 
Declaration

I hereby declare that I have written the dissertation

"From Chromatin Readers to Neuronal Networks:

Finding Treatments for Alzheimer's Disease

A Transcriptomics Approach"

independently with no other aids or sources than stated.

Hendrik Urbanke Göttingen, 20.12.17 


\section{Table of contents}

$\begin{array}{ll}\text { List of Figures } & 7\end{array}$

List of Tables $\quad 8$

List of Abbreviations $\quad 9$

1. Introduction 11

1.1. Alzheimer's Disease 11

1.2. Molecular hallmarks of Alzheimer's Disease 12

1.2.1. Amyloid precursor protein and amyloid $\beta \quad 12$

$\begin{array}{ll}\text { 1.2.2. The microtubule associated protein Tau } & 14\end{array}$

$\begin{array}{ll}\text { 1.3. Learning and Memory } & 17\end{array}$

$\begin{array}{lr}\text { 1.3.1. The hippocampus in learning and memory } & 18\end{array}$

$\begin{array}{ll}\text { 1.3.2. Persistence of memory } & 19\end{array}$

$\begin{array}{ll}\text { 1.3.3. Association of memory } & 21\end{array}$

1.3.1. Molecular mechanisms of learning 22

1.4. Epigenetics 24

$\begin{array}{ll}\text { 1.4.1. Chromatin plasticity } & 25\end{array}$

1.4.2. HDACs in learning and memory $\quad 27$

1.4.3. The Readers of the Histone Code $\quad 29$

1.4.4. On chromatin assisted alternative splicing $\quad 30$

1.5. Annexins 30

2. Objectives 35

3. Material and Methods 38

3.1. Animals 38

3.1.1. Animal welfare 38

3.1.2. Thy1.2-Tau22 x HDAC6-/- transgenic mice 38

3.1.3. AnxA2 null mutant mice 39

3.1.4. Genotyping 39

3.1.5. Intracranial Injections $\quad 40$

3.2. Behaviour 41

3.2.1. Behavioural testing 41

3.2.1.1. Open field test 41

3.2.2. Intracardial perfusion 44

3.3. Molecular cloning $\quad 44$

3.3.1. Molecular cloning of AnxA2 44

3.3.2. Bacterial transformation 45

3.3.3. Plasmid isolation $\quad 45$

3.3.4. Adeno-associated-viral particle (AAV) production 45

3.4. Cell culture 46

3.4.1. Dissociated primary neuronal culture 46 
3.4.2.Chemical neuronal stimulation

$\begin{array}{ll}\text { 3.4.3. Dissociated primary neuronal SILAC culture } & 47\end{array}$

$\begin{array}{ll}\text { 3.4.4. Heterogenous primary neuronal cultures } & 47\end{array}$

3.4.5. Optogenetic stimulation 48

$\begin{array}{ll}\text { 3.4.6. Single cell sorting } & 48\end{array}$

3.4.7. Anle138b treatment 49

3.4.8. JS28 treatment 49

3.4.9. Metabolic rate assay $\quad 49$

3.4.10. Membrane integrity assay 49

3.5. Molecular Analysis $\quad 50$

3.5.1. In-silico oligo nucleotide design 50

3.5.2. Total protein extraction $\quad 50$

3.5.3. Nuclear protein enrichment 50

3.5.4. Sub-cellular fractionation $\quad 50$

3.5.1. SILAC nuclear protein isolation $\quad 51$

3.5.2. Immunohistochemistry 51

3.5.3. Immunocytochemistry 52

3.5.4. Confocal imaging 52

3.5.5. Western blotting 52

3.5.6. RNA isolation 53

3.5.7. cDNA generation 53

3.5.8. Quantitative real time PCR 53

3.5.9. RNA sequencing 55

3.5.10. RNAs sequencing data analysis $\quad 55$

3.5.11. Differential gene expression analysis $\quad 55$

3.5.12. Gene Set enrichment analysis 56

3.5.13. Differential exon usage 56

4. Results $\mathbf{5 8}$

4.1. Annexin $A 2$ is a reader of acetylated $H 4 \mathrm{~K} 12$ and impacts spatial memory 58

4.1.1. Annexin $\mathrm{A} 2$ is a reader of $\mathrm{H} 4 \mathrm{~K} 12 \mathrm{ac}$ and is ubiquitously expressed in brain regions associated with learning and memory $\quad 58$

4.1.2. Translation and localisation of ANXA2 change in ageing 60

4.1.3. AnxA2 overexpression impairs hippocampus-dependent spatial memory 62

4.1.4. ANXA2 null mutants display facilitated spatial memory 67

4.1.5. ANXA2 affects the cellular membrane and RNA processing in vivo 70

4.1.6. Neurons respond to ANXA2 overexpression by differential regulation of genes involved in developmental processes and stimulus response $\quad 71$

4.1.7. ANXA2 is involved in ECM and stimulus response processes 73

4.1.8. NMDA but not $\mathrm{KCl}$ treatment changes exon usage in WT hippocampal neurons 76

4.1.9. ANXA2 overexpression and deficiency show opposing effects on exon usage and target pathways crucial for neuronal signal transduction $\quad 77$

4.1.1. ANXA2 manipulation changes NMDA-dependent splicing response 79 
4.2.1. Targeting the interaction between TAU and HDAC 6 to ameliorate AD-like tauopathy

4.2.1."The diphenylpyrazol compound anle138b blocks $A \beta$ channels and rescues disease phenotypes in a mouse model for amyloid pathology"

4.3. Analysing neuronal network dynamics in primary hippocampal cultures 92

4.3.1. Efficient composition of neuronal networks with multiple mutations 92

4.3.2. Introducing the Luminous Channelrhodopsin Inducer For Research (LuCIFR) 94

5. Discussion

5.1. ANXA2- a novel reader of $\mathrm{H} 4 \mathrm{~K} 12 \mathrm{ac}$

5.1.1. ANXA2 reads the histone code in the mouse brain 99

5.1.2. AnXA2 overexpression impairs of spatial memory 102

5.1.3. ANXA2 acts from the extracellular matrix to the nucleus 104

5.1.4. ANXA2 orchestrates RNA modifications that correlate to the H4K12ac signature 105

5.1.5. A side note on ANXA2 life-long deficiency in learning and memory

5.2. HDAC6 modulation and Anle138b: two convergent strategies as novel therapeutic $\begin{array}{ll}\text { avenues to treat } A D & 109\end{array}$

5.2.1. HDAC6 deficiency rescues anxiety in the THY-Tau22 mouse model for AD 110

5.2.1. Anle138b rescues LTP and ameliorates spatial memory deficits in APP/PS1 mouse model for $A D$

5.3. Illuminating neuronal network dynamics 113

6. Summary 117

$\begin{array}{lr}\text { Appendix } & 120\end{array}$

Bibliography 142

$\begin{array}{ll}\text { Acknowledgements } & 169\end{array}$

$\begin{array}{ll}\text { Curriculum Vitae } & 170\end{array}$ 


\section{List of Figures}

Figure 1.1: Alzheimer disease in the United States (2010-2050) estimated using the 2010 consensus. 11

Figure 1.2: Processing of the amyloid precursor protein. 13

Figure 1.3: Aggregation of the microtubule associated protein tau. $\quad 15$

$\begin{array}{lr}\text { Figure 1.4: A possible memory taxonomy. } & 17\end{array}$

$\begin{array}{lr}\text { Figure 1.5: The Hippocampus. } & 18\end{array}$

Figure 1.6: Time dependent stages of memory. $\quad 20$

Figure 1.7: The epigenetic landscape. $\quad 25$

Figure 1.8: The counteracting function of writers and easers. $\quad 27$

Figure 1.9: Chromatin readers read the histone code. 28

Figure 3.1: Generation of genetic background with double transgene. 38

Figure 3.2: Illustration of AAV-6P-AnxA2GFP-NoTB-SEWB. 44

Figure 3.3: Production of heterogenous PNC. 48

Figure 4.1: ANXA2 is expressed the brain and binds selectively acetylated H4K12ac. 59

Figure 4.2: Protein levels of ANXA2 are reduced in CA1 and increased in nuclei of DG in aged mice. 61

Figure 4.3: Expression of ANXA2 in cytosol and nucleus using AAVs. 63

Figure 4.4: Behavioural testing of ANXA2 overexpressing mice shows influence of ANXA2 on 64 cognitive function.

Figure 4.5: Spatial memory is impaired in mice overexpressing ANXA2 in hippocampal DG neurons. 66

Figure 4.6: ANXA2 null mutants show enhanced performance in spatial memory. 68

Figure 4.7: ANXA2 null mutants significantly outperformed WT in the Barnes Maze. 69

Figure 4.8: Gene set enrichment analysis reveals dual involvement of ANXA2 in membrane and RNA 71 processing.

Figure 4.9: ANXA2 overexpressing neurons show wide but mild changes in gene expression and 72 react to NMDA stimulation in an ECM-dependent manner

Figure 4.10: ANXA2 deficiency alters the ECM and susceptibility to stimulation. 75

Figure 4.11: NMDA but not $\mathrm{KCl}$ treatment induces alternative exon usage in primary neuronal culture. 77

Figure 4.12: Differential exon usage between ANXA2 overexpression and deficiency is inverted and 78 targets structural genes associated with neuronal projection.

Figure 4.13: NMDA stimulation of AnxA2 overexpressing or deficient neurons changes exon usage in 80 genes relevant to synaptic plasticity.

Figure 4.14: ANXA2 regulates energy metabolism by alternative 5' usage in an H4K12ac associated 82 manner.

Figure 4.15: JS28 does not change tubular acetylation in PNC.

Figure 4.16: HDAC6 deficiency mildly modulates Tau pathology at 6 months of age (early-stage).

Figure 4.17: Hdac6 KO mildly modulates Tau pathology at 6 months of age (early-stage). 84

Figure 4.18: Hdac6 KO rescues Tau anxiety phenotype at 13 months of age. 88

Figure 4.19: Anle138b ameliorates $A \beta 1-40$ induced membrane permeability. 91

Figure 4.20: Production of heterogeneous neuronal cultures. 93

Figure 4.21: LuCIFR a new optogenetic stimulator for molecular biology. 96

Figure 4.22: A model of the multifactorial chromatin reader ANXA2 in neurons including relevant 108 literature. 


\section{List of Tables}

Table 3.1: Primers used for cloning, genotyping and pPCR 39

$\begin{array}{ll}\text { Table 3.2: Genotyping PCR Protocol Tau22 } & 40\end{array}$

$\begin{array}{ll}\text { Table 3.3: Genotyping PCR HDAC6 } & 40\end{array}$

$\begin{array}{ll}\text { Table 3.4: Genotyping PCR NEO casette } & 40\end{array}$

Table 3.5: Genotyping PCR AnxA2 40

Table 3.6: cDNA synthesis reagents $\quad 54$

Table 3.7: cDNA synthesis incubation $\quad 54$

Table 3.8: Sybr Green qPCR protocol $\quad 54$

Table 3.9: Sybr Green reagent composition $\quad 54$

Table 3.10: UPL Master Probe qPCR protocol $\quad 54$

Table 3.11: UPL reagent composition $\quad 54$

Appendix Table 1: Top 10\% TSS proximity exons differentially expressed in NMDA treated KO 122 vs. WT 


\section{List of Abbreviations}

\begin{tabular}{|c|c|}
\hline$A D$ & Alzheimer's Disease \\
\hline ANXA2 & Annexin $\mathrm{A} 2$ protein \\
\hline AnxA2 & Annexin A2 gene/mRNA \\
\hline APP & Amyloid precursor protein \\
\hline APP/PS1 & C57BI6j-Tg(Thy1-APPsew;Thy1-PS1 L166P) \\
\hline$A \beta$ & Amyloid beta \\
\hline $\mathrm{BP}$ & Biological process \\
\hline CA1 & Cornus ammonis 1 (hippocampal sub-region) \\
\hline ChIPseq & Chromatin immunoprecipitation sequencing \\
\hline CNS & Central nervous system \\
\hline DE & Differential expression \\
\hline DEX & Differential exon usage \\
\hline $\mathrm{DG}$ & Dentate gyrus (hippocampal sub-region) \\
\hline DIV & Day in vitro \\
\hline DNA & Deoxyribonucleic acid \\
\hline GO & Gene Ontology \\
\hline GSEA & Gene set enrichment analysis \\
\hline HAT & Histone acetyltransferase \\
\hline HDAC & Histone deacetylase \\
\hline HDAC6 & Histone deacetylase 6 \\
\hline ISI & Inter stimulation interval \\
\hline KEGG & Kyoto Encyclopedia of Genes and Genomes \\
\hline LTD & Long-term potentiation \\
\hline LTM & Long term memory \\
\hline LTP & Long-term depression \\
\hline MF & Molecular function \\
\hline MTT & 3-(4,5-dimethylthiazol-2-yl)-2,5-diphenyltetrazolium bromide \\
\hline NGS & Next generation sequencing \\
\hline NMDA & N-Methyl-D-aspartic acid \\
\hline PTM & Post-translational modification \\
\hline qPCR & Quantitative polymerase chain reaction \\
\hline RNAseq & Ribonucleic acid sequencing (HiSeq 2000) \\
\hline STM & Short term memory \\
\hline Tau & C57BI6j-TG(THY1.2-Tau22) \\
\hline TAU & Microtubule associated protein tau \\
\hline TSS & Transcription start site \\
\hline
\end{tabular}




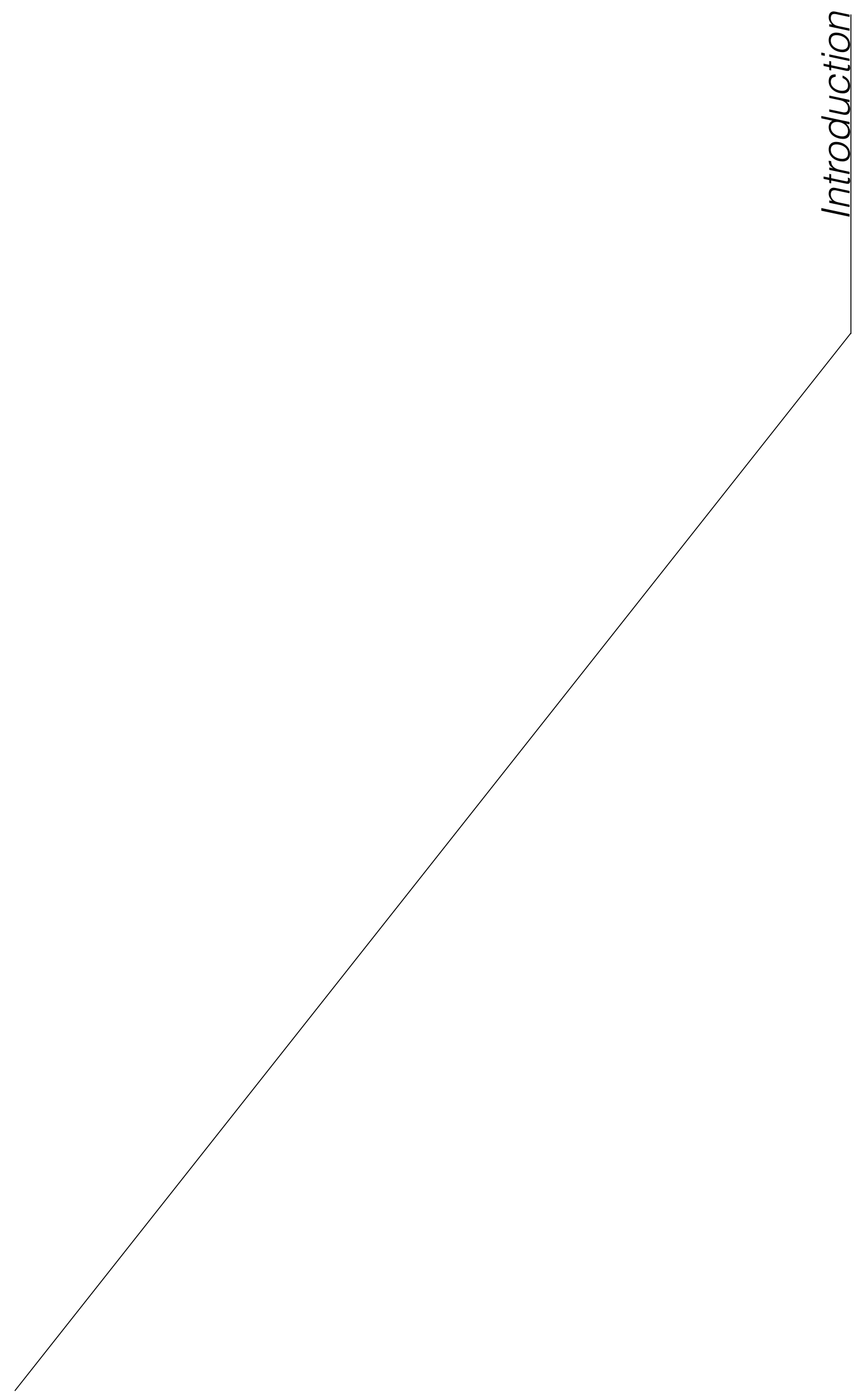




\section{Introduction}

\subsection{Alzheimer's Disease}

Alzheimer's disease is a devastating and the most common neurodegenerative disorder with currently 47 million people world wide suffering from the disease or a related form of dementia. Importantly, due to increased life expectancies this number is projected to increase to more than 131 million by 2050 (World Alzheimer Report, 2016) and does not take into account that currently only an estimated quarter of people suffering from $A D$ is being diagnosed. Besides the obvious burden this puts on family members of patients, the disease imposes a socioeconomic challenge to the entire population, as the total worldwide cost of dementia is estimated to become a trillion dollar by 2018. The most recent estimations by Hebert and colleagues sustained their report from 2003 in which they predict a two-fold increase of patients affected with AD in the next 20 years to 10.5 million in the USA alone (Hebert, Weuve et al. 2013).

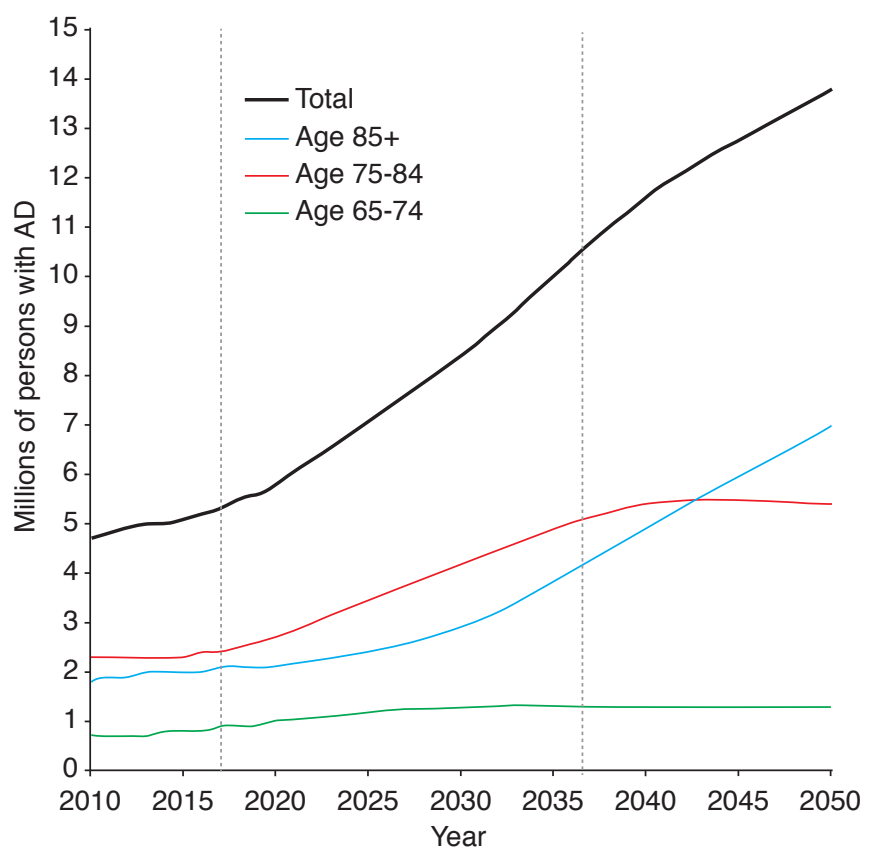

Figure 1.1: Alzheimer disease in the United States (2010-2050) estimated using the 2010 consensus.

Patients suffering from $A D$ will double in the next 20 years in the USA.Y-axis shows millions of persons affected with $A D, x$ axis shows years from 2010 to 2050. Especially age groups 85 (blue) and above and from 75 to 84 (red) will contribute to increased AD cases while 65-75 will not majorly contribute to the trend, due to the late onset of the disease. Dotted lines indicate current timepjoint and a doubling of patients affected by 2037. Illustration adapted from Hebert and colleagues. (Hebert, Weuve et al. 2013).

The disease is initially characterised by two principal phases. A first mild cognitive impairment $(\mathrm{MCl})$ phase which is symptomatically similar to age associated memory impairment (AAMI), and thus difficult to diagnose. And a second phase characterised by increasing decline of cognitive abilities such as perception, speech, and learning and memory. In the final stages, the patient is likely to loose speech, shows severe apathy and exhaustion, and is unable to execute simple tasks, and thus fully dependent on caregivers (Forstl and Kurz 1999). These symptoms are accompanied by different morphological characterisations, which are described post mortem e.g. according to Braak stage I-VI by the spreading of symptoms from initially the transentorhinal region (stage I-II), to the limbic regions (e.g., hippocampus, stage III-IV), finally to the neocortex (stage $\mathrm{V}-\mathrm{VI}$ ). Molecularly $\mathrm{AD}$ has been linked to protein aggregation, thus the two pathological hallmarks of $A D$ have been major targets in $A D$ research to treat causative 
factors. Firstly, amyloid precursor protein (APP) derived A $\beta$ plugs, and secondly neurofibrillary tangles (NFTs) composed of the microtubule associated protein tau. In rare familiar cases of the disease genetic mutations of these genes have been identified, which contributed to a basic understanding of the pathology. In contrast, while mostly displaying similar molecular pathologies, sporadic $A D$ it is likely multifactorial.

Several risk factor have been reported including, amongst others, genetic predispositions such as the apolipoprotein E (ع4) allele (APOE4) which was identified in genome wide association studies (GWAS) (Coon, Myers et al. 2007), head injury (Guo, Cupples et al. 2000), exposure to pollution (Calderon-Garciduenas, Reed et al. 2004), or nutrition (Luchsinger, Tang et al. 2002, Gustafson, Rothenberg et al. 2003). Further, twin studies report an influence of non-genetic risk factors in $A D$ and dementia. This influence contributes $21 \%$ to $42 \%$ to the appearance and progression of the disease, underlining the importance of the interplay between genetic risk factors and environmental circumstances (reviewed by Fischer 2014). However, an exact cause of the disease remains unknown. Despite intergovernmental agreement that $A D$ is a global public health priority (WDC, 2014/15) and establishing organisations such as G7-led "Global Action on Dementia", which encourages major efforts in research, there is to date no cure and no efficient treatment available to alter the course of the disease. Importantly, if a treatment for dementia would be identified, it will be crucial to make it easily available and provide high coverage. This puts four principal research strategies into focus namely modulating causative factors via antibody based therapies (Selkoe and Hardy 2016), target processing of causative factors by small molecules (Vassar, Kuhn et al. 2014), target causative factors directly by small molecules (Doig and Derreumaux 2015), and symptomatic treatments which are not targeting causative factors but instead circumvent pathological events (Fischer 2014, Benito, Urbanke et al. 2015).

\subsection{Molecular hallmarks of Alzheimer's Disease}

\subsubsection{Amyloid precursor protein and amyloid $\beta$}

In 1906 Alois Alzheimer dissected brain of patient Auguste Deter, the first case study of the disease, and described plaques of an unknown substance throughout the brain. It was until 1985 when Masters and colleagues biochemically purified and described amyloid $\beta(A \beta)$ for the first time (Masters, Simms et al. 1985). The genetic location of the precursor of $A \beta$ (amyloid precursor protein; APP) was localised on human chromosome 21 in 1987 (Goldgaber, Lerman et al. 1987, Kang, Lemaire et al. 1987), linking AD and trisomy-21 (Down-Syndrome), a genetic defect accompanied by cognitive impairment and $A \beta$ plaque depositions in the patients brains (Lott and Head 2005). Despite frequent studies and new links to neuronal processes, the physiological role of APP remains uncertain. The integral membrane protein is ubiquitously present including in the synapses of neurons, where it was reported to regulate synaptic formation, structure, and function (Priller, Bauer et al. 2006, Tyan, Shih et al. 2012). Further 
reports associated APP to changes in neural plasticity (Klevanski, Herrmann et al. 2015), acquisition of aversive memory (Priller, Bauer et al. 2006, Senechal, Kelly et al. 2008), cell adhesion, (Breen, Bruce et al. 1991), neuronal migration (Copenhaver and Ramaker 2016), neurite outgrowth (Milward, Papadopoulos et al. 1992, Qiu, Ferreira et al. 1995), and modulation of mitochondrial metabolism (Lopez Sanchez, Waugh et al. 2017). However, the underlying mechanisms not well defined and often disputed.

APP is processed by sequential cleavage of $\alpha-$ and $\beta$ - and secretase into two carboxyterminal fragments, which are in turn a substrate to the $y$-secretase processing them to either $p 3$ or $A \beta$, and the APP intracellular domain (Chow, Mattson et al. 2010). Of note, the A673T mutation in APP has been identified do have a preventive effects towards AD likely due to its proximity to the $\beta$ secretase cleavage site (Jonsson, Atwal et al. 2012, Kokawa, Ishihara et al. 2015). To initiate activity, $\mathrm{Y}$-secretase requires at least four components, namely presenilin (PS1 or PS2), nicastrin (Nct), presenilin enhancer 2 (Pen2), and anterior pharynx defective 1 (Aph-1) (Wolfe 2008). Cleavage to $A \beta$ occurs in the endosomal compartment and Golgi network, thus it believed that it is produced in those sub-cellular localisations and afterwards secreted through exocytosis. Of note, while reason remains unclear, also intracellular $A \beta$ was observed in human $A D$ and animal models (Wirths, Multhaup et al. 2001, Gomez-Ramos and Asuncion Moran 2007). Due to frequent cleaving events at alternative sites the length of $A \beta$ can range from 36 to 43 AA, while the predominant form is 40 AA long (Portelius, Brinkmalm et al. 2009, Mawuenyega, Kasten et al. 2013).

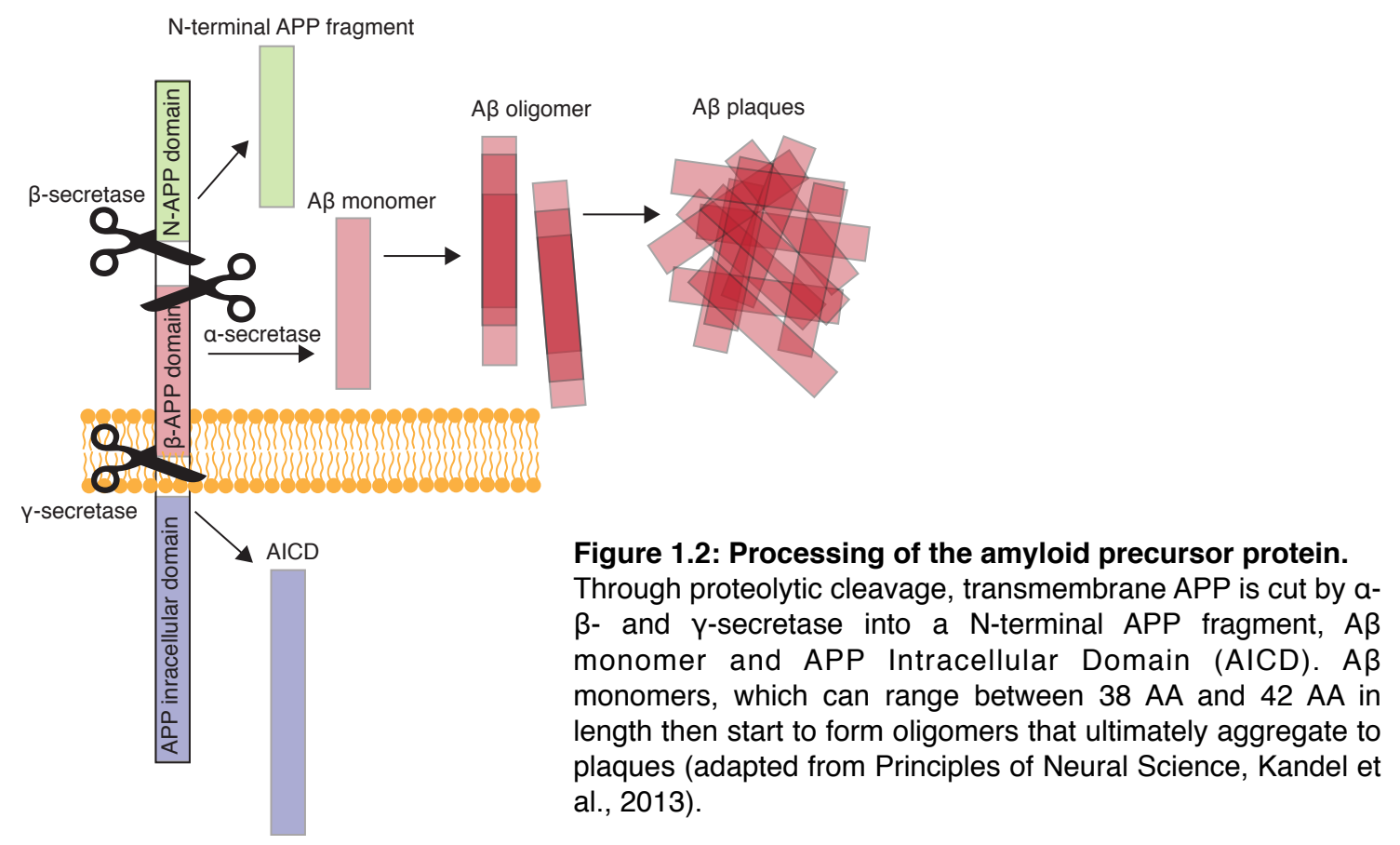

The conversion from non-toxic and soluble $A \beta$ into clumped toxic from, rich in $\beta$-sheets is considered a key event towards AD (Hardy and Selkoe 2002, Mattson 2004, Goate and Hardy 2012) and targeting amyloid pathology still represents a promising therapeutic approach (Haass and Selkoe 2007, Selkoe and Hardy 2016). Importantly, different A $\beta$ species show 
distinct amyloidogenic tendencies, while e.g. $A \beta 1-40$ or 1-38 are less and e.g. $A \beta 1-42, A \beta 3-$ 40 more prone to aggregate. As for APP itself the physiological function of $A \beta$ and pathological aggregation of its' distinct isomers is not well understood. Of the all possible species $A \beta_{1-40}$ and $A \beta_{1-42}$ are by far the most studied. While both species can form oligomers considered to be toxic, $A \beta 1-42$ is the main species found in early stage diffuse plaques, $A \beta 1-40$ strongly correlates to mature plaques (Iwatsubo, Odaka et al. 1994). Additionally, A $\beta 1-40$ oligomers were observed to damage the neuronal membrane (Canale, Seghezza et al. 2013) and was found to contribute to the formation of $A D$ (Shin, Ogino et al. 1997). Further, oligomeric non-plaque $A \beta$ aggregates are investigated as the primary pathogenic form of $A \beta$. These toxic oligomers, are thought to bind to surface receptors on neurons and alter the structure of the synapse or to form channels, thus disrupting neuronal communication. (Lacor, Buniel et al. 2007). One of the first explanations of neuronal dysfunction and toxicity mediated by $A \beta$ species is the channel hypothesis first proposed by Arispe and colleagues (Arispe, Rojas et al. 1993). It postulates that unregulated $A \beta$ ion channels result in a loss of ionic homeostasis, ultimately leading to neuronal dysfunction and cell death. Importantly, despite the fact that $A \beta$ deposition is pathologically increased in $A D$, neurotoxic and oligomeric species are not exclusive to $A D$, but can be detected in other diseases and cognitively healthy elderly (Price and Morris 1999), an observation underlining the importance to take additional molecular hallmarks of $A D$ into account. To be able to investigate the pathology efficiently good models are needed. One of the most applied models is the C57Bl6j-Tg(Thy1-APPsew;Thy1-PS1 L166P) mouse model for AD-like amyloidosis, herein APP/PS1. The model co-expresses KM670/671NL mutated APP and L166P mutated PS1 under the control of a neuron-specific Thy1 promoter element. The model shows several $A D$ hallmarks including cerebral amyloidosis starting at 6-8 weeks, a ratio of human amyloid which corresponds to $A D$ ( $A \beta 42$ to $A \beta 40$ of 1.5 in pre-pre-plaque and 5 in post-plaque, respectively). Further, the model shows extensive congophilic parenchymal amyloid and minimal amyloid angiopathy, as well as dystrophic synaptic boutons and hyperphosphorylated tau-positive neuritic structures. This makes APP/PS1 a well suited model to study therapeutic strategies and the pathological mechanism of amyloidosis.

\subsubsection{The microtubule associated protein Tau}

After the first characterisation of histopathological hallmarks in the brain of the first AD patient it took another 68 years until the successful isolation of neurofibrillary tangles (NFTs) from autopsied brains in 1974. Subsequently, their protein composition and identification of the major contributor, a protein of approximately $50 \mathrm{kDa}$, today known as the microtubule-associated protein tau was achieved (Iqbal, Wiśniewski et al. 1974, Weingarten, Lockwood et al. 1975). Even before that, NFT density was shown correlate with dementia and disease severity (Tomlinson, Blessed et al. 1970) and is today still the basis for classification of AD based on its' topography by Braak staging (Braak and Braak 1991). Findings from these and following studies strongly led to the tau pathology becoming a major area of research. Today 
entanglement of tau is generally considered to play a key role in the pathogenesis of Alzheimer's disease.

Tau is encoded by a single gene (MAPT) on human chromosome 17. In the human brain, the transcript is subject to alternative splicing which results in 6 isoforms. The translated proteins differ from one another in the number of amino-terminal inserts of 29 amino acids each, and the presence of three or four microtubule-binding domain repeats (Goedert, Spillantini et al. 1989), which have been linked to changes in microtubule affinity and altered disease progression (Goedert and Jakes 1990, Dickson, Kouri et al. 2011). Loss of the protein does not develop a detrimental phenotype in mouse models, possibly due to compensation by proteins of the same family (Harada, Oguchi et al. 1994). Tau was implicated in neurotransmission where it could act through the Fyn pathway (Roberson, Halabisky et al. 2011) and may serve in the nucleus in neuronal DNA and RNA protection (Violet, Delattre et al. 2014). In the healthy brain taus' main function is to promote assembly of tubulin subunits into microtubules and to stabilise the structure (Drubin and Kirschner 1986, Kadavath, Hofele et al. 2015). Importantly, this process is orchestrated by differential post-translational modifications of tau and particularly by its degree of phosphorylation, however, hyperphosphorylation suppresses this activity (Alonso, Zaidi et al. 1994) and can lead to tau aggregation under pathological conditions.

Post translational modifications (PTMs) of tau were first reported in 1992. Yasuo and colleagues identified several abnormally hyperphosphorylated sites on tau from the brains of individuals with AD, using mass spectrometry (Hasegawa, Morishima-Kawashima et al. 1992). Today 63 sites are known to be modifiable and 6 distinct PTMs were identified in models for human tau pathology (Morris, Knudsen et al. 2015). When tau becomes hyperphosphorylated, microtubule association is reduced and tau becomes prone to self-aggregation into paired helical filaments (PHFs) (Friedhoff, von Bergen et al. 1998), which manifest as neurofibrillary tangles (NFTs) in the neuronal soma, neuropil threads in neurites, and dystrophic neurites surrounding $A \beta$ plaques (Grundke-lqbal, lqbal et al. 1986). These hyperphosphorylated tau species show several-fold increased levels in AD patient brains, while the amount of non-hyperphosphorylated tau, however, is comparable between $A D$ brains and normal aged brains (Khatoon, Grundke-

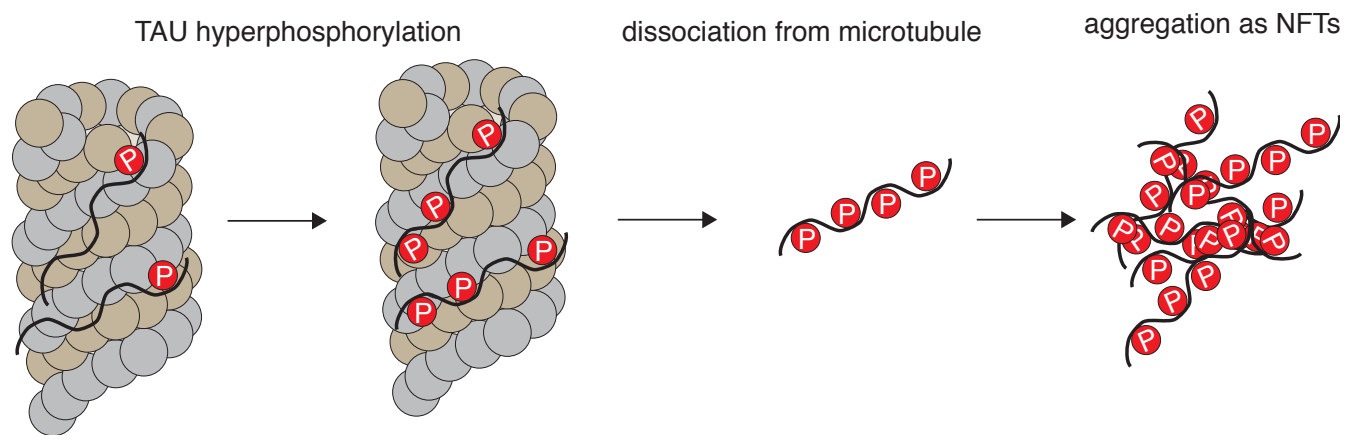

Figure 1.3: Aggregation of the microtubule associated protein tau.

Tau promotes assembly of microtubules and stabilises the structure. Hyperphosphorylation reduces affinity with microtubules and leads to aggregation, ultimately resulting in NFTs and cytotoxicity. 
lqbal et al. 1992, Kopke, Tung et al. 1993). In line with this, the protein phosphatase 2A (PP2A), which dephosphorylates hyperphosphorylated tau in healthy individuals was found to be compromised in brain tissue from individuals suffering from AD (Gong, Grundke-lqbal et al. 1994). However, it remains unclear which one of the several phosphorylation sites are essential for disease pathogenesis and which sites become phosphorylated after the formation of tau pathology. Additionally, It is not well understood which of the tau phosphorylation sites identified are essential for disease pathogenesis and which sites may become phosphorylated only after the formation of tau pathology in the tauopathies.

Further, there is significant evidence that mis-folded tau can propagate, exhibit prion like properties and to spread trans-synaptically (de Calignon, Polydoro et al. 2012, Liu, Drouet et al. 2012, Goedert, Masuda-Suzukake et al. 2017). This was demonstrated amongst others by studies showing that injection of brain extracts from tau transgenic mice or human tauopathy into the brains of mice expressing wild-type human tau, results in tau aggregation around the injection site and connected brain regions (Clavaguera, Bolmont et al. 2009, Ahmed, Van der Jeugd et al. 2014). Of note, several other PTMs of tau were identified, including ubiquitination (Cripps, Thomas et al. 2006), truncation (Zhang, Song et al. 2014), sumoylation (Dorval and Fraser 2006), and acetylation (Min, Cho et al. 2010, Min, Chen et al. 2015). Acetylation of tau has received little attention in the past but is emerging as an important PTM in its' physiological and pathological functions. Tau lysine (K) acetylation at residues 259, 290, 321 and 353 is decreased in AD but occurs in healthy human brain, and appears to protect tau from increased phosphorylation and suppresses tau aggregation (Cook, Carlomagno et al. 2014). Conversely, acetylation of tau at K 174, 274 and 280 was detected in AD brains, and may be pathological (Min, Chen et al. 2015). These findings suggest, that specificity is the key in the functionality of Tau acetylation. Sirtuin 1 (SIRT1) and the histone deacetylase 6 (HDAC6) were identified as the enzyme responsible for the deacetylation of tau and inhibition of HDAC6 enhanced tau acetylation and decreased tau phenotype in vivo (Cook, Carlomagno et al. 2014). This demonstrated that tau acetylation influences tau phosphorylation and aggregation directly, and suggests that tau acetylation could be a feasible target to ameliorate the pathology.

It is well established that tau hyperphosphorylation and other PTMs lead to aggregation and formation of NFT. This was repeatedly linked to neurotoxicity in AD (Lasagna-Reeves, CastilloCarranza et al. 2011, reviewed in Spires-Jones, Kopeikina et al. 2011), however to date there is no efficient treatment or cure available. Current approaches include immunotherapies using specific antibodies, pharmacological modulation of phosphorylation e.g. by PP2A enhancement (reviewed inVoronkov, Braithwaite et al. 2011), and symptomatic treatments without targeting causative factors (Fischer 2014, Benito, Urbanke et al. 2015).

In any of these approaches tau transgenic mouse models are valuable to investigate the role of tau protein in $A D$ and other tauopathies. However, common models often display motor dysfunction which is fatal for appropriate scoring in cognitive testing. An appropriate model for 
testing the influence of a treatment on cognition using behavioural tests is the mouse model C57BI6j-TG(THY1.2-Tau22) (herein Tau22) that expresses human 4-repeat tau mutated at sites G272V and P301S under control of the Thy1.2-promotor (Schindowski, Bretteville et al. 2006). This model does not show any motor dysfunction, while recapitulating key features of tau pathology such as hyperphosphorylation of tau on AD-relevant epitopes (AT8, AT100, AT180, AT270, 12E8, pSer396, and AP422), NFT like somatic inclusions and mild astrogliosis. The model is characterised by deficits in hippocampal synaptic transmission and impaired behaviour with increased anxiety, delayed learning from 3 months, and reduced spatial memory at 10 months of age. Taken together, this makes Tau22 a well suited model to study therapeutic strategies and the pathological mechanism of amyloidosis.

\subsection{Learning and Memory}

In higher animals the complex environment imposes the ability to learn and memorise events and features such as resources or threats as it is crucial for survival. Without this ability, future avoidance of harmful situations would be impossible and there would be no option to profit from previously discovered environmental factors, such as food resources. While, learning and memory are highly dependent on one another, both processes have to be treated as distinct (Bailey, Bartsch et al. 1996). The ability to acquire, consolidate, maintain and retrieve memory is far spread amongst metazoan and studied in different model organisms including $C$. elegans, $D$. melanogaster, and M. musculus. Historically the understanding of the fundamentals of learning and memory took a milestone in 1890 when William James stated in his 'Law of Neural Habit' that "when two elementary brain processes have been active together or in immediate succession, one of them, on reoccurring, tends to propagate its excitement into the other". The principle of contiguity derived from this initial revelation and states that the co-activity of

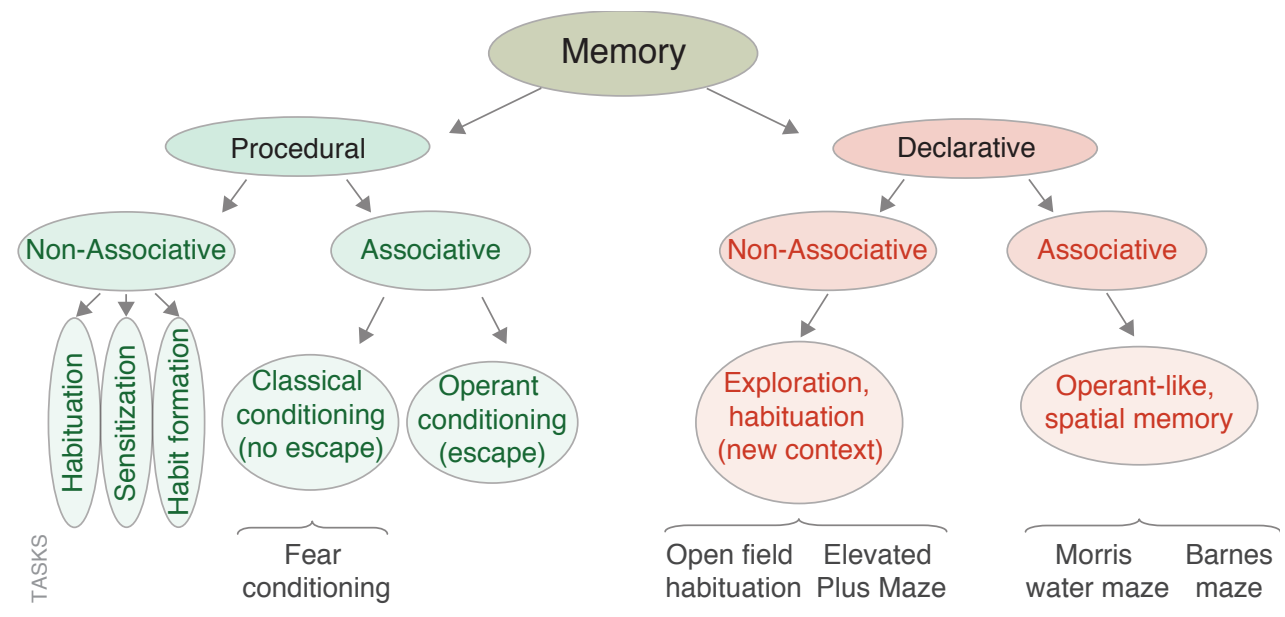

Figure 1.4: A possible memory taxonomy.

Different forms of memory and the most relevant cognitive tests associated for mouse models including contextual Fear conditioning, open field, elevated plus maze, Morris water maze and Barnes maze. Adapted from Quillfeldt 2006 
fundamental brain processes drives association. After Ramón y Cajál identified single neurons in the nervous system in 1911, the term "synapse" was designated as an interface for neuronal communication. Donald O. Hebb refined this statement in 1949 proposing that "when an axon of cell $A$ is near enough to excite a cell $B$ and repeatedly or persistently takes part in firing it, some growth process or metabolic change takes place in one or both cells such that A's efficiency, as one of the cells firing $B$, is increased".

In turn, the capability of neuronal circuits to adapt based on activity patterns is a crucial feature in learning and sustaining new information. In fact, the model of neuronal plasticity is essential in research investigating the cellular and molecular basis of understanding how memory is stored and retrieved. Although we do not fully understand the underpinnings of learning and memory, systematic approaches have been undertaken to gain insight into certain forms of learning and memory. Commonly memory is separated into "declarative" and "non-declarative" memory (Polster, Nadel et al. 1991, Dudai 2002). Non-declarative memory holds information e.g. about motor procedures and changes performance rather than conscious retrieval (Lum, Conti-Ramsden et al. 2012). In contrast, declarative memory is described as the memory used for facts and information we can retrieve and declare to others and further divided into associative and non-associative learning and their persistence. Importantly, both declarative and non-declarative memory include heavily hippocampus dependent forms of memory.

\subsubsection{The hippocampus in learning and memory}

Memory storage involves several brain areas which differ depending on the quality of the memory. The best studied example is the memory acquisition through integration of information in the hippocampus on short-term, which is then transferred to higher cortical regions such as the prefrontal cortex for permanent storage. Importantly, a recent paper by Kitamura and colleagues demonstrated that memory engram cells are formed in the prefrontal cortex and the hippocampus simultaneously and are later consolidated by an unknown mechanism (Kitamura, Ogawa et al. 2017). Thus, the hippocampus is a highly important brain area commonly recognised to be crucial for memory acquisition and consolidation, particularly in spatial memory. It is located in the medial temporal lobe in primates and below the cerebral cortex in other mammalians. In other vertebrates the important function of the hippocampus is fulfilled by

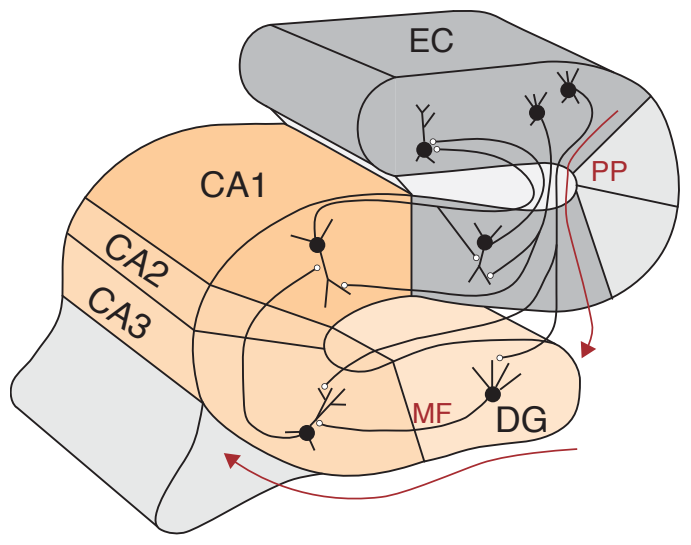

Figure 1.5: The Hippocampus.

Dentate gyrus (DG, bottom) and cornus ammonis sub regions (CA1, CA2, CA3) receive innervation via the perforant pathway (PP) from the entorhinal cortex (EC, top). Signalling within the hippocampus occurs mostly directed from DG through mossy fibres (MF) through CA areas to EC. Adapted from Kandel 2013 
evolutionary homologous but differently organised structures (Rodriguez, Lopez et al. 2002). The most important findings contributing to our understanding of hippocampal function stem from the case study of the patient known as "H.M.". Due to seizures he had his medial temporal lobe, including the hippocampus, surgically removed in 1957 and suffered from then on from severe anterograde amnesia of his declarative but not his procedural memory (Scoville and Milner 1957). The findings from this case study were complemented with several ablation experiments in non-human primates and rodents which underlined the hippocampal involvement in spatial learning (Moser, Moser et al. 1993), associative learning and recognition (Mahut, Zola-Morgan et al. 1982), and in the consolidation of fear memory (Sutherland, O'Brien et al. 2008). Structurally and functionally the hippocampus is further divided into dentate gyrus (DG) and cornus ammonis (CA), while the latter is subdivided into CA1, CA2, and CA3. The hippocampus signals to different brain regions from the $C A$, which in turn receives input from the DG via mossy fibers (Scharfman 2007) and receives input via the perforant path from the entorhinal cortex. Further, the hippocampus has been shown to connect to the amygdala (Saunders and Rosene 1988), the septum (Farr, Uezu et al. 1999), basal ganglia (Thierry, Gioanni et al. 2000), hyptothalamus (Cui, Gerfen et al. 2013), ventral tegmental area (Kahn and Shohamy 2013) prefrontal cortex (Thierry, Gioanni et al. 2000) and the cerebellum (Yu and Krook-Magnuson 2015). The dorsal and ventral regions of the hippocampus have been shown to serve different functions. While the dorsal hippocampus has been reported to be involved in spatial memory (Lee and Kesner 2003), the ventral hippocampus was linked to fear and stress response due to its closer association to the amygdala (Bast, Zhang et al. 2001). Notably, the hippocampus and more precisely the DG is one of the scarce sites of adult neurogenesis (Ming and Song 2005). Importantly, it has been shown that Inhibition of adult neurogenesis interferes with hippocampus-dependent memory function (Winocur, Wojtowicz et al. 2006).

Further, the hippocampus is highly relevant in $A D$, as the gradual loss of hippocampus dependent memory abilities is one of the first severe cognitive hallmarks of the disease. This is accompanied by the most striking molecular pathologies, namely $A \beta$ plaque deposition, NFT formation and neuronal loss, which occur first in the hippocampus and spread to higher brain areas (cf. Braak staging) (Dickson 1997, Serrano-Pozo, Frosch et al. 2011).

\subsubsection{Persistence of memory}

Memory storage can be divided into two principal categories depending on their persistence. Firstly, short-term memory (STM) which can hold very limited information and decays in order of seconds. Secondly, long-term memory (LTM), a much longer lived storage which can hold a large number of memories for hours do days and converges into the life time lasting persistent memory, which is resistant to disruption. The precise timely definition of memory stages is under debate (Cowan 2008), as the precise biological mechanisms are not trivial to identify. The temporary definition of STM is dependent on the field of research, as neurologists define it to 
last from seconds to a few minutes (Peterson and Peterson 1959), while cellular neurobiologists consider STM last for up to a few hours (Goelet, Castellucci et al. 1986).

STM is characterised by its particularly quick access with limited capacity. A precise measurement of the latter is difficult, due to individual variation between subjects in how many information items or "chunks" can be stored as this process is dependent on the chunk quality and can be distorted by rehearsal (Cowan 2008). However, an average value of around seven items temporarily sorted in STM is estimated with some variance (Miller 1956). Hypothetically, a memory inflicting event would occur in a specific brain region, such as the hippocampus, and result in a specific excitation pattern ("engram"), which represents the memory trace and decays over time (Mongillo, Barak et al. 2008). Of note, this proposed passive mode of decay is still very controversial and within engram research an actively studied topic (Lewandowsky, Duncan et al. 2004, Kitamura, Ogawa et al. 2017).

Conversely, LTM is characterised by virtually unlimited storage capacity, however, with longer access latency (Bailey and Kandel 2008). As for STM the precise timing defining memory as LTM is debated, as the initial phase of memory storage, "consolidation" is highly variable (Bailey, Bartsch et al. 1996). For example, cellular consolidation takes place within a few minutes after an event (Schafe, Nader et al. 2001), while system consolidation can take up to weeks and longer (Dudai 1996). Further, different brain areas, memory qualities, and species show considerably large variations in the consolidation process (Shadmehr and Brashers-Krug 1997). The remarkable theoretical capacity of LTM was impressively demonstrated by the case study of $\mathrm{AJ}$, the first case study of hyperthymestic syndrome in which episodic memory is pronounced. AJ was capable of recalling precisely what she did on a given date and what week day it fell on for her entire life (Parker, Cahill et al. 2006).

The underlying mechanism of both STM and LTM are still of current interest in neurodegenerative disorders since an impairment can be observed in a variety of diseases including post-traumatic stress disorder (PTSD) (Landré, Destrieux et al. 2012), age associated memory impairment (AAMI) (Bender, Naveh-Benjamin et al. 2010, Chen and Naveh-Benjamin 2012), Multiple sclerosis (Thornton and Raz 1997), Schizophrenia (Landgraf, Steingen et al. 2011), and AD (MacDuffie, Atkins et al. 2012)

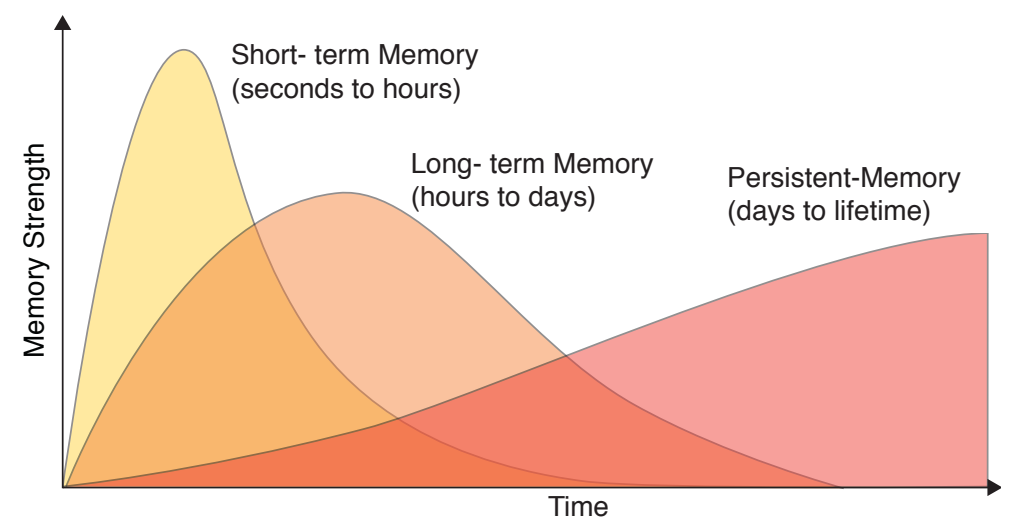

Figure 1.6: Time dependent stages of memory.

Short-term memory (STM) has limited storage $( \pm 7$ chunks) and decays within seconds to hours. Long-term memory (LTM) with large storage for memories and lasts for hours do days and converges into persistent memory, which is resistant to disruption and lasts for a lifetime. (adapted from Dudai 2002) 


\subsubsection{Association of memory}

In this form of memory an association between two stimuli or between stimulus and response is learned. Associative learning is the most common form of memory acquisition in everyday life, including amongst others learning of a foreign language, playing an instrument, or fear of painful situations. Further, it is divided into operant conditioning and classical or pavlovian conditioning. The latter was first described by Nobel Prize laureate Ivan P. Pavlov in 1927 (re-published Pavlov 2010). In his studies on digestion, Pavlov described salivation in dogs upon the sight of the empty food dish. This observation led him to design an experimental paradigm, in which a neutral stimulus, the ringing of a bell, was followed by the offering of meat powder to the test subjects. The test subjects associated the ringing of the bell or "conditioned stimulus" (CS) with the access to meat powder or "unconditioned stimulus" (US). After several repetitions of the conditioning, salivation was evoked in the animals by the presentation of the CS ("ringing bell"), without application of the US ("food"), thus a conditioned response (CR) was learned (republished Pavlov 2010). Today, several animal behavioural tests are variations of pavlovian learning including the common contextual fear conditioning (Mcllwain, Merriweather et al. 2001, Rudy, Huff et al. 2004), an important tests in hippocampus dependent memory research.

Conversely, in operant conditioning, the outcome of the experiment depends only on the subject's behaviour, which itself triggers the associative stimulus. Burrhus F. Skinner, coined the term with his invention of the operant conditioning chamber, while Edward L. Thorndike conducted the first experiments in 1898. In Skinners experiments rats were subjected into a chamber in which it was allowed to explore freely. Further the chamber contained a lever, which the rat would most likely hit by accident, rewarding the animals with food. After several accidental repetitions the number of events in which the lever was pressed increased until the rat had no more desire to eat. Notably, the Skinner box still has diverse applications. Operant conditioning was adapted to different spatial navigation tasks to test memory in operant-like spatial learning paradigms. Most relevant today are the Morris water maze, a pool with several visual cues to find a submerged invisible escape platform (Morris 1981) and the Barnes maze a circular plate with 4 visual cues and 20 holes, one of which harbours an escape cage, specifically designed to detect memory deficits associated with senescence (Barnes 1979).

In contrast, non-associative learning is solely dependent on a single quality stimulus, instead of establishing a connection between at least two stimuli of different quality and quantity. Nonassociative learning can be separated into sensitization, habituation, and conversely dishabituation. In sensitization the response to a given stimulus is enhanced above the baseline trough the presentation of a strong stimulus. Frequent repetition of the presentation of a stimulus identical in quality and quantity leads to a reduced reaction, considered as habituation. In contrast, dishabituation, restores the response to a formally habituated stimulus, usually by applying another, often strong, stimulus. In 1987 Eric Kandel and colleagues established these mechanisms in the neural system responsible for the gill withdrawal reflex in the sea slug Aplysia californica, and further demonstrated that these mechanisms were due to alterations in 
the synapses between sensory and motor neuron. Interestingly, they also showed that the decrease of the gill withdrawal due to repeated stimulation during short-term was based on decreased transmitter release. Conversely, in long-term habituation, which is an effect present after repeated stimulation for days, the altered stimulus response was due to a reduction of synaptic connections between the two neurons involved. Besides demonstrating nonassociative learning Kandel and colleagues revealed the concept of a basic cellular mechanism of both short- and long-term memory effects in invertebrates.

\subsubsection{Molecular mechanisms of learning}

Memory storage on a cellular level has been of great interest in neuroscience and it is believed that the formation and strengthening of neuronal connections trough synapses, so called synaptic plasticity, is a cellular correlate of learning and memory. Thereby, synaptic strength is in principle dependent on frequency and repetition of a stimulus as stated in the Hebbian postulate.

During STM the cellular and molecular changes occurring in memory forming neurons are thought to be based on the covalent modifications of pre-existing proteins by a broad spectrum of different kinases (Goelet, Castellucci et al. 1986, Bailey and Kandel 2008), conversely modifications required for LTM formation are more extensive and requite de-novo protein synthesis. Neurons change their structure, form new synapses, and grow dendritic spines after training. To enable such changes, synthesis of structural molecules, including proteins, is necessary. This was demonstrated in 1962 by Flexner et al. (Flexner, Flexner et al. 1962), when they applied the protein synthesis inhibitor (PSI) puromycin to mice upon which they showed deficits to learn and retain memory, remarkably intracerebral injections of PSI resulted in LTM deficits even 1 day past training, stressing the importance of de novo protein synthesis. These results were subsequently supported with different PSIs and various model organisms (Agranoff and Klinger 1964, Barondes and Cohen 1966, Gold 2008). Importantly, the disruption of LTM by protein synthesis inhibition did not affect STM (Flood, Rosenzweig et al. 1973), which was only abolished after application of $\mathrm{Ca}^{2+}$ influx interference agents, targeting the synaptic NMDA receptor (Gibbs and Ng 1977).

In search for a model to study cellular learning in vertebrates Bliss and Lømo discovered in 1973 that isolated brain sections of the rabbit hippocampus respond differently dependent on electrical stimulation (Bliss and Lomo 1973). They discovered that neuronal responses remained stable when stimulated with a mild, low frequent electric pulse, while tetanic stimulation caused excessive firing of neurons and resulted in an increased excitatory postsynaptic potential (EPSP), which remained elevated over time. The increase of synaptic response after tetanic stimulation was termed long-term potentiation (LTP). Conversely, synaptic efficiency was reduced upon a long patterned stimulus, a phenomenon described as long-term depression (LTD). Of note, while LTP and LTD resemble physiological learning events in associativity, durability, and the need for de novo protein synthesis (for late phase LTP) (Frey 
and Morris 1998), it remains uncertain to which extend they are a cellular counterpart to learning and memory (Bliss and Collingridge 1993).

Formation of LTM in response to a stimulus requires de-novo protein synthesis an in turn differential gene expression. This mechanism is best investigated in the induction of LTP and the two necessary receptors glutamate receptors, namely NMDA-type and AMPA-type (Dale and Kandel 1993, Trudeau and Castellucci 1993). The simultaneous activation of the two receptor types results in calcium influx. In turn, this starts an enzymatic cascade ultimately resulting in short-term effects at the post-synapse by an increase of AMPA-R and increase of available transmitter, mediated by Adenylate cyclase (AC). If the stimulation is prolonged, longterm synaptic alterations are initiated. The increased activity of $A C$ results in high levels of cyclic adenosine monophosphate (CAMP) and in turn acts as a second messenger and activates the protein kinase $A(P K A)$, which together with the mitogen-activated protein kinase (MAPK) relocates to the nucleus and promotes transcription through phosphorylation of transcription factors (TFs). (Bacskai, Hochner et al. 1993, Martin, Casadio et al. 1997, Kandel 2001). The initiation of transcription is a tightly controlled process involving the interaction of chromatin and various proteins, including TFs. They are highly conserved in evolution, while some are essential for the basic process of transcription, others orchestrate the selective repression and activation of certain genes. TFs are regulated temporally e.g. in development and circadian clock or upon an external stimulus. They then control transcription indirectly or bind specific DNA motifs directly. A well studied example is the TATA binding protein (TBP), which binds to a DNA consensus sequence characterised by repeating $\mathrm{T}$ and $\mathrm{A}$ in the promoter region of a gene, thereby regulating RNA synthesis (White and Jackson 1992, Watson 2014). Together with TBP associated factors, TBP constitutes the polymerase II pre-initiation complex, thus enhances the transcription of the respective gene sequence (Lee and Young 2000). However, regulation of gene expression is a complex process orchestrated by several basal and regulated TFs, particularly in memory formation activation protein 1 (AP1), early growth response factor (Egr), CCAAT enhancer binding protein (C/EBP) and the cAMP response element binding protein (CREB) were demonstrated to play important roles.

The latter (CREB) is a particularly well studied factor in learning and memory. CREB binds the CAMP response element (CRE), which is contained within the control region of a gene and is activated by CAMP. The binding of TFs to CRE was shown to determine the expression level of certain genes by regulating RNA polymerase activity. Binding of CRE through different TFs was shown to regulate RNA polymerase activity and thereby determines the expression level of a given gene (Montminy, Low et al. 1986, Montminy and Bilezikjian 1987). Most of the findings stem from implicit memory in invertebrates, however a similar yet more complex signalling cascade that leads to the activation of CREB has also been observed in the mammalian brain (Barco, Alarcon et al. 2002, Lonze and Ginty 2002, Pittenger, Huang et al. 2002). However, the precise mechanism is to date still unresolved, though the importance of CREB in learning and memory was underlined by experiments, which showed CREB binding interference abolished 
LTP and LTM formation (Dash, Hochner et al. 1990, Pittenger, Huang et al. 2002, reviewed by Kandel, Dudai et al. 2014). In summary this pathway is an excellent demonstration how an external stimulus is first conveyed to the transcriptional machinery and subsequently translated into gene expression changes. It demonstrates that synaptic plasticity in LTM formation is dependent on transcriptional plasticity ultimately leading to protein synthesis. Importantly, in research on memory, cognitive abilities, and impairments in particular this puts systems into focus which are able to orchestrate the crosstalk between environmental input and regulation of gene expression. This is further supported by the observation of de-regulated gene expression in brain diseases linked to memory impairment including AD (Blalock, Buechel et al. 2011, Kim, Moon et al. 2012, Mills, Nalpathamkalam et al. 2013). In line with this, epigenetics and chromatin remodelling in particular gained large interest in the past decades to understand transcriptional regulation. The importance of histone acetylation in learning was first demonstrated in a mouse model, where mice showed impaired memory consolidation after the activity of the CBP/KAT3A was reduced (Alarcon, Malleret et al. 2004, Korzus, Rosenfeld et al. 2004). Afterwards, it was repeatedly demonstrated that alteration in the chromatin state modulated LTP, LTM related synaptic plasticity, and spatial memory (Guan, Giustetto et al. 2002, Fischer, Sananbenesi et al. 2007, Guan, Haggarty et al. 2009, Lubin, Gupta et al. 2011, Benito, Urbanke et al. 2015, Kosarussavadi, Pennington et al. 2017).

\subsection{Epigenetics}

The sequencing the entire human genome in the late 20th century (Lander, Linton et al. 2001, Venter, Adams et al. 2001) held promising potential to identify inheritable diseases and formerly unknown genetic features. However, the genetic information is not complete without an index orchestrating the transcriptional machinery. As early as 1957 Conrad Waddington published in his "strategy of genes" a hypothesis explaining how cells with identical genetic background could develop into distinct cell types and form different tissues and is thus the founder of the epigenome (epi = Greek: $\varepsilon \pi i$-over, above, outer). This is today thought to not only orchestrate, but extend genetic information beyond DNA base pairing and be able to interact with the environment and translate external input into genomic instructions. Under certain circumstances the epigenetic code be transmitted the progeny and is involved in the differentiation from cell to organism (Jenuwein and Allis 2001). In humans the DNA was estimated to contain between 19,000 and 25,000 protein coding genes (International Human Genome Sequencing 2004, Ezkurdia, Juan et al. 2014), and a total of $3 \times 10^{9}$ base pairs (bp) including non-coding sequences (Vogel 1964). Stretched out this would be around 2 meters of DNA which needs to fit into a single nucleus (Molfese 2011). To achieve this compression the genetic information is packed into nucleosomes, which consist of $147 \mathrm{bp}$ of DNA wrapped around a histone octamer containing each of the four core histones twice (Woodcock and Dimitrov 2001), an accessibility trade-off to achieve a 10,000-fold condensation. Chromatin is not uniform, but varies in its' 


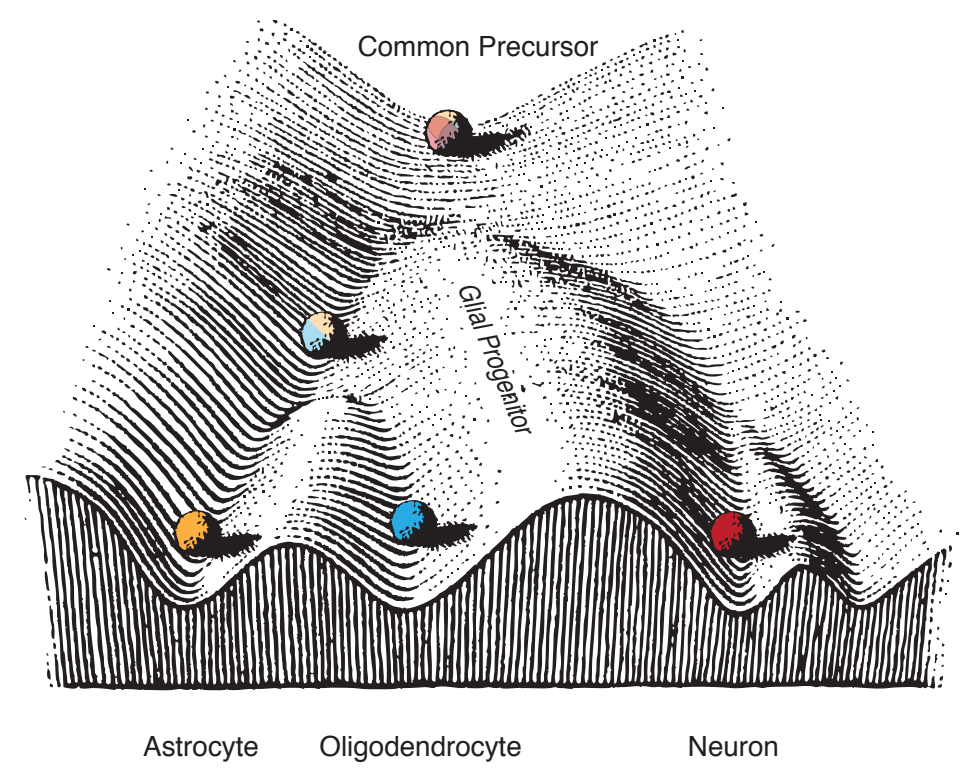

Figure 1.7: The epigenetic landscape.

The identical genetic information is illustrated as a marble, cell fate as valleys. Depending on epigenetic orchestration (e.g. DNA methylation) the same genetic information gives rise to e.g. Astrocytes, Oligodendrocytes or Neurons. Adapted from Waddington, 1957

packaging from the mostly inactive, highly condensed heterochromatin to the transcriptionally active and less condensed euchromatin. Most eukaryotic genes are transcribed by RNA polymerase II and in vitro experiments have shown that Pol II cannot traverse through nucleosomes (Chang and Luse 1997). These spatial restrictions are overcome by ATPdependent nucleosome remodellers with orchestration of transcriptional processes by the epigenetic machinery (Das and Tyler 2013).

The three main areas of epigenetic modifications are Non-coding RNA species, DNA methylation, and integration of non-canonical histones/post-translational modifications of histone termini ("histone tails"). Non-coding RNAs constitute the majority of the transcriptome, the best studied subgroup are micro RNAs (miRNAs), which are 19-22 nucleotides in length and catalyse gene silencing by binding to mRNA (Im and Kenny 2012). DNA methylation is conveyed via DNA methyl-transferases (DNMTs) by addition of methyl groups to $\mathrm{CpG}$ islands, which is generally thought to mediate transcriptional repression (Miller, Campbell et al. 2008), while demethylation is poorly understood. Thereby, the transcriptional state of certain areas can be altered through altered chromatin-structure (Allis, Jenuwein et al. 2007). Further, the charged and flexible histone tails are modifiable at several residues by several PTMs.

\subsubsection{Chromatin plasticity}

Early studies on bulk histone acetylation demonstrated that high acetylation levels correlate with higher accessibility to interacting proteins (Hebbes, Clayton et al. 1994, Krajewski and Becker 1998). Further, it was observed that the ground state of chromatin, independent of transcriptional activity, shows intermediate levels of $\mathrm{H} 3$ and $\mathrm{H} 4$ acetylation, due to a mix of untargeted HAT and HDAC activities (Vogelauer, Wu et al. 2000) thus wide acetylation of histone 
$\mathrm{H} 3$ and $\mathrm{H} 4$ leads to less condensed chromatin, which does not tightly correlate with active transcription per se, but rather indicates area of transcriptional capability. Interestingly, in the rodent hippocampus $\mathrm{H} 3$ and $\mathrm{H} 4$ acetylation is differently regulated after fear conditioning. Here, H3 acetylation was elevated after exposure to a novel environment paired with a foot shock, while $\mathrm{H} 4$ acetylation was increased when the pre-exposure was extended (Levenson, O'Riordan et al. 2004). Of note, this demonstrated that learning induces measurable changes in hippocampal histone acetylation levels, which was confirmed in subsequent studies by pharmacologically elevating acetylation levels (Fischer, Sananbenesi et al. 2007, FontanLozano, Romero-Granados et al. 2008, Koshibu, Graff et al. 2009). However the precise mechanisms and functional consequences of most acetylation events and recruited complexes remain largely unknown (Filippakopoulos and Knapp 2012). The histone octamer is constituted of $\mathrm{H} 2 \mathrm{~A}, \mathrm{H} 2 \mathrm{~B}, \mathrm{H} 3, \mathrm{H} 4$ and the histone linker $\mathrm{H} 1$ or their non-canonical substitutes (Kamakaka and Biggins 2005). The structure of the small 11 to $22 \mathrm{kDa}$ proteins is among the most evolutionary conserved (Kornberg and Lorch 1999) and contains a high amount of lysine and arginine. The basic structure of the four core histones is similar consisting of a central folddomain, amino- and carboxy-terminal (tails). While the fold-domain contributes to the histone octamer, the tails are involved in regulatory cellular processes and chromatin structure (Morales and Richard-Foy 2000). A broad array of PTMs, which often occur simultaneously, has been identified including methylation (Taverna, Li et al. 2007), ubiquitinylation (Davie and Murphy 1990), glycosylation (Sakabe, Wang et al. 2010), sumoylation (Shiio and Eisenman 2003), phosphorylation (Stucki, Clapperton et al. 2005), and acetylation (reviewed in Rousseaux and Khochbin 2015), each signalling to the chromatin polymer in their specific way (Cheung, Allis et al. 2000). The by far best studied modifications of histone tails are the $\mathrm{N}$-terminal methylation and acetylation (reviewed in Bannister and Kouzarides 2011, Molfese 2011). The addition and removal of these moieties is mediated by the counteracting activities of enzymes, so-called writers and erasers. Such modifications directly affect the interaction of histones with the DNA and thus alter the chromatin state (Bannister and Kouzarides 2011). Biochemical studies on histones of the central nervous system showed incorporation of [14C]-acetate into the histones of different rat brain regions during a learning experiment. A well studied example of the mediation of the necessary changes is histone acetylation, by histone-acetyltransferases (HAT, writer) and histone-deacetylases (HDAC, eraser) (Johnson and Turner 1999, reviewed in Fischer, Sananbenesi et al. 2010). The key mediators of these changes, HATs and HDACs, are both highly specialised enzyme families that (de)acetylate specific amino residues of the target histone tail. Thus both HATs and HDACs are a diverse family. While the former are not well studied, the latter are dependent on the cofactors $\mathrm{NAD}^{+}$or $\mathrm{Zn}^{2+}$ and comprise 18 members in human and are divided into 4 classes based on their homology to yeast HDACs (de Ruijter, van Gennip et al. 2003, Seto and Yoshida 2014). The Class I consists of Rpd3-like proteins (HDAC1, HDAC2, HDAC3, HDAC8), Class II of Hda1-like proteins (HDAC4, HDAC5, HDAC6, HDAC7, HDAC9, HDAC10), Class III Sir2-like proteins (SIRT1, SIRT2, SIRT3, SIRT4, SIRT5, 


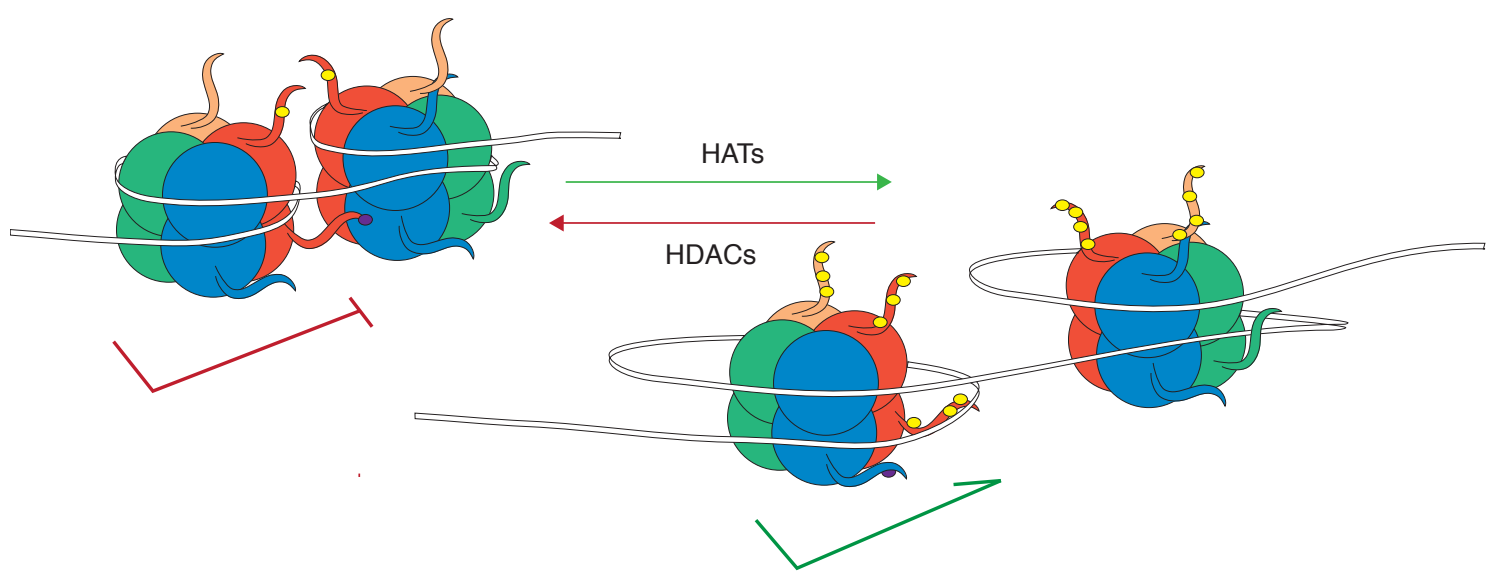

Figure 1.8: The counteracting function of writers and easers.

Writers (e.g. HATs) add PTMs to histone tails thereby increasing accessibility for the transcriptional machinery, thus transcription is promoted. Conversely, erasers (e.g. HDACs) remove modifications, which leads to more compact state, here the transcriptional machinery is hindered, thus gene expression is reduced. Note that at baseline some modifications are present in more condensed state.

SIRT6, SIRT7), and Class IV protein (HDAC11). In neuroepigenetics particularly Class I and Class II HDACS have gained large interest du to their cognition modulation capabilities (reviewed in Fischer 2014).

\subsubsection{HDACs in learning and memory}

Class I HDACs are located in the nucleus and range from $22 \mathrm{kDa}$ to $55 \mathrm{kDa}$ in size with great homology of the catalytic sites. Every member of the group has been investigated and linked to cognitive function with similar results, suggesting a general role as molecular inhibitors of memory formation. For example, HDAC2, the best studied member of the class, was found to be expressed throughout the brain by several neuronal cell types and oligodendrocytes but not astro-and microglia (Yao, Zhang et al. 2013). Further, HDAC2 was shown to impair contextual fear learning and spatial memory formation when overexpressed and to improve memory function when deleted in neurons of mice (Guan, Haggarty et al. 2009). This was sustained by structural findings showing that loss of HDAC2 increased the number of synapses (Guan, Haggarty et al. 2009). Importantly, another study showed that enhanced learning behaviour in HDAC2 deficient mice correlated with increased hippocampal acetylation of Histone 4 at lysine 12 (H4K12) acetylation. This mark was repeatedly demonstrated to play a key role in memory reinstatement in the ageing hippocampus through alternative splicing (Peleg, Sananbenesi et al. 2010, Benito, Urbanke et al. 2015).

Class II HDACs are larger compared to Class I, ranging between $120 \mathrm{kDa}$ to $135 \mathrm{kDa}$ and feature a nuclear export signal (NES) as well as a nuclear localization signal (NLS), which is in line with their shuttling function. Class II HDACs are further divided into Class Ila (HDAC4, HDAC5, HDAC7, HDAC9) and Class Ilb (HDAC6, HDAC10) based on their homology, as Class Ilb contains a second catalytic domain not present in other HDACs. 


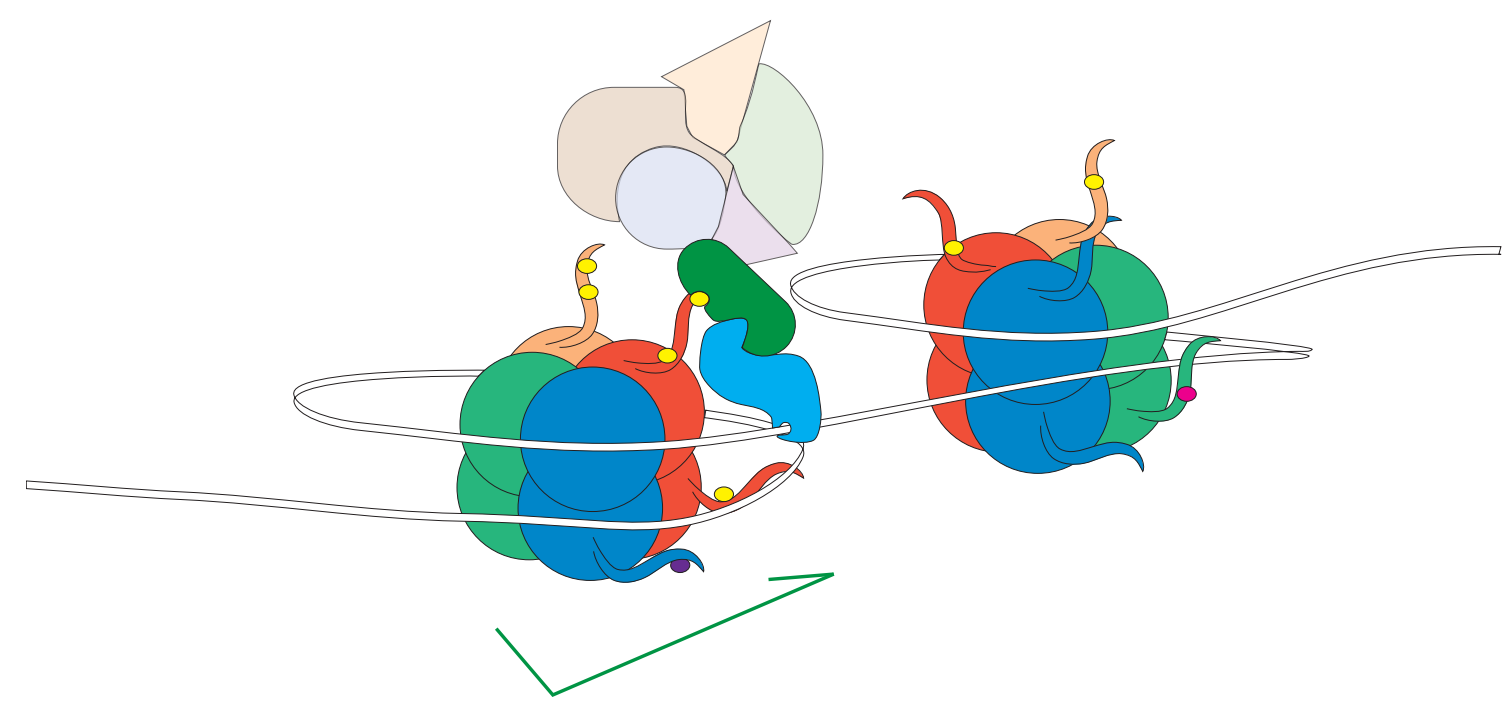

Figure 1.9: Chromatin readers read the histone code.

Readers of the histone code recognise specific moieties at histone $\mathrm{N}$-terminals. Upon recognition readers serve as scaffold and recruit further factors such as HATs, HDACs, or TFs to the site and initiate differential outcomes, e.g. structural changes in chromatin and/or transcriptional changes.

HDAC6 is outstanding in its class since the main targets are cytoplasmatic proteins such as tubulin or heat-shock protein 90 (Zhang, Li et al. 2003, Ding, Dolan et al. 2008). Further, loss of HDAC6 in mice does not show a phenotype and does not influence cognitive function, including memory (Govindarajan, Rao et al. 2013). Importantly, HDAC6 was shown to also deacetylate the microtubule associated protein tau (Zhang, Li et al. 2003, Ding, Dolan et al. 2008, Noack, Leyk et al. 2014). Since HDAC6 levels positively correlate with tau burden (Cook, Gendron et al. 2012) this process was linked to tau clearance. Additionally, the authors demonstrated that HDAC6-mediated loss of Tau acetylation makes the protein susceptible to pathological phosphorylation (Cook, Gendron et al. 2012). In a drosophila model HDAC6 reduction was shown to rescue hyper-phosphorylated Tau induced microtubule instability and neurodegeneration (Xiong, Zhao et al. 2013). In line with this, it could be shown that HDAC6 deficiency rescued memory function in the APP/PS1 model for AD (Govindarajan, Rao et al. 2013).

In summary, these are well studied examples of how the addition and removal of chemical moieties to the highly flexible histone tails is orchestrated. However, there is a gap in the current understanding of how chromatin structure which is seemingly littered with PTMs is translated into precise biological outcomes or extends the informational content. This cannot only be explained by steric interaction between histone tails and DNA. The combinatorial nature of these modifications are believed to give rise to the histone code, which is recognised by proteins so-called chromatin readers that serve as scaffold to recruit complexes that activate or repress transcription (Strahl and Allis 2000). 


\subsubsection{The Readers of the Histone Code}

The histone code hypothesis stems from the function of three protein classes. Writers and erasers, which add and remove histone tail modifications and the so-called readers, which recognise the wide variety of modification patterns at the histone tails and scaffold for various transcriptional regulators (Strahl and Allis 2000). In addition to steric interaction between DNA and histones, which is most relevant in core histone modifications (Cosgrove, Boeke et al. 2004), the combinatorial pattern recognition of chromatin readers provides a fine-tuned mechanism to regulate chromatin dynamics and in turn selective transcriptional changes. Complexes associating with specific marks are often composed of multiple readers that each can feature several recognition sequences. This allows for coordinated binding so several PTMs and may provide a lock and key mechanism to target specific genomic regions and generate precise biological responses. It is this precise mode of histone PTM recognition that is the foundation of the histone code hypothesis (Jenuwein and Allis 2001).

The first chromatin reader was discovered in 1999 as the bromodomain containing PCAF, which was found to bind acetylated lysine (Dhalluin, Carlson et al. 1999). Since then a wide variety of readers interacting with different motifs including those previously mentioned were identified (Tan, Luo et al. 2011). However, the best studied members are readers of methylation and acetylation (Yun, Wu et al. 2011). Of the readers identified the heterogenous family of bromodomain containing proteins are currently in the focus of research as they have been shown to modulate transcription (Bartholomeeusen, Xiang et al. 2012) and demonstrate promising drug targets in cancer (Qiu, Jackson et al. 2015, da Motta, Ledaki et al. 2017) and cognition (Korb, Herre et al. 2015, Sartor, Powell et al. 2015, Benito, Ramachandran et al. 2017). The bromodomain is comprises around 110 amino acids and selectively recognises acetylated lysine residues. This conserved domain was found in transcriptional regulators and chromatin-remodelling proteins, among others HATs such as PCAF and TAF1, but also members of the BET family of nuclear proteins (BRD2, BRD3, BRD4, and BRDT) (Zeng and Zhou 2002). Particularly the latter contribute significantly to the understanding of how chromatin changes are translated into specific genomic commands. For example, it was shown that BRDT binds $\mathrm{H} 4$ that is diacetylated at K5 and K8 (Moriniere, Rousseaux et al. 2009), while BRD2 specifically recognises the acetylation of H4K12ac (Kanno, Kanno et al. 2004, Umehara, Nakamura et al. 2010). Particularly, the recognition of H4K12ac is of great interest to elucidate the role of readers in learning and memory, since deregulation in learning-induced hippocampal H4K12 acetylation prevented hippocampal gene expression associated with memory formation correlated with significantly impaired memory in mice (Peleg, Sananbenesi et al. 2010). H4K12 acetylation was also demonstrated to be associated with spatial and fear learning (Bousiges, Neidl et al. 2013). Further, acetylation of H4K12 is associated with differential transcription of immediate early genes and memory consolidation (Bousiges, Vasconcelos et al. 2010, Bousiges, Neidl et al. 2013) a mechanism which is repressed by APP (Hendrickx, Pierrot et al. 2014). Elevated acetylation of H4K12 was also observed in human patients with $\mathrm{MCl}$ (Plagg, 
Ehrlich et al. 2015). The notion that H4K12ac and readers thereof are key factors in learning and memory was underlined by our finding that $\mathrm{H} 4 \mathrm{~K} 12 \mathrm{ac}$ is reduced around the transcription start site (TSS) in models for AAMI and AD. Further, decreased acetylation of the site correlates with memory impairment, while reinstating acetylation correlates with improved spatial learning and reinstated transcriptional activity (Benito, Urbanke et al. 2015). The acetylation of $\mathrm{H} 4$ was linked to alternative exon usage mediated by alteration of the elongation rate. An increase of acetylation facilitated RNA polymerase II (RNAPol-II) and exon exclusion in 3 different instances (Schor and Kornblihtt 2009, Hnilicova, Hozeifi et al. 2011, Zhou, Hinman et al. 2011), a mechanism that was linked to the precise reinstatement of acetylation at H4K12 in 2 models for cognitive decline (Benito, Urbanke et al. 2015). This underlines not only the importance of readers of this mark but indicates that chromatin modifications and readers thereof modulate learning in memory, possibly in a splicing dependent manner. Importantly, it was shown for Brd4 that it is recruited to $\mathrm{H} 4$ upon a extracellular signal where it distinguishes unactetylated $\mathrm{H} 4$ from acetylated at K5 and K12 (Dey, Chitsaz et al. 2003), where it regulated splicing during a heat shock (Hussong, Kaehler et al. 2017)

\subsubsection{On chromatin assisted alternative splicing}

Two models were proposed by Zhou a colleagues explain the influence of chromatin marks on alternative splicing events (Zhou, Luo et al. 2014). Firstly, "kinetic coupling" essentially assumes a competition between transcriptional elongation and the splicing machinery, since alternative exons require more time to be recognised and thus are skipped when elongation speed increases. Secondly, the "chromatin splicing adaptor system" explains the same phenomenon by chromatin associating proteins that recognise and bind specific histone marks and direct splicing factors to the site of transcription, in turn resulting in two outcomes A) core components of the spliceosome are recruited to remove introns B) splicing regulators are recruited through chromatin remodelling proteins to directly affect the inclusion or exclusion of alternative exons. These models could be envisioned to synergistically contribute to alternative exon usage in a scenario in which a chromatin reader scaffolds at its respective mark and recruits elongation factors and spliceosome components to TSS. Through cluster density of multiple initiation and elongation complexes elongation speed would be regulated. Strong representation would result in slowed down elongation leading to exon inclusion along the gene body, while a cleaner chromatin structure with few scaffolds would increase elongation speed resulting in exon skipping.

\subsection{Annexins}

This family of $\mathrm{Ca}^{2+}$ dependent proteins was recently linked to chromatin remodelling, trough its member annexin A2 (Oh, Gao et al. 2013). The annexins are a large family of proteins consisting of more than 160 members and are present in all higher eukaryotic cell types, 
indicating fundamental cellular roles (Gerke and Moss 2002). Out of the family, 12 different members were identified in mammals, namely A1-A11 and A13 (Gerke and Moss 2002, Moss and Morgan 2004). All mammalian annexins show similar key functions. The first function identified was the affinity towards membrane bound phospholipids in presence of $\mathrm{Ca}^{2+}$. Further, interactions with various proteins, in particular with members of the $\$ 100$ family were identified (Rintala-Dempsey, Rezvanpour et al. 2008). Following the initial findings, several binding partners and cellular roles of the annexins were identified including extracellular receptor activity, membrane trafficking, membrane-cytoskeleton interactions, signal transduction, and RNA binding. Generally annexins are today considered to be a family of multifactorial proteins (Gerke and Moss 2002, Gerke, Creutz et al. 2005, Hitchcock, Katz et al. 2014, Lauritzen, Boye et al. 2015). While all annexins show cytosolic localisation, some members were shown to translocate or reside with a fraction inside the nucleus, including annexin A11 and A2, (Eberhard, Karns et al. 2001, Tomas and Moss 2003).

Annexin A2 (AnxA2) is a well studied member of the Annexin family. Like all annexins, it has a highly conserved core domain with four homologous repeats of 70-80 amino acids (AAs), so called annexin repeats, and a mild curvature with a concave and a convex side. The core domain stretches from AA 31 to AA 338 with known binding sites for $\mathrm{Ca}^{2+}$, heparin, actin and phospholipids, which are homologue to other annexins (Drucker, Pejic et al. 2013). The 30 AA long $\mathrm{N}$-terminus (tail) contributes significantly to separate functions of AnxA2 from other annexins. The tail is subject to complex regulation by PTMs that are in turn modifying its cellular functions. AnxA2 is a direct substrate of SRC kinase and Ser11, Ser25 and Tyr23 comprise important phosphorylation sites to control sub-cellular localisation (Grindheim, Saraste et al. 2017). Additionally, the tail region is crucial for protein-protein interaction, especially for $S 100 /$ AnxA2 complex formation (Eberhard, Karns et al. 2001, Nazmi, Ozorowski et al. 2012, Liu, Myrvang et al. 2015). Indeed, AnxA2 shows two main configurations either as 36-kD monomer or as a heterotetramer in which two monomers bind to a pair S100A10 (p11) (Waisman 1995). Moreover, AnxA2 binds multiple other intra- and extracellular ligands to convey different cellular responses (Vedeler, Hollas et al. 2012). AnxA2 is broadly studied in cancer where it was linked to tumour progression (Wang and Lin 2014). However, it remains to be elucidated whether AnxA2 is protective or contributes metastasis.

Besides showing distinct pools in the cytosol and nucleus (Vishwanatha, Jindal et al. 1992) AnxA2 was demonstrated to shuttle between the compartments. While the precise mechanism is not fully understood, the distribution of Annexin A2 seems to be controlled by the release from the AnxA2/p11 heterotetramer initiated by tail phosphorylation and conveyed via the nuclear export signal (NES) located from AA 3 to AA 12 (Eberhard, Karns et al. 2001). Shuttling of AnxA2 was observed from the membrane to the nucleus (Liu and Vishwanatha 2007). And involvement of AnxA2 in cellular processes was reported from ECM to nucleus, suggesting further translocations (Raynal and Pollard 1994, Deora, Kreitzer et al. 2004, Zheng, Foley et al. 2011, Xiu, Liu et al. 2016, Aukrust, Rosenberg et al. 2017). For example, a change in 
localisation was observed in response to increased genotoxic stress in ageing (Wei and Lee 2002, Strosznajder, Jesko et al. 2005, Lin and Beal 2006).

In the cytosol AnxA2 was reported to mediate the aggregation and fusion of liposomes in a $\mathrm{Ca}^{2+}$ dependent manner by binding to negatively charged phospholipids (Blackwood and Ernst 1990, Liu, Fisher et al. 1995). This mechanism contributed to the findings of a study in cancer, where the authors showed that high extracellular levels of AnxA2 correlate with metastasis, a poor prognosis, and neoangiogenesis (Mussunoor and Murray 2008). Another study suggest this effect could be complemented by exosomal AnxA2 which promotes angiogenesis in breast cancer (Maji, Chaudhary et al. 2017). A finding that is in line with a report in an in vivo mouse model for AnxA2 deficiency, where the authors showed defects in neovascularization and fibrin homeostasis (Ling, Jacovina et al. 2004). The membrane bound function is complemented with the function of AnxA2 at the extracellular matrix (ECM). Here, AnxA2 was shown to be involved in ECM degradation and ECM remodelling through the activation of serine proteases (Sharma and Sharma 2007). Importantly, modulation of the ECM has been proven to bidirectionally modulate memory formation, and to promote synaptic plasticity and memory extinction (Dityatev and Schachner 2003, Senkov, Andjus et al. 2014, Tsilibary, Tzinia et al. 2014).

In contrast, nuclear AnxA2 was linked to RNA binding and transcription in multiple occasions. Several studies found AnxA2 to be present in the nucleus and to associate to the nuclear matrix (Arrigo, Darlix et al. 1983, Vishwanatha, Jindal et al. 1992, Eberhard, Karns et al. 2001, Grindheim, Hollas et al. 2016). Already in early studies AnxA2 was implicated with DNA replication, based on the interaction with the DNA-polymerase a (Jindal, Chaney et al. 1991). A finding which was complemented with another report demonstrating a direct binding of AnxA2 to DNA in vitro (Boyko, Mudrak et al. 1994) and to recognise and potentially recruit primers (Kumble, Iversen et al. 1992, Vishwanatha, Jindal et al. 1992). More recent studies linked AnxA2 either directly to transcriptional regulation or placed it upstream of cascades ultimately leading to altered gene expression. For example, it was linked to a kinase cascade regulating the angiotensin 2 induced transcription of cyclooxygenase 2 (Derbyshire, Halfter et al. 2005). Another instance where AnxA2 was found upstream of a signalling cascade was reported in the increase of NF-B and catenin after progastrin stimulation (Sarkar, Swiercz et al. 2011). Further, it was reported that AnxA2 is involved with the transcriptional regulation of its direct interaction partner S100A10, plasmin, and fascin in L5178Y cells (Hou, Yang et al. 2008). Additionally, AnxA2 was reported toact upstream of the signal transducer and activator of transcription 6 (STAT6), promoted its nuclear entry and in turn activates transcription (Das, Shetty et al. 2010). Interestingly, AnxA2 was found to be accumulated at the proximal Col1A2 region, putting it into close association with the transcriptional machinery, where it could facilitate the binding to the produced mRNA, thus controlling collagen protein synthesis (Schafer, Hitchcock et al. 2015). This is in line with previous findings implicating AnxA2 as a RNA binding protein, which is possibly also involved in RNA processing (Vedeler and Hollas 2000, Filipenko, MacLeod et al. 2004, Mickleburgh, Burtle et al. 2005, Hollas, Aukrust et al. 2006, Aukrust, Hollas et al. 2007). 
This was further supported by Filipenko and colleagues who suggested that AnxA2 either increases c-myc RNA stability and its transport to polysomes or is an activator of its translation (Filipenko, MacLeod et al. 2004). Additionally, AnxA2 was reported to be a cellular RNA binding protein, modulating the frameshifting efficiency of viral RNA, which suggests an anti-viral function, and implies AnxA2 in RNA metabolism for other cellular mRNA (Kwak, Park et al. 2011).

While reports on AnxA2 focus largely on cancer research, little is known about its roles in the brain. Wang and colleagues demonstrated that AnxA2 and SNAP-23 interact in a $\mathrm{Ca}^{2+}$ dependent manner (Wang, Chintagari et al. 2007). Interestingly, SNAP-23 is enriched in dendritic spines where it co-localizes with components of the postsynaptic density and modulates NMDA receptor currents (Suh, Terashima et al. 2010). In depression treatment with selective serotonin re-uptake inhibitors, AnxA2 was shown to associate to the SMARCA3 chromatin remodelling complex in neurons, and to orchestrate action of the complex possibly through its actin binding capabilities (Oh, Gao et al. 2013). Additionally, AnxA2 was shown to change its location during spatial learning in neurons (Zhao, Waisman et al. 2004) and is associated with frontotemporal dementia (FTD) (Gauthier-Kemper, Weissmann et al. 2011). In induced astrocytes of FTD patients AnxA2 was found to be upregulated and to interact with Tau (Hallmann, Arauzo-Bravo et al. 2017).

In summary, AnxA2 is a multifactorial protein with specific actions from the extracellular space all the way into the nucleus and possibly transmits signals not only by initiation of cascades but by shuttling. In the nucleus AnxA2 has repeatedly been implicated with the transcriptional machinery, RNA binding, and moreover with the SMARCA3 chromatin remodelling complex, placing it into close context with the epigenetic machinery. 
$\frac{0}{0}$ 


\section{Objectives}

Alzheimer's Disease (AD) is detrimental neurological disorder that arises on the background of amyloid plaques, neurofibrillary tangles and neuronal cell death as the major hallmarks. Due to increased life expectancies the number of patients affected with $A D$ is predicted to increase dramatically in the next decades. However, to date there is no cure and no efficient treatment available to alleviate the burden $A D$ imposes on our society. The onset and progression of sporadic $A D$ are believed to critically depend on combinations of genetic and environmental risk factors. Such genome-environment interactions are mediated via the epigenetic machinery. There is increasing evidence that epigenetics plays a key role in neurodegenerative diseases and offers novel therapeutic strategies for neuroprotection and regeneration.

The motivation of my thesis work was therefore (1) to investigate the epigenetic processes in cognitive function in health and diseases; (2) to study the therapeutic potential of novel compounds with a specific focus on efficacy in an advanced stage of the diseases: and (3) to develop a neuronal cell culture system that would allow the analysis of transcriptome plasticity after manipulation in a single-cell specific manner. More specifically the three pillars of my thesis work were:

1. Identify proteins that interact with the learning-associated epigenetic mark H4K12ac and their involvement in cognitive function and potential as new therapeutic targets agains $A D$

Age-associated memory impairment is tightly linked to acetylation of H4K12. Thus, my aim was to identify chromatin readers of this mark and to test whether they are potential drug targets for a symptomatic treatment of $A D$.

\section{Find novel drug- and genetic-based targets for symptomatic treatment of $A D$}

a) Test the efficacy of anle138b to reinstate physiological cellular function and in vivo cognitive performance

Amyloid pathology is believed to be causative for the onset of AD. However, strategies targeted towards the clearance of amyloid plaques have so far failed in translational research. As part of my thesis work I tested the novel compound anle138b in AD-like amyloidosis, which was reported to be beneficial in other aggregopathies.

\section{b) Test the efficacy of deleting HDAC6 to ameliorate AD-associated symptoms}

Previous data of the lab suggested that inhibition of histone-deacetylase 6 (HDAC6) ameliorates memory impairment in a mouse model for amyloid deposition. In addition, to amyloid burden, Tau-pathology is a key hallmark of AD. I therefore generated Tau mutant mice that lack HDAC6 and characterised their cognitive phenotypes 
3. Develop a technology to study neuronal network dynamics at the subpopulation and individual cellular level

A high-risk high-gain aim of my thesis was to develop a neuronal cell culture system that would allow us to manipulate selected cells in a neuronal network and subsequently analyse transcriptome plasticity separately in cells directly and in-directly affected by disease-associated cells. The rational behind this approach were data from other cellular systems (e.g. immune cells) in which only few cells need to undergo transcriptional changes in order to affect (either in a beneficial or detrimental manner) the entire cell population. I envisioned that such as system for neuronal networks would allow us to address the dynamics of various disease processes 


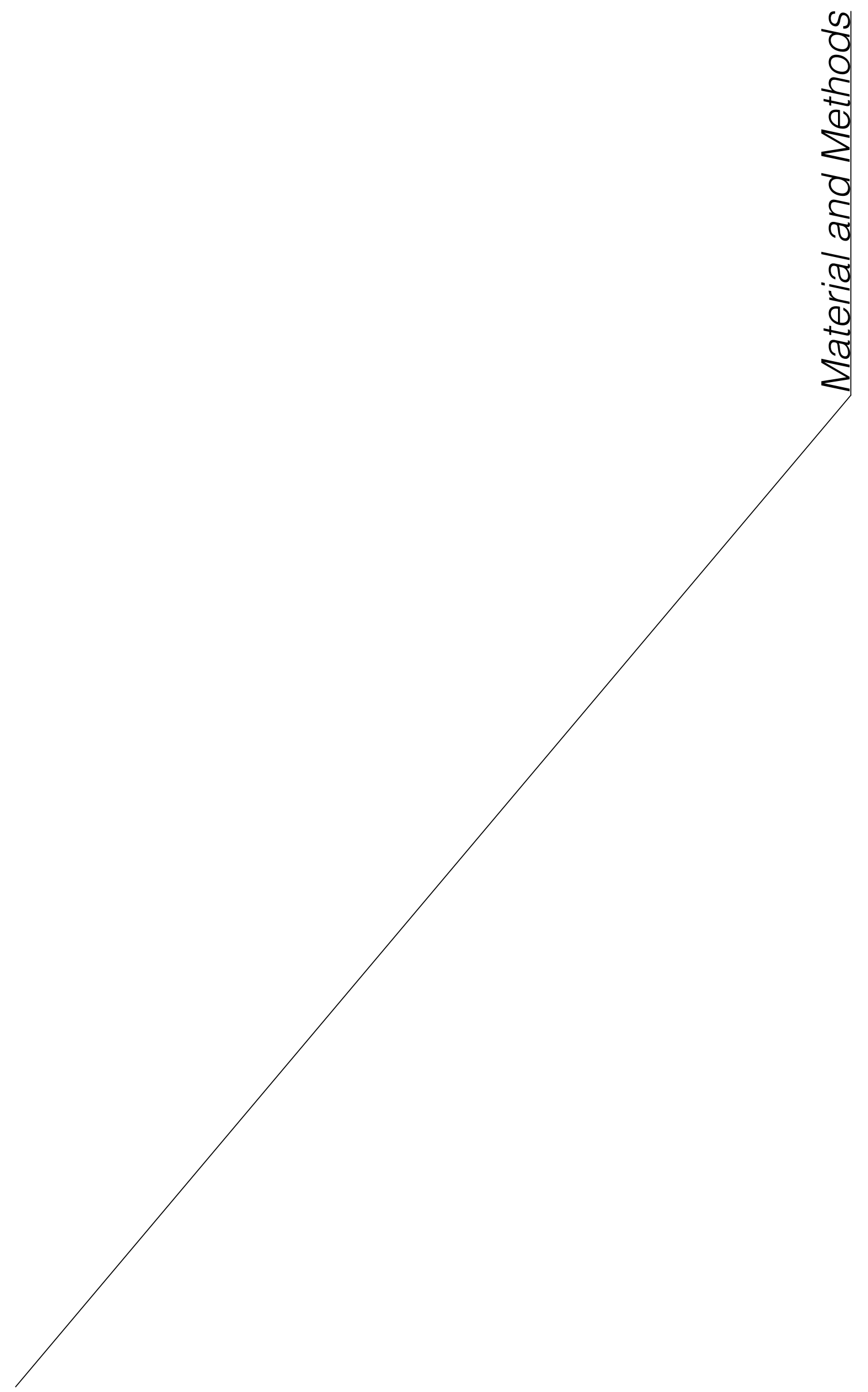




\section{Material and Methods}

\subsection{Animals}

\subsubsection{Animal welfare}

All animals in context of this thesis were bred and housed under standard Laboratory conditions with both food and water supplied ad libitum and a $12 \mathrm{~h}$ light cycle. Animals were bred in-house and kept for ageing in groups of up to 5 animals. Before behavioural experiments animals were relocated to a separate behaviour room and single caged to avoid hostile behaviour after individual tests. Animals were allowed to habituate to the new environment for 1-2 weeks prior to behavioural testing. If not stated otherwise only male mice were used in behavioural experiments. Depending on experiments animals for cell culture were bred in house (AnxA2-/) or delivered from Janvier (CD1-SWISS, E15). Pregnant animals were housed solitarily or in groups of 2 in case of Janvier delivered animals.

\subsubsection{Thy1.2-Tau22 x HDAC6--transgenic mice}

Tg(Thy1-MAPT)22Schd (THY-Tau22) mice were a gift from Luc Buee and originally described by Schindowski and colleagues (Schindowski, Bretteville et al. 2006). HDAC6+- (Govindarajan, Rao et al. 2013) mice on 129 background were first backcrossed to C57BL/6JRj for 10 generations to generate a largely identical genetic background compared to THY-Tau22. The resulting strain was bred by crossing male THY-Tau22+ $+\pi$ to female HDAC6 ${ }^{-1+}$. Male mice of the genotype TAU+/+, Hdac6+/y (control); TAUT/+, Hdac/y (HDAC6 KO); TAU+/T, Hdac-ly (TAU / HDAC6 KO) or TAU $+/ T, \mathrm{Hdac}^{+/ y}$ (Tau) were aged for 6 month or 13 month of age to asses early stage and late stage pathology respectively (Fig. 3.1).
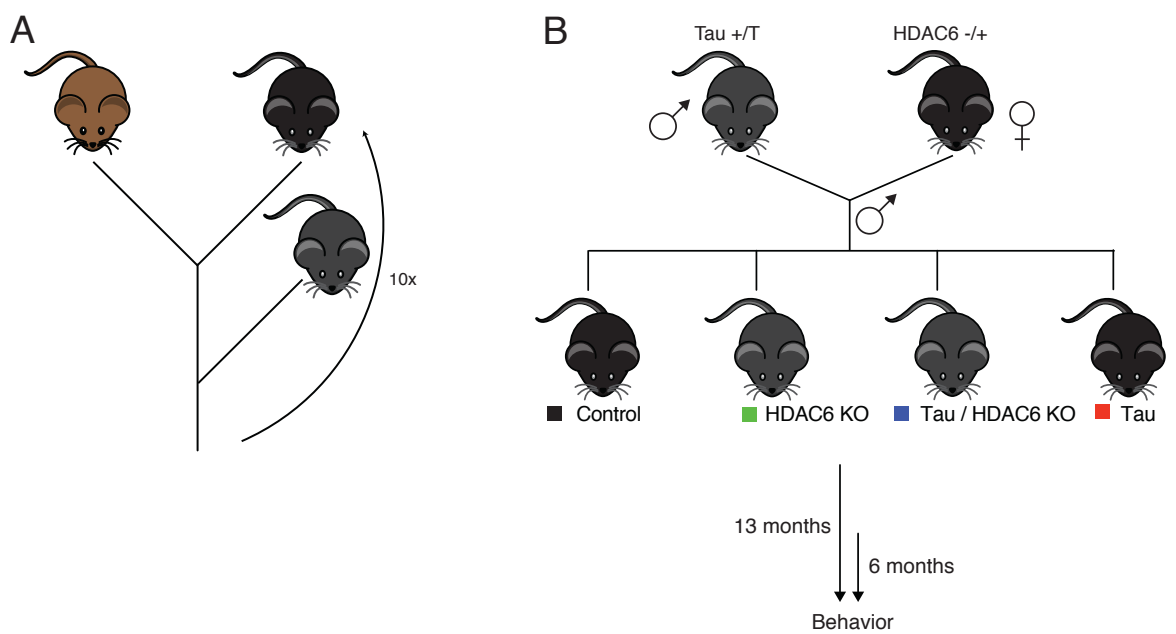

Figure 3.1: Generation of genetic background with double transgene.

Maternal generation on 129 Background was backcrossed for 10 generations to C57BL6/j (A). Paternal THY-Tau22+/T were mated to maternal HDAC6 ${ }^{-/+}$resulting in either Tau ${ }^{+/+}$, HDAC6 ${ }^{+/ y}$ (control); Tau /+,

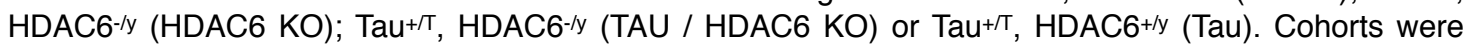
then aged to either 6 or 12 month $(B)$. 


\subsubsection{AnxA2 null mutant mice}

Animals for behavioural tests at 6 month of age were a kind gift of Manuela Schmidt and were originally generated by exchanging econ 3 and 4 by a NEO cassette (Ling, Jacovina et al. 2004). Animals were tested at 6 month of age and until then housed as described beforehand. For KO cultures and behaviour at 13 month Female AnxA2 null mutants who did not undergo behaviour were crossed to genetic background (C57BL/6JRj) in house to generate heterozygous progeny. AnxA2+/- where then mated to generate homozygous knockouts and wild-type littermates for behavioural experiments at 13 month of age.

\subsubsection{Genotyping}

To identify animal genotypes mouse tail biopsies were digested in $200 \mu \mathrm{l}$ DirectPCR tail-lysis buffer (Viagen Biotech) with $40 \mu \mathrm{g}$ Proteinase K (Roth) in a TermoMixer comfort (Eppendorf) at $1400 \mathrm{rpm}, 55^{\circ} \mathrm{C}$ for $2 \mathrm{~h}$ and inactivated at $85^{\circ} \mathrm{C}$ for $45 \mathrm{~min}$. Samples were then incubated at $4{ }^{\circ} \mathrm{C}$ for $1 \mathrm{~h}$ to allow residual debris to precipitate. Subsequently, PCR master mix composed of $2.5 \mu \mathrm{l}$ Dream Tag buffer, $2 \mu \mathrm{l}$ dNTP, $0.125 \mu \mathrm{l}$ DreamTaq, $18.375 \mu \mathrm{l} \mathrm{H} 2 \mathrm{O}, 0.5 \mu \mathrm{l}$ primer (each forward and reverse, $10 \mu \mathrm{M}$ ) was prepared and supplemented with $1 \mu \mathrm{l}$ Template. Primers were supplemented according to mouse line for either HDAC6, NEO-casette, Tau22, or AnxA2 (Table 3.1) and Cycler settings according to table 3.2-3.5. Electrophoresis on $1.5 \%$ agarose gel containing $0.06 \mu \mathrm{l} / \mathrm{ml}$ Midori Green (Takara CloneTech) was then used to visualise amplicons and results documented using the FAS V Gel Documentation System (Nippon genetics).

Table 3.1: Primers used for cloning, genotyping and pPCR

\begin{tabular}{|l|l|}
\hline Name & Sequence \\
\hline AnxA2_perfectmatch_F & CAAGGGAGGCTCTCAGCGATAC \\
\hline AnxA2_perfectmatch_R & CTGTGCTGAGCCCTTCAGTCA \\
\hline AnxA2_Nhe1_F & TAGCTAGCCAAGGGAGGCTCTCAGCGATAC \\
\hline AnxA2_BamH1_R & ATGGATCCCTGTGCTGAGCCCTTCCGTCA \\
\hline F_AnxA2_exon3_genotype & TGCCTACGGGTCAGTCAAAC \\
\hline R_AnxA2_exon3_genotype & GCAGGGATACAAAGCACCCT \\
\hline Thy-Tau22_F_genotype & ATGGCTGAGCCCCGCCAGGAG \\
\hline Thy-Tau22_R_genotype & TGGAGGTTCACCAGAGCTGGG \\
\hline HDAC6_F_genotype & GCCAGGGATTGGGTTAACACAAGCTAG \\
\hline HDAC6_R_genotype & CACACGCACGCACACCAGATAAACAC \\
\hline NEO_F_genotype & AGGATCTCCTGTCATCTCACCTTGCTCCTG \\
\hline NEO_R_genotype & AAGAACTCGTCAAGAAGGCGATAGAAGGCG \\
\hline AnxA2_Mali7_sybr_F & AGAGAAGGACCAAAAAGGAGC \\
\hline AnxA2_Mali7_sybr_R & CATACTGGGCAGGTGTCTTC \\
\hline
\end{tabular}


Table 3.2: Genotyping PCR Protocol Tau22

\begin{tabular}{|c|c|c|}
\hline Step & Temp. $\left({ }^{\circ} \mathbf{C}\right)$ & Time (min) \\
\hline Denaturing & 95 & 3 \\
\hline $\begin{array}{c}\text { Amplification } \\
(\times 30)\end{array}$ & 58.7 & 1 \\
\hline Elongation & 72 & 1 \\
\hline
\end{tabular}

Table 3.4: Genotyping PCR NEO casette

\begin{tabular}{|c|c|c|}
\hline Step & Temp. $\left({ }^{\circ} \mathbf{C}\right)$ & Time (min) \\
\hline Denaturing & 95 & 3 \\
\hline $\begin{array}{c}\text { Amplification } \\
(\times 35)\end{array}$ & 95 & 1 \\
\cline { 2 - 3 } & 72 & 1 \\
\hline Elongation & 72 & 10 \\
\hline
\end{tabular}

Table 3.3: Genotyping PCR HDAC6

\begin{tabular}{|c|c|c|}
\hline Step & Temp. $\left({ }^{\circ} \mathbf{C}\right)$ & Time (min) \\
\hline Denaturing & 95 & 3 \\
\hline $\begin{array}{c}\text { Amplification } \\
(\times 35)\end{array}$ & 95 & 1 \\
\hline & 72 & 1 \\
\hline Elongation & 72 & 10 \\
\hline
\end{tabular}

Table 3.5: Genotyping PCR AnxA2

\begin{tabular}{|c|c|c|}
\hline Step & Temp. $\left({ }^{\circ} \mathbf{C}\right)$ & Time \\
\hline Denaturing & 95 & $2 \mathrm{~min}$ \\
\hline $\begin{array}{c}\text { Amplification } \\
(\times 30)\end{array}$ & 58 & $30 \mathrm{~s}$ \\
\hline Elongation & 72 & $30 \mathrm{~s}$ \\
\hline & 72 & $10 \mathrm{~s}$ \\
\hline
\end{tabular}

\subsubsection{Intracranial Injections}

To inject adeno-associated viral particles (AAV) into the dentate gyrus (DG) of 3 month old male mice, animals were anesthetized (Ketamin $20 \mu \mathrm{l}$, Xylarem $16 \mu \mathrm{l}$, NaCl $0.9 \% 164 \mu \mathrm{l} ; 100 \mu \mathrm{l} / 10 \mathrm{~g}$ ) and subsequently treated with analgesic (Buprenorphine $30 \mu \mathrm{l}, \mathrm{NaCl} 0.9 \% 470 \mu \mathrm{l} ; 50 \mu \mathrm{l} / \mathrm{animal}$ ). Animals were then fixated in a 3-point stereotaxic setup and cranial skin was removed in a longitudinal incision to expose the skull. Holes for injection were drilled bilateral symmetrically relative to bregma (mediolateral: $+/-1 \mathrm{~mm}$; anteroposterior: $-1.75 \mathrm{~mm}$ ). For injection glass capillaries were prepared by filling with mineral oil to reduce gas compression during injection and washed with $2100 \mathrm{nl}$ PBS before virus uptake. $2000 \mathrm{nl}$ of virus sample were loaded and capillaries at $0.01 \mathrm{~mm} / \mathrm{s}$ introduced $2 \mathrm{~mm}$ on the dorsoventral axis into brain tissue until DG was reached. The tissue was given $3 \mathrm{~min}$ to fully invaginate the capillary before injection of $900 \mathrm{nl}$ virus solution at $250 \mathrm{nl} / \mathrm{min}$. Afterwards, the solution was given $3 \mathrm{~min}$ to diffuse into the tissue before retracting the capillary at $0.01 \mathrm{~mm} / \mathrm{s}$. This procedure was repeated for the other hemisphere before sealing the cranial skin with polyacryl glue. Anti-Sedative (Antipemazol $10 \mu \mathrm{l}, \mathrm{NaCl} 0.9 \% 190 \mu \mathrm{l} ; 50 \mu \mathrm{l} / 10 \mathrm{~g}$ ) was injected at least $45 \mathrm{~min}$ post sedation to terminate anaesthesia. 


\subsection{Behaviour}

\subsubsection{Behavioural testing}

Behavioural phenotypes were assessed using a battery of tests comprising a subset of assessments listed below and conducted within tight temporal boundaries. If not stated otherwise, movement was recorded using a camera setup and Videomot 2 software (TSE). Plastic surfaces were thoroughly cleansed with $70 \% \mathrm{EtOH}$ to remove olfactory traces after each subject. All tests were performed serially for individual mice.

\subsubsection{Open field test}

To asses motility and anxiety like behaviour, animals were tested in the Open Field (Hall 1934). In brief, Animals were individually placed in a uniform, grey arena $(40 \times 50 \times 40 \mathrm{~cm})$ and allowed to explore the environment for $3 \mathrm{~min}$. For analysis the Arena was virtually divided into 16 identical squares of which presence in the central 4 were scored as exposed and compared relative to quadrants proximate to the wall. Prolonged time spent in exposed areas was considered as a readout for reduced anxiety given comparable motility.

\subsubsection{Novel object recognition}

To test for both long term memory (LTM) and short term memory (STM) (Ennaceur and Delacour 1988), animals were subjected to the Novel Object Recognition test on the day after the open field test. Subjects were placed in the same grey open filed arena $(40 \times 50 \times 40 \mathrm{~cm})$ as used for the open field test. This time containing 2 identical objects $12 \mathrm{~cm}$ away from proximate walls for habituation and allowed to explore freely for $3 \mathrm{~min}$. Subsequently, animals were returned to the home cage and allowed to rest for $5 \mathrm{~min}$. To test STM one of two identical objects was exchanged with a novel object of different shape, texture and colour. Now the mouse was returned to the arena and again allowed to freely explore both objects. Increased percent of time spent at the novel object was taken as a indicator for STM. Analogous LTM was tested $24 \mathrm{~h}$ after STM by subjecting the individual to another novel object. Here, too the increased percent of time spent at the novel object was taken as an indicator for LTM. Number and duration of investigations at either of the objects was scored manually during the trails using Videomot 2.

\subsubsection{Elevated plus maze}

Anxiety like behaviour was assessed in the Elevated plus maze (Pellow, Chopin et al. 1985), a uniformly grey cross maze with two open and two walled (closed) arms $10 \times 40 \mathrm{~cm}$ each with walls $20 \mathrm{~cm}$ high. Individual animals were placed in the centre of the elevated plus maze, nose facing the closed arm opposite of the operator. Time spent in open arms and centre was scored as time exposed and taken as a measure of anxiety relative to time spent in walled arms. 


\subsubsection{Morris water maze}

To assess hippocampus dependent spatial learning and memory, the Morris water maze was conducted (Sutherland and Rudy 1988). Animals were placed into a pool (1.2m diameter) with opaque water $\left(22^{\circ} \mathrm{C}\right)$ and four distinct optical cues (one in each quadrant). The target quadrant contained a clear plastic platform at a fixed location submersed by $0.5 \mathrm{~cm}$, hence invisible to tested animals. Each trial started when the subject was placed at one of the randomly alternating visual cues. It ended either if the animal rested $2 \mathrm{~s}$ on the target platform or after 1 min if the animal failed to complete the task. In the latter case the animal was guided to the platform and allowed to rest for $30 \mathrm{~s}$. This procedure was repeated four times per day, starting at a different visual cue at each trial. Animals were trained until the first cohort robustly displayed an average latency to find the hidden platform (escape latency) of less than 20 s. Then, on the subsequent day, a probe test was performed, during which the submersed platform was removed. The probe test started when the animal was placed in the quadrant distal to the former platform area. The animal was then allowed to explore the maze freely for 1 min after which it was removed from the maze and returned to the home cage.

Time spent in the target quadrant, crossings of the platform area and search strategies were taken as a read out for spatial memory. Alternatively, training was prematurely terminated, if none of the cohorts showed clear indications of learning after 8 days.

\subsubsection{Barnes maze}

To further test for hippocampus dependent spatial memory, Barnes maze was employed (Barnes 1979). Besides the advantage of an additional spatial memory test, the barnes maze is less stressful to the animal allowing the detection of more subtle phenotypes, given a certain curiosity and motility (Whishaw and Tomie 1996). The barnes maze is composed of a white circular PVC plate (diameter $92 \mathrm{~cm}$ ) with 20 holes located around the rim and 4 distinct visual cues, one located at each quadrant. The central hole in the quadrant distal to the operator entry site contained an escape cage, while maintaining identical appearance compared to other holes. To increase aversion of the maze and hence increase motivation to find the escape cage, the maze was brightly lit using 4x60W Light bulbs and 2xLED flood lights (30W) positioned above the maze.

The Training protocol was adapted from Attar and colleges (Attar, Liu et al. 2013) with several adjustments. In brief, the protocol is separated into three stages habituation, training, and testing. On day one animals were placed in the centre of the maze into a clear PVC tube $(15 \mathrm{~cm}$ diameter) for $15 \mathrm{~s}$ to habituate the mouse to the environment. Then the subject was slowly guided to the target hole by gently moving the tube. The animal was allowed to explore the target for $3 \mathrm{~min}$. If it did not enter the escape cage, it was gently nudged with the PVC tube to enter. Once in the escape cage the subject was allowed to rest for $1 \mathrm{~min}$ before returning to home cage. On consecutive days 2-4 individuals were placed in the centre and covered with a grey cylinder for $15 \mathrm{~s}$ to randomise start orientation. Subsequently, mice were allowed to explore 
the maze for $2 \mathrm{~min}$. If the target was not entered, mice were again nudged to do so. After entering target they were allowed to rest for $1 \mathrm{~min}$. On day 5 a probe test was performed during which the escape cage was removed. Mice were again placed into a grey cylinder in the centre of the maze for 15s. After release subjects were given $2 \mathrm{~min}$ to explore the maze. Time spent in target area or target quadrant was evaluated and taken as a read out for spatial memory as indicated for each experiment. If one of the tested cohorts did not show significantly increased time spent in target, the training was prolonged for additional 4 training days and one subsequent probe test.

\subsubsection{RotaRod}

To assess motor coordination and balance, mice were subjected to the TSE RotaRod test. Subjects were placed on a rotatable rubber coated bar above a metal grid. During training the bar rotated at $5 \mathrm{rpm}$ for $180 \mathrm{~s}$. If the mouse fell off it was placed back for the time remaining. During motor test animals were placed on the rod, which gradually accelerated during $180 \mathrm{~s}$ from 5 to $40 \mathrm{rpm}$. Latency to fall was measured using TSE RotaRod Advanced software and indicative of motor function.

\subsubsection{Forced swim test}

To asses despair the forced swim test was used (Porsolt, Le Pichon et al. 1977). Animals were placed for $3 \mathrm{~min}$ into a $5 \mathrm{~L}$ beaker filled with $20 \mathrm{~cm}$ water to avoid that subjects could touch the ground. Animals were recorded in 1080p (iPhone 6s, Apple) and floating subsequently manually scored as the absence of movement other than stabilisation strokes using Quicktime (Apple) and Stopwatch (Apple).

\subsubsection{Contextual fear conditioning}

Classical pavlovian conditioning was performed in a NIR Video Fear Conditioning system (Med Associates Inc.). The system features a solid, sound proofed chamber $(58 \mathrm{~cm} \times 30 \mathrm{~cm} \times 27 \mathrm{~cm}$, length $\mathrm{x}$ width $\mathrm{x}$ height) with lighting (12V) and white noise generator. A clear plexiglas cage (35 $\mathrm{cm} \times 20 \mathrm{~cm} \times 20 \mathrm{~cm}$, length $x$ width $x$ height) featuring an electrifiable, removable grid floor is located inside the chamber. Lighting, noise and electrical stimulus were automatically controlled by the NIR Video Fear Conditioning software according to operator settings. Analogous to the classical pavlovian paradigm the conditioned stimulus is presented as cage environment (CS) and the unconditioned stimulus (US) as foot shock. On the first day (training) animals were allowed to freely explore the camber for $3 \mathrm{~min}$, while white noise was played. An electrical foot shock $(0.5 \mathrm{~mA})$ was applied for the last $2 \mathrm{~s}$ of each trial. On the second day (recall) animals were again allowed to freely explore the chamber for $3 \mathrm{~min}$ without the application of a foot shock. Light was switched on and animals were recorded during the entire trial. Behaviour was assessed in linear analysis mode and considered freezing if the Motion Index was below a 
threshold of 18 for at least 30 frames. Scoring was done automatically with the NIR Video Fear Conditioning software provided with the system.

\subsubsection{Intracardial perfusion}

Animals were anaesthetised with a lethal dose of Ketamin/Xylazine (Ketamin $20 \mu \mathrm{l}$, Xylarem $150 \mu \mathrm{l}, \mathrm{NaCl} 0.9 \% 30 \mu \mathrm{l}, 100 \mu \mathrm{l}$ per $10 \mathrm{~g}$ body weight). After being reflex irresponsive subjects were fixated with needles on a dissection mat. The ribcage was opened and heart was exposed. Now the right ventricle was punctured, while the left ventricle was perfused with ice cold PBS using a peristaltic pump at $5 \mathrm{rpm}$ for $6 \mathrm{~min}$ (Heidolph, Pupmdrive 5201). Subsequently, PBS was exchanged for ice cold $4 \%$ PFA in PBS which was injected for additional 5 min. After perfusion the brain was extracted and post-fixed in $4 \%$ PFA for $24 \mathrm{~h}$. For cryo- protection the brain was subjected to $10 \%, 20 \%$, and $30 \%$ Sucrose/PBS for $24 \mathrm{~h}$ each and subsequently embedded in Jung tissue freezing medium (Leica Microsystems) for cryosectioning at $30 \mu \mathrm{m}$ in a Leica CM 1850 UV cryostat at $-20^{\circ} \mathrm{C}$.

\subsection{Molecular cloning}

\subsubsection{Molecular cloning of AnxA2}

To generate an AAV based overexpression construct AnxA2 was amplified from mouse cDNA using custom made AnxA2_perfectmatch primer (sequence see Table 3.1) and Fusion HighFidelity DNA Polymerase (NEB) according to manufacturer's protocol. The PCR product was purified by $0.6 \%$ agar (Roth) gel-electrophoresis in TAE-buffer $(40 \mathrm{mM}$ Tris-acetate, $1 \mathrm{mM}$ EDTA) followed by extraction with QIAquick Gel Extraction Kit (Qiagen) and ligated into pGEM-T easy (Promega) according to manufacturer's protocol. The plasmid was then transformed into Sure2 cells for Plasmid preparation. The resulting Plasmid DNA was subjected to PCR using AnxA2_Nhe1_F and AnxA2_BamH1_R (sequence see Table 3.1) to add feasible restriction sites and mutate the stop codon to be able to generate fusion proteins. The PCR product was purified as previously described and digested with BamHI-HF and Nhe1-HF (NEB) at $37^{\circ} \mathrm{C}$ for $15 \mathrm{~min}$ and inactivated at $80{ }^{\circ} \mathrm{C}$ for $20 \mathrm{~min}$. The digested construct was ligated into an

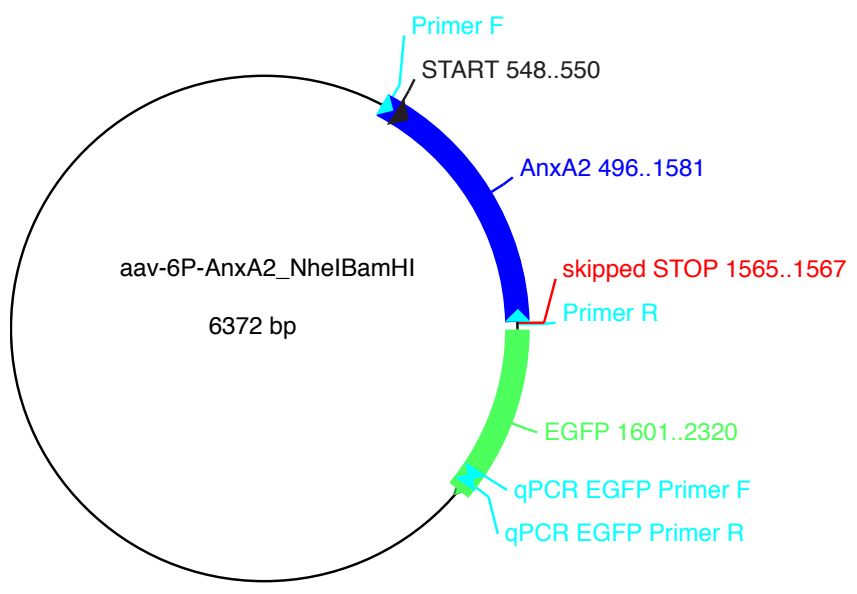

Figure 3.2: Illustration of AAV-6PAnXA2GFP-NoTB-SEWB.

Notably, the AnxA2 overexpression construct features a skipped STOP codon to generate the GFP fusion protein. 
accordingly digested AAV-6P-GFP-NoTB-SEWB, a custom made AAV plasmid featuring the synapsin promotor and GFP sequence. Constructs were ligated using T4 DNA Ligase (NEB) according to manufacturer's protocol. The ligation product (AAV-6P-AnxA2/GFP-NoTB-SEWB) was transformed in Sure2 cells, amplified, and validated by restriction digest and Sanger sequencing (economy barcode run, SeqLab. The resulting in the plasmid is illustrated in Figure 3.2.

\subsubsection{Bacterial transformation}

For transformation of plasmids a $20 \mu$ aliquot of SURE 2 Supercompetent Cells (Agilent) was thawed on ice, and transformed according to manufacturer's guidelines. In brief, bacteria were supplied with $0.1-50 \mathrm{ng}$ of desired plasmid and incubated for $30 \mathrm{~min}$ on ice. Subsequently, bacteria were heat pulsed at $42{ }^{\circ} \mathrm{C}$ in a ThermoMixer (Eppendorf) for exactly $30 \mathrm{~s}$. Immediately afterwards, the sample was transferred to ice and chilled for $2 \mathrm{~min}$. Then $500 \mu \mathrm{l}$ of $37^{\circ} \mathrm{C}$ LBbroth were added and incubated at $37^{\circ} \mathrm{C}$ for 1 hour while shaking at $250 \mathrm{rpm}$. Then $200 \mu \mathrm{l}$ and $20 \mu \mathrm{l}$ (high and low density plating) of the transformation mixture were applied to separate LB agar (AppliChem) plates containing Ampicillin as antibiotic and dispersed using glass beads. Colony growth was checked after plates were incubated at $37^{\circ} \mathrm{C} O / \mathrm{N}$.

\subsubsection{Plasmid isolation}

After O/N incubation of bacteria on LB-Agar+Ampicillin colonies were picked using $10 \mu$ pipette tips. Each colony was incubated o/n at $37^{\circ} \mathrm{C}$ and $160 \mathrm{rpm}$ in $8 \mathrm{ml}$ LB-broth containing Ampicillin as antibiotic in a New Brunswick Innova 44 incubator. Bacteria were harvested at $4000 \mathrm{~g}$ at $4{ }^{\circ} \mathrm{C}$ for $10 \mathrm{~min}$, then plasmids were isolated using QIAprep Spin Miniprep Kit (Qiagen) following the manufacturer's instructions. Plasmid sequences were first validated by restriction digest and secondly by Sanger sequencing (economy barcode run, SeqLab). After successful plasmid validation, large over-night cultures were set up. To generate sufficient amounts for viral production 2-8 L of LB-broth (AppliChem) plus Ampicillin were incubated o/n at $37^{\circ} \mathrm{C}$ and $160 \mathrm{rpm}$ in a New Brunswick Innova 44 incubator. Subsequently, cultures were precipitated at $6000 \mathrm{~g}$ for $15 \mathrm{~min}$ at $4{ }^{\circ} \mathrm{C}$ in a RC6 (Sorvall). Plasmids were isolated using NucleoBond ${ }^{\circledR}$ Xtra Midi Kit (Macherey-Nagel) following the manufacturer's instructions. Isolated plasmids were again validated by restriction digest and Sanger sequencing.

\subsubsection{Adeno-associated-viral particle (AAV) production}

Adeno-associated virus preparation was adapted from McClure and colleagues (McClure, Cole et al. 2011) with several adjustments. Required Plasmids (pRV1, pH21, pF $\Delta 6$ ) were amplified and extracted by MidiPrep as described before and digested for sequence validation (pRV1(Xbal) 7.5 kb, 3.8 kb; pH21(EcoRl) 4.5 kb, 2.8 kb, 0.2 kb; pF 66 (Hindlll) 5.5 kb, 3 kb, $3 \mathrm{~kb}, 2.3 \mathrm{~kb}, 1.5 \mathrm{~kb}$ ). For viral particle assembly five HEK293T $14.5 \mathrm{~cm}$ dishes were grown to $70-80 \%$ confluence in standard DMEM with low glucose containing $10 \%$ foetal bovine serum 
(Gibco) and $100 \mathrm{U} / \mathrm{ml}$ penicillin/ $100 \mu \mathrm{g} / \mathrm{ml}$ streptomycin. 3 hours before transfection medium was removed and replaced with fresh medium. For one batch $(5,14.5 \mathrm{~cm}$ dishes) $62.5 \mu \mathrm{g}$ AAV plasmid, $125 \mu \mathrm{g} \mathrm{pF} \Delta 6,31.25 \mu \mathrm{g} \mathrm{pRV} 1,31.25 \mu \mathrm{g} \mathrm{pH} 21$ and $1650 \mu \mathrm{l} 2.5 \mathrm{M} \mathrm{CaCl} 2$ were mixed in $12 \mathrm{ml} \mathrm{dH} 2 \mathrm{O}$ and sterile filtered. Whilst vortexing $13 \mathrm{ml} 2 x$ HEPES $(250 \mathrm{mM} \mathrm{NaCl}, 1.5 \mathrm{mM}$ $\mathrm{Na}_{2} \mathrm{HPO}_{4}, 50 \mathrm{mM}$ HEPES, $\mathrm{pH}$ 7.05) were quickly added to the solution and heavily vortexed. The solution was allowed to stand for $2 \mathrm{~min}$ until a white precipitate started to form. Now $5 \mathrm{ml}$ of the transfection solution was added to each $14.5 \mathrm{~cm}$ culture dish. Depending on expression cells were incubated for $48 \mathrm{~h}-72 \mathrm{~h}$. For harvest, the supernatant was removed from plates. Cells were then washed and scraped from the dish. Following, the samples were centrifuged for $5 \mathrm{~min}$ at $1000 \mathrm{~g}, 4^{\circ} \mathrm{C}$ and resuspended in lysis buffer ( $20 \mathrm{mM}$ Tris, $150 \mathrm{mM} \mathrm{NaCl}, \mathrm{pH} 8.0,0.5 \%$ NaDOC, Benzonase). The slurry was then incubated for $30 \mathrm{~min}$ at $37^{\circ} \mathrm{C}$ and occasional vigorous shaking. Reaction was quenched by adding $21.63 \mathrm{mg} / \mathrm{ml} \mathrm{NaCl}$ and incubating at $56{ }^{\circ} \mathrm{C}$ for $30 \mathrm{~min}$. After one freeze thaw cycle $\left(-80^{\circ} \mathrm{C}\right.$ freezing $\mathrm{O} / \mathrm{N}, 37^{\circ} \mathrm{C}$ thawing) the sample was centrifuged for $30 \mathrm{~min}$ at $4000 \mathrm{~g}$ and subsequently layered onto a discontinuous lodixanol (Sigma) gradient (top to bottom $6.5 \mathrm{ml}, 15 \% ; 4 \mathrm{ml}, 25 \% ; 3 \mathrm{ml}, 40 \% ; 3 \mathrm{ml}, 54 \%$ ) in a ultracentrifuge tube. To avoid collapse of the ultracentrifuge tubes, any air volume was filled with with PBS-MK. Then viral preparation was centrifuged for $1.5 \mathrm{~h}$ at $60.000 \mathrm{rpm}$ (rotor: $\mathrm{Ti} 70$, Beckmann). Subsequently the $40 / 54 \%$ interphase was removed, diluted to $10 \mathrm{ml}$ using PBSMK and concentrated in an Amicon $100 \mathrm{~K}$ (Millipore) at $2000 \mathrm{~g}$ and RT. The samples were centrifuged repeatedly for 5 min until $500 \mu$ resided in the concentration unit . The concentration was repeated 3 times by refilling the concentrator to $10 \mathrm{ml}$ with PBS-MK. Subsequently, the viral solution was sterile filtered and stored at $4^{\circ} \mathrm{C}$ and $-80^{\circ} \mathrm{C}$ for longterm storage respectively.

\subsection{Cell culture}

\subsubsection{Dissociated primary neuronal culture}

Prior to dissection culture dishes were coated with Poly-D-lysine (Sigma) $0.5 \mathrm{mg} / \mathrm{ml} \mathrm{O/N}$ at RT or alternatively at $37{ }^{\circ} \mathrm{C}$ for $1 \mathrm{~h}$. Plates were then washed 3 times with ddH2O. Pregnant females were sacrificed at E17.5, embryos were extracted, anaesthetised in ice cold PBS, and immediately decapitated. Depending on experimental approach hippocampi or cortices were dissected and transferred into $15 \mathrm{ml}$ falcon on ice cold PBS. After dissection ice cold PBS was removed and $4.5 \mathrm{ml}$ warm PBS and $500 \mu \mathrm{l}$ with $2.5 \%$ trypsin were added to the tissue under a sterile bench and incubated in a water bath on $37^{\circ} \mathrm{C}$ for $13 \mathrm{~min}$ for digestion. Subsequently, 5 $\mathrm{ml}$ processing medium (PM) (DMEM, $10 \% \mathrm{FBS}, 1 \% \mathrm{P} / \mathrm{S}$ ) were added to quench the digestion. The sample was then supplemented with $10 \mu \mathrm{l}$ DNAse to degrade released DNA, the tube was inverted once and the tissue allowed to sediment to the bottom before removal of medium. The digested tissue was washed three times with PM without disturbing the tissue. After washing, medium was removed and tissue homogenised in $1 \mathrm{ml}$ PM by carefully pipetting the chunks up and down in a $1 \mathrm{ml}$ pipette (5-10 strokes). Cells were then precipitated at $200 \mathrm{~g}, 37^{\circ} \mathrm{C}$ for $5 \mathrm{~min}$ 
and resuspended in maintenance medium (MM) (Neurobasal, Gibco; $2 \%$ B27, Gibco; $1 \%$ Penicillin/Streptomycin, AppliChem; $1 \%$ GlutaMAX,Gibco). Subsequently, neurons were plated at $6,84 \times 10^{4} \mathrm{cells} / \mathrm{cm}^{2}$ (hippocampal) or $15.3 \times 10^{4}$ cells $/ \mathrm{cm}^{2}$ (cortical). Cells were maintained in a $\mathrm{CO} 2$ cell culture incubator (New Brunswick/Binder) and $30 \%$ medium were changed twice weekly.

\subsubsection{Chemical neuronal stimulation}

Neuronal cultures were stimulated on day in vitro (DIV) 10 with a final concentration of $20 \mathrm{mM}$ $\mathrm{KCl}, 50 \mu \mathrm{M}$ NMDA or $200 \mu \mathrm{M} \mathrm{H} 2 \mathrm{O} 2$ in equal volumes to mimic specific physiological challenges. Stimulation was applied for $10 \mathrm{~min}$, then medium was removed and cells gently washed with DPBS. Immediately after washing conditioned medium was applied and cells incubated for an additional 10 min for recovery. Afterwards, medium was removed and plates washed once with DPBS, then $100 \mu \mathrm{l}$ TRIzol (Thermo Fisher) were added and the cells harvested and processed as described in "3.5.6.RNA isolation".

\subsubsection{Dissociated primary neuronal SILAC culture}

To establish stable isotope labelling with amino acids in cell culture (SILAC) in post mitotic primary neurons neuronal cultures were produced as described in "3.4.1 Dissociated primary neuronal culture". Dissociated primary neuronal culture. Only cortical neurons were seeded at a density of $15.3 \times 10^{4}$ cells $/ \mathrm{cm}^{2}$ in $14.5 \mathrm{~cm}$ culture dishes. Additionally, MM was replaced by SILAC medium containing either light or heavy Arginine and Lysine (L-Lysine- ${ }^{13} \mathrm{C}_{6},{ }^{15} \mathrm{~N}_{2}$, LArginine- ${ }^{13} \mathrm{C}_{6},{ }^{15} \mathrm{~N}_{4}$; Euroisotop) as follows SILAC F12:DMEM (Pierce), $0.388 \mathrm{mM}$ L-Arginine, 0.798 mM L-Lysine , 2 \% B27, 1 \% Penicillin/Streptomycin, $1 \%$ GlutaMAX. To achieve sufficient labelling $22 \times 10^{6}$ cortical neurons were plated per $14.5 \mathrm{~cm}$ dish in $20 \mathrm{ml}$ of SILAC medium. After 3 days, $6 \mathrm{ml}$ of medium were replaced with $6 \mathrm{ml}$ of fresh SILAC medium. This was repeated every 2 days until DIV10 after which labelled nuclear protein lysates were isolated (cf. "3.5.1 SILAC nuclear protein isolation").

\subsubsection{Heterogenous primary neuronal cultures}

After preparing a dissociated hippocampal cell suspension from E17.5 embryos as described above, cells were diluted to $10 \mathrm{ml} \mathrm{PM}$ and then precipitated at $200 \mathrm{~g}$ at $37^{\circ} \mathrm{C}$ for $5 \mathrm{~min}$. Cells were then resuspended in $500 \mu$ l Hibernate E (Gibco) with $2 \%$ B27 and $1 \%$ P/S and split depending on number of infection constructs into $1.5 \mathrm{ml}$ centrifuge cups. $20 \mu \mathrm{l}$ of concentrated AAVs carrying the desired construct were added to each cell aliquot. Infection samples were then incubated for $2 \mathrm{~h}$ at $70 \mathrm{rpm}, 37^{\circ} \mathrm{C}$ in shaking incubator. Afterwards, cells were resuspended in $1 \mathrm{ml} \mathrm{MM}$ and further diluted in $10 \mathrm{ml} \mathrm{MM}$, then centrifuged $5 \mathrm{~min}$ at $300 \mathrm{~g}$ and $37^{\circ} \mathrm{C}$. Subsequently, cells were washed twice in $10 \mathrm{ml} \mathrm{MM}$. The supernatant was removed and cells 


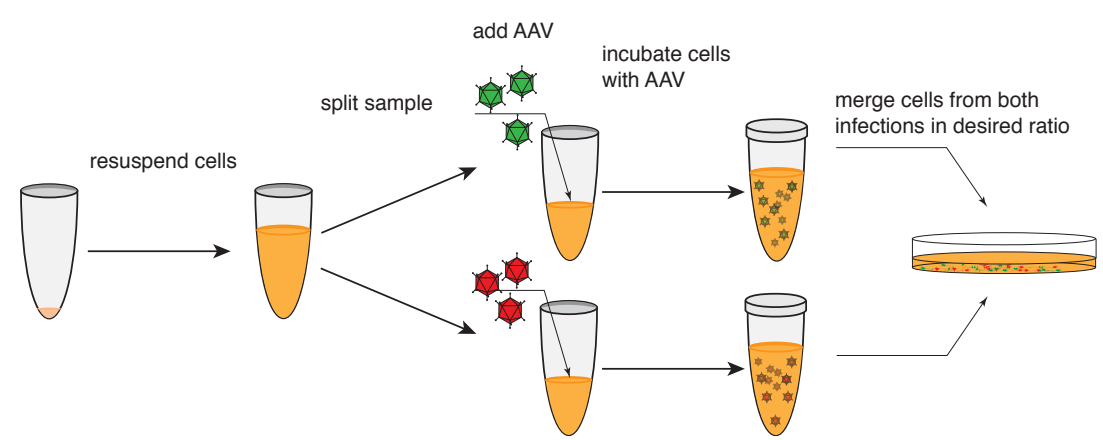

Figure 3.3: Production of heterogenous PNC.

Cells are resuspended from pellet split into different tubes and infected with the desired construct, delivered by an AAV. Finally, the infected cells are seeded in desired ratios.

diluted in MM containing $100 \mu \mathrm{M}$ heparin (sigma) to avoid AAV propagation (Mietzsch, Broecker et al. 2014) and plated at $6,8 \times 10^{4}$ cells $/ \mathrm{cm}^{2}$ in 6 well dishes, as illustrated in figure 3.3 .

\subsubsection{Optogenetic stimulation}

Neurons were either infected with AAV1/2-ChiEF-tdTomato for optogenetic stimulation (primary stimulated neurons) or with AAV1/2-FOS-eYFP as activity reporter (secondary stimulated neurons). Optogenetic stimulation was performed on DIV10, pulse duration and inter stimulation intervals (ISI) were adapted from to Schoenenberger and colleagues (Schoenenberger, Gerosa et al. 2009) using a custom built stimulator. In brief, the stimulation was conducted inside a Galaxy $170 \mathrm{R}$ cell culture incubator (New Brunswick). Culture dishes containing neurons were placed on the stimulation unit $30 \mathrm{~min}$ prior to stimulation and then left to rest to avoid mechanical stimulation. The protocol was then initiated using a remote control. During the stimulation 300 ms pulses alternated with $1 \mathrm{~s}$ ISI for 10 repetitions if not stated otherwise. Neurons were allowed to rest for $30 \mathrm{~min}$ before further preparation. AAV-oChIEF-tdTomato plasmid was a gift from Roger Tsien (Addgene plasmid \# 50977), pOTTC476 - pAAV c-fos eYFP was a gift from Brandon Harvey (Addgene plasmid \# 47907).

\subsubsection{Single cell sorting}

After optogenetic stimulation (see Optogenetic stimulation) medium was removed carefully and cells wash once with $37^{\circ} \mathrm{C}$ DPBS. Subsequently, $200 \mu$ l Neuron Isolation Enzyme (with papain) from Pierce ${ }^{\mathrm{TM}}$ Primary Cell Isolation Kit (Thermo) was added and incubated for 5 min at $37^{\circ} \mathrm{C}$. The dish was then put on ice, $1 \mathrm{ml}$ ice cold sorting buffer (DPBS, $2 \%$ FBS) was added and cells transferred into $14 \mathrm{ml}$ centrifuge tube. Suspension was pipetted 5 times up and down to separate the cells and filled up to $10 \mathrm{ml}$ with sorting buffer. Neurons were precipitated for $5 \mathrm{~min}$ at $4{ }^{\circ} \mathrm{C}, 200 \mathrm{~g}$. Afterwards, the supernatant was removed and cells carefully resuspended in $10 \mathrm{ml}$ sorting buffer. This washing step was repeated once before re-suspending cells in $500 \mu \mathrm{l}$ sorting buffer. Before sorting viability was assessed for $10 \mu$ of the suspension using Trypan blue in a 1:1 dilution (Sigma). Now neurons were sorted directly into lysis buffer to avoid transcriptional changes. Sorting was based on the ectopically expressed fluorescence tag 
introduced by AAV infection (see Heterogenous primary neuronal cultures) using a FACS ARIA III (BD Bioscience). If not stated otherwise, samples were kept at $4{ }^{\circ} \mathrm{C}$.

\subsubsection{Anle138b treatment}

Primary hippocampal cultures were produced as mentioned beforehand. On DIV9 cells were treated either with $1 \mu \mathrm{M}$ anle138b or $10 \mu \mathrm{M} \mathrm{AB1-40}$ and incubated for $24 \mathrm{~h}$ in a CO2 cell culture incubator (New Brunswick/Binder). Subsequently, anle138b pre-treated cultures were supplemented with $10 \mu \mathrm{M} A B 1-40$, while $\mathrm{A} B 1-40$ pre-treated cultures were supplemented with 1 $\mu \mathrm{M}$ anle138b. After 48 hours the cultures were subjected to either CyQuant or MTT assay.

\subsubsection{JS28 treatment}

JS28 was a kind gift from Johanna Senger and Prof. Dr. Manfred Jung, from the Pharmazeutische und Medizinische Chemie of the Albert-Ludwigs-Universität Freiburg. For treatment the compound was dissolved in $\mathrm{ddH}_{2} \mathrm{O}$ and applied at the indicated concentrations on DIV10. After $24 \mathrm{~h}$ cultures were harvested and protein extracted according to protocol (cf. "3.5.2. Total protein extraction") and tubular acetylation quantified using Western blot (cf. "3.5.5. Western blotting') and anti-acetylated Tubulin antibody (T7451, Sigma Aldrich).

\subsubsection{Metabolic rate assay}

Metabolic rate of neurons in cell culture was assayed using 3-(4,5-dimethylthiazol-2-yl)-2,5diphenyltetrazolium bromide (MTT, Sigma-Aldrich) as adapted from Mosmann (Mosmann 1983). The assay is based on the metabolic conversion of MTT (bright yellow) to its formazan (purple), i.e. for a high metabolic rate the absorption at $570 \mathrm{~nm}$ is increased and vice versa. MTT was added to cell culture at a final concentration of $0.5 \mathrm{mg} / \mathrm{ml}$ and subsequently incubated for $1 \mathrm{~h}$ at $37^{\circ} \mathrm{C}$. Then MTT containing medium was removed and the resulted formazan dissolved in DMSO by shaking for 15 min while protected from light. Absorption at $570 \mathrm{~nm}$ was then assessed using a Infinite 200 PRO (Tecan) or FLUOstar Omega (BMG Labtech). Results were extracted using Excel (Microsoft) and illustrated using Prism 7 (GraphPad) statistical significance was tested using t-test for condition comparison or one-way ANOVA for group comparisons.

\subsubsection{Membrane integrity assay}

To asses membrane integrity of neurons in cell culture CyQUANT Direct assay (Thermo Fisher) was conducted according to manufacturer's instructions. The kit is composed of two stains, the first being a fluorescent stain for DNA which penetrates membranes. The second dye absorbs fluorescent activity of dye one, however is not able to penetrate intact but only leaky membrane. After 30min incubation fluorescence was measured with a Tecan infinite 200 for AB 1-40 or FLUOstar Omega (BMG Labtech) for Aß1-42 (-D,-L) and aSynuclein. Results were extracted 
and analysed using Excel (Microsoft) and displayed using Prism 7 (GraphPad) statistical significance was tested using t-test for condition comparison or one-way ANOVA for group comparisons.

\subsection{Molecular Analysis}

\subsubsection{In-silico oligo nucleotide design}

Oligonucleotides for cloning, PCR, and Sybr Green qPCR were designed using ApE v2.0.47 (Wayne Davis W. http://biologylabs.utah.edu/jorgensen/wayned/ape/) for target assembly. OligoCalc was used to calculate melting Temperature (http://biotools.nubic.northwestern.edu/ OligoCalc.html) and in-silicoPCR (Kent, J. http://genome.ucsc.edu/cgi-bin/hgPcr) was used to control for off-targets in case of primers. Alternatively, for qPCR the UPL Assay Design Centre (Roche) (https://lifescience.roche.com/en de/brands/universal-probe-library.html) was used to design UPL qPCR Primers. All oligo nucleotides were synthesised by Sigma Aldrich.

\subsubsection{Total protein extraction}

Total protein was extracted from tissue by homogenisation with a BeadRuptor 24 (Onmi International) in 2 tissue volumes Tx Buffer+SDS ( ( $0.2 \mathrm{M}$ Tris base, $1.5 \mathrm{M} \mathrm{NaCl}, 0.05 \%$ Tween $20,1 \%$ SDS). Samples were then incubated for $10 \mathrm{~min}$ on rotating wheel at $4{ }^{\circ} \mathrm{C}, 20 \mathrm{rpm}$ and subsequently sent to Bioruptor Plus (Diagenode) $15 \mathrm{~min}$ (30sec on/off cycle, $4{ }^{\circ} \mathrm{C}$ ). Samples were now centrifuged for $10 \mathrm{~min}$ at $10,000 \mathrm{~g}$ and $4{ }^{\circ} \mathrm{C}$ to precipitate residual debris. The supernatant containing total protein was then transferred into a new tube.

\subsubsection{Nuclear protein enrichment}

For nuclear Protein enrichment tissue was homogenised in a BeadRuptor 24 (Onmi International) with 2 tissue volumes Tx Buffer (0.2 M Tris base, $1.5 \mathrm{M} \mathrm{NaCl}, 0.05 \%$ Tween 20) then put on a rotating wheel for $10 \mathrm{~min}$ at $4{ }^{\circ} \mathrm{C}$ to allow swelling of the cells and subsequently centrifuged for $10 \mathrm{~min}$ at $2,000 \mathrm{rpm}, 4^{\circ} \mathrm{C}$. The supernatant was then transferred to a new tube (cytosolic fraction) and the pellet washed once in Tx buffer. After washing, supernatant was discarded, 1 tissue volume Tx Buffer $+1 \%$ SDS added, and samples incubated on rotating wheel for $5 \mathrm{~min}$ at $20 \mathrm{rpm}, 4{ }^{\circ} \mathrm{C}$. Subsequently, samples were sent to Bioruptor Plus (Diagenode) for $15 \mathrm{~min}$ (30 sec on/off cycle, $4^{\circ} \mathrm{C}$ ) and then centrifuged for $10 \mathrm{~min}$ at 10,000 g, 4 ${ }^{\circ} \mathrm{C}$ to remove residual debris. Afterwards, the supernatant was transferred into a fresh tube (nuclear enriched protein).

\subsubsection{Sub-cellular fractionation}

To understand the sub cellular localisation of proteins, cells and tissue were separated into membrane, cytoplasmatic, cytoskeleton, nuclear, and chromatin bound fraction. To do so, protein extraction was carried out using the Subcellular Protein Fractionation Kit for Cultured 
Cells (Thermo scientific) according to manufacturer's instructions. Procedure deviated from protocol if the kit was used to extract protein from tissue. Here, samples were first homogenised in $300 \mu \mathrm{l} \mathrm{PBS}+$ Proteinase inhibitors (Roche) following centrifugation at $2000 \mathrm{~g}, 4^{\circ} \mathrm{C}$. The supernatant was discarded and started from step 1 according to manufacturer's protocol.

\subsubsection{SILAC nuclear protein isolation}

To isolate nuclear protein in a low detergent environment cells were first rinsed twice in ice cold DPBS (Pan Biotech) then scraped off the dish in $2 \mathrm{ml}$ fresh DPBS into a protein LoBind micro centrifuge tube (Eppendorf). Cell samples were the centrifuged for $5 \mathrm{~min}$ at $700 \mathrm{~g}$ on $4{ }^{\circ} \mathrm{C}$ and the supernatant discarded. Then Packed cell volume was estimated (PCV) and 5x PCV lysis buffer (10 mM HEPES, pH $7.91 .5 \mathrm{mM} \mathrm{MgCl}$, $10 \mathrm{mM} \mathrm{KCl2,} 0$.3 M Sucrose,1 mM DTT, 1x Protease Inhibitor Cocktail, $0.15 \mu \mathrm{L}$ TSA, $10 \mu \mathrm{L}$ NAD) were added. The cell pellet was then gently resuspended to avoid foam formation and then incubated for $15 \min$ on $4{ }^{\circ} \mathrm{C}$ to allow the cells to swell. Afterwards samples were centrifuged for $5 \mathrm{~min}$ at $420 \mathrm{~g}$ on $4{ }^{\circ} \mathrm{C}$ after which the supernatant was removed and the pellet resuspended in 2x PCV fresh lysis buffer. Cells were now homogenised using a narrow-gauge No. 27 (B.Braun) hypodermic needle in 15 strokes. Subsequently, lysis was checked under the microscope using trypan blue (Sigma) as stain. If homogenisation was not satisfactory the samples were given an additional 5 strokes until lysed. Samples were then centrifuged for $20 \mathrm{~min}$ at $10000 \mathrm{~g}$ on $4{ }^{\circ} \mathrm{C}$ and the supernatant containing the cytosolic fraction transferred to a new protein LoBind tube. The residual nucleic pellet was resuspended in 2/3 x PCV extraction buffer ( 20 mM HEPES, pH $7.91 .5 \mathrm{mM} \mathrm{MgCl}, 0.42 \mathrm{M}$ $\mathrm{NaCl}, 0.2 \mathrm{mM}$ EDTA, 25\% (v/v) Glycerol, $0.7 \mathrm{mM}$ DTT, 1x Protease Inhibitor Cocktail, $0.23 \mu \mathrm{L}$ TSA, $15 \mu \mathrm{L}$ NAD) using a narrow-gauge No. 27 (B.Braun) hypodermic needle in 10 strokes. Samples were then gently shook for $30 \mathrm{~min}$ at $4{ }^{\circ} \mathrm{C}$ and afterwards centrifuged for $5 \mathrm{~min}$ at $21000 \mathrm{~g}$ on $4{ }^{\circ} \mathrm{C}$. The resulting supernatant containing soluble chromatin fraction was transferred to a fresh tube and stored at $-80^{\circ} \mathrm{C}$ until used for $\mathrm{H} 4 \mathrm{~K} 12 \mathrm{ac}$ peptide pulldown and subsequent liquid-chromatography mass-spectrometry/mass-spectrometry (LC MS/MS).

\subsubsection{Immunohistochemistry}

To stain for specific protein epitopes in brain tissue, cryo-sections (cf. "3.2.2. Intracardial perfusion") were washed $3 \times 5 \mathrm{~min}$ in PBS to remove cryo-medium. The sections were then permeabilised by incubating twice for $10 \mathrm{~min}$ RT in $0.1 \%$ Triton-X in PBS and subsequently blocked for $60 \mathrm{~min}$ in blocking buffer (PBS, $0.3 \%$ TritonX and $5 \%$ goat serum). Now the primary antibody anti-AnxA2 (1:200, Abcam) was added to fresh blocking buffer and incubated with samples at $4{ }^{\circ} \mathrm{C} \mathrm{O} / \mathrm{N}$. The next day samples were washed three times for $10 \mathrm{~min}$ in PBS with $0.2 \%$ TritonX and $1 \%$ goat serum. The secondary antibody (Alexa488 anti-Rb) was then applied in 1:1000 dilution to the samples in blocking buffer for $2 \mathrm{~h}$ at RT in the dark. Then sections were washed three times in PBS, subsequently stained with DAPI (1:10000 in PBS) for 30 min, washed again twice in PBS and mounted in Mowiol (Roth). 


\subsubsection{Immunocytochemistry}

To perform epitope based antibody staining in cell culture, neuronal cells were seeded on glass coverslips (15 mm, Thermo Fisher) which were previously treated with nitric acid (Roth) O/N, washed 3 times with $\mathrm{H} 2 \mathrm{O}$ and stored in $70 \% \mathrm{EtOH}$ until use. Before seeding, coverslips were coated O/N with $0.5 \mathrm{mg} / \mathrm{ml}$ Poly-D-lysine (Sigma), while rocking. On DIV10 cells were treated as described, then medium was removed and cells gently washed with $37^{\circ} \mathrm{C}$ DPBS (Pan-Biotech). Immediately after washing, 4\% paraformaldehyde (PFA, Roth) was added and samples incubated $10 \mathrm{~min}$ at RT for fixation. Subsequently, fixative was discarded and cells gently washed with DPBS to remove residual PFA. Slides were then permeabilised by incubating for 10 min at RT in $0.1 \%$ Triton-X in PBS and subsequently blocked for 60 min in blocking buffer (PBS, $0.3 \%$ TritonX and $5 \%$ goat serum). Then the primary antibody was added in fresh blocking buffer (1:200 AnxA2, 1:1000 cFOS, 1:500 $\mathrm{\gamma H} 2 \mathrm{Ax}$ ) and incubated at $4{ }^{\circ} \mathrm{C} \mathrm{O} / \mathrm{N}$. The next day slides were washed three times for 10 min in PBS with $0.2 \%$ TritonX and $1 \%$ goat serum. The secondary antibody (Alexa488 anti-Rb, Alexa633 anti-mM) was then applied 1:1000 to the samples in blocking buffer $2 \mathrm{~h}$ at $\mathrm{RT}$ in the dark. Slides were then mounted using Vectrashield with DAPI (Vector Laboratories).

\subsubsection{Confocal imaging}

Confocal microscopy was performed on a ZEISS LSM 710 (Zeiss) 5x or 63x oil objective. Image acquisition was done using ZEN lite (ZEISS).

\subsubsection{Western blotting}

For detection of protein levels SDS-PAGE with nitrocellulose membrane transfer and immunostaining "Western blot" was performed. Protein samples (10-50 $\mu \mathrm{g})$ containing Protein inhibitor cocktail (Roche) were diluted 1:5 in loading buffer $(250 \mathrm{mM}$ Tris $\cdot \mathrm{HCl}, \mathrm{pH} \mathrm{6.8,} 10 \%$ SDS, $30 \%$ Glycerol, $10 \mathrm{mM} \mathrm{DTT}, 0.05 \%$ Bromophenol Blue) heated to $95{ }^{\circ} \mathrm{C}$ for $5 \mathrm{~min}$ and then briefly chilled on ice. Subsequently, samples were either loaded on self casted $12 \%$ polyacrylamide SDS-PAGE gels, or Mini-PROTEAN® TGX'M Precast Gels 4-20\% (BioRad) for chromatin fractionation. Electrophoresis was done at $60 \mathrm{~V}$ for $45 \mathrm{~min}$ to allow stacking followed by $120 \mathrm{~V}$ for $1: 30 \mathrm{~h}$ for separation. Proteins were then blotted on nitrocellulose using the TransBlot $^{\circledR}$ Turbo $^{\text {TM }}$ (BioRad) for semi dry blotting and $0.2 \mu \mathrm{m}$ Nitrocellulose Transfer Packs (BioRad) for $30 \mathrm{~min}$ at $25 \mathrm{~V}$ and $1 \mathrm{~A}$. Afterwards, the membrane was removed and stained in Ponceau red $(0.2 \%$ PonceauS, $3 \% \mathrm{AcCH} 3)$ for $5 \mathrm{~min}$ to visualise transferred protein. The membrane was then cut as required for multiple immune staining. Membranes were then washed 5 min in TBS-T (136.89 mM NaCl, 2.68mM, $23.11 \mathrm{mM}$ Tris base, $0.1 \%$ Tween, $\mathrm{pH}$ 7.4) and blocked in $5 \%$ BSA (Roth) TBS-T (0.2 $\mu \mathrm{m}$ filtered, Millex syringe filter, Millipore). After blocking, membranes were incubated with primary antibody (AnxA2, abcam 1:500; Gapdh 1:1000; Actin, Novus 1:1000; H3, abcam 1:1000) in $5 \%$ BSA in TBS-T O/N. Following, membranes were 
washed three times in TBS-T, stained with secondary antibodies IRDye 800CW Goat antiRabbit or IRDye 680RD Goat anti-Mouse $(1: 10000)$ for $1 \mathrm{~h}$ at RT. Afterwards membranes were washed twice in TBST before scanning on a Li-Cor Odyssey infrared fluorescence scanner. Quantification was done with Li-Cor Image studio Lite using median background subtraction, normalised to loading control in Microsoft Excel 2011, plotted and analysed in GraphPad using two-tailed t-test.

\subsubsection{RNA isolation}

For qPCR and next generation sequencing of cultured cells RNA was isolated using TRIzol (Invitrogen) according to vendors instructions with several alterations. In brief, cells were washed once after medium was removed and lysed in one volume (1xV) TRI Reagent (100 $\mu \mathrm{I}$ per 24 well dish, $200 \mu$ l per 12 well dish, $400 \mu$ l per 6 well dish) while thorough vortexing. Alternatively brain samples were homogenised in 1xV TRI Reagent (100 $\mu \mathrm{l} \mathrm{ACC,} 200 \mu \mathrm{l} \mathrm{DG}$, or $500 \mu \mathrm{l} \mathrm{CA1}, \mathrm{CA} 3, \mathrm{Ctx}, \mathrm{Cb}$ ) in a BeadRuptor 24 (Onmi International), subsequently another 1xV was added. After lysis, samples were incubated for $5 \mathrm{~min}$ on $\mathrm{RT}$ before $0.2 \mathrm{xV}$ chloroform were added. Samples were vortexed thoroughly for $15 \mathrm{~s}$ and incubated at RT for $15 \mathrm{~min}$ before centrifugation at $12000 \mathrm{~g}$ for $15 \mathrm{~min}$ at $4{ }^{\circ} \mathrm{C}$. After centrifugation the aqueous phase containing the RNA was transferred into a fresh tube without any carryover of other phases. Now $0.5 \times \mathrm{V}$ Isopropanol was added per sample, vortexed and incubated o/n at $4{ }^{\circ} \mathrm{C}$. On the next day samples were centrifuged at $12000 \mathrm{~g}$ for $30 \mathrm{~min}$ at $4{ }^{\circ} \mathrm{C}$, the supernatant was discarded and the resulting RNA pellet washed twice in $1 \times \mathrm{VV} 75 \% \mathrm{EtOH}$ and subsequently dried for 5 min at RT. Finally, RNA was resuspended in 30-50 $\mu$ PCR grade $\mathrm{H} 2 \mathrm{O}$ (Sigma).

For next generation sequencing from tissue RNA was isolated with NucleoSpin ${ }^{\circledR}$ TriPrep Kit (Macherey-Nagel) according to manufacturer's instructions. Afterwards, RNA concentration was measured in NanoDrop 2000 (Thermo Scientific) and integrity checked using Bioanalyzer (Agilent) "Nano" lab-on-a-chip according to manufacturer's instructions.

\subsection{7. cDNA generation}

cDNA was generated using Transcriptor First Strand cDNA Synthesis Kit (Roche) according to manufacturer's instructions. In brief reagents were composed as indicated in table 3.6 and incubated according to table 3.7 .

3.5.8.

\subsubsection{Quantitative real time $P C R$}

To quantify specific mRNA levels quantitative real time PCR (qPCR) was used. Primers were either designed using UPL Assay Design Centre (Roche) (https://lifescience.roche.com/en de/ brands/universal-probe-library.html) or for Sybr Green assays custom designed as described in "3.5.1. in-silico oligo nucleotide design". Samples were supplemented with primers and reagents 
according to table 3.8 and 3.10 and measured in a LightCycler480 (Roche) with settings according to table 3.9 and 3.11 .

Table 3.8: Sybr Green qPCR protocol

\begin{tabular}{|c|c|c|}
\hline Step & $\begin{array}{c}\text { Temperature } \\
\left.\mathbf{(}{ }^{\circ} \mathbf{C}\right)\end{array}$ & Time \\
\hline Denaturing & 95 & $5 \mathrm{~min}$ \\
\hline $\begin{array}{c}\text { Amplificatio } \\
\text { n (x45) }\end{array}$ & 95 & $10 \mathrm{~s}$ \\
\hline Melting & 65 & $15 \mathrm{~s}$ \\
\hline & 95 & $10 \mathrm{~s}$ \\
\hline & 67 & $15 \mathrm{~s}$ \\
\hline Cooling & 95 & Acquisitio \\
\hline
\end{tabular}

Table 3.10: UPL Master Probe qPCR protocol

\begin{tabular}{|c|c|c|}
\hline Step & $\begin{array}{c}\text { Temperature } \\
\left.\mathbf{(}^{\circ} \mathbf{C}\right)\end{array}$ & Time \\
\hline Denaturing & $95^{\circ} \mathrm{C}$ & $5 \mathrm{~min}$ \\
\hline $\begin{array}{c}\text { Amplificatio } \\
\mathrm{n}(\mathrm{x} 45)\end{array}$ & $95^{\circ} \mathrm{C}$ & $10 \mathrm{~s}$ \\
\hline Cooling & $60^{\circ} \mathrm{C}$ & $30 \mathrm{~s}$ \\
\hline & $72^{\circ} \mathrm{C}$ & $1 \mathrm{~s}$ \\
\hline
\end{tabular}

Table 3.6: cDNA synthesis reagents

\begin{tabular}{|l|c|}
\hline \multicolumn{1}{|c|}{ Reagent } & Volume $(\boldsymbol{\mu l})$ \\
\hline Buffer & 4 \\
\hline RNase inhibitor & 0.5 \\
\hline dNTP & 2 \\
\hline Reverse transcriptase & 0.5 \\
\hline $\begin{array}{l}\text { Random hexamer } \\
\text { primers }\end{array}$ & 2 \\
\hline RNA $(1 \mu \mathrm{g})$ & 11 \\
\hline
\end{tabular}

Table 3.9: Sybr Green reagent composition

\begin{tabular}{|c|c|}
\hline Reagents & $\begin{array}{c}\text { Volume } \\
(\mu \mathrm{l})\end{array}$ \\
\hline PCR- $\mathrm{H}_{2} \mathrm{O}$ & 1.9 \\
\hline Primer F (1:10) & 0.3 \\
\hline Primer R (1:10) & 0.3 \\
\hline SYBR Green MM & 7.50 \\
\hline cDNA (20ng) & 5 \\
\hline
\end{tabular}

Table 3.11: UPL reagent composition

\begin{tabular}{|l|c|}
\hline \multicolumn{1}{|c|}{ Reagents } & $\begin{array}{c}\text { Volume } \\
(\boldsymbol{\mu l})\end{array}$ \\
\hline PCR- $\mathrm{H}_{2} \mathrm{O}$ & 6.05 \\
\hline Primer F (1:10) & 0.15 \\
\hline Primer R (1:10) & 0.15 \\
\hline LC 480 MM & 7.50 \\
\hline $\begin{array}{l}\text { UPL Probe (10 } \\
\mu \text { M) }\end{array}$ & 0.15 \\
\hline cDNA & 1 \\
\hline
\end{tabular}

Table 3.7: cDNA synthesis incubation

\begin{tabular}{|c|c|}
\hline $\begin{array}{c}\text { Time } \\
(\mathbf{m i n})\end{array}$ & Temperature $\left({ }^{\circ} \mathbf{C}\right)$ \\
\hline 10 & 25 \\
\hline 30 & 55 \\
\hline 5 & 85 \\
\hline
\end{tabular}




\subsubsection{RNA sequencing}

To perform whole transcriptome analysis single-end RNA sequencing was performed from previously extracted RNA. Single- end libraries were prepared from $500 \mathrm{ng}$ total RNA using the TruSeq RNA Library Preparation Kit v2 according to manufacturer's instructions. Library quality was validated using the Agilent 2100 Bioanalyzer (Agilent Technologies) using a RNA 6000 Nano Kit, Nanodrop 8000 (Thermo Fisher), Qubit 2.0 fluorometer (Life technologies). Afterwards, the flow cell was clustered using TruSeq SR Cluster Kit v3-cBot-HS following Illuminas standard protocol.

\subsubsection{Single cell sequencing}

Single cell sequencing was performed by Massively Parallel Single-Cell RNA-Seq for MarkerFree Decomposition of Tissues into Cell Types "MARSeq" according to Jaintin and colleagues with the exception of using 6 bases RMTs (Jaitin, Kenigsberg et al. 2014). Sequencing was conducted at DZNE core "Platform for Single Cell Genomics and Epigenomics". In brief, after FACSorting and cell lysis RNA was reverse transcribed during which RMT and UMI-barcodes were added to the RNA polyA-tail. Subsequently transcripts were treated with Exonuclease I to remove nucleotides from single-stranded DNA, then samples were pooled and the second strand synthesised, after which samples were in vitro transcribed and treated with DNAse. RNA was then fragmented and library constructed.

\subsubsection{RNAs sequencing data analysis}

For processing of sequencing data, a customised in-house software pipeline was used. Illuminas bcl2fastq 1.8.4 was used to convert base calls from BCL to FASTQ. Along with base calling, adapter trimming, removal of Unique Molecular Identifiers (UMI) and demultiplexing were performed. Quality control of raw sequencing data was performed using FastQC 0.11.5 (https://www.bioinformatics.babraham.ac.uk/projects/fastqc/). Reads were then mapped using STAR aligner 2.5.2b (Dobin, Davis et al. 2013) to the mouse transcriptome (Mus_musculus.GRCm38.86). Unmapped reads were re-mapped to the mouse genome (mm10). Default settings were used except mismatches were not allowed for the reads $<19 \mathrm{~b}$, reads between $20 \mathrm{~b}$ to $39 \mathrm{~b}$ were allowed one mismatch, incrementing one mismatch for every additional 20b. Read counting on genes or exons was done using HTSeq 0.9.1 (htseq-count, intersection-non-empty) (HTSeq, http://www-huber.embl.de/users/anders/HTSeq) .

\subsubsection{Differential gene expression analysis}

To identify differentially expressed genes, identification and removal of unwanted variation (RUVs) was performed using RUVSeq 1.8.0 (Risso, Ngai et al. 2014). Then DESeq2 1.14.1 (Anders, Reyes et al. 2012) was used for differential expression analysis. Transcripts were 
considered differentially expressed with an adjusted (Benjamini-Hochberg) $p$-value $<0.05$ and basemean $\geqq 25$.

For functional analysis an additional fold-change cutoff was applied for in vivo data at $\log 2 \mathrm{FC} \pm 0.2$ and for in vitro data log2FC \pm 0.5 . Venny (http://bioinfogp.cnb.csic.es/tools/venny/) was used to calculate gene set overlaps. WebGestalt (http://bioinfo.vanderbilt.edu/webgestalt/, (Zhang, Kirov et al. 2005)) was used for Gene Ontology (GO) functional analysis with categories being significantly enriched with $p<0.05$ and false discovery rate $(F D R)<0.05$ if not otherwise indicated. For relative expression (normalised reads per million, NPM) total normalised reads per sample were summed and divided by 1,000,000 which resulted in the scaling factor. Normalised read counts from gene of interest were then multiplied by scaling factor and divided by gene length. For illustration purposes values were additionally multiplied by 100 .

\subsubsection{Gene Set enrichment analysis}

Gene set enrichment was analysed using GSEA (Mootha, Lindgren et al. 2003, Subramanian, Tamayo et al. 2005). Normalised counts from DESeq2 were converted to GCT files then ranked and analysed with following settings, Chip platform: gseaftp.broadinstitute.org://pub/gsea/ annotations/GENE_SYMBOL.chip; number of permutations: 1000; permutation type: phenotype (in vivo) or GeneSet (in vitro).

\subsubsection{Differential exon usage}

Identification of differentially expressed exons was done using DEXSeq 1.22 (Anders, Reyes et al. 2012) according to authors instructions using mm10 reference genome and exon counts from in-house pipeline. Functional analysis was performed for uniquely identified exons with an adjusted $p$-value $<0.05$ and log2FC \pm 0.2 . Venny (http://bioinfogp.cnb.csic.es/tools/venny/) was used to calculate gene set overlaps. Cytoscape 3.0 plugin ClueGO 2.3.3 (Bindea, Mlecnik et al. 2009) (Bonferroni adjusted $\mathrm{p}$-value $<0.05, \mathrm{k}$-score $=0.4$ ) $\mathrm{GO}$ term fusion was used for gene network visualisation using GO ontology for molecular function, biological process and cellular compartment if not otherwise indicated. 
$\frac{0}{5}$

a) 


\section{Results}

\subsection{Annexin A2 is a reader of acetylated $\mathrm{H} 4 \mathrm{~K} 12$ and impacts spatial memory}

\subsubsection{Annexin A2 is a reader of H4K12ac and is ubiquitously expressed in brain regions associated with learning and memory}

Peleg and colleagues previously reported that reduced acetylation of histone 4 at lysine 12 (H4K12ac) correlates with age-associated cognitive impairment (Peleg, Sananbenesi et al. 2010). The acetylation level is orchestrated by HATs (writers) and HDACs (erasers) that add and remove acetyl residues respectively. To interpret the code emerging from these modifications, specific proteins, so called chromatin readers, are required. These proteins recognise specific patterns at the histone tail and subsequently initiate corresponding changes in cellular function. Thus, I investigated by which mechanisms acetylation of H4K12 can affect the chromatin state and in turn the transcriptional outcome. I assumed that the most relevant targets to convey the histone modification into cellular function would be proteins selectively associating with the acetylated version of H4K12 but not with the unmodified. In an unbiased shotgun approach I used stable isotope labelling with amino acids in cell culture (SILAC) to label dissociated primary neuronal cultures, as first published by Spellmann and colleagues (Spellman, Deinhardt et al. 2008). In brief, this technique uses media with amino acids (AA) of higher molecular weight to label proteins in culture. One of the cultures is exposed to conventional (light) AA, while the other is exposed to stable isotopes (heavy), thus making it possible to quantify differential binding of proteins to a given bait. We pulled down soluble nuclear proteins from the cell lysate using a H4K12 or H4K12ac mimic as baits. This allowed us to selectively capture proteins with a high affinity to the acetylation mark associated with the H4K12ac bait but not H4K12. To be able to quantify the affinity of the peptides to the chromatin mark, we performed an experiment in which the acetylated bait was incubated with heavy isotope-labelled amino acids and the unmodified bait was incubated with the light-labelled amino acids (forward experiment). In the complementary experiment, we incubated peptides and baits vice versa (reverse experiment), to assure there is no bias for heavy labelled proteins. To identify chromatin reader specifically binding H4K12ac, we performed LC-MS/MS (Fig. 4.1A). High isotope incorporation is crucial for the success of the experiment, however mature neurons are post-mitotic and thus protein turnover is low. To achieve high incorporation rate we changed $30 \%$ of medium every other day which resulted in $93,1 \%$ labelling efficiency (Fig. 4.1B). LC-MS/ MS revealed 4 peptides significantly associated with the acetylated version of the H4K12 bait but not with the unmodified version (log2 ratio forward $<2$, log2 ratio reverse $<2$ ) (courtesy of Nadin Zimmermann, MPI-bpc, working group chromatin-biochemistry). One of these peptides significantly binding acetylated H4K12 belonged to BRD4, a member of the bromodomain-and extra terminal (BET) protein family. It is a validated H4K12ac reader in immortal cell lines 


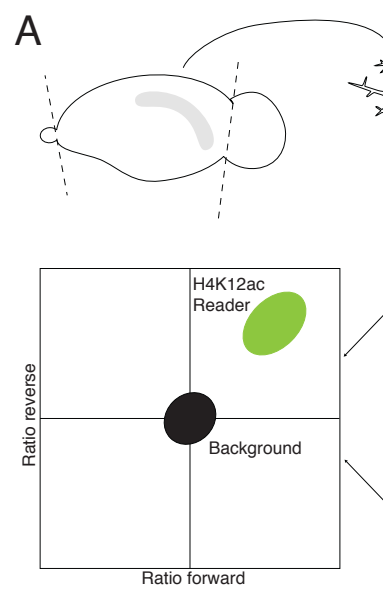

C

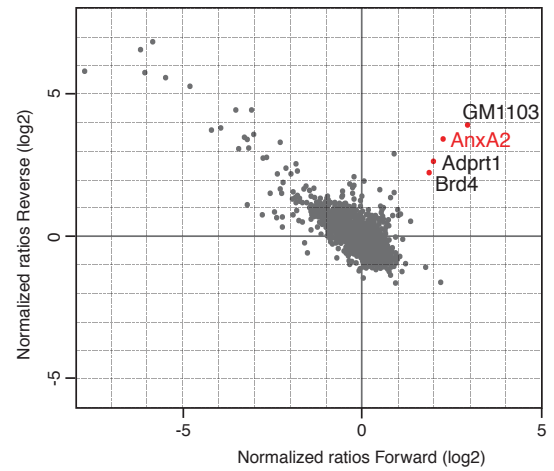

F
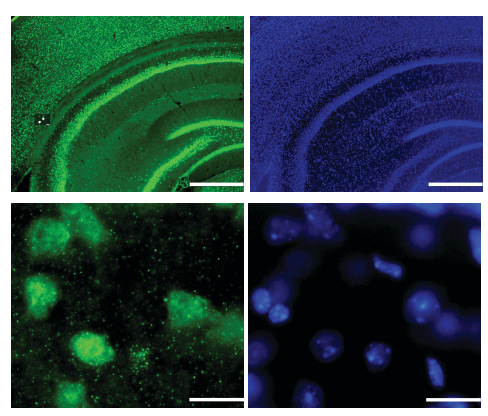
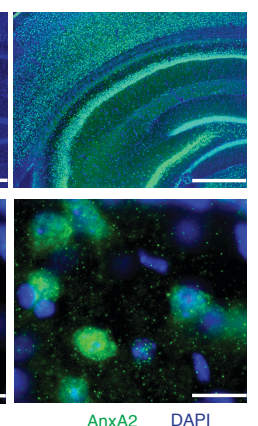

D

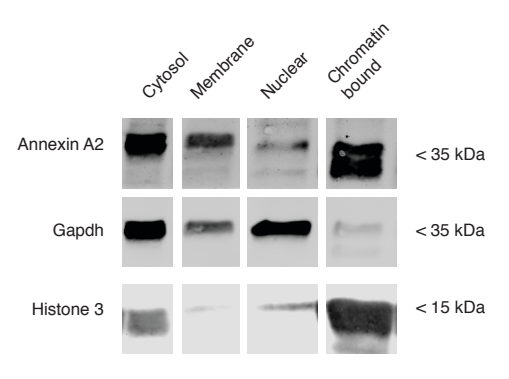

G

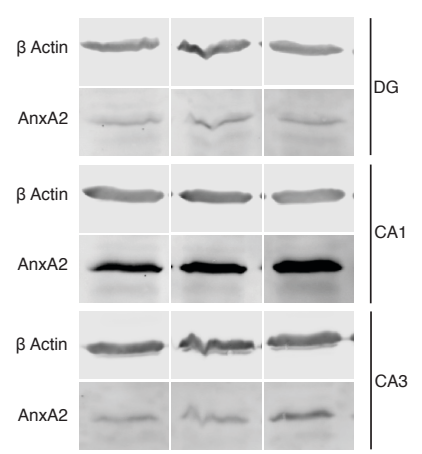

B

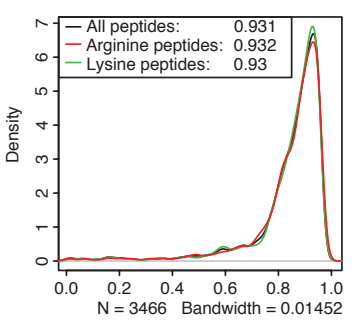

E

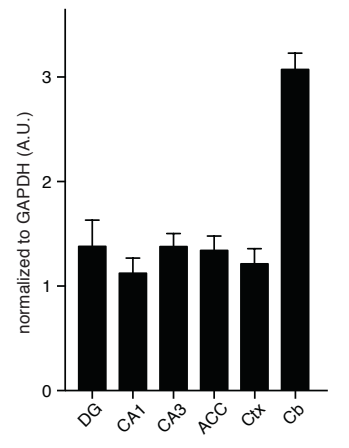

$\mathrm{H}$

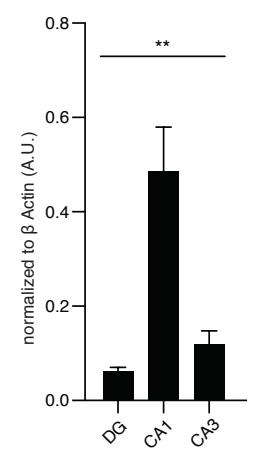

Figure 4.1: ANXA2 is expressed the brain and binds selectively acetylated H4K12ac. Illustration of the preparation of heavy and light amino acid labelled primary neuronal culture with subsequent chromatin purification and forward and reverse peptide pulldown using H4K12ac or H4K12 bait. Peptides were quantified based on mass differences introduced by heavy isotope labelling using LC-MS/MS. Green area indicates range of expected chromatin readers (A). Peptides were efficiently labeled with heavy Arginine and Lysine isotopes at $93.1 \%$ of total peptides in heavy samples (B). LC-MS/MS identified 4 readers specific for $\mathrm{H} 4 \mathrm{~K} 12 \mathrm{ac}$ but not $\mathrm{H} 4 \mathrm{~K} 12$ (log2 ratio forward<2, log2 ratio reverse $<2$, padj $<0.05$ ) enrichment ratio with ANXA2 being the most significant of the at the time annotated (C). Western blot showed ANXA2 was present across cell fractions and highly abundant in chromatin fraction (D). AnxA2 mRNA was present in dentate gyrus (DG), cornus ammonis (CA1, CA3), anterior cingulate cortex (ACC), cortex (Ctx) and cerebellum (Cb) ( $q P C R, n=4)(E)$. ANXA2 (green) protein can be detected by immunohistochemistry across brain regions, including hippocampus and cortical layers (bar $=500 \mu \mathrm{M}$ ). ANXA2 co-localises with DAPI as shown for DG (blue) (bottom, bar $=25 \mu \mathrm{M})(\mathrm{F})$. Western blot showed ANXA2 was present in DG, CA1, and $C A 3(G)$ and significantly increased in CA1 when quantified and normalised to $\beta$-Actin $(p=0.0320$, unpaired two tailed t-test, $n=4)(H)$. Error bars indicate $S E M,{ }^{*}=p<0.05$, ${ }^{* *}=p<0.001,{ }^{* *}=p<0.0001$. (A) was conducted with Dr. Eva Benito, $(B-C)$ are courtesy of Nadin Zimmermann. 
(Filippakopoulos and Knapp 2012, Jung, Philpott et al. 2014), confirming our approach. Other identified readers in ascending affinity for the H4K12ac bait were: ADPRT1 (PARP1), a poly(ADP-ribosyl)transferase; ANXA2, a calcium-dependent phospholipid-binding protein; and GM1103 (at the time not annotated, now ZBTB2), presumably involved in transcriptional regulation (Jeon, Choi et al. 2009, Kim, Koh et al. 2015) (Fig. 4.1C). Notably, in neuronal lysates these readers showed higher affinity to the H4K12ac bait than the previously reported BRD4 which could indicate a higher selectivity for the mark.

To study the role of neuronal $\mathrm{H} 4 \mathrm{~K} 12 \mathrm{ac}$ readers in greater detail and to understand how this modification changes the epigenetic landscape, I decided to focus on ANXA2. It showed the highest affinity to H4K12ac amongst the annotated readers and had been reported to interact with chromatin (Das, Shetty et al. 2010, Oh, Gao et al. 2013). I hypothesised that a reader of a chromatin mark that is relevant to learning and memory would in turn itself be important for cognitive performance. Thus I focused on neuronal tissue and started to characterise the subcellular localisation of ANXA2 in the cortex of 3 month old mice, using Western blot. ANXA2 was detected as a robust band at approximately $36 \mathrm{kDa}$ and located in all cellular compartments tested, namely cytosol, membrane, and nucleus. As it corresponds to chromatin readers, AnxA2 was particularly strong in the chromatin-bound fraction (Fig. 4.1D). Further, I tested AnxA2 mRNA expression by qPCR in brain regions associated with learning and memory, as well as in the cerebellum for comparison. This revealed the strongest expression in cerebellum $(\mathrm{Cb})$ and $\mathrm{a}$ robust and comparable expression of AnxA2 mRNA across other subregions, namely dentate gyrus (DG), cornus ammonis (CA1, CA3), anterior cingulate cortex (ACC) and cortex (Ctx) (Fig. 4.1E). Subsequently, I tested where ANXA2 was present at the protein level across different brain regions. Immunohistochemistry showed robust expression of ANXA2 throughout the hippocampus including the DG, CA1, CA3 and across cortical layers. DAPI co-localisation indicated nuclear localisation in 3 month old mice (Fig. 4.1F). Since the quantitative resolution in immunohistochemistry is restricted, I additionally performed Western blot analysis for hippocampal subregions. Here too, ANXA2 was present throughout the DG, CA1, and CA3 of 3 month old mice (Fig. 4.1G). ANXA2 protein levels were significantly increased in CA1 compared to other subregions ( $p=0.032$, unpaired two tailed t-test) (Fig. 4.1H). Thus, we identified AnxA2 as a neuronal reader of $\mathrm{H} 4 \mathrm{~K} 12 \mathrm{ac}$ in an unbiased screen and hypothesised that it might be involved in cognitive function. I characterised its expression at the mRNA and protein level and found it to be expressed in the cortical and particularly in hippocampal subregions. These results were encouraging to continue the characterisation of ANXA2 in the aged mouse to asses whether the reported age-dependent changes in acetylation of $\mathrm{H} 4 \mathrm{~K} 12$ translate into alterations of its reader.

\subsubsection{Translation and localisation of ANXA2 change in ageing}

As mentioned above, Peleg and colleagues showed that acetylation of H4K12 was less abundant in aged mice and correlated with their cognitive decline (Peleg, Sananbenesi et al. 
2010). Thus, I investigated whether ANXA2, as a reader of H4K12ac, would change in expression or localisation during ageing. To investigate this, I compared mRNA levels in 3 and 16 month old animals using qPCR. I learning and memory associated brain regions (DG, CA1, $\mathrm{CA} 3, \mathrm{ACC}, \mathrm{Ctx}$ ) and $\mathrm{Cb}$. However, I found no significant differences between the groups (Fig. 4.2A). I also analysed protein levels in hippocampal subregions DG, CA1, CA3 using Western blot and found that ANXA2 was present throughout the regions in 16 month old animals (Fig. 4.2B). In contrast to 3 month old mice, no difference between hippocampal subregions was detected. However, comparing protein levels between the young and old cohort revealed significantly lower ANXA2 levels in CA1 of aged mice ( $p=0.032$, unpaired two tailed ttest) (Fig. 4.2C). Since I previously observed a nuclear localisation of ANXA2 in the hippocampus of 3 month old mice, I performed immunohistochemistry on sagittal sections of both, 3 month and 16 month old animals to compare both cohorts. This revealed an increase of nuclear ANXA2 signal in aged mice compared to young controls (Fig. 4.2D). Fluorescence

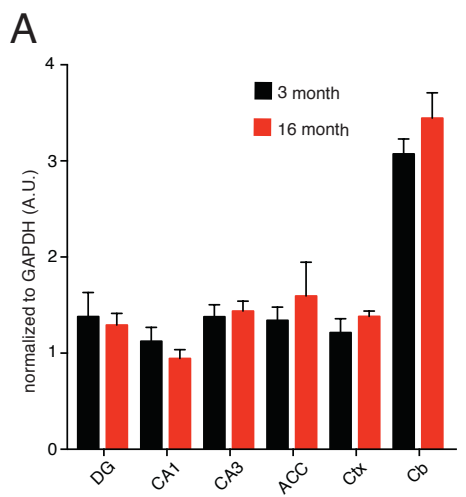

D

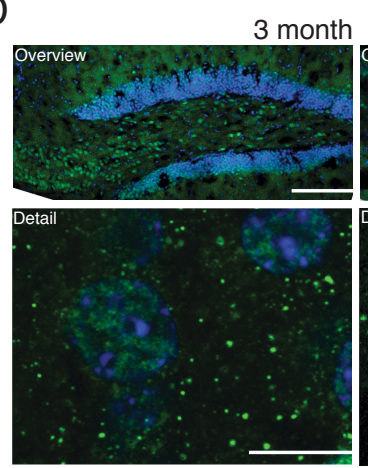

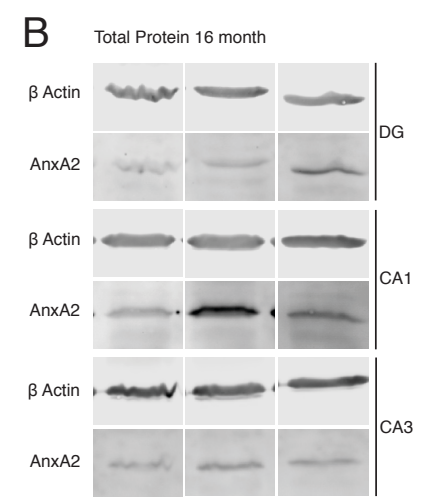

E

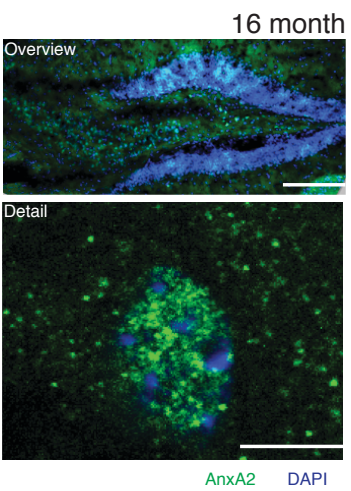

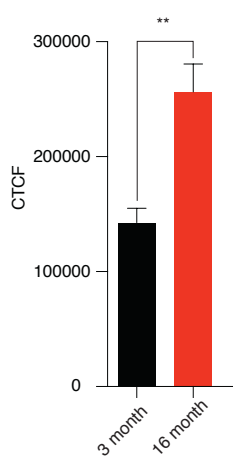

C

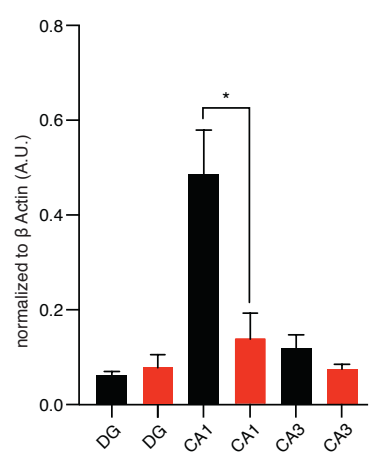

F

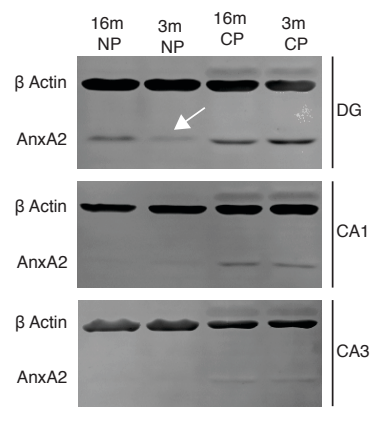

Figure 4.2: Protein levels of ANXA2 are reduced in CA1 and increased in nuclei of DG in aged mice. AnxA2 mRNA was not changed in dentate gyrus (DG), cornus ammonis (CA1, CA3), anterior cingulate cortex (ACC), cortex (Ctx) and cerebellum (Cb) between 3 and 16 months of age (qPCR, $n=4)$ (A). Protein levels of ANXA2 were similar in DG, CA1, and CA3 of 16 month old mice $(n=4)(B)$. Quantification of $(B)$ showed significantly decreased protein levels in CA1 of 16 month old WT mice compared to the 3 month old control $(p=0.032$, t-test, $n=4)(C)$. ANXA2 was expressed in the hippocampus of 3 and 16 month old mice scale bar $=250 \mu \mathrm{M}$ (D, top). In the DG nuclear ANXA2 was increased in the aged group compared to young as revealed by immunohistochemistry on sagittal sections scale bar=10 $\mu \mathrm{M}(\mathrm{D}$, bottom). CTCF intensity quantifications from (D) $(p=0.0003$, t-test, $n=16)$ (E). Western blot analysis of sub cellular fractionations for nuclear protein (NP) and cytosolic protein (CP) of DG, CA1, CA3 of 3 month versus 16 month old mice arrow indicates difference between 16 and 3 month old mice (F). Error bars indicate SEM, ${ }^{*}=p<0.05,{ }^{\star \star}=p<0.001,{ }^{* \star *}=p<0.0001$. 
intensity quantifications (CTCF) of the immuno-staining indicated the difference to be significant $(p=0.0003$, t-test, $n=16$ ) (Fig. 4.2E). This finding was further supported by Western blot analysis of sub-cellular fractionations of hippocampal regions DG, CA1, CA3 of 3 month versus 16 month old mice. ANXA2 was increased in the nuclear fraction of the DG of aged animals compared to young, while other areas did not show nuclear ANXA2 and comparable cytosolic levels (Fig. 4.2F). ANXA2 did not show an alteration in localisation in CA1 and CA3 when comparing 3 month and 16 month old animals.

So far, the data indicate that AnxA2 is a neuronal chromatin reader with high affinity for $\mathrm{H} 4 \mathrm{~K} 12 \mathrm{ac}$. It is robustly expressed in learning and memory related areas and particularly in the hippocampal subregions. Most interestingly, there seems to be a relation between ANXA2 subcellular localisation and raging, with AnxA2 accumulating in the nucleus in 16 month old animals compared to 3 month old animals. Bases on this evidence, there are two probable explanations: the increase of AnxA2 levels in the nucleus in DG of aged animals and the decrease in CA1 could either counteract cognitive decline or be a detrimental phenotype in ageing. In order to distinguish these possibilities and to establish whether there is a functional relationship between AnxA2 levels and sub-cellular distribution and cognition, I went on to study the influence of increased AnxA2 levels in the hippocampus in a gain of function model.

\subsubsection{AnxA2 overexpression impairs hippocampus-dependent spatial memory} The characterisation of the localisation and expression levels of ANXA2 in the young and aged mouse brain, revealed that ANXA2 was increased in the DG nuclear fraction while also decreased in the CA1 of aged mice. Thus, I designed two sets of complementary experiments to address the role of ANXA2 in learning and memory: firstly, a gain of function approach with AnxA2 overexpression and secondly, AnxA2 knockout.

In order to overexpress AnxA2, I chose adeno-associated viral constructs (AAVs) due to their high specificity and low tendency to spread compared to other viral delivery methods. I produced two AnxA2 and one control construct expressing eGFP. In brief, I cloned murine AnxA2 under the control of the hSyn promoter for neuron-specific expression into the AAV backbone and fused it to eGFP for tracking (hSyn-AnxA2/GFP, "ANX") (Fig. 4.3A). To study the impact of ANXA2 sub-cellular localisation on learning and memory, I produced an alternative version of the vector with a nuclear localisation signal (NLS) fused in frame with eGFP. The backbone with eGFP under the control of the hSyn promoter (hSyn-GFP, "GFP") was used as control (Fig. 4.3A). As expected, NLS addition to the C-terminus of eGFP led to nuclear accumulation of the ANXA2 fusion protein (hSyn-AnxA2/GFP/NLS, "NLS") when compared to ANXA2-GFP in primary neuronal culture (DIV10) (Fig. 4.3B). For behavioural experiments, the AAV particles were injected into the DG of 3 month old mice, which resulted in hippocampus specific and DG-enriched overexpression of the constructs (Fig. 4.3 C-E). After three weeks of viral incubation, I performed a behavioural test battery consisting of anxiety (elevated plus maze 
A

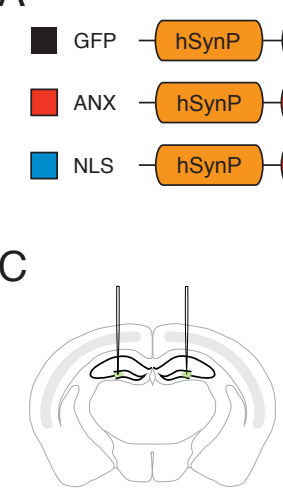

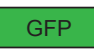
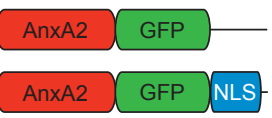

B

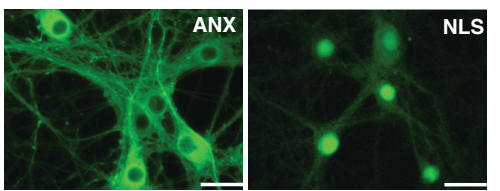

$E$

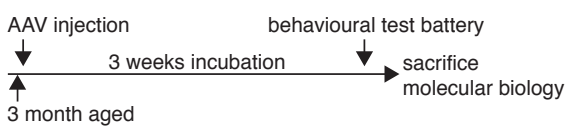

Figure 4.3: Expression of ANXA2 in cytosol and nucleus using AAVs. Three vectors were produced using identical backbones and hSynP for neuronal specificity. The control construct hSynP-GFP only harboured GFP ("GFP, black"). A second construct hSynP-AnxA2-GFP additionally featured information for 339 AA AnxA2 fused to GFP ("ANX, red"). A third construct hSynP-AnxA2-GFP-NLS featured the 339 AA AnxA2 fused GFP with the addition of a NLS at the GFP C-terminal ("NLS, blue"). AAV1/2 hybrids were used for delivery of the constructs (A). ANXA2 was expressed mainly in the cytosol (ANX) or the nucleus (NLS) in primary neuronal cultures (DIV10, 6 days post infection) scale bar=25 $\mu \mathrm{M}$ (B). Schematic coronal illustration of stereotactic intracranial injection of AAVs into DG using micro-capillaries (C). Mouse brain 11 weeks after injection of hSynP-AnxA2-GFP-NLS into the DG of 3 month old male mice. Hippocampus was morphologically unaffected and showed robust transgene expression exclusively in hippocampal regions (D). Schematic of treatment protocol, 3 month old male mice were injected with the respective virus, incubated for 3 weeks with subsequent behavioural test battery and sacrificed prior to molecular biology analysis (E).

"EPM", open field test "OF"), fear memory (contextual fear conditioning "FC") and spatial memory tests (Morris water maze "MWM", and Barnes maze "BM").

ANXA2 overexpression in ANX or NLS did not significantly change the percent of time spent in the open arms of the EPM, indicating comparable anxiety levels across groups (Fig. 4.4A). This finding was further supported by comparable levels of exposure and travel speed between all tested groups in the OF, also indicating motor skill was unaffected (Fig. 4.4B+C). After demonstrating similar levels of anxiety, I performed hippocampus-dependent cognitive tests. In FC, an associative memory task, all groups showed comparable initial freezing during training and significantly increased freezing after the learning stimulus on the successive day $(F(1,78)=130.3, p<0.0001$, two-way ANOVA $)$ with no significant difference between cohorts (Fig. 4.4D).Thus, AnxA2 overexpression had no significant influence on associative fear memory. I performed a second spatial memory test, the MWM, which is more heavily dependent on the hippocampus. Animals were trained for 12 consecutive days during which the latency to find the submerged platform (escape latency) was taken as a measure of learning performance. Probe tests (PT) were performed on days 6,11 , and 13 , when the first cohort reached a plateau at a cumulative escape latency of less than $20 \mathrm{~s}$. ANX and GFP performed similarly during the acquisition ( $p>0.05, F(1,26)=0.11$, two-way-ANOVA), while the NLS cohort showed reduced performance in the MWM compared to $\operatorname{ANX}(F(1,28)=6.34, p=0.018$, two-way-ANOVA $)$ and GFP controls $(F(1,26)=5.67, p=0.025$, two-way-ANOVA) (Fig. 4.4E). However, this observation was not supported by the evaluation of spatial memory on any of the PT (Fig. 4.4F+G), indicating a 
A

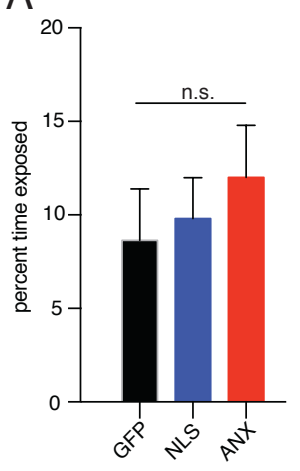

B

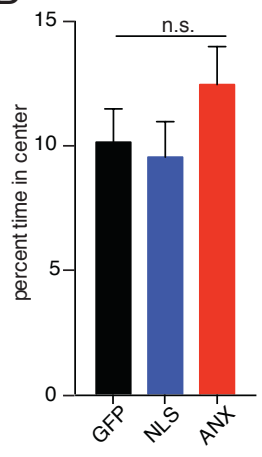

C

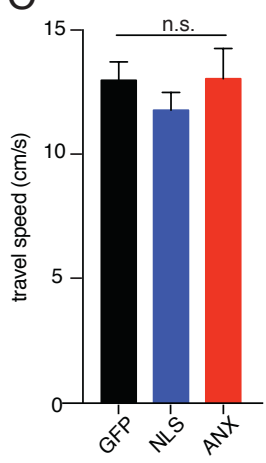

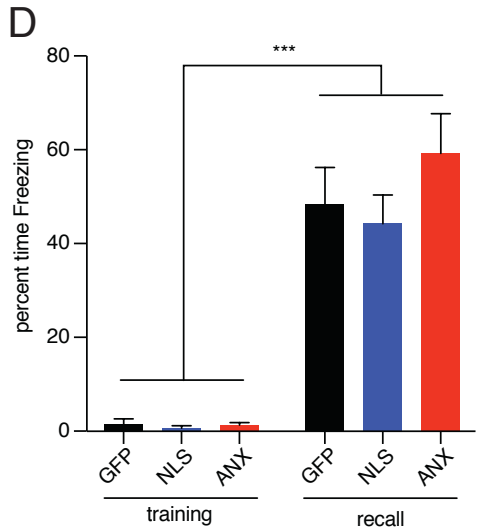

G
$\mathrm{E}$

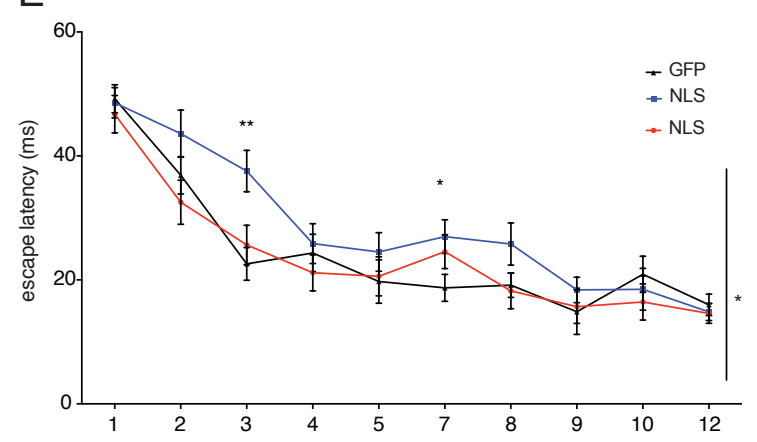

$\mathrm{F}$

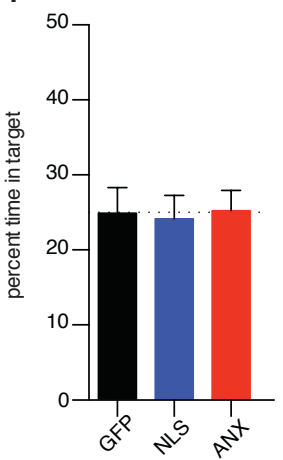

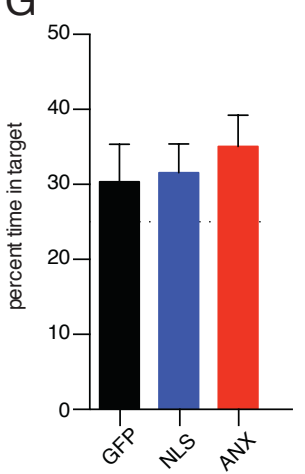
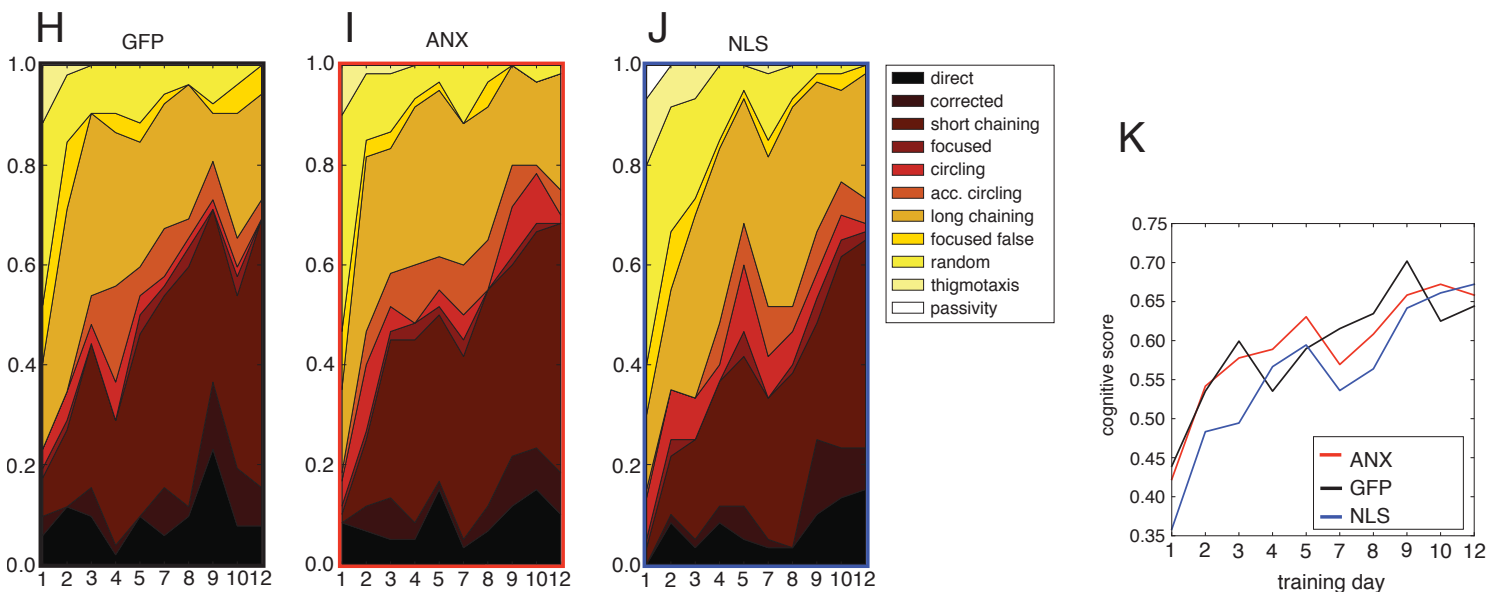

Figure 4.4: Behavioural testing of ANXA2 overexpressing mice shows influence of ANXA2 on cognitive function. Elevated plus maze (EPM) test for anxiety showed similar amount of time spent in open arms as percent of total time for all groups (GFP, black, $n=13$; ANX, red, $n=15$; NLS, blue, $n=15$ ) $(F(2,40)=0.4348, p=0.650)(A)$. Time spent in central area relative to total experimental duration of the open field test (OF) sustained the EPM finding by demonstrating comparable anxiety levels $(F(2,39)=1.175, p=0.320)$ (B) and further showed similar travel speed $(\mathrm{cm} / \mathrm{min})$ in all cohorts $(F(2,40)=0.5874, p=0.561)$ (C). All cohorts displayed similar initial freezing (displayed as percent of total time) in contextual fear conditioning during training and recall. Importantly, cohorts showed a significant increase in freezing after training (recall) $(F(1,78)=130.3, p<0.0001$, two-way ANOVA) (D). Morris water maze revealed ANX and GFP performed similar while NLS exhibited increased escape latency compared to both ANX $(F(1,28)=6.343, p=0.018$, two-way-ANOVA) and GFP controls $(F(1,26)=5.673$, $\mathrm{p}=0.025$, two-way-ANOVA) (E). Performance in probe test on day 6 was similar between groups $(F(2,40)=0.034, p=0.967)(F)$. Probe test on day 13 showed increased time spent in target for all groups without significant difference between groups $(F(2,40)=0.320, p=0.728)(G)$. GFP controls and ANX used cognitively challenging search strategies (direct-focused) $(\mathbf{H}+\mathbf{l})$. NLS tended to use less demanding strategies (circling-thigmotaxis) and passivity (J). Display of cumulative strategy scores from (H-J) indicated NLS use lower cognitive scores compared to ANX and GFP (K). One-way ANOVA was used, if not stated otherwise. Error bars indicate SEM, ${ }^{*}=p<0.05,{ }^{* *}=p<0.001,{ }^{* \star *}=p<0.0001$. 
subtle phenotype. Since the introduced manipulations could affect learning capabilities without affecting memory consolidation, I investigated search strategies in the maze. GFP controls showed a longitudinal increase in strategies with higher cognitive scores (direct-focused search) and in turn a decrease in low cognitive scores (circling-thigmotaxis) (Fig. 4.4H). ANX showed a similar performance with pronounced focused search from training day 3 onwards (Fig. 4.4I). In contrast, NLS used less cognitively challenging strategies (accidental circling-thigmotaxis) and a slow longitudinal increase of higher cognitive strategies (direct-focused), as well as increased passivity (Fig. 4.4J). These findings were also represented in the cumulative scores, were NLS performed poorly compared to other cohorts (Fig. 4.4K).

Behavioural results suggested that overexpression of ANXA2 AnxA2 may play a subtle role in modulating spatial memory performance in the mouse hippocampus. To gain a greater insight into this modulatory function, I tested animals with the Barnes maze, a more subtle subtle spatial and hippocampus-dependent, which is less stressful to the animal and thus reduces noise in the data (Harrison, Hosseini et al. 2009). The paradigm was modified from Attar and colleagues to further reduce stress to the test subjects (Attar, Liu et al. 2013) (Fig. 4.5A). Memory function was scored based on the time spent in the quadrant containing an escape cage hidden below one of 20 holes (target, T) relative to the other quadrants (left, L; opposite, $\mathrm{O}$; right, R) Fig. 4.5B). Importantly, all cohorts travelled comparable distances, ruling out a motivational or motor impairment (Fig. 4.5C). After three days of training a PT was performed, in which the GFP cohort showed significantly increased time spent in the target quadrant as compared to off-target quadrants $(F(3,48)=9.49, p<0.0001$, one-way ANOVA). NLS animals performed similarly to controls and spent significantly longer time in the target quadrant compared to other quadrants $(F(3,55)=5.46, p=0.002$, one-way ANOVA). In contrast, ANX failed to learn the task after 3 days of training, indicated by equal percentages of time in all quadrants $(F(3,49)=0.35, p=0.792$, one-way ANOVA) (Fig. 4.5D). Importantly, this difference was significant also when compared to GFP controls $(F(2,38)=3.43, p=0.043$, one way ANOVA) (Fig. 4.5E). To evaluate if the ANX cohort was able to learn this task, I continued the training for an additional 4 days after 2 days recovery to reduce memory extinction. Also in the second PT all cohorts displayed comparable distance travelled $(F(2,40)=1.07, p=0.353$, one way ANOVA) (Fig. 4.5F). Additional training resulted in longer time spent in the target quadrant in both GFP and NLS cohorts (GFP: $F(3,46)=14.75, p<0.0001$; NLS: $F(3,55)=65.04, p<0.0001$, one-wayANOVA), which also had shown significant preference for the target quadrant. Importantly, ANX too spent significantly more time in the target quadrant compared to other quadrants during the second PT $(F(3,51)=27.41, p<0.0001$, one-way ANOVA) (Fig. 4.5G), indicating that ANX are generally capable of learning the task. Of note, cohorts did not differ in the second probe test $(F(2,39)=0.54, p=0.586$, one-way ANOVA $)$. However, ANX still showed a trend towards less time spent in the target quadrant, when compared directly to other cohorts (Fig. $4.5 \mathrm{H}$ ).

While NLS showed a mild impairment in learning in the MWM, none of the groups deviated significantly from chance level in their time spent in target quadrant. This finding put the 
reliability of the MWM in this line of experiment into question. However, in the BM control animals and NLS show robust learning and memory, while ANX learned the task after additional training. Taken together this data indicates an involvement of AnxA2 in learning and memory.

A

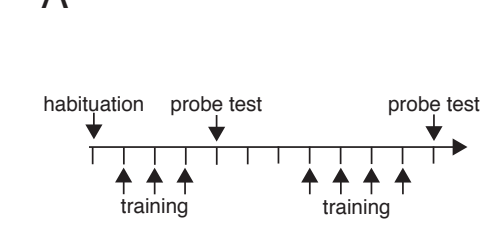

B

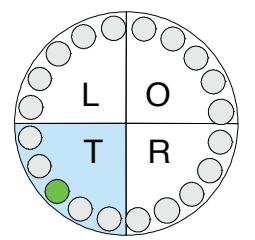

C

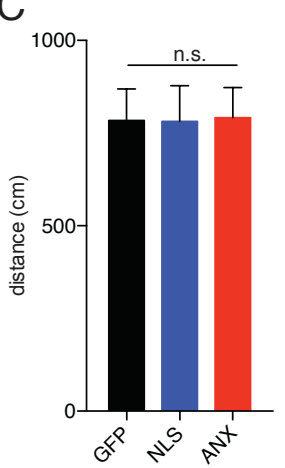

$\mathrm{F}$

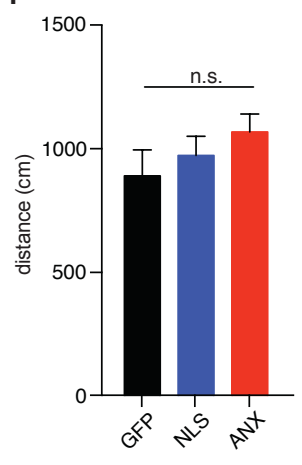

$\mathrm{D}$

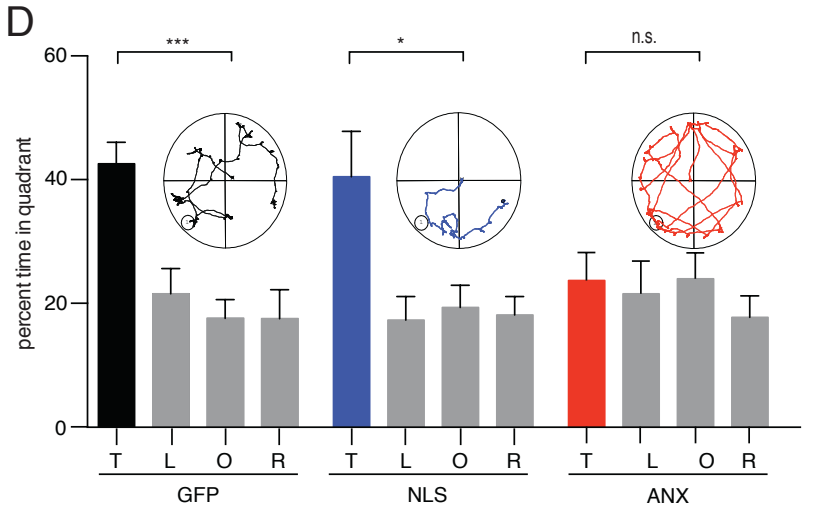

$G$

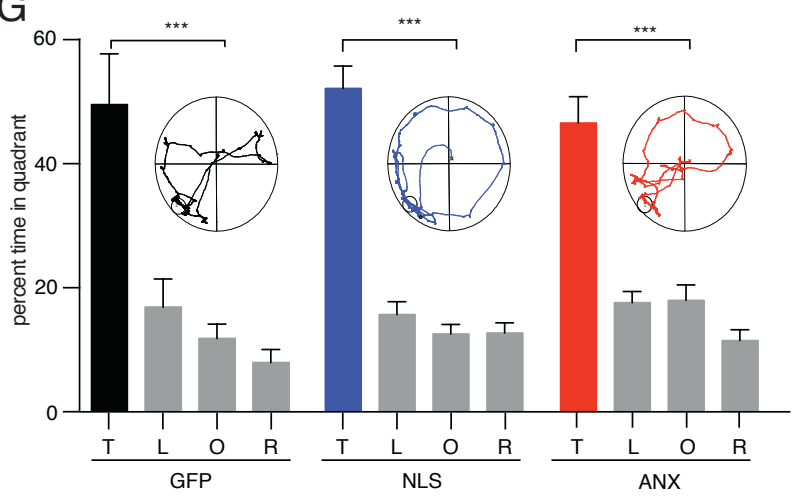

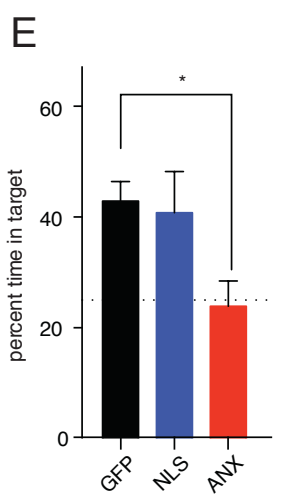

$\mathrm{H}$

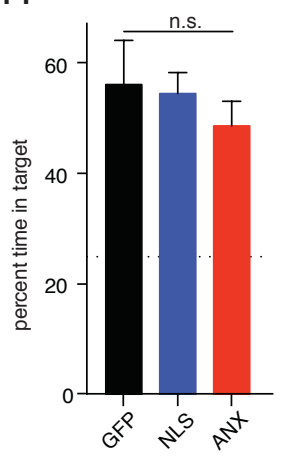

Figure 4.5: Spatial memory is impaired in mice overexpressing ANXA2 in hippocampal DG neurons. Barnes maze (BM) test paradigm for GFP, black, $n=13$; ANX, red, $n=15$; NLS, blue, $n=15$. Animals were habituated for one day followed by 3 consecutive training days with one session each and a subsequent probe test (PT). Animals were allowed to rest for 2 days after PT then trained for additional 4 days and followed by a second PT (A). BM experimental setup consisted of a round white plate $(92 \mathrm{~cm}$ diameter) and 20 holes separated into 4 quadrants. Target quadrant ( $T$, blue) featured an escape cage hidden below the target hole (green), off target quadrants were separated into left (L), opposite (O), and right $(\mathrm{R})$ (B). All cohorts traveled comparable distances $(\mathrm{cm})$ during first $\mathrm{BM}$ probe test $(\mathrm{PT})$ $(F(2,40)=0.004, p=0.996)(C)$. During first PT GFP $(F(3,48)=9.485, p<0.0001)$ and NLS $(F(3,55)=5.46$, $p=0.002)$ spent significantly longer time in $T$. ANX did not show a preference for $T(F(3,49)=0.35$, $p=0.792)(D)$. Direct comparison of percent time spent in T from (D): ANX spent significantly less time in T compared to GFP controls $(F(2,38)=3.43, p=0.043)(E)$. All groups travelled comparable distance $(\mathrm{cm})$ during the second PT $(F(2,40)=1.07, p=0.353)(F)$. Second PT showed significant preference in all cohorts for $T$ (GFP: $F(3,46)=14.75, p<0.0001$; NLS: $F(3,55)=65.04, p<0.0001$; ANX: $F(3,51)=27.41$, $\mathrm{p}<0.0001)(G)$. Direct comparison of percent time spent in $T$ from $(G)$ showed no significant difference between groups $(F(2,39)=0.54, p=0.586)(H)$. One-way ANOVA was used for multiple group comparison, error bars indicate SEM, ${ }^{*}=p<0.05,{ }^{* *}=p<0.001,{ }^{\star \star *}=p<0.0001$. 


\subsubsection{ANXA2 null mutants display facilitated spatial memory}

To further sustain the finding that ANXA2 is involved in correct hippocampus-dependent spatial memory, I studied the influence of ANXA2 in a loss of function model. Considering the accumulation of AnxA2 in the DG of old animals and the impaired spatial memory in mice overexpressing ANXA2, I hypothesised that ANXA2 deficiency would result in the complementary phenotype. To test this, I used ANXA2 knockout (KO) and wild-type (WT) littermates and confirmed the absence of ANXA2 protein in whole brain lysate (Fig. 4.6A). Next, I subjected the mice to a behavioural test battery composed of an anxiety test (OF) and hippocampus-dependent memory tasks for fear memory (FC) and spatial memory (MWM, BM). In the OF test both KO and WT performed similarly with no significant difference between cohorts in both measures; time spent exposed (Fig. 4.6B) and travel speed (Fig. 4.6C). To assess hippocampus-dependent associative memory FC was performed. During training WT and $\mathrm{KO}$ displayed comparable initial freezing behaviour and a minor trend towards increased freezing in KO. Also during recall both groups showed similar freezing behaviour, while both cohorts displayed significantly increased freezing compared to the pre-shock condition $(F(1,20)=48, p<0.0001$, two-way ANOVA) (Fig. 4.6D). Further, I tested the animals in the MWM for spatial memory assessment. PTs were conducted for both groups when one cohort reached an average escape latency of less than $20 \mathrm{~s}$, after 4 and 8 days respectively. KO had overall lower latencies to reach the escape platform compared to WT, indicating facilitated memory acquisition and consolidation $(F(1,70)=7.24, p=0.009$, 2-way ANOVA) (Fig. 4.6E). The first PT indicated a trend in $\mathrm{KO}$ to spend more time in target quadrant as compared to WT (Fig. 4.6F). This persisted, yet less strikingly, throughout the second PT, while WT spent slightly more time in the target quadrant compared to the first PT (Fig. 4.6G). Notably, neither of the groups spent time in the target quadrant above chance level (25\%) during PTs. Since these findings point towards an enhancement in the KO cohort, which can be explained by search strategies rather than target occupancy, I investigated search strategies during the training period for both WT and KO. WT showed fluctuating usage of high cognitive strategies (direct-focused search) during all 8 days of training (Fig. $4.6 \mathrm{H}$ ). In contrast, KO showed an increase of high cognitive strategies from day 3 on and they sustained these strategies throughout the experiment (Fig. 4.6I). These findings were further confirmed by the cumulative cognitive scores per day, here, KO outperformed WT from training day 3 onward (Fig. 4.6J).

Subsequently, animals were tested in the more sensitive BM to detect more subtle changes, as previously exemplified. The test was conducted for WT and KO as previously described for the overexpression experiments (Fig. 4.7A), where time spent in the quadrant $(T)$ formerly containing the hole to the escape cage was compared to other quadrants (left, L; opposite, O; right, R) as a proxy for spatial memory (Fig, 4.7B). During the first probe test both WT and KO travelled equal distances, indicating equal motivation and motility (Fig. 4.7C). During the first PT WT did not spent more time in target compared to off-target quadrants $(F(3,16)=1.76, p=0.196$, one-way ANOVA), while KO animals showed enhanced performance and spent significantly 
A

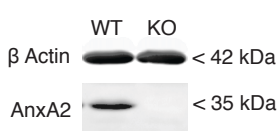

$\mathrm{E}$

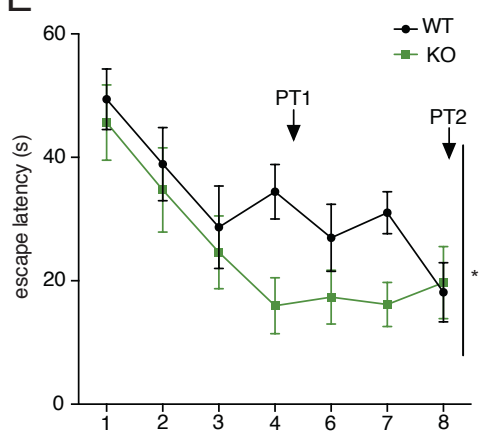

C

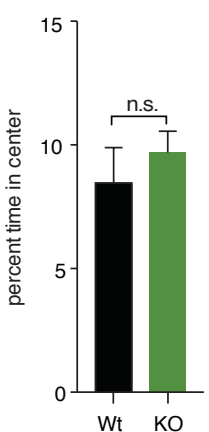

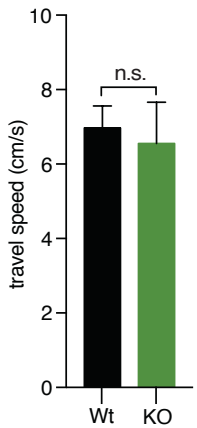

$\mathrm{D}$

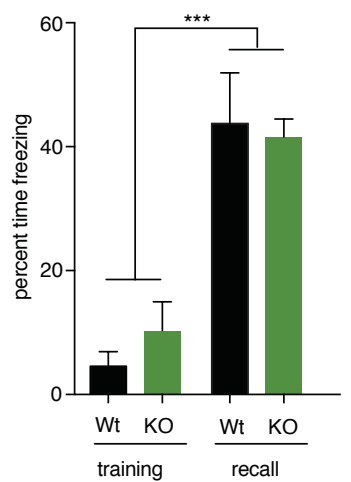

$\mathrm{F}$

G
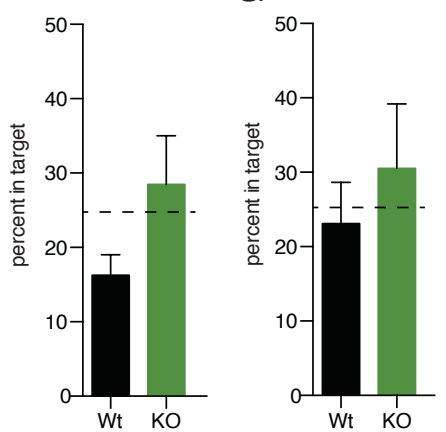

$\mathrm{H}$

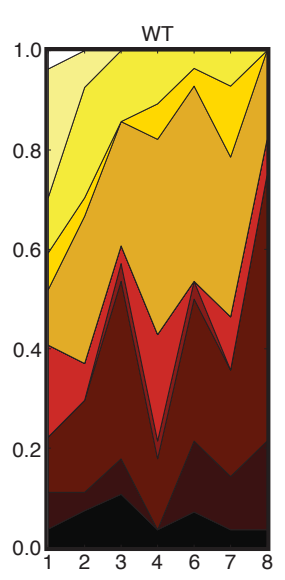

1
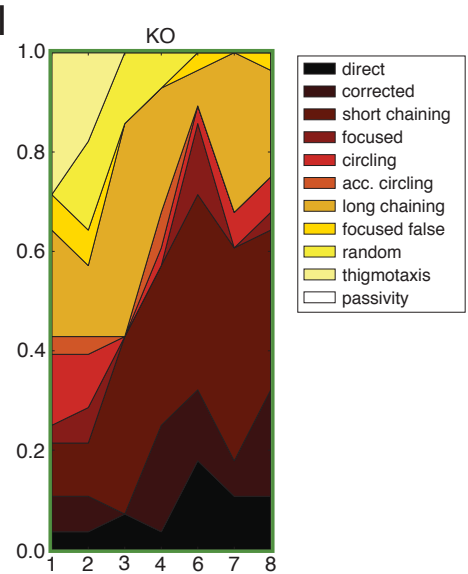

$J$

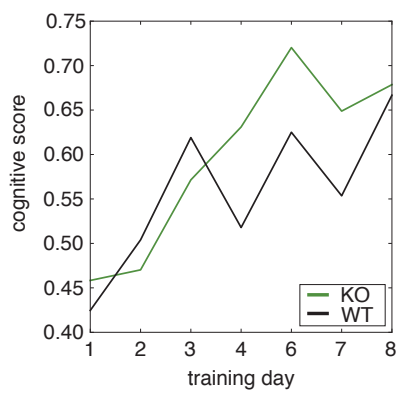

Figure 4.6: ANXA2 null mutants show enhanced performance in spatial memory. ANXA2 protein was not detectable in total brain lysates as demonstrated by Western blot (A). Open field (OF) showed equal length of time spent in the centre for both wild-type (WT, black, $\mathrm{n}=7)$ and $A N X A 2^{-1-}$ mice (KO, green, $\mathrm{n}=7)(\mathrm{p}=0.495$, unpaired t-test $(\mathrm{B})$. OF did not show differences in travel speed $(\mathrm{p}=0.759$, unpaired $\mathrm{t}$-test $)$ (C). WT and KO showed comparable initial freezing in training ( $\mathrm{p}=0.297$, unpaired $\mathrm{t}$-test) and recall $(\mathrm{p}=0.812$, unpaired t-test) but significant increase in percent of total time freezing when comparing training and recall $(F(1,20)=48, p<0.0001$, two-way ANOVA) (D). During MWM training KO showed significantly shorter escape latencies compared to $W T(F(1,70)=7.242, p=0.009$, two way ANOVA) (E). probe tests (PT1, PT2) were performed when cohorts reached average escape latency $<20 \mathrm{~s}$. Percent of time spent in target during PT1 showed a non-significant tendency of KO to spend more time in T compared to WT $(p=0.118$, unpaired t-test) $(\mathbf{F})$. Percent of time spent in the target quadrant during PT2 too showed non-significant tendency of KO to spent more time in T compared to WT ( $p=0.485)(G)$. In MWM WT showed fluctuating usage of high cognitive strategies (direct-focused search) during MWM training $(\mathbf{H})$. KO showed increased use of higher cognitive strategies (I). Cumulative cognitive scores per day indicated KO outperformed WT from training day 3 onward $(\mathbf{J})$. Error bars indicate SEM, ${ }^{*}=p<0.05$, ${ }^{* *}=p<0.001,{ }^{* \star *}=p<0.0001$. 
A

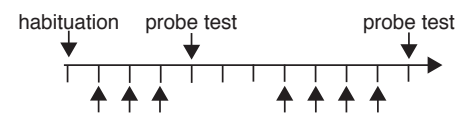

B

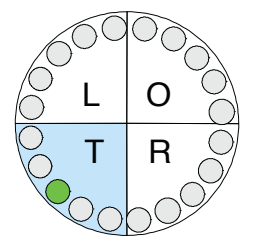

C

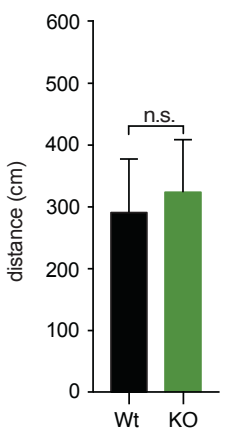

F

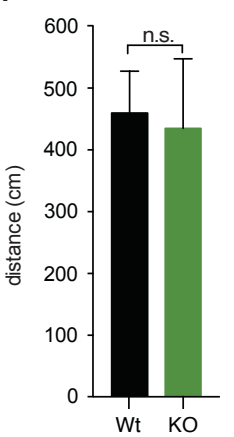

$\mathrm{D}$

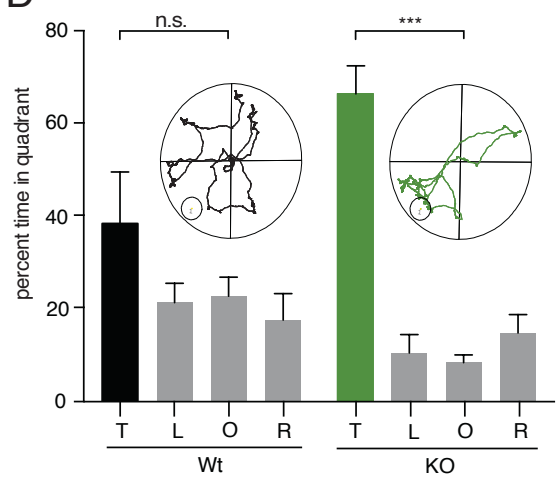

G

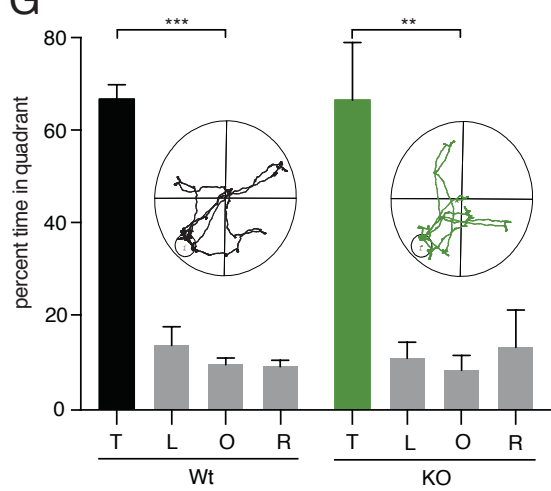

E

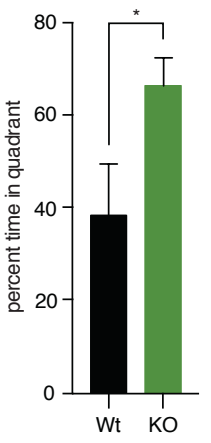

$\mathrm{H}$

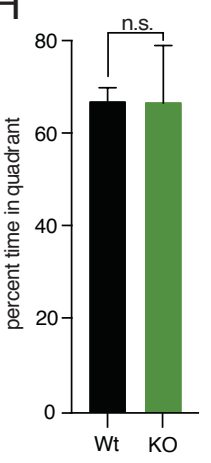

Figure 4.7: ANXA2 null mutants significantly outperformed WT in the Barnes Maze. Barnes maze (BM) testing paradigm; animals were first habituated, then trained on 3 consecutive days with one session each and a subsequent probe test (PT). Animals were allowed to rest for 2 days after PT, then trained for additional 4 days followed by a final PT (A). BM experimental setup consisted of a round white plate (92 cm diameter) and 20 holes separated into 4 quadrants. Target quadrant (T, blue) featured an escape cage hidden below target hole (green), off-target quadrants were separated into left (L), opposite $(\mathrm{O})$, and right $(\mathrm{R})(\mathrm{B})$. During the first PT both WT (black, $n=7$ ) and KO (green, $n=7$ ) travelled comparable distances $(\mathrm{cm})(\mathrm{p}=0.796$, unpaired t-test) $(\mathrm{C})$. First PT showed no significant preference of WT for T $(F(3,16)=1.76, p=0.196$, one-way ANOVA), while KO spent significantly more time in $T$ compared to $L, O, R(F(3,20)=42.68, p<0.0001$, one-way ANOVA) (D). Direct comparison of percent of time spent in target of WT and KO showed significant increase of KO over WT ( $p=0.047$, unpaired $t$-test) (E). During second PT both groups showed similar distance $(\mathrm{cm})$ travelled $(p=0.851$, unpaired $t$-test) $(F)$. Second probe test showed significant preference for $T$ in both WT $(F(3,16)=105.5, p<0.0001$, one-way ANOVA) and $\mathrm{KO}(\mathrm{F}(3,16)=12.93, \mathrm{p}=0.0002$, one-way ANOVA) as percent of time spent compared to L,O,R (G). Direct comparison of percent of time spent between WT and KO did not show significant difference during PT2 ( $p=0.990$, unpaired t-test) $(\mathbf{H})$. Error bars indicate SEM, ${ }^{*}=p<0.05,{ }^{* *}=p<0.001,{ }^{* * *}=$ $\mathrm{p}<0.0001$.

more time in the target $(F(3,20)=42.68, p<0.0001$, one-way ANOVA) (Fig. 4.7D). This difference was also significant between groups, indicating facilitated memory formation in $\mathrm{KO}(\mathrm{p}=0.047$, unpaired t-test) (Fig. 4.7E). After additional 4 days of training both cohorts were again subjected to a PT, as before displaying similar distances travelled (Fig. 4.7F). Now, also WT animals spent significantly more time in the target quadrant $(F(3,16)=105.5, p<0.0001$, one-way ANOVA), while time spent in target of $\mathrm{KO}$ did not change. $(F(3,16)=12.93, p=0.0002$, one-way ANOVA) 
(Fig. 4.7G). Also when comparing the percentage of time spent in target quadrant between groups, no difference was detected, indicating that both groups learned the task equally after 7 days of training (Fig. $4.7 \mathrm{H}$ ).

In summary, these observations are in line with previous findings and support the hypothesis that ANXA2 is involved in memory formation, acting as a repressor in hippocampus-dependent memory tasks. To understand the mechanism by which ANXA2 conveys the cognitive modulation, I investigated transcriptomic changes in hippocampal subregions of cohorts showing significantly different memory performance in the BM.

\subsubsection{ANXA2 affects the cellular membrane and RNA processing in vivo}

So far, I established a role for AnxA2 in spatial memory, potentially through hippocampus subregion-specific mechanisms. I then went on to perform a molecular characterisation of the transcriptional status of relevant brain regions in animals that I used for behavioural analysis. Before the analysis of molecular mechanisms, the animals were allowed to rest for 4 days after finishing the behavioural test battery to reinstate basal transcriptional levels. Then the animals were sacrificed and hippocampal subregions DG and CA1, both crucial for spatial memory, were isolated and used for next-generation RNA sequencing. I used both, the viral-mediated overexpression animals and the $\mathrm{KO}$ animals with their respective controls. It was surprising to see that there was no differential gene expression regardless of the AnxA2 manipulation in any of the tissues with one exception: AnxA2 in CA1 of the KO cohort at a log2FC cutoff of 0.5 (adjusted p-value (padj) < 0.05) (Fig. 4.8A). Also lower cutoffs did not lead to meaningful enrichment in pathway or overrepresentation analysis (data not shown). I was still interested in potentially smaller changes that would affect multiple genes in any given pathway, even if individual genes may not reach significance after multiple testing correction. Thus, a robust tool to study transcriptome-wide subtle changes, taking into account the full ranked gene list regardless of arbitrary cutoffs, namely gene set enrichment analysis (GSEA), was performed. This revealed several gene sets enriched in DG and CA1 of KO animals (Fig. 4.8B, Appendix Fig. 1A) but no significant difference in ANX DG or CA1 (data not shown). Importantly, GSEA in KO DG showed 5 main groups of gene ontology $(\mathrm{GO})$ categories, which I manually parsed to reduce redundancy and collapsed under the terms "membrane-bound processes", "genomic", "epigenetic", "splicing", and "RNA processing". Terms associated with each of the categories were mainly negatively enriched for $\mathrm{KO}$ with the exception of "Retrograde Vesicle Mediated Transport Golgi to ER", which showed positive enrichment. The individual GO categories highlighted two distinct mechanisms, the first targeting the cellular membrane and the latter modulating the genomic landscape and therefore possibly RNA processing. These findings indicated first hints towards potential mechanisms. The absence of differentially expressed genes could possibly be explained by non-neuronal cells or an activity-dependent mode of function. Thus, I went on to pinpoint the mechanism underlying the cognitive changes in a 
neuron specific approach and hypothesised that modification of ANXA2 would impose changes to the transcriptome when neurons were stimulated, rather than in a basal state.

A

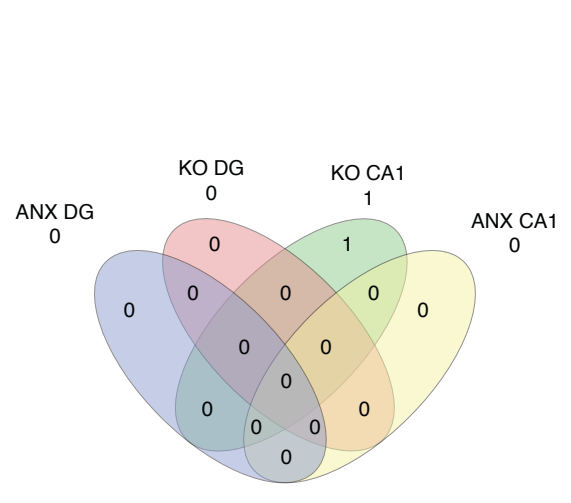

B

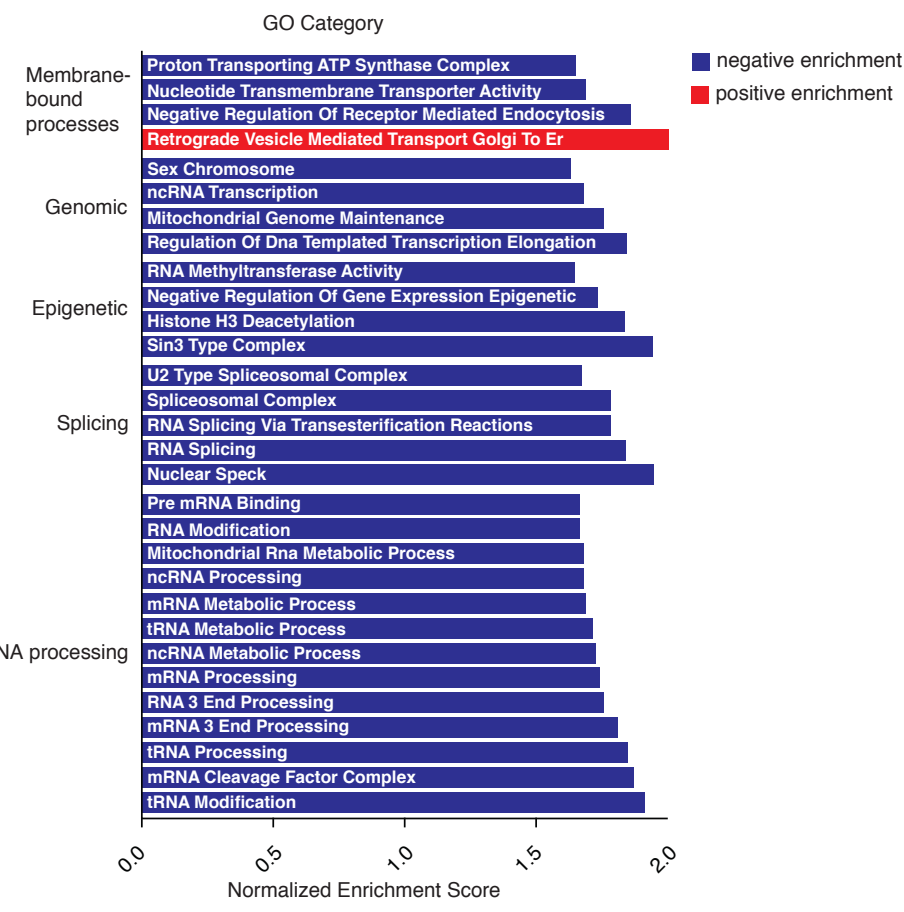

Figure 4.8: Gene set enrichment analysis reveals dual involvement of ANXA2 in membrane and RNA processing. RNAseq of ANX DG (blue) and ANX CA1 (yellow) versus GFP DG and GFP CA1 respectively and KO DG (red) and KO CA1 (green) versus DG WT and CA1 WT respectively. Analysis showed differential expression only for one gene (AnxA2) at an (Benjamini-Hochberg) adjusted pvalue $<0.05$ and log2 fold-change ( $\log 2 \mathrm{FC}) \pm 0.5$ (A). Gene set enrichment analysis (GSEA, Broad Institute) in KO DG versus WT DG revealed 30 differentially regulated gene sets (padj<0.05, FDR<0.25), manually collapsed into 'Membrane bound processes', 'Genomic', 'Epigenetic', 'Splicing', 'RNA processing'. Gene sets were sorted according to normalised enrichment score within their collapsed category. Positive enrichment (WT) is indicated in red, negative enrichment in blue (KO) (B).

\subsubsection{Neurons respond to ANXA2 overexpression by differential regulation of genes involved in developmental processes and stimulus response}

One possible reason why I only detected rather mild changes in gene expression in ANX and

NLS animals compared to GFP could be due to the fact that non-neuronal cell types could have masked changes that occur in neurons upon AnxA2 manipulation. To study the influence of ANXA2 overexpression specifically on neurons, primary neuronal cultures were infected on DIV5 with ANX-AAV or GFP-AAV as control, incubated until DIV10 and vehicle treated (Fig. 4.9A). Differential gene expression analysis in basal conditions in ANX compared to GFP overexpressing neurons showed a total of 853 differentially expressed genes at padj<0.05 (550 up, light red; 303 down, light blue) (Fig 4.9B). Over-representation analysis (ORA) of differentially regulated genes (padj $<0.05$ ) showed a strong link to neurogenesis and developmental processes (Top10 GO biological processes, padj $<0.05$, FDR $<0.05$, sorting by ratio of enrichment) (Fig. 4.9C). To investigate biologically more meaningful subsets I applied an 
additional log2 fold-change ( $\log 2 \mathrm{FC} \pm 0.5)$ cutoff, which resulted in 2 differentially expressed genes aside from $A n x A 2$. One was $\mathrm{Gda}$, which is possibly involved in microtubule assembly, the other was 6330403L08Rik a gene of unknown function. For a less conservative cutoff of padj $<0.05, \log 2 \mathrm{FC} 0.3$ only 53 genes were differentially expressed and one GO category, fibronectin binding, significantly enriched (Top10 GO molecular function, padj<0.05, FDR<0.05), pointing towards changes in the extracellular matrix (ECM) (Appendix Fig. 1B). These relatively mild differences in gene expression that affect a large number of genes could explain why transcriptome analysis found only few genes to be differentially expressed in vivo, because mild changes would have been covered by non-neuronal cells. The findings suggested that ANXA2 acts broadly but mildly on neuronal stimulus response, possibly targeting the ECM. An

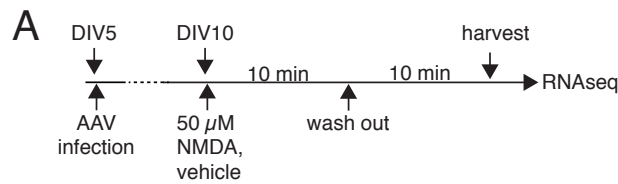

B

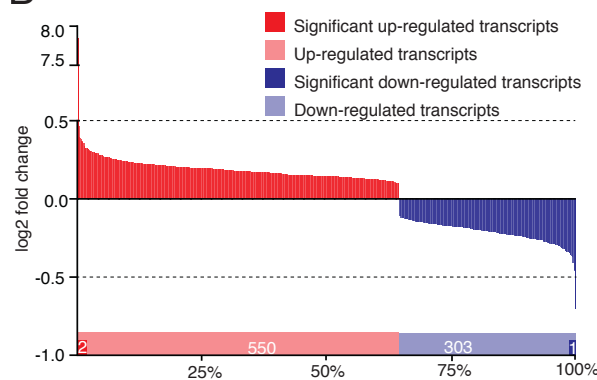

D

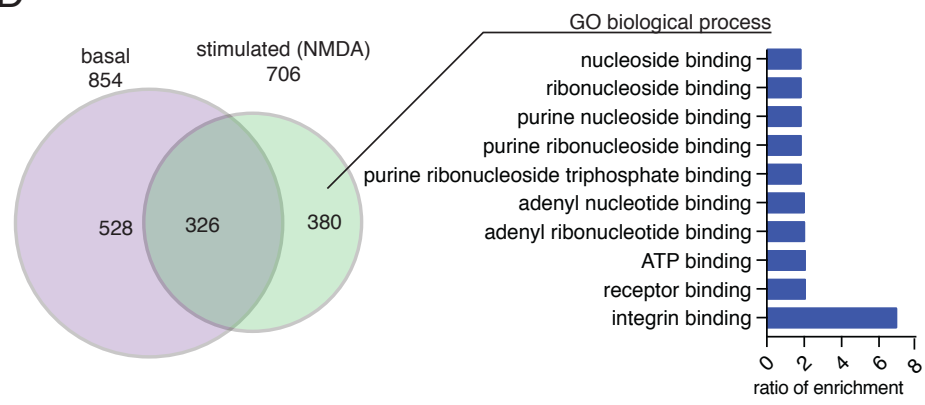

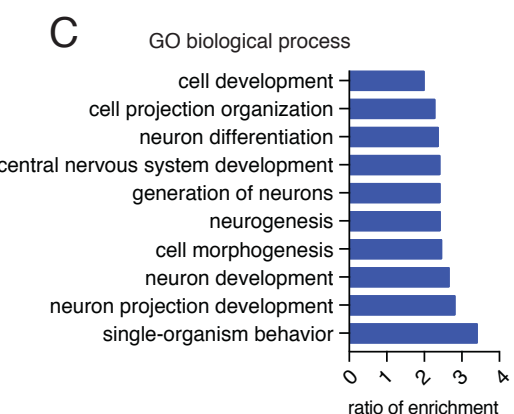

E

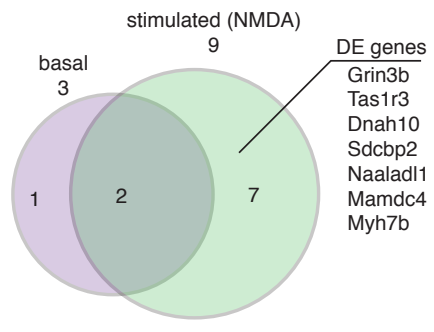

Figure 4.9: ANXA2 overexpressing neurons show wide but mild changes in gene expression and react to NMDA stimulation in an ECM-dependent manner. On DIV5 primary neurons were infected with either ANX-AAV or GFP-AAV and further incubated until simulation with vehicle (basal) or $50 \mu \mathrm{M}$ NMDA on DIV 10 for 10 min. Then medium was replaced and cultures incubated with conditioned medium to recover before harvest and RNAseq (A). Differential expression analysis after RNAseq of basal ANX versus GFP neurons showed 853 differentially expressed genes in ANX (padj<0.05; 550 up, light red; 303 down, light blue) of which 3 were significant at padj $<0.05$ and $\log 2 \mathrm{FC} \pm 0.5$ (2 up, red; 1 down, blue), $x$-axis indicates number and percent of total differentially expressed genes (B). ORA of GO biological processes (BP) for genes with at padj $<0.05$ in (B) showed neurogenesis and developmental processes and single organism behaviour (padj $<0.05$, FDR $<0.05$, sorted according to ratio of enrichment) (C). Overlap for genes differentially expressed (padj<0.05) in basal (854 genes) and NMDA stimulated (706 genes), basal signature (326 gens) was removed from stimulated condition resulting in 380 genes for ORA of GO BP. This showed nucleoside and most prominent ECM associated integrin binding GO terms enriched (padj<0.05, FDR $<0.05$, sorted according to ratio of enrichment) (D). Overlap from (D) with additional $\log 2 \mathrm{FC} \pm 0.5$ cutoff showed only 7 differentially repressed genes (Grin3b, Tas $1 \mathrm{r} 3$, Dnah10, Sdcbp2, Naalad11, Mamdc4, and Myh7b) (E). 
additional mode of action could be through mechanisms associated with RNA processing as indicated by in vivo GSEA.

Since I also hypothesised that AnxA2 might be a neuronal activity sensor and get engaged only when neurons are activated, I tested the influence of ANXA2 overexpression in challenged neurons. The ANXA2-dependent modulation of stimulus response was investigated by applying NMDA, since ANXA2 was previously reported to relocalise after NMDA treatment (Valapala, Maji et al. 2014). Cells were treated with vehicle (basal) or $50 \mu \mathrm{M}$ NMDA for 10 min followed by 10 min recovery before harvesting (Fig. 4.9A). In an effort to distinguish genes that are directly ANXA2- and activity-dependent, genes differentially expressed in basal condition were removed from GO analysis (Fig 4.9D, purple/green) and only genes exclusively deregulated in stimulated condition (padj<0.05) were analysed. ORA showed pronounced GO term enrichment for nucleoside binding processes, as well as integrin binding, pointing towards involvement of the extracellular matrix (ECM) in ANXA2-dependent stimulus response (padj<0.05, FDR<0.05, sorting by ratio of enrichment) (Fig 4.9D, green). To investigate the GO term contribution of highly differentially expressed genes, I applied an increased log2FC \pm 0.5 cutoff, which found 9 genes to be downregulated in NMDA treated neurons, of which 7 were exclusive to NMDA treatment. Among the genes detected were Grin3b (subunit of an NMDA receptor), Tas1r3 (taste receptor related to G-protein coupled receptor activity), Dnah10 (dynein, associated with ATPase activity), Sdcbp2 (involved in protein-protein interactions), Naaladl1 (related to peptidase activity), Mamdc4 (possibly involved in transport of receptors), and Myh7b (contributing nucleotide and actin filament binding) (Fig. 4.9E). Taken together these findings on ANXA2 overexpression are in line with studies on ANXA2 reporting its binding to the cytoskeleton and taking a role in trafficking (Bharadwaj, Bydoun et al. 2013). Further, analysis of all significantly deregulated genes in vitro suggested the involvement of ECM deregulation as also observed in vivo (Fig. 4.8B, Appendix Fig.1A). Based on the qualitative rather than the quantitative mode of action of chromatin readers such as ANXA2, I hypothesised that the read histone mark is already occupied to a large extend in the WT condition. Overexpression leads to an occupation of the few additional available chromatin sites. Thus, changes observed in a gain of function model must be even more pronounced in a loss of function model, given that here chromatin marks lay idle. Therefore, I investigated the effect of ANXA2 absence specifically in neurons.

\subsubsection{ANXA2 is involved in ECM and stimulus response processes}

ANXA2 deficiency lead to enhanced spatial memory in mice. To understand the effect of ANXA2 absence specifically on neurons, I investigated primary hippocampal neuronal cultures from ANXA2 null mice. Analogously to the experiments in ANXA2 overexpression, AnxA2/- $(\mathrm{KO})$ and wild-type (WT) primary neuronal cultures were incubated until DIV 10 and then treated for 10 min with either vehicle for basal analysis or $50 \mu \mathrm{M}$ NMDA for stimulation condition. The stimulation medium was then washed out and conditioned medium applied to allow the cells to 
respond before harvest and RNA sequencing (Fig. 4.10A). Differential expression analysis of KO hippocampal neurons compared to WT showed 862 genes (470 up, light red; 392 down, light blue) to be differentially regulated at padj $<0.05$. Of these genes 230 were differentially expressed at a cutoff of padj $<0.05$ and $\log 2 \mathrm{FC} \pm 0.5$ (149 up, red; 81 down, blue) (Fig. 4.10B). As expected ANXA2 deficiency led to higher fold changes for differentially expressed genes than overexpression approach in the basal state, possibly due to the lacking occupation of H4K12ac sites by ANXA2. Top $10 \mathrm{GO}$ molecular function over representation analysis with a cutoff of padj $<0.05$ and $F D R<0.05$ (sorting by ratio of enrichment) was performed, using differentially expressed genes (padj $<0.05, \log 2 \mathrm{FC} \pm 0.5$ ). This indicated the influence of ANXA2 deficiency on terms mainly associated with the ECM (blue) and cell response and activity (green). Other enriched categories, not directly associated with one of the main terms, were protein and sulphur compound binding (Fig. 4.10C). To further corroborate these findings, I investigated the transcriptome of basal KO and WT neurons using gene set enrichment analysis (GSEA). Here too, GO terms associated with the ECM were enriched in KO neurons (Top20 GO categories shown, Fig 4.10D). Moreover, terms not only associated with ECM sustainment but also disassembly were enriched. This led me to investigate more closely genes involved in both processes, and I found ECM disassembly genes to be generally increased, particularly metalloproteases (Mmp2, Mmp3, Mmp11) a family repeatedly reported to degrade collagens and to play a role in learning and memory (Terni and Ferrer 2015, Bach, Tzovara et al. 2017, Wiera, Nowak et al. 2017). Also Fn1, Nid1, and Ctsk have been linked to cognitive performance and synaptic plasticity (Dityatev and Schachner 2003, Vasudevan, Ho et al. 2010, Dauth, Sirbulescu et al. 2011) (Fig. 4.10E). Furthermore, Bgn and Lum have been reported to be important for synaptic plasticity and change due to activity and HDAC inhibition (Amenta, Creely et al. 2012, Sewal, Patzke et al. 2015). Additionally, the ECM components, and relevant modulators of synaptic plasticity Ncan and Tnc were downregulated. In contrast,

Figure 4.10: ANXA2 deficiency alters the ECM and susceptibility to stimulation.

Primary neurons from ANXA2/- or WT mice were treated for 10 min with $50 \mu \mathrm{M}$ NMDA or vehicle (basal) and allowed to recover for 10 min prior to harvest and RNA isolation for sequencing (A). RNA sequencing revealed 862 (470 up, light red; 392 down, light blue) genes differentially expressed between $\mathrm{KO}$ and WT at padj $<0.05$ cutoff. Stringent cutoff for further analysis at $\log 2 \mathrm{FC} \pm 0.5$, padj $<0.05$ showed 230 genes differentially expressed (149 up, red; 81 down, blue), $x$-axis indicates number and percent of total differentially expressed genes (B). Top10 overrepresentation analysis (ORA) for genes from (A) at $\log 2 F C \pm 0.5$, padj $<0.05$ yielded $G O$ categories associated with extracellular matrix or cell response (padj<0.05, FDR<0.05, sorted according to log10 ratio of enrichment) (C). Gene set enrichment analysis of basal neurons showed enrichment of ECM-associated GO terms within the top20 GO categories in KO when compared to WT (padj<0.05, FDR<0.25) ranking according to normalised enrichment score (NES) (D). Relative expression (normalised reads per million, NPM) showed differentially expressed genes (padj<0.05) relevant to ECM disassembly and learning and memory (e.g. Dcn, Fn1, Gsn, Ctsk, Nid1) (E). Relative expression (NPM) of ECM structure genes relevant to learning and memory and substrates of matrix-metalloproteases (MMPS) (F).Top10 ORA for molecular function GO categories in NMDA stimulated KO neurons compared to WT. Basal signature was removed (intersection) to reduce noise in gene response to stimulus. Enriched GO categories (padj<0.05, FDR $<0.05$, sorted according to log10 ratio of enrichment) in stimulated KO neurons include chromatin and DNA binding and were generally associated with cell response (green) except from macromolecular complex binding(other, black) and glycosaminoglycan binding (extracellular matrix, blue) (G). 


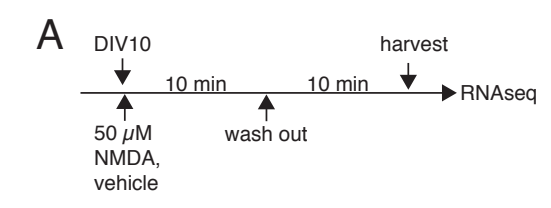

B

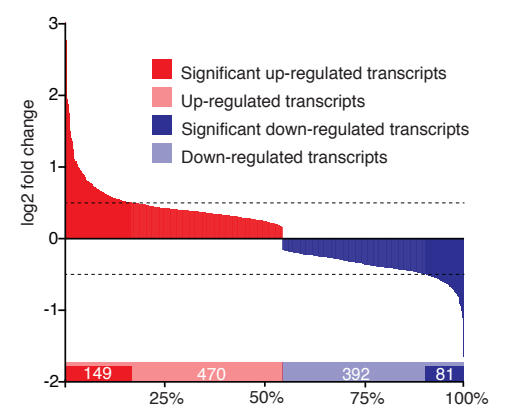

D

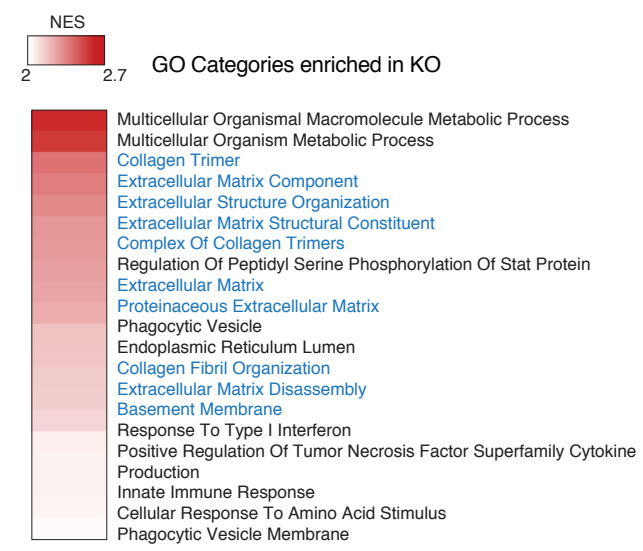

$E$

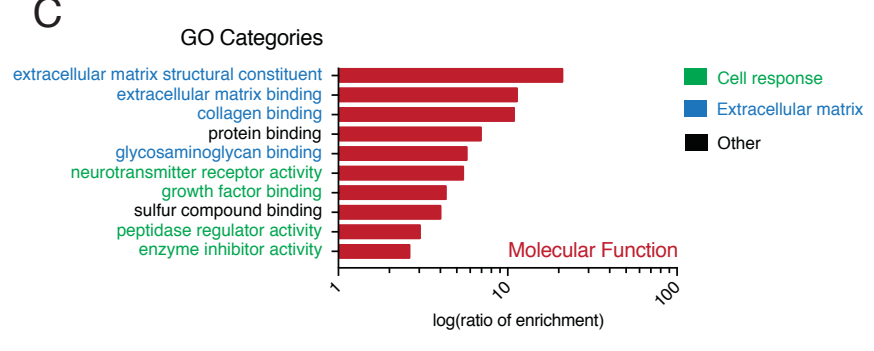

$\mathrm{F}$
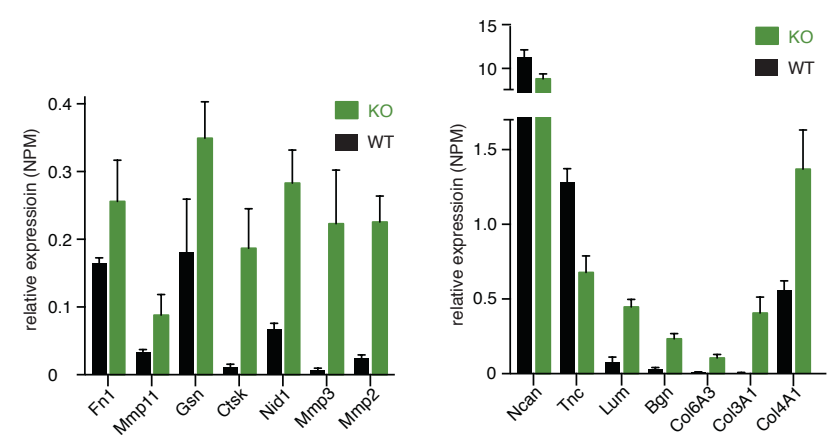

G

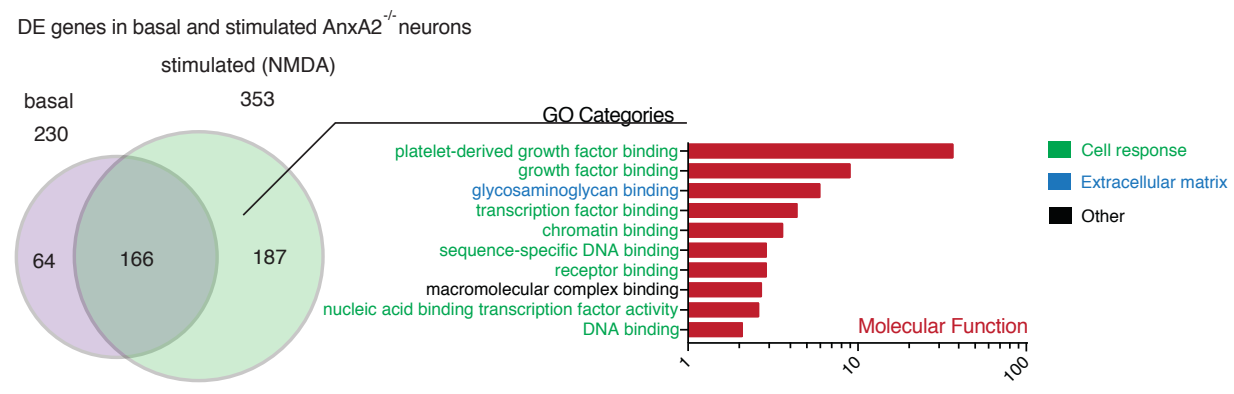

$\log 10$ (ratio of enrichment)

metalloproteases and their direct substrates such as collagen (Col6A3, Col3A1, Col4A1) were increased (Dityatev and Schachner 2003). To understand how the observed changes in ANXA2deficient neurons modulate stimulus response, I compared transcriptional changes of neurons deficient of ANXA2 to WT after NMDA stimulation. For the analysis only differentially expressed genes were used (padj $<0.05, \log 2 \mathrm{FC} \pm 0.5$ ) and the 166 genes differentially expressed in the basal state excluded (intersection) leaving 187 of total 353 significantly deregulated genes in KO compared to WT in the NMDA treated condition (Fig. 4.10G). These 187 genes matching this cutoff were then subjected to GO overrepresentation analysis to investigate molecular function (padj<0.05 and FDR $<0.05$, TOP 10 displayed sorted by ratio of enrichment). In contrast to the basal condition, only one term associated with the ECM (glycosaminoglycan binding, 
blue) and one miscellaneous (macromolecular complex binding, black) were enriched, while all other significantly enriched GO categories were closely associated with cell response including chromatin binding and DNA binding (green) (Fig. 4.10G).

In summary, the results supported the in vivo experiments, which suggested that ANXA2 had a broad but mild impact on the transcriptional response. ANXA2 was involved with gene sets associated with the ECM and could modulate the cell response upon stimulus through an alteration of chromatin and DNA binding, amongst others. AnxA2 is a multifactorial protein and reader of H4K12ac, a chromatin mark previously linked to alternative exon usage (Benito, Urbanke et al. 2015). Additionally, in vivo GSEA indicated a role in RNA processing. Thus, I investigated alternative splicing to gain a deeper mechanistic insight.

\subsubsection{NMDA but not $\mathrm{KCl}$ treatment changes exon usage in WT hippocampal neurons}

In order to get a baseline picture of splicing processes and dynamics in neuronal cells in response to a stimulus, I tested WT neurons in a stimulated condition to establish a baseline for physiological ANXA2 levels. For stimulation I chose NMDA, since ANXA2 was previously shown to be responsive to NMDA treatment (unpublished data, Kreutz M., PG Neuroplasticity, Leibniz Institute For Neurobiology, Magdeburg) (Valapala, Maji et al. 2014). Additionally, I applied KCI as an alternative stimulus to cover different mechanisms of excitation. WT neurons were treated with $20 \mathrm{mM} \mathrm{KCl}$ on DIV10 for $20 \mathrm{~min}$ and subsequently harvested and subjected to RNA sequencing to analyse differentially used exons bits (herein exons), using DEXseq (Fig. 4.11A). Neurons treated with $20 \mathrm{mM} \mathrm{KCl}$ showed three significantly included exons (Pcdh8, GO category "calcium ion binding", Dusp1 GO category "phosphatase activity"; Rpl41, GO category "RNA binding"; padj<0.05, $\log 2 \mathrm{FC} \pm 0.2$ ). Exon exclusion was not detected compared to basal condition (Fig. 4.11B). In contrast, stimulation with $50 \mu \mathrm{M}$ NMDA for 10 min with 10 min recovery in conditioned medium prior to harvest (Fig. 4.11C) invoked a total of 719 differentially used exons (310 included, light red; 409 excluded, light blue) with padj<0.05. At an additional cutoff of $\log 2 \mathrm{FC} \pm 0.2148$ exons (68 included, red; 80 excluded, blue) were differentially expressed. This subset was used for further analysis (Fig. 4.11D). Pathway analysis of differentially used exons (padj $<0.05, \log 2 \mathrm{FC} \pm 0.2$ ) showed significant clusters (padj<0.05) associated with neuronal activity such as neurotransmitter secretion, action potential and elongation. Intriguingly, histone methylation (specifically including $\mathrm{H} 3 \mathrm{~K} 4 \mathrm{me}$, associated with active transcription) was the main cluster enriched for differential exon usage. Taken together, this data showed a specific response in alternative exon usage upon NMDA but not $\mathrm{KCl}$ treatment in neurons, targeting pathways associated with epigenetics and learning and memory. Since, this demonstrates the difference of alternative exon usage in response to stimulus, I went on to study the effect of ANXA2 on exon usage during NMDA stimulation. 
A

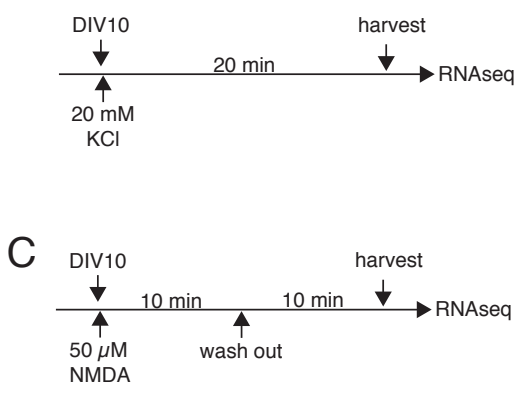

B

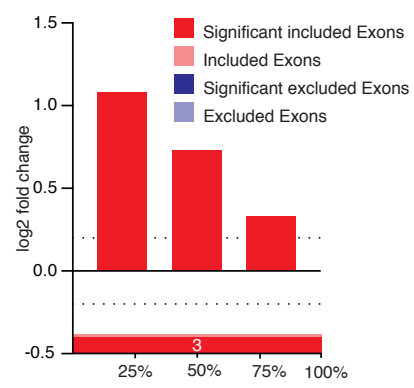

$\mathrm{D}$

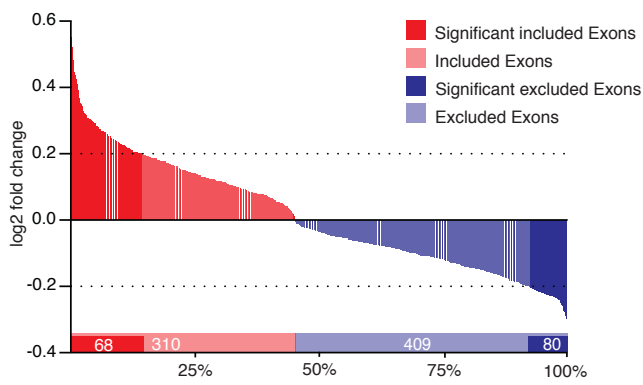

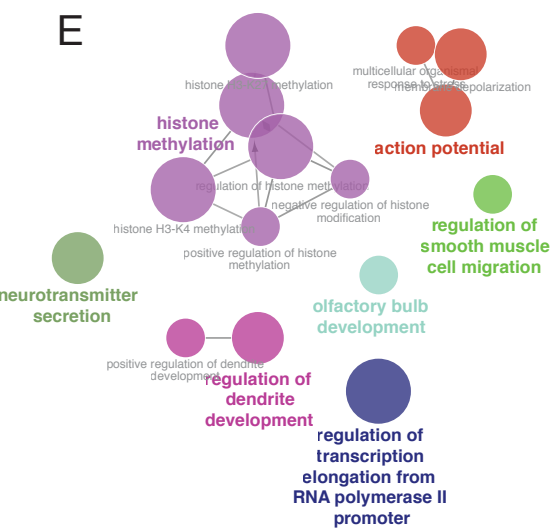

Figure 4.11: NMDA but not $\mathrm{KCl}$ treatment induces alternative exon usage in primary neuronal culture. $20 \mathrm{mM} \mathrm{KCl}$ treatment was applied to DIV10 neurons for $20 \mathrm{~min}$. Subsequently, neurons were harvested and subjected to RNA sequencing $(\mathbf{A})$. Neurons treated with $\mathrm{KCl}$ included exons for only 3 genes (Pcdh8, Dusp1, and Rpl41) in a significantly different manner (padj<0.05, log2FC \pm 0.2 , genes in descending order of log2FC). (B). $50 \mu \mathrm{M}$ NMDA treatment was applied to neurons at DIV10 for $10 \mathrm{~min}$. Neurons were allowed to recover for $10 \mathrm{~min}$ in conditioned medium prior to harvesting and RNA sequencing (C). DEXseq showed 719 exons differentially used between basal and stimulated neurons (310 included, light red; 409 excluded, light blue; padj<0.05). At an additional log2FC \pm 0.2 cutoff 148 exons were differentially expressed (68 included, red; 80 excluded, blue; padj $<0.05$, log $2 \mathrm{FC} \pm 0.2$ ), $x$-axis indicates number of differentially used exons and percentage of total exons differentially expressed (D). ClueGO pathway analysis of 148 differentially expressed exons of (D) (padj<0.05, log2FC \pm 0.2 ) showed stimulus response and epigenetic pathways were enriched (padj<0.05), proximity within cluster represents interaction strength, circle size represents padj (E).

\subsubsection{ANXA2 overexpression and deficiency show opposing effects on exon usage and target pathways crucial for neuronal signal transduction}

ANXA2 is a reader of $\mathrm{H} 4 \mathrm{~K} 12 \mathrm{ac}$, a chromatin mark previously linked to differential splicing and cognitive decline in ageing (Benito, Urbanke et al. 2015). Naturally, I asked how this change in splicing is conveyed from a histone PTM to translation. Since, in vivo GSEA implicated involvement of ANXA2 in splicing and RNA processing, ANXA2 could read the histone modification and initiate the respective transcriptional machinery leading to alterations in exon usage, ultimately contributing to the behavioural phenotype observed.

To explore this, I used the same ANXA2 overexpression samples previously investigated for differential expression (Fig. 4.9A). Differential exon usage analysis showed 207 exons to be differentially expressed at padj<0.05. When an additional log $2 \mathrm{FC} \pm 0.2$ cutoff was applied, 150 exons were regulated significantly different. Interestingly, the majority of these exons (123, blue) 
were skipped, while only few (27, red) were included compared to GFP control (Fig. 4.12A). In comparison to differential gene expression (3 differentially expressed genes), differential exon usage was more pronounced (122 genes with 150 differentially expressed exons). In addition, differential expression was mutually exclusive with the exception of one gene (AnxA2, E021 due to fusion to GFP), when comparing genomic identifiers (Fig. 4.12B). ClueGO analysis of differentially expressed exons in basal ANXA2 overexpressing neurons showed terms associated with microtubule fibre structure (regulation of supramolecular fibre organisation, negative regulation of supramolecular fibre organisation, regulation of microtubule (de-)polymerisation) and neuron projection extension (padj<0.05). This pointed towards an alteration in neuronal cytoskeletal structure, crucial for appropriate signal transmission

A

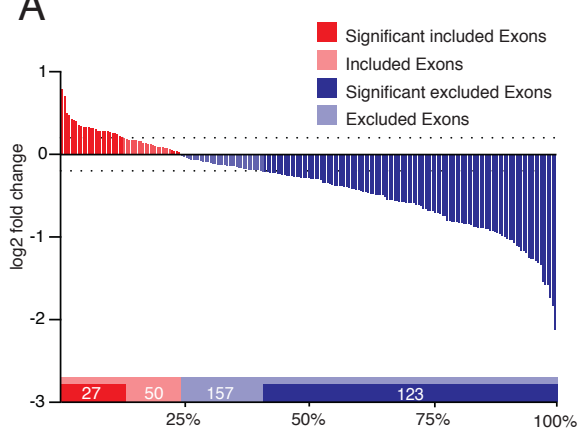

B

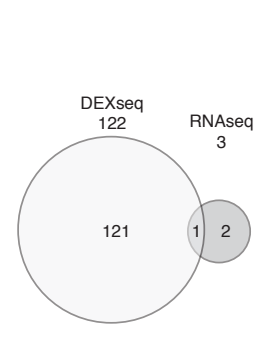

C

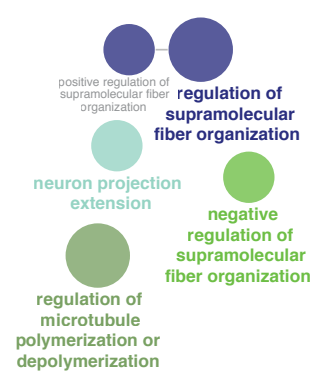

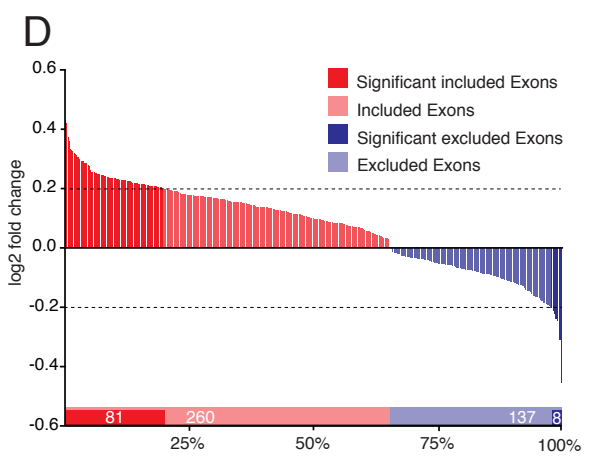

$E$

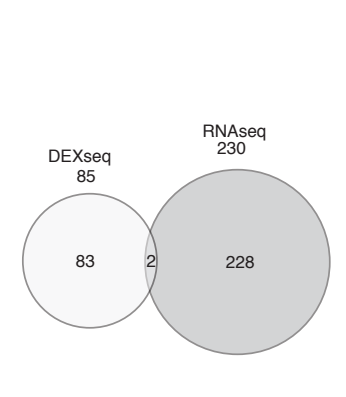

$\mathrm{F}$

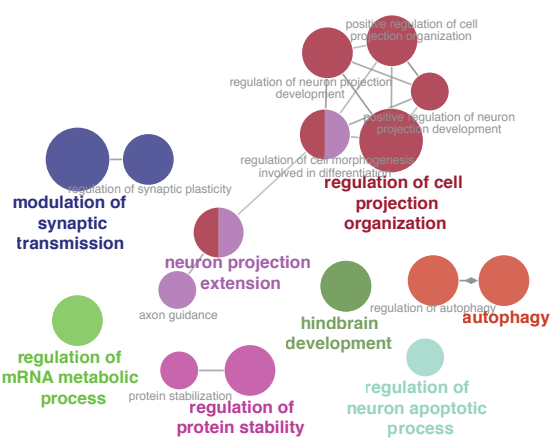

Figure 4.12: Differential exon usage between ANXA2 overexpression and deficiency is inverted and targets structural genes associated with neuronal projection.

Differential exon usage analysis in AnxA2 overexpressing neurons compared to GFP showed a total of 207 exons differentially expressed. The majority of exons, $>75 \%$, were skipped (50 included, light red; 157 skipped, light blue; padj<0.05). Of these, 150 (27 included, red; 123 skipped, blue; padj<0.05, $\log 2 \mathrm{FC} \pm 0.2$ ) exons were differentially expressed at an additional $\log 2 \mathrm{FC} \pm 0.2$ cutoff $(\mathbf{A})$. Comparison of genomic identifiers (GIs) from differential exon usage (padj<0.05, log2FC \pm 0.2 ; DEXseq, 122 GIs) and differential gene expression (padj $<0.05, \log 2 \mathrm{FC} \pm 0.5$; DEseq, $3 \mathrm{Gls}$ ) showed overlap for one gene (AnxA2) (B). ClueGO biological process (BP) analysis of 150 differentially expressed exons of (A) showed GO terms associated with microtubule fibre structure and neuron projection extension (padj<0.05) (C). Differential exon usage analysis in Anxa2 KO neurons compared to WT showed $>70 \%$ exon inclusion with a total of 397 exons differentially expressed (260 included, light red; 137 skipped, light blue; padj<0.05). Of these, 88 exons were differentially expressed at an additional $\log 2 \mathrm{FC} \pm 0.2$ cutoff (81 included, red; 8 skipped, blue; padj<0.05, log2FC \pm 0.2 ) (D). Comparison of Gls from differential exon usage (padj $<0.05, \log 2 \mathrm{FC} \pm 0.2$; DEXseq, $83 \mathrm{Gls}$ ) and differential gene expression (padj<0.05, log2FC \pm 0.5 ; DEseq, 230 Gls) showed overlap for two genes (Tmod3, Gfap) (E). ClueGO analysis of 88 differentially expressed exons in (D) showed GO BP terms enriched associated with neuron development, neuron projection and synaptic transmission (padj<0.05) (F). 
(Fig 4.12C). In a complementary approach, I investigated the influence of ANXA2 deficiency on splicing. To this end, I analysed the RNAseq results from previous differential gene expression analysis of KO neurons with and without NMDA stimulus for differential exon usage. This revealed differential regulation of 397 exons at padj<0.05 of which 89 were considered significant at an additional $\log 2 \mathrm{FC} \pm 0.2$ cutoff. Importantly, in direct contrast to overexpression experiments ANXA2 deficiency lead to major exon inclusion (81, red) and few skipped exons (8, blue) essentially mirroring the previous experiment (Fig. 4.12D). Here too differential exon usage and differential gene expression showed few commonly deregulated genes (Tmod3, E001; Gfap, E006) when comparing genomic identifiers (Fig. 4.12E). Pathway analysis of differentially used exons (padj $<0.05, \log 2 \mathrm{FC} \pm 0.2$ ) using ClueGO showed mainly enriched $G O$ terms associated with metabolism, autophagy, synaptic transmission, and neuronal projection (Fig. 4.12F). Notably, differential exon usage in overexpression and knockout experiments showed inverted ratios between exon skipping and inclusion. However, altered genes contribute to closely related GO categories associated with neuronal structures necessary for signal transduction.

\subsubsection{ANXA2 manipulation changes NMDA-dependent splicing response}

Finding significantly altered exon usage in both ANXA2 overexpression and deficiency in the basal state, was highly interesting. Particularly the finding that associated GO terms strongly suggest changes in structural composition was intriguing, since this is a necessary basis for neuronal signal transduction. Thus, I investigated whether this structural change would influence the cellular response to challenged conditions. To this end, I stimulated primary neuronal hippocampal cultures overexpressing or deficient of ANXA2 with NMDA or vehicle and subjected them to RNA sequencing, as previously described (Fig 4.9A).

As shown beforehand, overexpression and ANXA2 deficiency led to significantly changed exon usage, thus, I removed the basal state for both stimulated conditions. Doing so I also showed that differentially regulated exons in the basal states were mostly distinct when comparing overexpression and $\mathrm{KO}$. The only overlapping genes were Nflx (GO annotation: transcription factor activity, sequence-specific DNA binding), Supt5h (GO annotation: poly(A) RNA binding and chromatin binding) and Map6 (GO annotation: microtubule binding) (Fig. 4.13A). Notably, Rusc2 was the only gene showing differential exon usage in all conditions and was closely linked to intellectual disability and microcephaly (Abramowicz 2016, Alwadei, Benini et al. 2016). When comparing stimulated conditions of $A n x A 2 \mathrm{KO}$ and overexpression common genes showing differential exon usage were Ankrd13b (may regulate EGFR internalisation), HNRNPA2B1(GO annotations: nucleic acid binding and RNA binding; acts as a nuclear reader of the N6-methyladenosine), Map4 (GO annotations: poly(A) RNA binding and microtubule binding), and Mapt (GO annotations: protein kinase binding and microtubule binding) (Fig. 4.13A). 


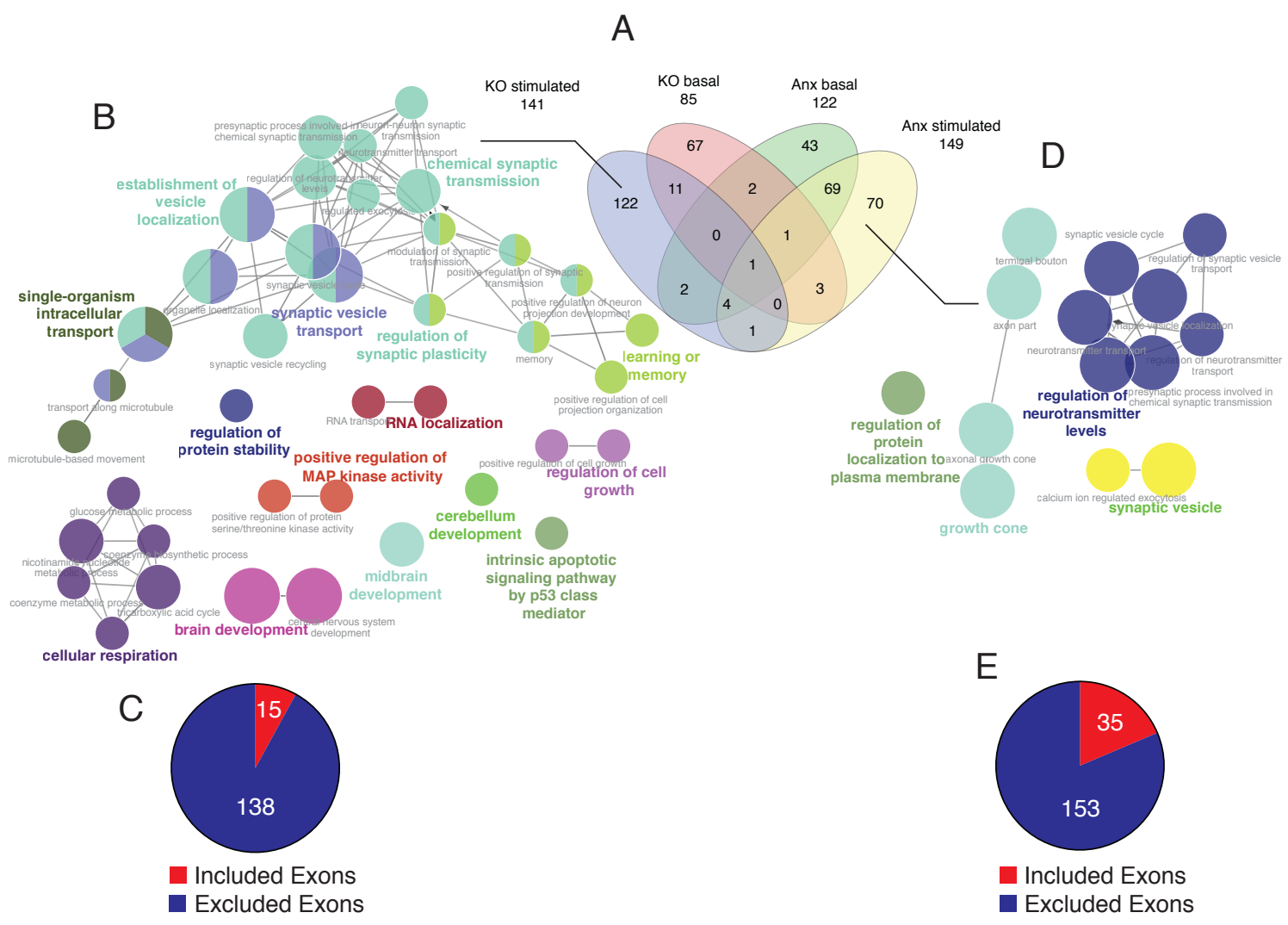

Figure 4.13: NMDA stimulation of AnxA2 overexpressing or deficient neurons changes exon usage in genes relevant to synaptic plasticity.

Venn diagram of differentially used exons of all 4 tested conditions showed little overlap for ANXA2 overexpression and KO. Stimulation with NMDA introduced alternative exon usage in both KO and overexpression compared to basal state (A). Stimulation with NMDA in AnxA2 KO neurons induced alternative exon usage mainly in genes associated with synaptic vesicle transport and learning and memory after removal of basal differentially expressed genes (B). Of differentially expressed exons in NMDA stimulated KO neurons 138 exons were excluded while 15 were included (C). NMDA stimulation of neurons overexpressing ANXA2 showed differential exon usage in genes mainly associated with regulation of neurotransmitter release and growth cone (axon guidance) (D). Of differentially expressed exons in NMDA stimulated ANXA2 overexpressing neurons 153 exons were excluded while 35 were included (E). All exons used in the analysis were significant at padj $<0.05, \log 2 \mathrm{FC} \pm 0.2$.

As expected, the majority of differentially regulated exons was found after NMDA stimulation in both ANXA2 overexpression and KO. After removal of exons differentially expressed in the basal state AnxA2 KO neurons showed differential exon usage in genes associated with development, cellular regulation, RNA localisation, and cellular respiration. Further, the main cluster formed around synaptic vesicle transport including intracellular transport, chemical synaptic transmission, regulation of synaptic plasticity and learning or memory (ClueGO, padj<0.05, used gene set indicated) (Fig. 4.13B). Notably, of differentially expressed exons 138 were excluded while 15 were included in the NMDA stimulated condition (Fig. 4.13C). Accordingly, I analysed differential exon usage in neurons overexpressing ANXA2 and stimulated with NMDA as described beforehand. After removal of the basal signature, GO term analysis showed significant enrichment (padj<0.05) in categories associated with protein localisation, growth cone, synaptic vesicle, and regulation of neurotransmitter levels (Fig. 4.13D). In this analysis, the majority of 153 differentially used exons was excluded and 35 
included (Fig. 4.13E). These findings strongly suggested that ANXA2 is closely involved in alternative exon usage specifically in genes relevant for synaptic plasticity and in turn learning and memory. Intriguingly, the terms enriched in both $\mathrm{KO}$ and overexpression are complementary, yet more pronounced in $\mathrm{KO}$, also represented by numbers of genes expressed. Notably, this finding was also reflected in the initially observed phenotype in AnxA2 KO and overexpression. The obvious question was how the alternative exon usage similar in overexpression and KO would induce opposing behavioural phenotypes. To elucidate this question I analysed the properties of alternatively used exons.

\subsubsection{ANXA2 regulates energy metabolism by alternative 5' usage in a H4K12ac- associated manner}

ANXA2 overexpression and deficiency showed inverted ratios in alternative exon usage. However, NMDA stimulation lead to significant exon skipping, affecting related biological processes in both mutations. To understand how a seemingly similar mechanism contributes to opposing behavioural phenotypes I plotted each changed exon as percentage of the total maximum transcript length at the relative location in the gene body which was divided into 100 bins starting from 0 . Additionally, I plotted H4K12ac ChIP peaks, which showed enrichment around the TSS. I hypothesised that the peaks must correlate to exons alternatively used in ANXA2 mutant, if the exon usage changes are causally linked to ANXA2 in a H4K12acdependent complex.

Plotting NMDA treated WT compared to control showed alternative exon usage along the entire gene body with little enrichment towards the 3 ' end of gene bodies (100) and did not coincide with H4K12ac signature (Fig 4.14A). NMDA stimulated ANXA2 overexpressing neurons compared to basal state showed increased alternative exon usage along the gene body and 3', also a subtle increase of alternatively used exons was observed towards the TSS (Fig 4.14B). Importantly, when comparing NMDA-treated AnxA2 KO to unstimulated control, I observed few differentially used exons along the gene body, while I found substantial deregulation coinciding with H4K12ac peaks (Fig 4.14C). Merging the alternative exon usage signatures of each condition underlined the enrichment along the gene body towards the 3' UTR in WT and overexpression but not KO, which showed enrichment around H4K12ac peaks (Fig. 4.14D). Importantly, 51\% of alternative exon usage in ANXA2 deficient neurons was restricted to the first $10 \%$ of the gene body, while the rest of the gene bodies harboured $49 \%$ of total differentially used exons and showed no enrichment for any KEGG pathways. Analysis of genes associated with the H4K12ac peak in NMDA stimulated KO revealed KEGG pathways related to energy metabolism such as citrate cycle, pyruvate metabolism, glycolysis but also diseases such as Parkinson's disease and AD (Fig. 4.14E).

Taken together these findings suggest that ANXA2 acts as a reader of H4K12ac, not only contributing to differential exon usage in genes associated with learning and memory but also 
by specifically targeting alternative $5^{\prime}$ exon usage in genes closely associated with energy metabolism. How precisely this contributes to improved spatial memory function remains to be elucidated. However, one can speculate that the alternative 5' usage in AnxA2 KO neurons could impact the regulation of protein synthesis through altered ribosomal recruitment (Filbin and Kieft 2009, Rojas-Duran and Gilbert 2012), thus generating an energetically favourable environment.

A

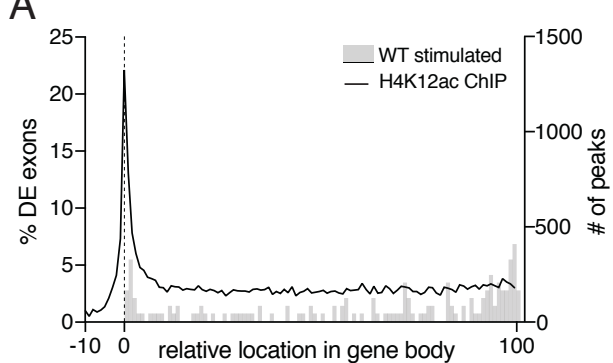

$\mathrm{C}$

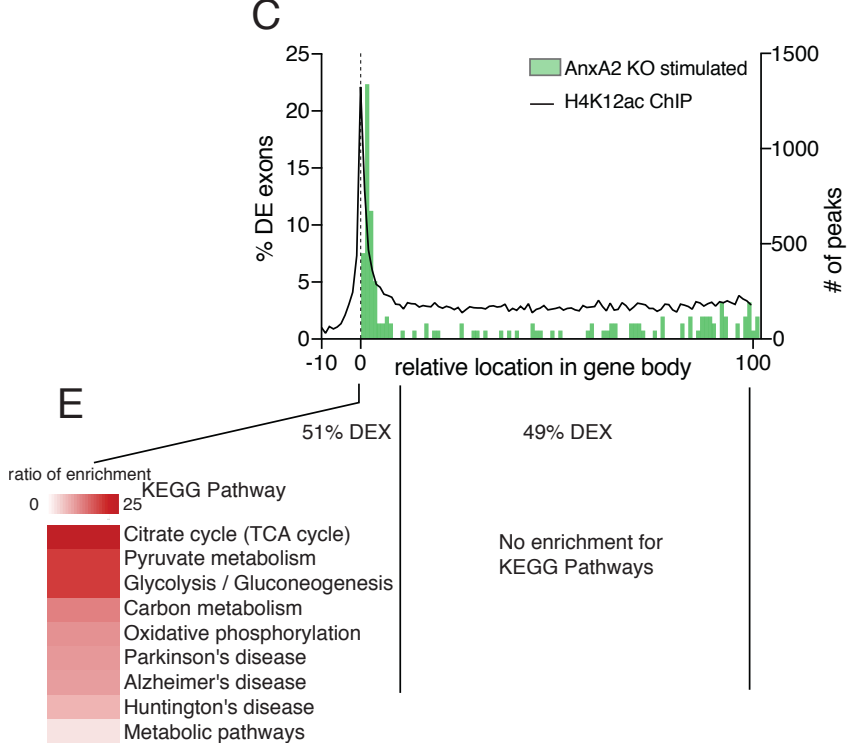

B

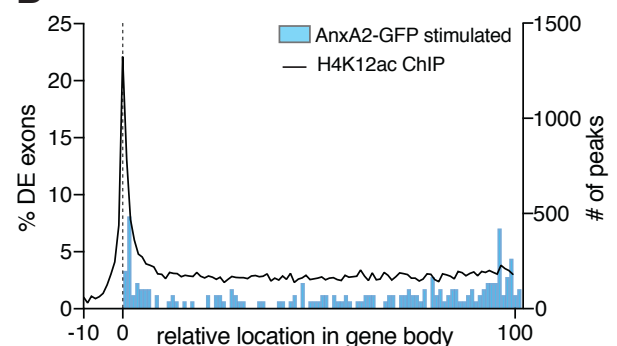

D

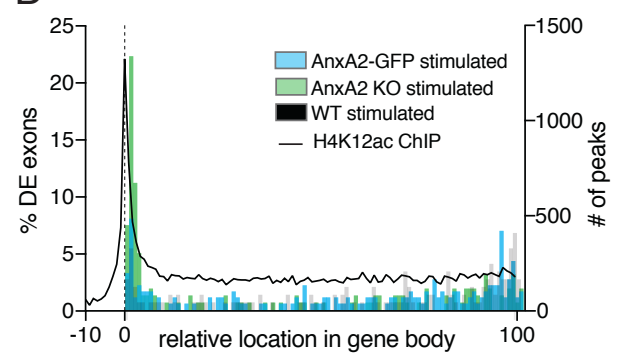

Figure 4.14: ANXA2 regulates energy metabolism by alternative 5 ' usage in an H4K12ac associated manner.

Histogram of percent of differentially used exons (left y-axis) in treated neurons compared to basal state, and number of H4K12ac ChIP peaks (right y-axis) both illustrated relative to gene body length $(-10 \%$ $0 \%-100 \%)$ for NMDA treated WT neurons (A), NMDA treated ANXA2 overexpressing neurons (B), NMDA treated KO neurons (C), and merge of A-C (D). KEGG pathway analysis ( $<<0.05, F D R<0.05)$ for exons alternatively used $10 \%$ downstream of TSS showed energy metabolism pathways. Same analysis for exons differentially expressed in $10 \%-90 \%$ gene body length showed no enrichment for KEGG pathways $(p<0.05$, FDR $<0.05)(E)$. All exons used in the analysis were significant at padj $<0.05$, $\log 2 \mathrm{FC} \pm 0.2$. 


\subsection{Novel treatment strategies for Alzheimer's Disease (AD)}

I targeted the two essential molecular pathologies of Alzheimer's disease in two lines of experiments by testing novel agents to ameliorate the pathogenesis. Patients suffering from $A D$ display $A \beta$ plaques and neurofibrillary tangles (NTFs) but also significantly increased HDAC6 levels are observed. Reducing HDAC6 was previously reported to reinstate memory impairments in a mouse model for AD-like amyloidosis (Govindarajan, Rao et al. 2013). Further, HDAC6 reduces microtubule acetylation levels, decreasing their stability and deacetylates Tau, promoting its phosphorylation and aggregation. Thus, the first set of experiments targeted HDAC6 inhibition and deficiency to increase TAU and microtubule acetylation, thereby ameliorating the pathology induced by NTFs. In the second set of experiments we tested the novel compound anle138b. The compound was previously reported to be beneficial in different aggregopathies, including a model for AD-like tauopathy (Wagner, Krauss et al. 2015). In our experiments we used anle138b to targeted amyloid beta $(A \beta)$ induced pathology by pharmacologically inhibiting membrane pores caused by $A \beta$ oligomers.

\subsubsection{Targeting the interaction between TAU and HDAC 6 to ameliorate $A D$-like tauopathy}

In several instances the decrease of TAU acetylation has been demonstrated to promote TAU aggregation (Cook, Gendron et al. 2012, Noack, Leyk et al. 2014, Selenica, Benner et al. 2014). Microtubuli and TAU were shown to be a direct substrate of HDAC6 (Hubbert, Guardiola et al. 2002, Ding, Dolan et al. 2008). This suggests that inhibiting HADAC6 would increase TAU and microtubuli acetylation and in turn reduce TAU deposition while enhancing microtubuli stability. Additionally, increasing acetylation levels through HDAC6 removal has proven beneficial in mitigating the pathology in models for $A \beta$ amyloidosis, one of the two pathological molecular
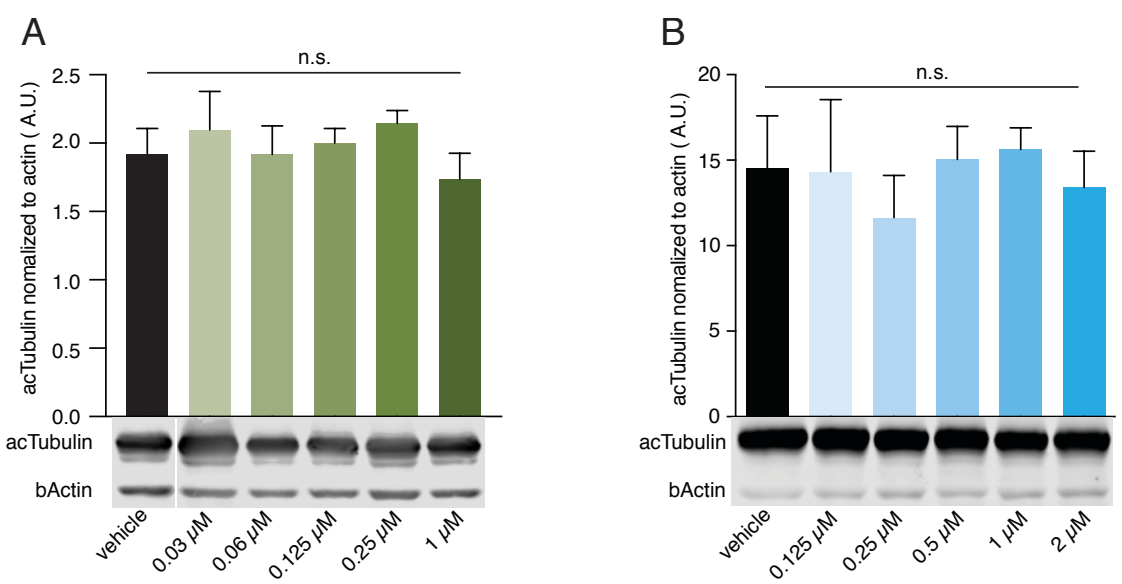

Figure 4.15: JS28 does not change tubular acetylation in PNC.

Western blot analysis of primary hippocampal neurons treated for $24 \mathrm{~h}$ with JS28 at concentrations from $0.03 \mu \mathrm{M}$ to $1 \mu \mathrm{M}$ did show changes in tubular acetylation normalised to $\beta$-actin (A). Additional Western blot from primary hippocampal cultures after $24 \mathrm{~h}$ JS28 application at concentrations from $0.125 \mu \mathrm{M}$ $2 \mu \mathrm{M}$ normalised to $\beta$-actin (B). No change could be observed. Error bars indicate SEM. 
hallmarks of AD (Govindarajan, Rao et al. 2013). To investigate whether HDAC6 could be a feasible drug target in the other molecular hallmark of $A D$, namely TAU deposition and neurofibrillary tangles (NFT), I tested the effect of HDAC6-dependent acetylation in a model for AD-like tauopathy. To this end, I tested the novel compound JS28, which was suggested to specifically inhibit HDAC6 and in turn increase tubular acetylation levels at nano molar concentration (unpublished data, Senger and Jung). In contrast to this report from cell lines, I found JS28 not to alter tubular acetylation in primary hippocampal culture as demonstrated in Western blot. Therefore JS28 was not a suitable compound for further in vivo testings (Fig. 4.14A+B).

Since these results ruled JS28 testing in vivo, I took an alternative approach and generated a genetic in vivo model. Therefore, I crossed heterozygous male mice of the THY-Tau22 mouse model for AD-related Tau pathology expressing the humanised quadruple repeat tau, with heterozygous Hdac6 knockout females. The resulting male wild-type (control); Tau ${ }^{+/ T}$, Hdac6-y (HDAC6 KO); Tau+r , Hdac6-/y (TAU/HDAC6 KO) or Tau+/T, Hdac6 ${ }^{+/ y}$ (Tau) offspring was tested. Since Tau pathology progresses age-dependently, control, HDAC6 KO, Tau/HDAC6 KO, and Tau mice were aged for 6 or 13 months to generate two cohorts, an early-stage and a late-stage of the phenotype (Fig 4.15A). Basic anxiety, motor and cognitive performance were assessed in a behavioural test battery starting with anxiety tests (open field, elevated plus maze), followed by a spatial navigation and memory task (Morris water maze) and classical pavlovian fear memory test (contextual fear conditioning) (Fig 4.15B).

\subsubsection{Anxiety is modulated by Hdac6 deficiency in THY-Tau22 transgenic mice.}

At 6 months of age mice expressing the TAU transgene spent significantly more time in exposed areas $(F(1,52)=10.92, p=0.002$, 2-way ANOVA), indicating less anxious behaviour in the open field. Post-hoc testing found the increase of anxious behaviour to be significant when compared to HDAC6 KO ( $p=0.037$, Tukey). No interaction between the transgenes was observed

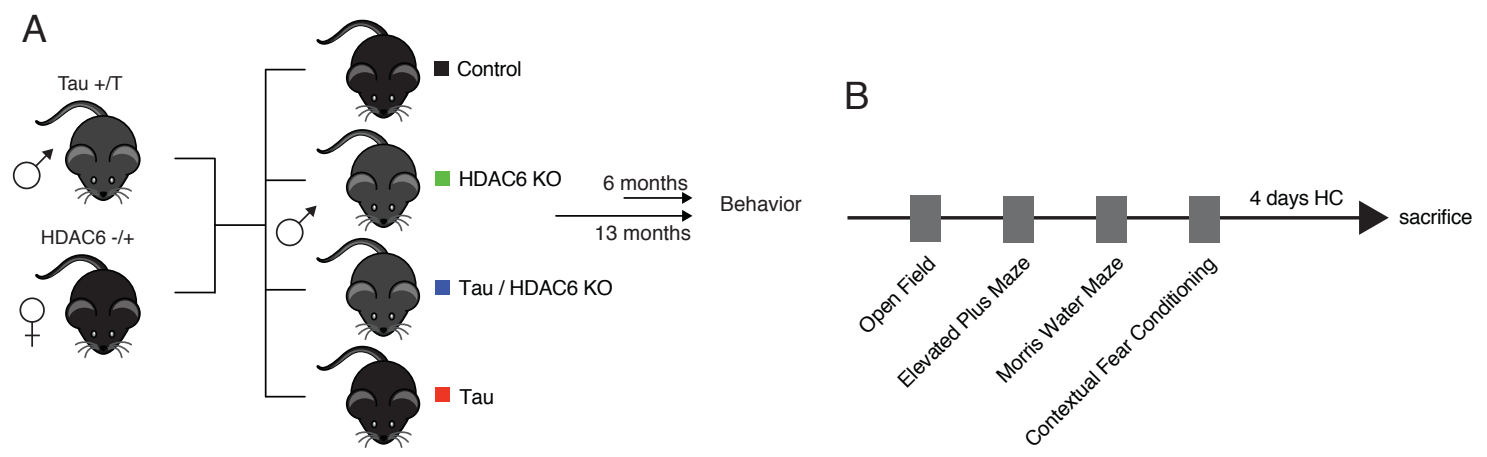

Figure 4.16: HDAC6 and TAU mating scheme and behavioural paradigm.

Mating scheme of parental transgenic mouse lines and resulting offspring genotypes, Tau ${ }^{+/+}, \mathrm{Hdac}^{+/ y}$ (control, $\mathrm{n}=15$ ); TAU ${ }^{1 /+}, \mathrm{HDAC6}^{-/ y}$ (HDAC6 KO, n=15); TAU+/T, Hdac6-/y (TAU/HDAC6 KO, n=20) or TAU+1 T, $H D A C 6^{+/ y}$ (Tau, $\mathrm{n}=15$ ). Cohorts were tested after 6 and 13 months respectively (A). The behavioural test battery was performed for open field test, elevated plus maze, Morris water maze and contextual fear conditioning on consecutive days. Afterwards, animals were returned to home-cage ( $\mathrm{HC})$ for 4 days before sacrifice (B). 
(Fig. 4.16A). To investigate potential motility differences and to further rule out motor impairments common in most Tau pathology models, travelling speed in the open field was measured. This did not show a significant difference between Tau and control and confirmed that the TAU transgene in this model does not contribute to motor deficits in the open field test, which is in line with previous reports (Schindowski, Bretteville et al. 2006, Van der Jeugd, Blum et al. 2013) (Fig. 4.16A). I found travel speed was increased dependent on the expression of the Tau transgene $(F(1,61)=7.14, p=0.01$, 2-way ANOVA). Again, this observation was found to be significantly different in post-hoc testing when HDAC6 KO were compared to Tau/HDAC6 KO littermates ( $p=0.025$, Tukey) (Fig. 4.16B). To assess the observed anxiety phenotype in more detail, I performed an elevated plus maze test. In contrast to a previous report (Schindowski, Bretteville et al. 2006), no anxiety phenotype could be observed at an early-stage time-point in Tau. All groups spent significantly more time in the closed arms and no difference between groups could be detected (Fig. 4.16C).

\subsubsection{Absence of HDAC6 modulates hippocampus-dependent memory in the early- stage AD-like tauopathy model.}

Aside from increased anxious behaviour, the expression on quadruple repeat Tau was reported to cause impairment in spatial memory (Van der Jeugd, Blum et al. 2013). To test if HDAC6 deficiency has a beneficial effect on cognitive function in the Tau pathology model, I conducted the Morris water maze test for spatial navigation and memory, a highly hippocampus-dependent task. Animals were trained on consecutive days during which the latency to find the submersed platform (escape latency) was taken as a measure for learning performance. Probe tests (PT) were performed when one of the cohorts reached a plateau in their performance. As expected, control animals showed the lowest escape latency, indicative of fast memory acquisition. However, I did not observe significant differences between the groups for the early-stage cohorts $(F(3,40)=1.034, p=0.388$, 2-way ANOVA), which is in line with my previous findings in the elevated plus maze test where I did not detect the previously reported anxiety phenotype (Schindowski, Bretteville et al. 2006) (Fig. 4.16D).

To elucidate the role of HDAC6 deficiency in cognition, I performed an additional memory test, namely contextual fear conditioning, a hippocampus-dependent classical pavlovian conditioning paradigm with additional involvement of the amygdala. As expected, during training all groups displayed comparable initial freezing. When the animals were tested after the conditioning stimulus during recall, a significant difference between the groups based on HDAC6 deficiency was detected using 2-way ANOVA $F(1,63)=7.84, p=0.007)$. Importantly, no interaction between the transgenes was observed. In turn, controls and Tau did not show differences in freezing behaviour (Fig. 4.16E). Taken together these findings indicate that the Tau22 mouse model shows increased activity but no anxiety phenotype compared to wild-type controls at 6 months of age. The model does not show spatial or contextual fear memory impairments at 6 months of 
age. Notably, HDAC6 deficiency impacts contextual fear memory in Wt and TAU transgenics, suggesting a beneficial effect in the presence of a pathological cognitive deficit.

A

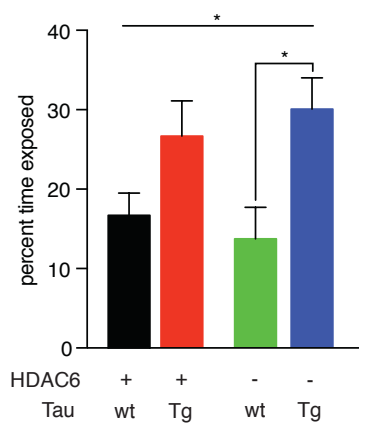

B

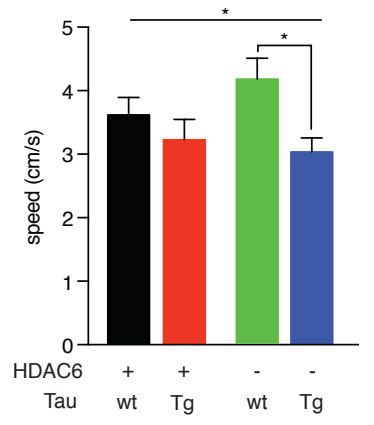

C

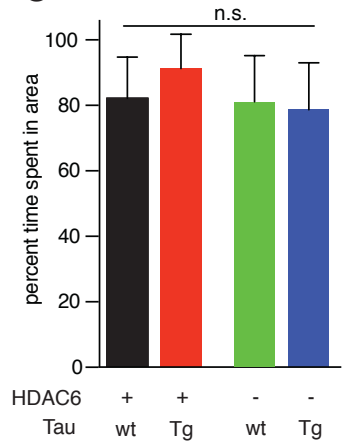

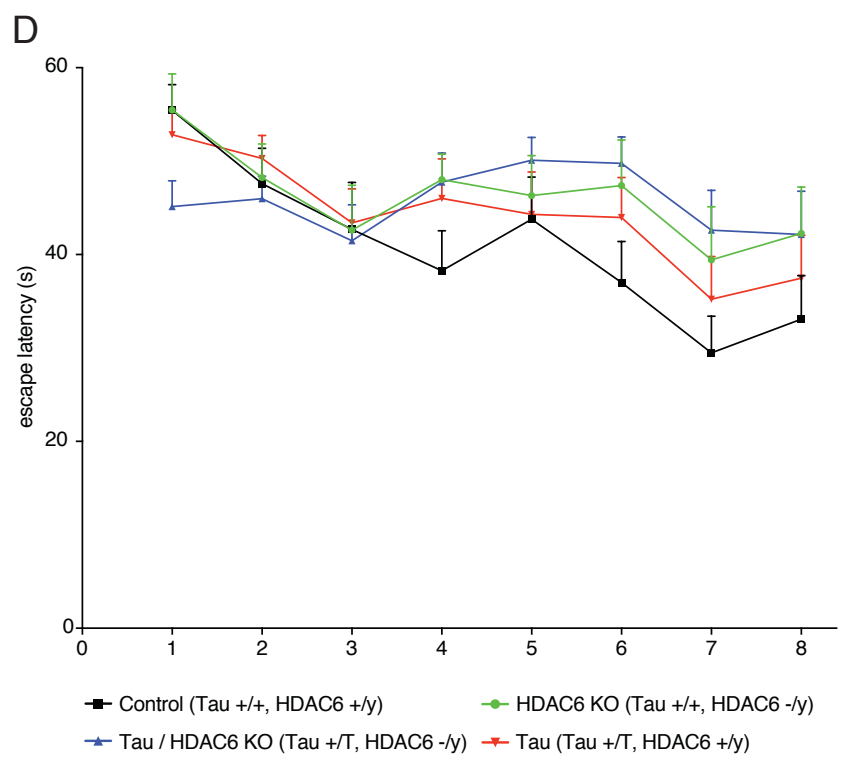

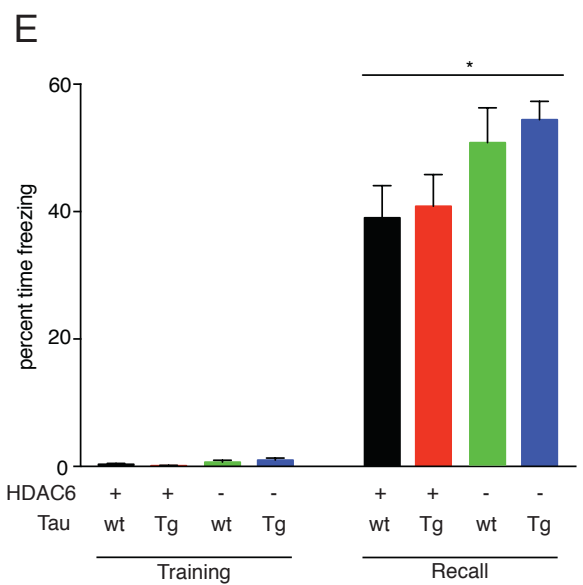

Figure 4.17: Hdac6 KO mildly modulates Tau pathology at 6 months of age (early-stage).

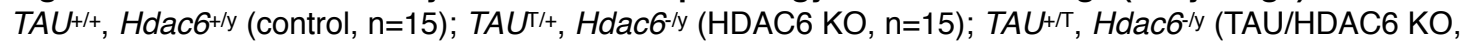
$\mathrm{n}=20$ ) or $\mathrm{TAU}^{++T}, \mathrm{Hdac6}^{+/ y}(\mathrm{Tau}, \mathrm{n}=15)$ were tested in the open field for anxiety. While no interaction between the transgenes was observed, 2-way ANOVA revealed a significantly increased time spent exposed in animals expressing the TAU transgene $(F(1,52)=10.92, p=0.0017)$. Post-hoc testing found this difference to be significant when HDAC6 KO were compared to Tau/HDAC6 KO ( $p=0.037$, Tukey) (A). Similarly travel speed was decreased in groups expressing Tau $(F(1,61)=7.14, p=0.01)$. Again posthoc testing revealed a significant difference when HDAC6 KO were compared to Tau/HDAC6 KO $(p=0.025$, Tukey) (B). Elevated plus maze did not reveal a significant difference between cohorts with no interaction of the transgenes (C). Morris water maze did not show significant differences between groups during early-stage (D). During fear conditioning all cohorts showed equal freezing. After training, during recall cohorts deficient of HDAC6 showed significantly increased freezing $F(1,63)=7.84, p=0.007)$ with no interaction with the Tau transgene. If not otherwise indicated, 2-way ANOVA was used for statistical testing, error bars indicate SEM, ${ }^{*}=p<0.05,{ }^{* *}=p<0.001,{ }^{* \star *}=p<0.0001$. 


\subsubsection{Removal of HDAC6 reinstates anxiety behaviour in aged mice}

To explore the interaction between Tau pathology and HDAC6 KO along ageing, I subjected the aged cohort (13 months of age) to the same behavioural test battery as the early-stage cohort. Late-stage groups expressing the TAU transgene displayed overall higher exposure in the open field test when compared to TAU wild-type littermates $(F(1,44)=16.48, p=0.0002$, 2-way ANOVA). Post-hoc testing revealed that this increase was only significant for Tau/HDAC6 KO when compared to control ( $p=0.002$, Tukey) and HDAC6 KO ( $p=0.003$, Tukey) but not Tau (Fig. 4.17A). Importantly, 2-way ANOVA did not report an interaction between the transgenes. To rule out involvement of motor dysfunction, I measured the travel speed during the open field and observed significantly decreased movement in cohorts expressing the TAU transgene $(F(1,43)=6.11, p=0.018$, 2-way ANOVA) (Fig. 4.17B). To asses anxious behaviour in more detail, I additionally tested all groups in the elevated plus maze and found a significant interaction between both transgenes $(F(1,39)=20.16, p<0.0001,2$-way ANOVA $)$. Post-hoc testing revealed that the Tau cohort showed a decreased preference for the closed arms when compared to control, indicating reduced anxiety ( $<<0.0001$, Tukey post-hoc), which is in line with a previous report (Van der Jeugd, Blum et al. 2013) (Fig. 4.17C). Importantly, this phenotype was reversed by HDAC6 deficiency, thus the difference between TAU wild-type and transgenic animals was absent in the Hdac6 knockout condition ( $p=0.47$, Tukey). Tau/HDAC6 KO performed similar compared to control and HDAC6 KO. Thus they spent more time in closed quadrants compared to Tau, demonstrating re-instatement of anxious behaviour.

\subsubsection{HDAC6 deficiency does not affect memory in late-stage Tau.}

I could not observe a cognitive deficit at an early-stage time point in Tau expressing groups. I went on to test spatial memory in late-stage groups. In line with the findings of the early-stage cohort, I could not identify an effect of HDAC6 deficiency in the late-stage cohort. However, I found clearly increased escape latencies based on the expression of the TAU transgene $(F(1.579,15.79)=19.09, p=0.0001$, one-way ANOVA $)$, indicative of impaired memory acquisition in TAU transgenic cohorts (Fig 4.17D). In contextual fear conditioning, I could observe a higher tendency towards initial freezing in the late-stage cohorts as compared to early-stage groups. Importantly, Tau did not show impaired behaviour during training or after conditioning when compared to control. However, similarly to the early-stage cohort, there was a trend towards increased freezing during recall trials in groups deficient of HDAC6 $(F(1,41)=6.62, p=0.014$, twoway ANOVA), indicating increased memory performance (Fig. 4.17E).

In summary, some of the findings contrast current literature on the Tau22 mouse model for ADlike tauopathy. Nonetheless, the findings emphasise the beneficial effect of HDAC6 deficiency in both age groups in contextual fear memory, where memory response is elevated above control levels. Additionally the reinstatement of anxious behaviour in this model underlines the relevance of HDAC6 modulation in the disease, particularly since an exceeding effect has been demonstrated for AD-like amyloid deposition (Govindarajan, Rao et al. 2013). 

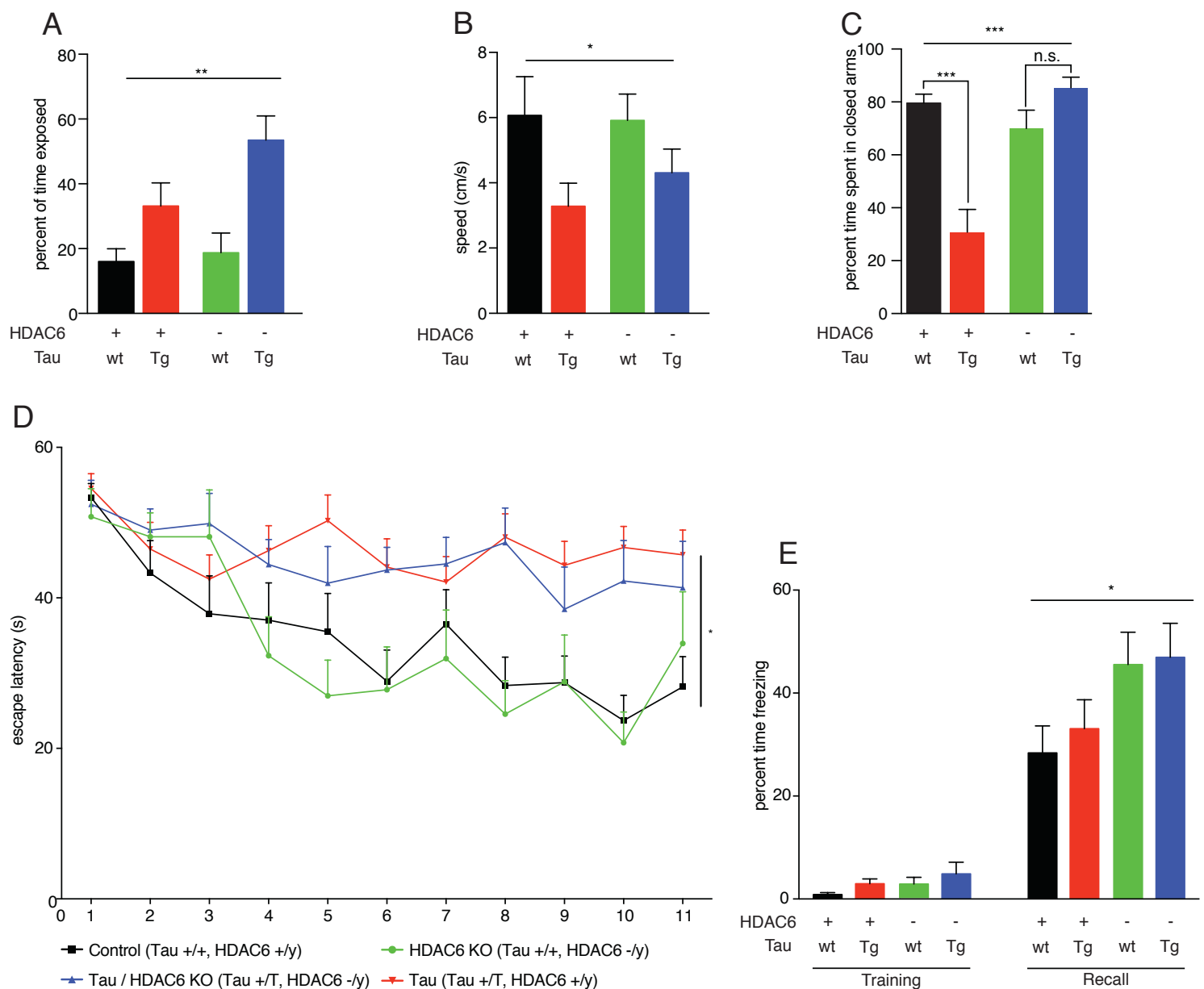

Figure 4.18: Hdac6 $\mathrm{KO}$ rescues Tau anxiety phenotype at 13 months of age.

Aged cohort of $\mathrm{TAU}^{+/+}, \mathrm{Hdac6}^{+/ y}$ (control, n=12); TAU+l+, Hdac6-/y (HDAC6 KO, n=12); TAU+/T, Hdac6-/y (TAU/HDAC6 KO, $\mathrm{n}=10$ ) or $\mathrm{TAU}^{+/ T}, \mathrm{Hdac}^{+/ y}(\mathrm{Tau}, \mathrm{n}=14)$. Open field test did not show an interaction between the transgenes $(F(1,44)=1.93, p=0.172)$, but showed increased freezing for cohorts expressing the TAU transgene $(F(1,44)=16.48, p=0.0002)(A)$. Similarly, travel speed showed no significant interaction between the transgenes $(F(1,43)=0.44, p=0.51)$, but travel speed was reduced for TAU expressing groups $(F(1,43)=6.11, p=0.018)(B)$. Percent of time spent in the closed arms of the elevated plus maze revealed a significant interaction between the transgenes $(F(1,39)=20.16, p<0.0001)$. Post-hoc testing found Tau to spent significantly less time in closed arms compared to controls $(p<0.0001$, Tukey post-hoc). This effect reversed in the absence of HDA6 ( $p<0.474$, Tukey post-hoc) (C). Morris water maze showed separation in escape latency based on presence of Tau transgene $(F(1.579,15.79)=19.09$, $\mathrm{p}=0.0001$, one-way ANOVA) (D). Contextual fear conditioning showed no significant difference between groups before training $(F(1,36)=0, p>0.999)$, however cohorts deficient of HDAC6 showed significantly increased freezing during recall $(F(1,41)=6.62, p=0.014)(E)$. If not otherwise indicated, 2-way ANOVA was used for statistical testing, error bars indicate SEM, ${ }^{*}=p<0.05,{ }^{* *}=p<0.001,{ }^{* * *}=p<0.0001$. 


\subsection{1."The diphenylpyrazol compound anle138b blocks $A \beta$ channels and rescues disease phenotypes in a mouse model for amyloid pathology"}

This Project ${ }^{1}$ resulted in a manuscript already published in EMBO Molecular Medicine on 05/12/17 (see Appendix for full article).

In this research article, we showed that oral administration of the diphenylpyrazol compound anle138b reinstated hippocampal, synaptic and transcriptional plasticity in the APP/PS1 mouse model for amyloidosis and AD. We tested 2 groups a pre-plaque group at 6 months of age and a post-plaque group at 10 months of age. Both groups were treated with anle138b or placebo for 4 months before begin of the experiments. We investigated long-term potentiation (LTP) in animals treated with anle138b before deposition of plaques or after amyloid deposition and report a full reversion of the APP/PS1 phenotype. Furthermore, we demonstrated that LTP rescue translated into a full and partial reinstatement of spatial memory in pre- and post-plaque groups respectively, in the hippocampus-dependent Morris water maze. Since AD pathogenesis correlates with deregulated gene expression we investigated transcriptome changes in both groups and found that in pre-plaque group 202 genes were differentially expressed between control and APP/PS1. Downregulated genes represented gene ontology (GO) terms associated with energy metabolism, mitochondria function, cytoskeleton integrity and synaptic plasticity, while categories linked to cell growth were enriched by unregulated genes. Importantly, when control and anle138b-treated APP/PS1 of the pre-plaque group were compared, only 27 genes remained differentially regulated and no significantly enriched pathways could be identified. Thus the APP/PS1 transcriptional phenotype was almost fully reversed by anle138b in the preplaque group.

In the post-plaque group, the APP/PS1 placebo-treated cohort showed 130 genes differentially regulated when compared to controls. These genes were also deregulated in APP/PS1 anle138b-treated when compared to controls and an additional 117 genes were differentially expressed which were linked to induction of inflammatory responses. This indicated that the reinstatement of the observed phenotypes with anle138b treatment was not based on reduction of amyloid induced inflammation. To obtain mechanistic insight, we characterised membrane conductivity in the presence of anle138b using black lipid membranes (BLM). This work was conducted in collaboration with Gillmann and Lee. We showed that $A \beta_{1-40}$ treatment of DOPS/ POPE (1:1) membranes induced stepwise growth of membrane conductance (Fig 4.18A). Importantly, anle138b doped membranes showed fluctuation but no stepwise increase and reduced bulk conductance (Fig 4.18B). These results indicated that anle138b treatment alters pore dynamics, resulting in short lived and less stable available pores.

To sustain the notion that anle138b alters $A \beta_{1-40}$ pore properties in vivo, I established a protocol which allowed me to treat primary hippocampal neurons with $A \beta_{1-40}$ monomers or oligomers and

\footnotetext{
1 This project was executed in cooperation with Ana Martinez Hernandez (coordinated the experiments, performed behaviour testing of mice, RNAseq and amyloid plaque analysis), Alan L. Gillman (performed electrophysiology on black membranes), and Joon Lee (AFM measurements of $A \beta$ on membranes)
} 
asses membrane permeability and cell viability (Fig 4.18C). Using this protocol, I showed that the addition of $A \beta_{1-40}$ oligomers but not $A \beta_{1-40}$ monomers significantly decreased fluorescence, indicating reduced membrane selectivity as measured by using the CyQuant cell permeability assay (Fig 4.18D). Importantly, this effect was reversed when neurons were pre-treated with anle138b $24 \mathrm{~h}$ before the addition of $\mathrm{A} \beta$ (Fig 4.18D). In order to show that this restoration was not due to an anle138b mediated reduction of cell death, I tested the viability of hippocampal neurons in the presence and absence of anle138b, using the metabolism based MTT assay. The hippocampal neuronal viability was equally affected by $A \beta_{1-40}$ monomer and oligomer treatment in my experimental setup (Fig 4.18E). To test if the effect on membrane integrity depended on the pre-incubation with anle138b, I reversed the application of $A \beta$ species and anle138b (Fig. 4.18F), analogous to the post-plaque treatment in vivo. Also in this case did the $A \beta$ oligomer but not monomer exhibit a negative effect on membrane integrity. Application of anle138b was also able to suppress the effect of toxic $A \beta$ oligomers on membrane integrity, when the experimental procedure was reversed and anle138b was supplemented after hippocampal neurons had been incubated with $A \beta$ oligomers for $24 \mathrm{~h}$ (Fig 4.18G). At this time point neurons had already shown reduced viability (Fig $4.18 \mathrm{H}$ ). To a certain extent, this scenario resembled that of the post-plaque group in that neuronal damage preceded anle138b treatment. These results were in line with our data demonstrating that anle138b reversed LTP and partially restored memory deficits in the post-plaque group. In summary, we demonstrate that anle138b treatment reverses key pathological hallmarks in a model for $A B$ pathology before and after onset of the pathology. These findings are in line with previous beneficial reports in Tau pathology models and underline the clinical relevance of anle138b to treat both molecular pathologies of $A D$.

Figure 4.19: Anle138b ameliorates $A \beta_{1-40}$ induced membrane permeability.

Application of $A \beta_{1-40}$ to black lipid membrane induced stepwise increase in conductance. This increase followed the direction of current, thus inverted when the applied voltage was reversed (top), while application of anle138b abolished increase beyond 10 pA (bottom) (courtesy of Gillman and Lee, cf. text) (A). Detail of anle138b doped trace from (A) discreet conductance levels (dashed, blue) suggested events for three individual pores (B). On DIV 10 hippocampal neurons were treated with anle138b (1 $\mu \mathrm{M})$ or vehicle $24 \mathrm{~h}$ prior to $\mathrm{A} \beta_{1-40}$ mono or oligomers (10 $\mu \mathrm{M}, \mathrm{n}=4 /$ group) application. Fluorescence was measured as indicator for membrane integrity $48 \mathrm{~h}$ after $A \beta$ application using a CyQUANT assay (Thermo Fisher) (C). Vehicle treated neurons showed significantly impaired membrane integrity after $A \beta$ oligomer application compared to control ( $p=0.007$, t-test) or monomer treatment. Importantly, membrane integrity was rescued when anle138b was applied beforehand (D). Same experimental conditions as (D) but MTT assay was used to measure cell viability. $A \beta$ treatment (mono- and oligomer) lead to reduced viability compared to control in $(p=0.002$, t-test) regardless of anle138b $(E)$. The experiment was conducted as described in $(C)$ but with reversed application of anle138b and $A \beta_{1-40}$ species (F). A significant group difference was revealed by one-way ANOVA. Tukey post-hoc testing found significantly reduced membrane integrity application of $A \beta_{1-40}$ oligomers without anle138b ( $p=0.003$ vehicle: control vs. $A \beta$ oligomers). Importantly, treatment with anle138b after oligomer application reversed this effect (G). Cell viability was assessed using MTT assay and same experimental approach as (G). A $\beta_{1-40}$ oligomers reduced cell viability in the presence and absence of anle138b (Group difference $p<0.5$, Oneway ANOVA with post-hoc testing) $(\mathbf{H})$. Error bars indicate SEM. 

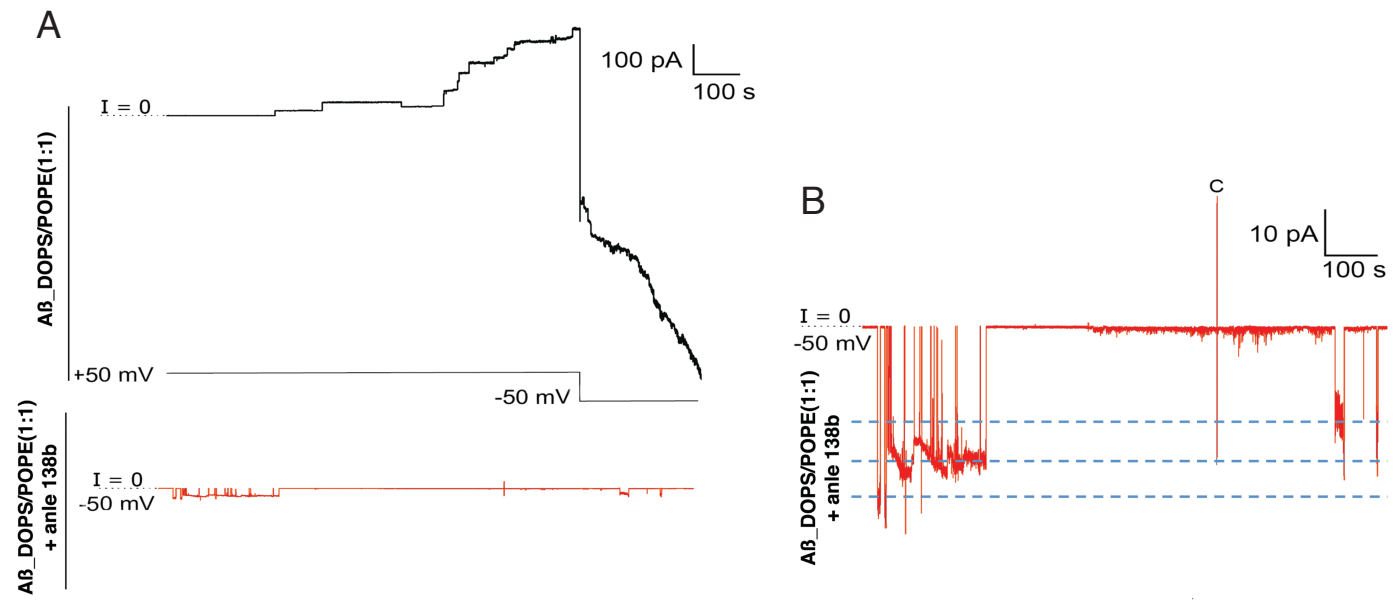

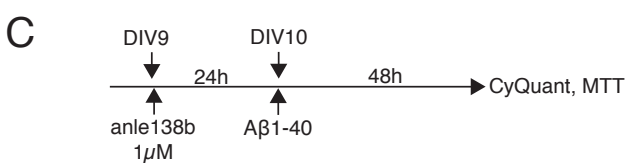

D

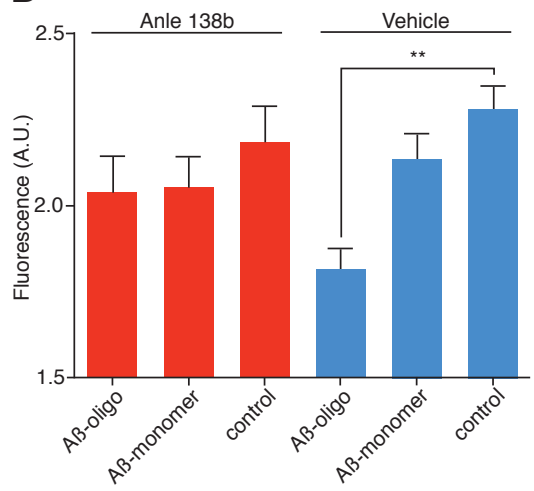

E

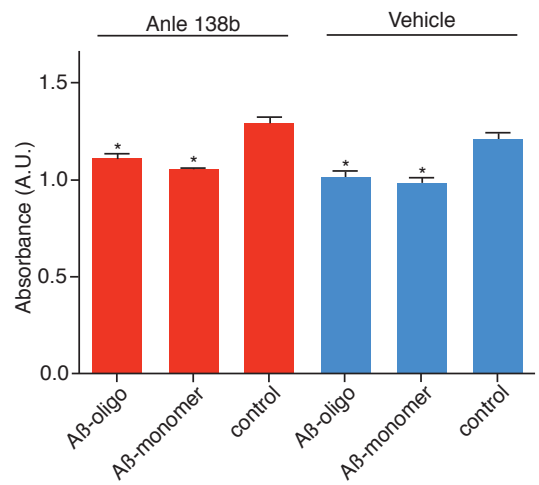

F

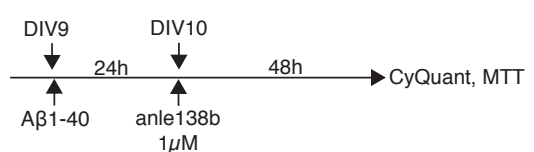

G

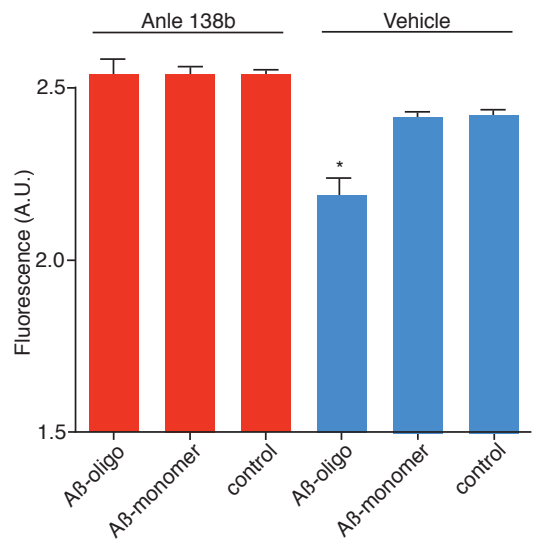

$\mathrm{H}$

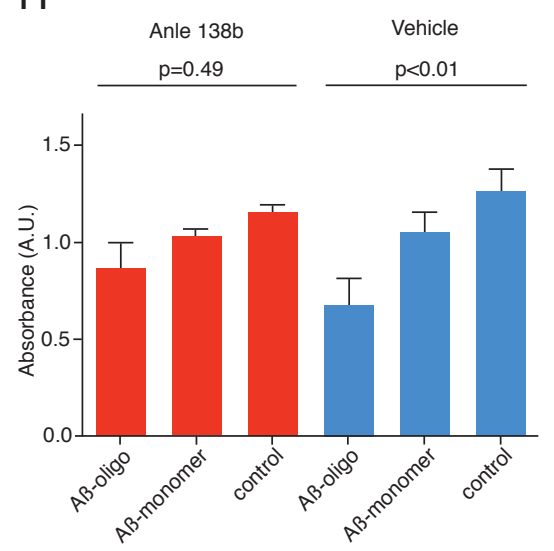




\subsection{Analysing neuronal network dynamics in primary hippocampal cultures}

Little is known about the interplay of cells within a neuronal network which is active during a stimulus. It is unknown whether neurons that are active differ in their transcriptional state compared to those secondarily activated by the initially stimulated cells. To be able to distinguish the populations fluorescent tags and genetic tools need to be introduced into subsets of the network. Thus, I established a new method to transmit genetic modification into defined subsets of neuronal cells independent of promoter usage or genotype using AAV deliverable constructs. Optogenetic tools offer high spatial and temporal specificity, to introduce stimulation into the network. However, available devices for optogenetic stimulation did not meet the requirements. Thus, I designed and built the Luminous Channelrhodopsin Inductor For Research - LuCIFR.

\subsubsection{Efficient composition of neuronal networks with multiple mutations}

In order to build neuronal networks in which defined percentages, down to single cells, harbour a desired modification, I turned to adeno-associated viruses (AAVs). They are highly efficient viral particles with near absolute infection efficiency in culture and can deliver genetic constructs of up to $4.5 \mathrm{~kb}$. This is sufficient for most cargos including protein overexpression, fluorescence tagging, CRISPR/Cas9, or Optogenetic tools. In brief, the construct delivery protocol I developed requires freshly dissociated primary hippocampal neurons, which are resuspended in medium containing a $\mathrm{CO}_{2}$ resource. $\mathrm{AAV}$ s are then added to the suspension at high titer and incubated for $1 \mathrm{~h}$ at $37^{\circ} \mathrm{C}$ after which cells are seeded at the desired concentration (Fig. 4.19A, cf. "3.4.4. Heterogenous primary neuronal cultures"). The construct delivery efficiency is dependent on viral titer and scalable by increasing the infectious units per reaction (Fig. 4.19B). Notably, high titer infections require presence of heparin at $5 \mu \mathrm{g} / \mu \mathrm{l}$ in the maintenance medium to absorb AAVs released from cellular debris. Under my supervision, Lukas Faiß² used this protocol during his B.Sc. thesis to deliver Channelrhodopsin2 (ChR2 H134R) to a distinct subpopulations of neurons in a culture. He found Photo-stimulation of neuronal cultures with $10 \%$ ChR positive $\left(\mathrm{ChR}^{+}\right)$neurons showed increase in cFOS protein levels 30 min after activation in

Figure 4.20: Production of heterogeneous neuronal cultures.

Neuronal cells were resuspended in infection medium, supplemented with AAV and seeded at desired ratios (A). Illustration of heterogeneous cultures with two subpopulations, one expressing GFP and another expressing mCherry after infection at low titer (top) and high titer (bottom) AAV (B). Heterogeneous culture with $10 \% \mathrm{ChR}^{+}(\mathrm{H} 134 \mathrm{R})$ neurons. After light stimulation cFos was induced in surrounding but not $\mathrm{ChR2}+(\mathrm{H} 134 \mathrm{R})$ cells as indicated by arrows (top). No induction was visible in the absence of ChR (bottom) (C). FACSorting of heterogenous cultures seeded at 1:9 (ChIEF-tdTomato:FoseYFP-reporter) shows $13.8 \%$ and $88.3 \%$ sorted from parent population (D). 3:7 (ChIEF-tdTomato:FoseYFP-reporter) seeding ratio results in $27.2 \%$ and $76.1 \%$ sorted cells from parent population (E). Cell purity after FACS in sample of 20 sorted cells (F) and single sorted cells (G) for ChIEF-tdTomato and Fos-eYFP-reporter. Experiments for (C) were performed by Lukas Faiß under my supervision.

2 Driving transcriptome changes in a neuronal network - a new model, Georg-August-Universität, Göttingen 
A

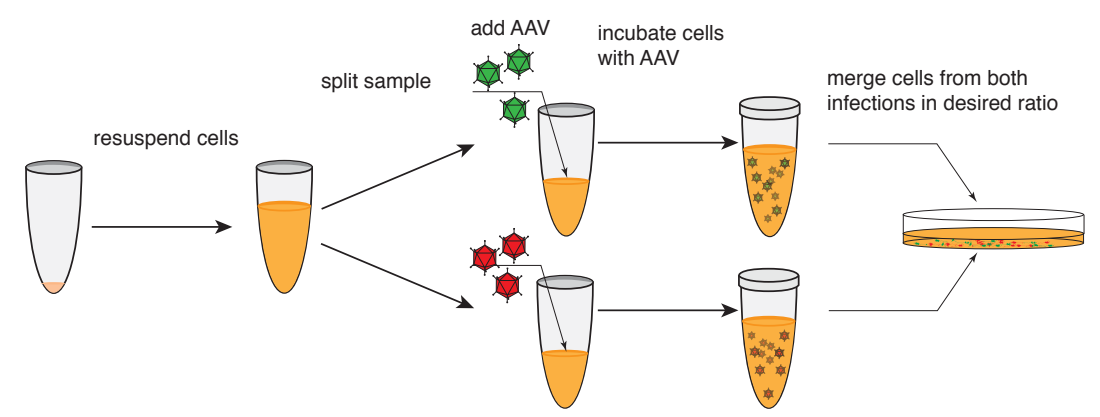

B
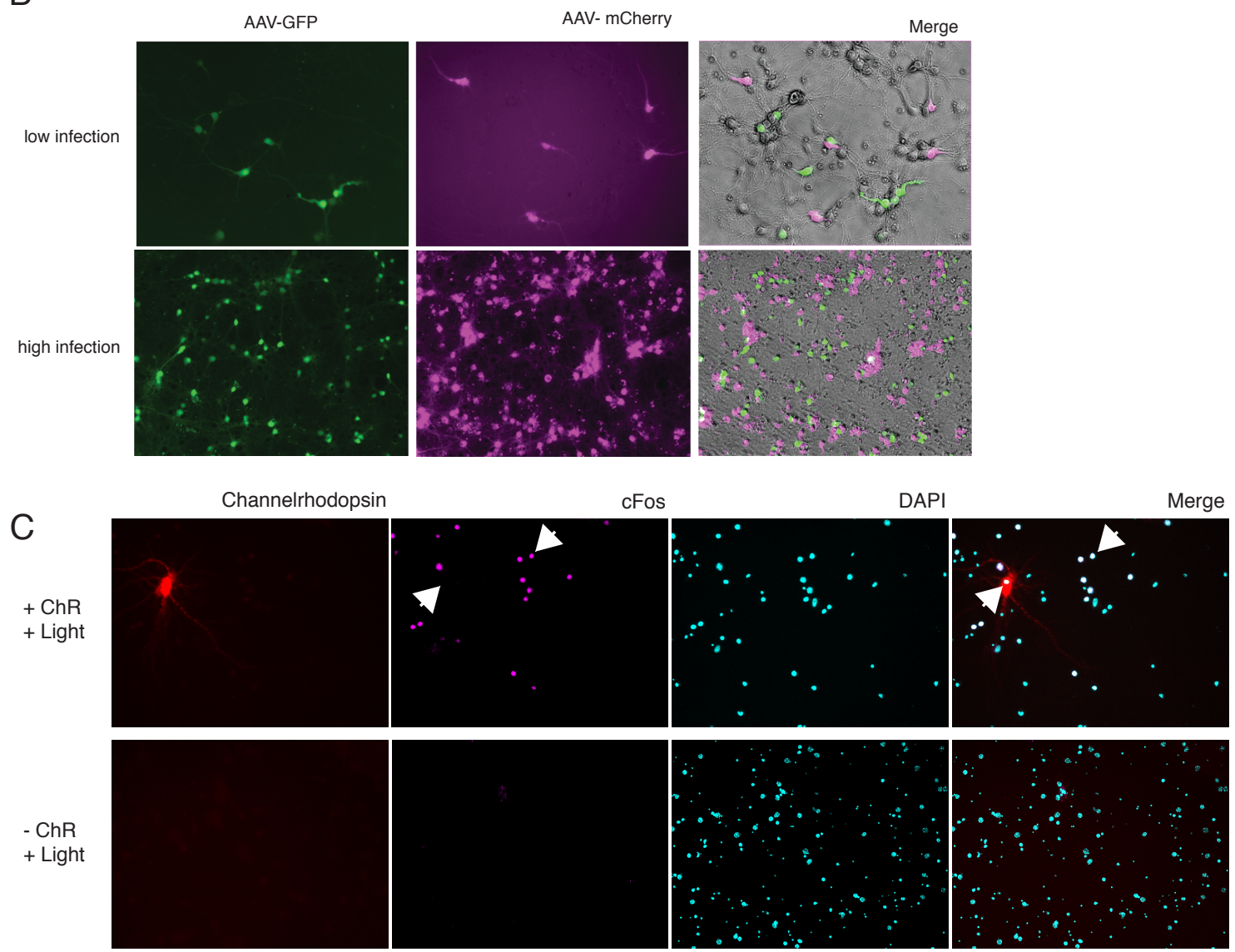

D

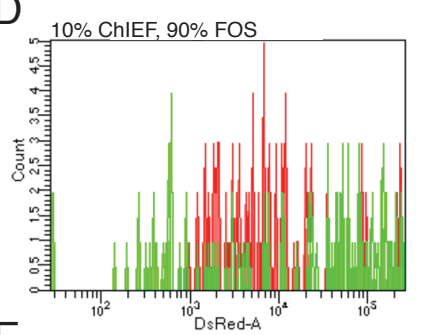

E

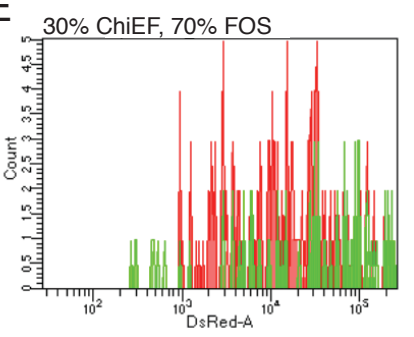

F ChlEF-td-Tomato

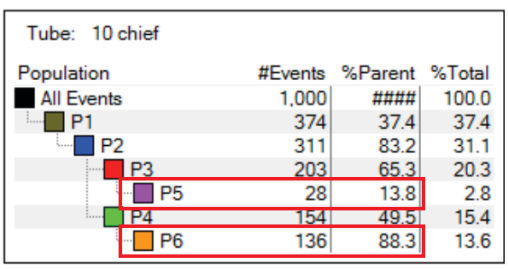

G

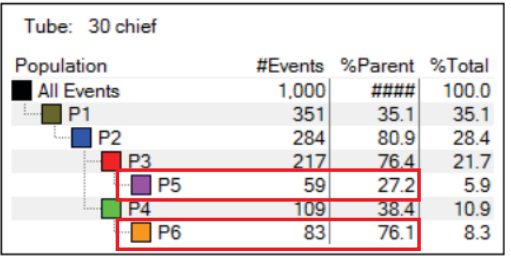

Fos-eYFP reporter
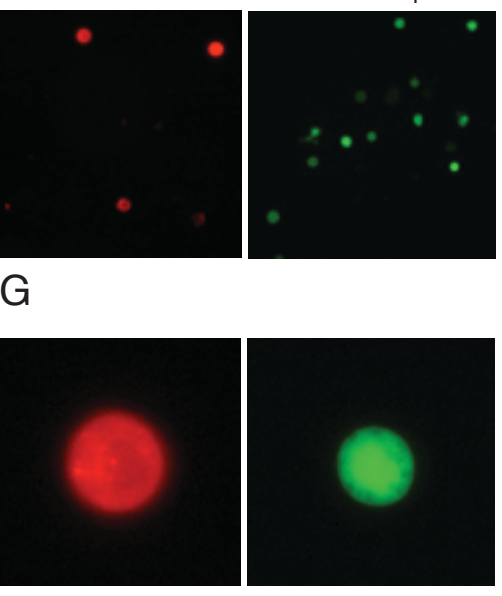
secondarily stimulated cells but not primarily stimulated ChR2+ cells (Fig. 4.19C). To follow up on this intriguing finding I established another protocol to be able to separate cells that expressed Channelrhodopsin and cells that were activated secondarily by $\mathrm{ChR}^{+}$photo-activated cells. To this end, I first switched from ChR2H134R to the faster and more sensitive ChIEF variant fused to tdTomato and introduced an activity reporter using Fos promoter-driven eYFP expression. The two constructs were delivered to separate subpopulations of the same PNC at a ChIEF+ to FOS-reporter+ cell ratio of 1:100, 1:9, 3:7 and 1:1. On DIV10 neurons were photostimulated and sorted according to their fluorescence tag using FACS. With the newly established protocol I was able to achieve high sorting purity of intact cells from heterogeneous cultures. At a seeding ratio of 1:9 FACS showed a ratio $13.8 \%$ and $88.3 \%$ (Fig. 4.19D); for a seeding ratio of $3: 7$ FACS resulted in $27.2 \%$ and $76.1 \%$ of parental population (Fig. 4.19E). Sorting efficiency was assed using fluorescence microscopy for a pool of $20 \mathrm{ChIEF}^{+}$and FOSreporter ${ }^{+}$cells each (Fig. 4.19F) and showed no single false positive. Single cell sorting into 96 well plates gave identical results in purity and accuracy (Fig. 4.19G). To investigate molecular properties of primary and secondarily stimulated cells I sorted 384 cells according to the established protocols and together with the LIMES-institute in Bonn subjected them to Massively Parallel Single-Cell RNA-Seq (MARSeq) (Jaitin, Kenigsberg et al. 2014) . The results of this data however are pending at the time of writing this thesis.

\subsubsection{Introducing the Luminous Channelrhodopsin Inducer For Research (LuCIFR)}

Current commercial solutions for light-induced activation of cells in culture using optogenetics lack several key aspects relevant to molecular experiments which are conducted in $\mathrm{CO} 2$ incubators. Self-made solutions often trade accuracy for affordability or are too complex to be built in standard research facilities. Thus, I designed a new solution targeting specifically the needs of molecular biologists working with live cells in incubators. A fundamental difference in the approach is that LuCIFR is engineered to give high precision while maintaining easy access to the user at an affordable price. At the time of writing LuCIFR is licensed to industrial partners, thus I will focus on prototypes, as the exact blueprints of LuCIFR are classified (EUIPO\#: 004093268).

Prototype A featured 6 high power LEDs in a plastic enclosure controlled by an H-bridge receiving input from an Arduino UNO micro-controller and powered via USB (Arduino) and a constant current source (LED/H-bridge) (Fig. 4.20A). The second iteration (prototype B) was upgraded with a bluetooth module for wireless communication (Fig 4.20A+B). Custom-written software was used to receive and transmit stimulation protocols from the operator via bluetooth. For stimulation blue LEDs at $475 \mathrm{~nm}$ were used to cover the peak absorption spectrum of Channelrhodopsin (ChR2H134R) (Fig. 4.20D). The LED was setup in a way that only the top $80^{\circ}$ angle was used per well to avoid edge intensity drop off, and the LED was placed in 2.08 $\mathrm{cm}$ distance to the dish. This setup resulted in a stimulation intensity $0.057 \mathrm{Im} / \mathrm{mm}^{2}$ which in turn 
allowed the incorporation of a dispersion filter due to high photo current (Fig. 4.20C). Stimulation efficiency was electrophysiologically tested by patch clamping (courtesy of Markus Stahlberg). Test stimulation of ChR2H134R expressing primary neurons using prototype A shows high responsiveness of the cells to light stimulus during a single pulse $2 \mathrm{~Hz}$ stimulation and $20 \mathrm{~Hz}$ stimulation. At $25 \mathrm{~Hz}$ long term stimulation ChR2H134R reaction showed a plateau due to channel recovery latency (Fig. 4.20E), while prototype A stimulation frequency exceeds $16 \mathrm{MHz}$ with $25 \mathrm{~ns}$ pulse delay. Stimulation efficiency was also tested by qPCR with cFos mRNA expression levels as read-out (courtesy of Lukas Faiß). Notably, cFos was increased slightly $15 \mathrm{~min}$ post stimulation and significantly $30 \mathrm{~min}$ after stimulation. For prototype B I received the "Innovation to application award" which allowed the further development of the hardware and to build a waterproof enclosure together with A3M (engineer, Braunschweig) and Neumanns Büro (industrial designer, Braunschweig) (Fig 4.20G-L).

Figure 4.21: LuCIFR a new optogenetic stimulator for molecular biology.

Prototype-A showing breadboard micro controller, constant current source, high Power LED cluster in waterproof enclosure (A). Basic circuit of prototype-B shows bluetooth module upgrade and change from $\mathrm{H}$-bridge to Tip120 transistor control (B). Illustration of LED \% luminous flux spectrum coverage and $\mathrm{ChR2}(\mathrm{H} 134 \mathrm{R})$ absorption spectrum shows peak at activation peak (D). Only $80 \%$ of total emission angle are used for direct illumination (dark blue), rest is scattered (light blue) (D, top). LED is positioned central below well with $2.08 \mathrm{~cm}$ distance to dispersion filter ( $D$, bottom). Patch clamping (performed by Markus Stahlberg) shows reactivity of $\mathrm{ChR} 2^{+}$primary neurons to prototype-B stimulation for single pulse, $2 \mathrm{~Hz}$, $20 \mathrm{~Hz}$, and plateaus at $25 \mathrm{~Hz}(E)$. cFos is increased in qPCR $15 \mathrm{~min}$ and significant $30 \mathrm{~min}$ after Illumination with prototype-B (qPCR performed by Lukas Faiß) (F). Illustration of LuCIFR after further development supported by the "innovation to application" award in waterproof enclosure, illumination area (white) and docking station (G-L). 

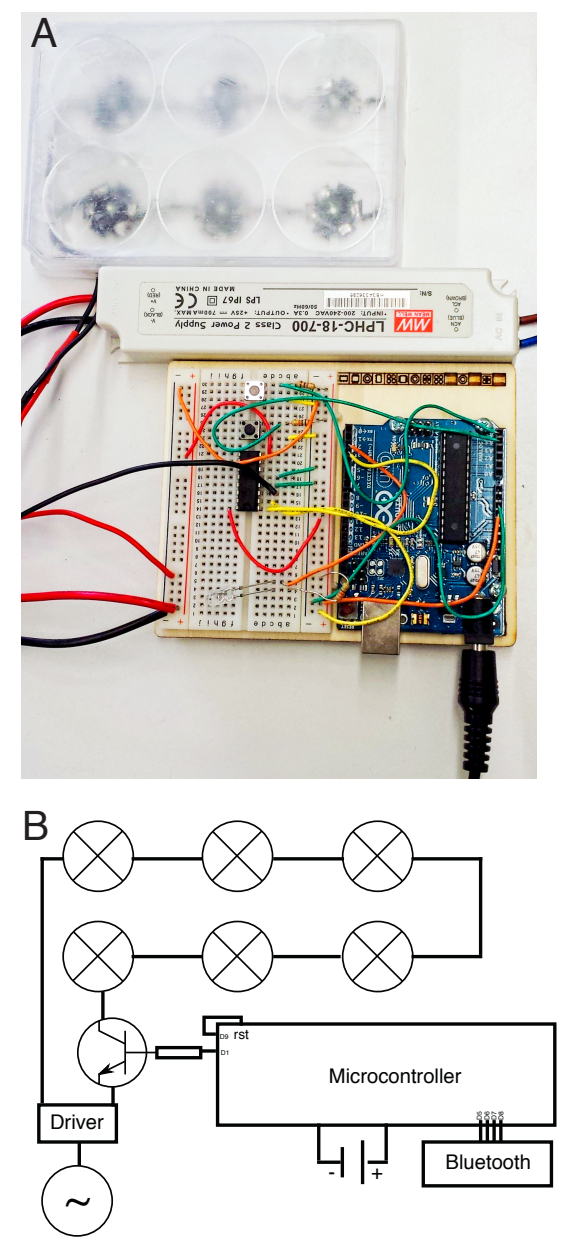

C

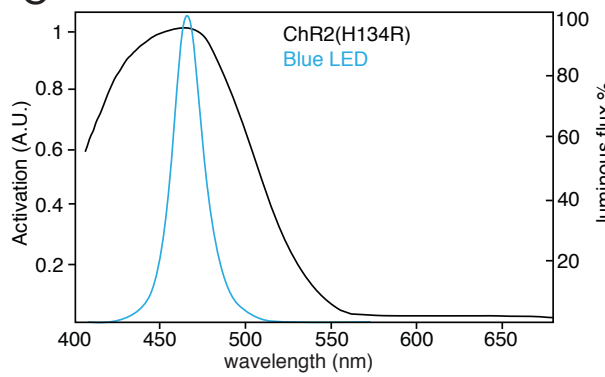

G
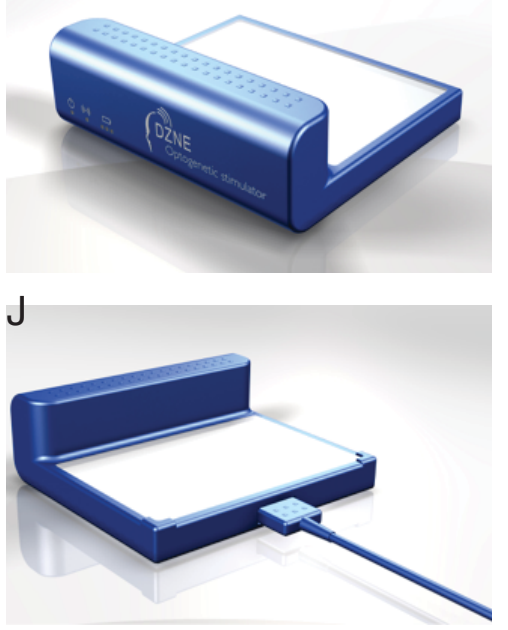

K
D
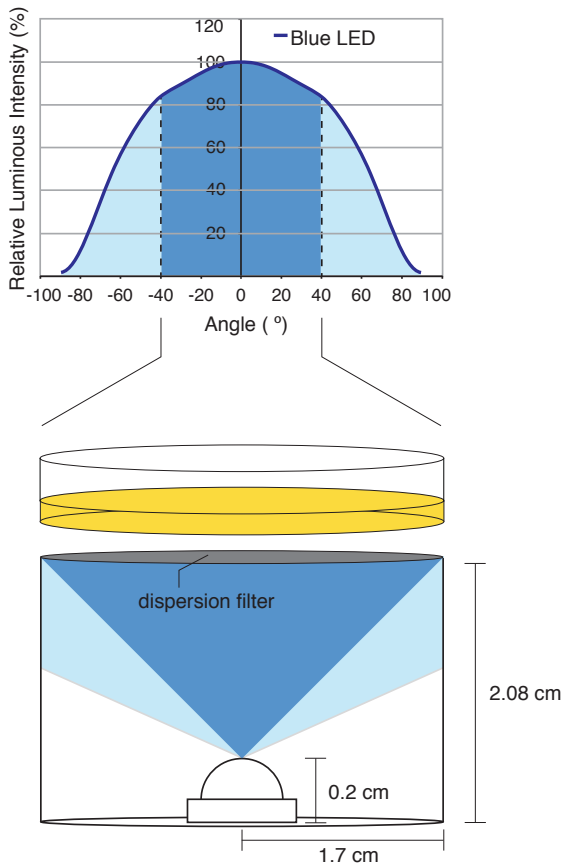

F

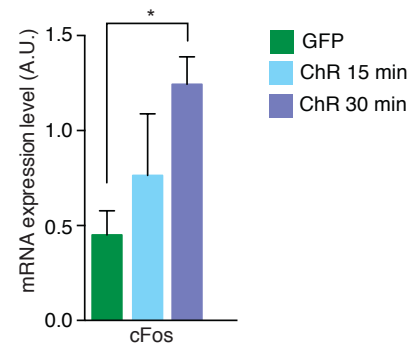

E
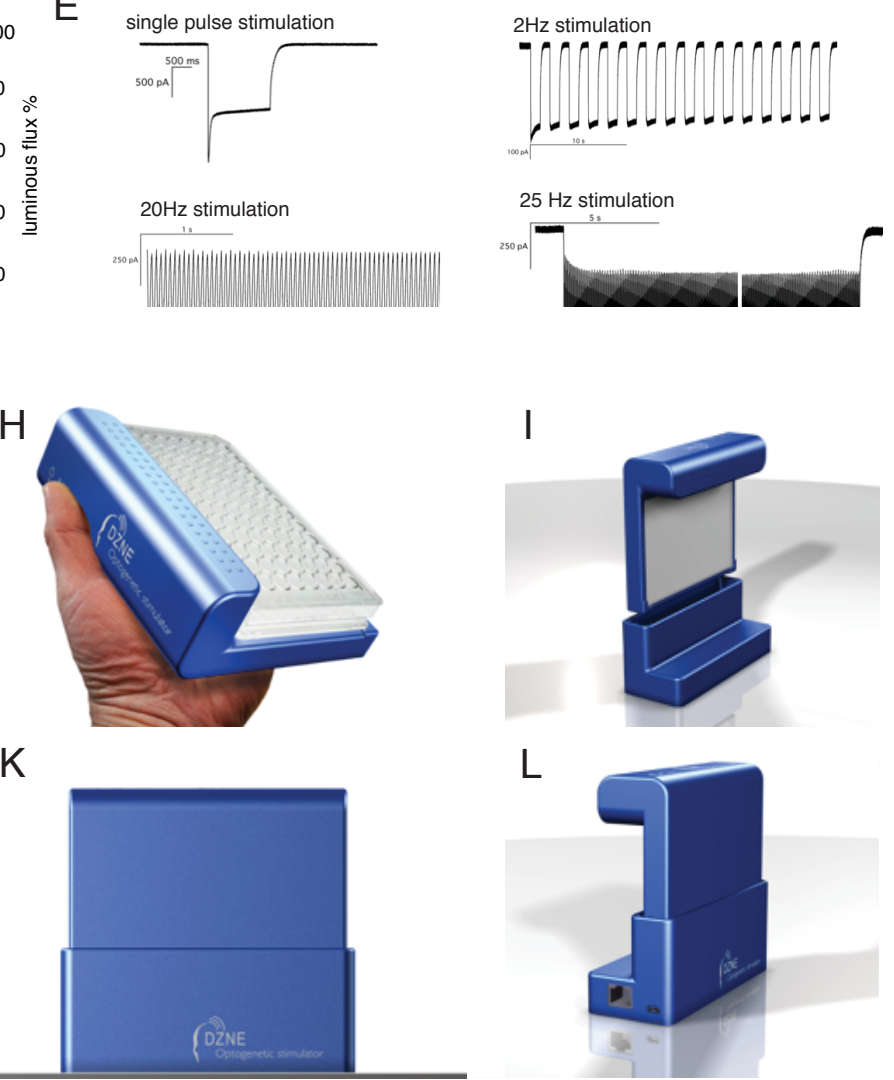

L

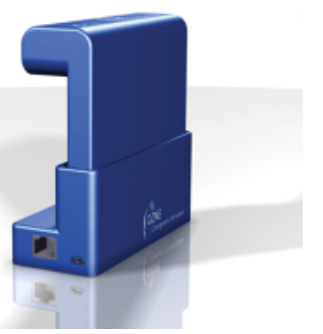




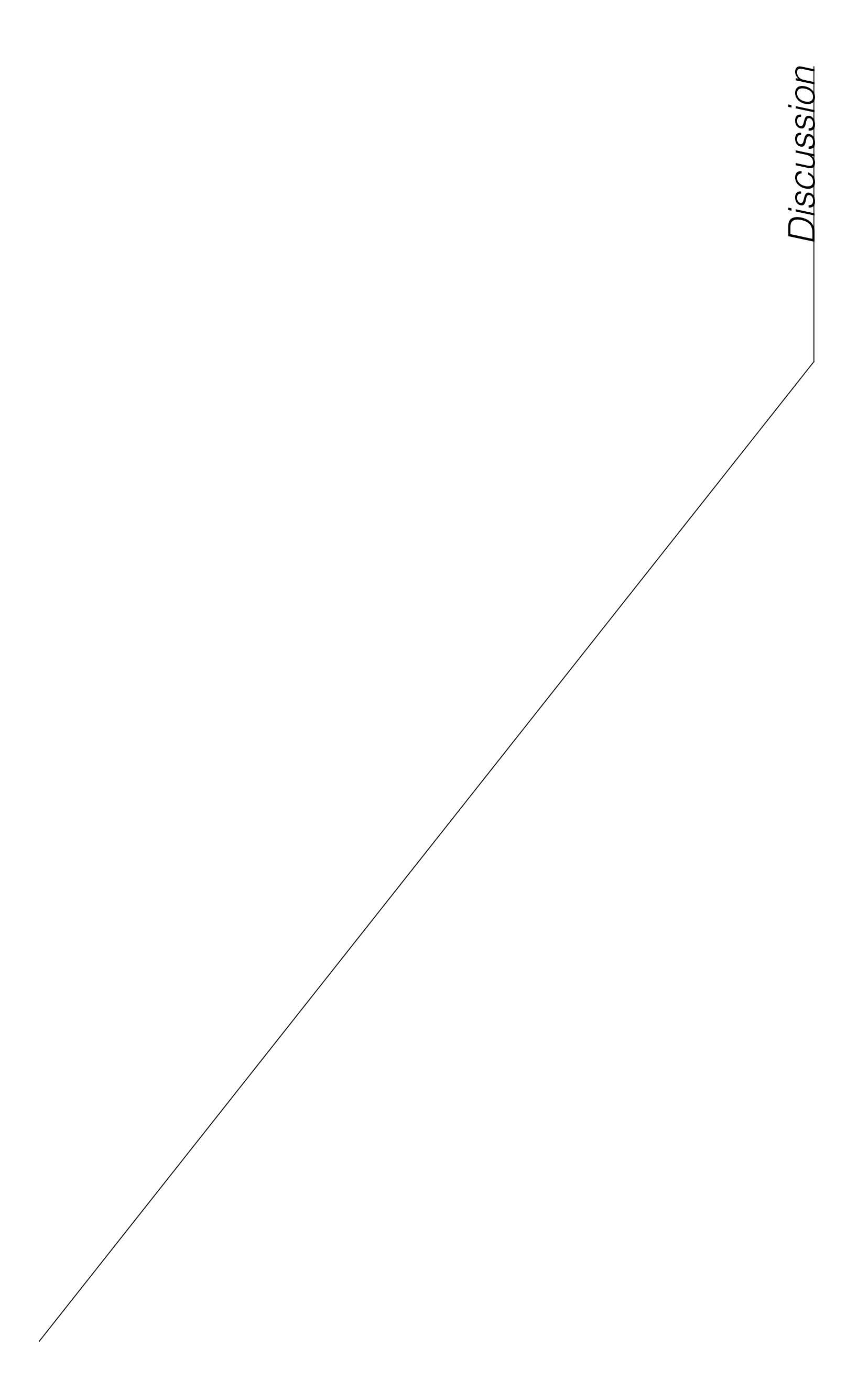




\section{Discussion}

Alzheimer's disease (AD) is a multifactorial disease with detrimental social and economic impact. The number of patients affected is expected to almost triple by the year 2050, increasing socio-economic pressure on relatives and society (World Alzheimer Report 2016). Despite tremendous efforts, to date there is no cure and no efficient treatment available. It is commonly accepted that the appearance and progression of $A D$ depend on the interplay between genetic risk factors and environmental influence. The epigenetic machinery is exactly at this interface. To this end, I dedicated significant efforts to study novel players of the epigenetic machinery that might serve as new therapeutic targets in cognitive deficits and in AD. I identified ANXA2 as a novel H4K12ac chromatin reader in neurons and went on to characterise its involvement in learning and memory. My observations suggest that ANXA2 is a baseline inhibitor of spatial memory function and that deleting it could be a suitable strategy to enhance cognitive performance. Mechanistically the data suggests that ANXA2 influences two key aspects relevant to learning and memory. Firstly, ANXA2 contributes to extracellular matrix stability, which is known to be a regulator of synaptic plasticity. Secondly, ANXA2 regulates genes relevant for energy metabolism through alternative 5' usage. Although I did not specifically test the effect of ANXA2 deletion/inhibition in AD models, my data suggest that this could be a suitable strategy. On the other hand, I dedicated part of my work to explore novel therapeutic targets with the potential to become therapies against AD. I used both a pharmacological approach (anle138b and JS28) and a genetic model of HDAC6 deletion (Tau/ HDAC6). Both strategies reveal promising beneficial effects in different models for AD.

In addition to studying concrete aspects of AD pathophysiology and epigenetic regulators of cognition, I also developed a novel technical strategy to study network dynamics in neurons. First I designed and optimised a novel AAV-based vector system to target individual neuronal populations. I demonstrated the in vitro effectiveness of this system with a proof-of-principle experiment involving channelrhodopsin-mediated activation of neuronal subpopulations. Importantly, this system is highly customisable and could be easily adapted for other types of applications. As a complementary approach, I designed and engineered a novel device for optogenetic stimulation in cell culture (LuCIFR). By combining the vector-based subpopulation targeting with FACS sorting, single-cell sequencing and the novel optogenetic device, I came up with a revolutionary technical approach to study network dynamics and individual population contributions to different kinds of processes, including activity- and network-dependent transcriptomic profiles.

Thus, I used 3 complementary approaches to study molecular pathways in cognitive processes with an emphasis on therapeutic applications. I established a role for ANXA2 as a chromatin reader involved in spatial memory, contributed to the characterisation of 2 highly novel $A D$ treatment strategies (HDAC6 deletion and anle138b), and developed a technical strategy to study neuronal network dynamics. Although each of these approaches bears different 
implications for further research and will have to be pursued separately, collectively they constitute a significant advance in the field of molecular neurocognitive research and have great potential for further development. In the following sections, I will discuss each of the approaches separately with an emphasis on their significance in the context of the current state of research and their potential implications.

\subsection{ANXA2- a novel reader of $\mathrm{H} 4 \mathrm{~K} 12 \mathrm{ac}$}

Alzheimer's Disease (AD) and many other neurological disorders develop from the interplay between genetic and environmental factors. It is therefore highly relevant to study proteins that mediate this cross-talk between the genome and the environment. There are newly emerged key players of the epigenetic machinery, namely chromatin readers, that are becoming more and more relevant for our understanding of disease mechanisms and therapeutic strategy development. I identified and characterised ANXA2, a novel chromatin reader of acetylated H4K12. Importantly, I demonstrated for the first time that ANXA2 is a baseline inhibitor of spatial memory and that genetically inhibiting it could have a beneficial effect on memory that recapitulates the effects of HDAC inhibitors (Guan, Haggarty et al. 2009, Stefanko, Barrett et al. 2009, reviewed in Fischer, Sananbenesi et al. 2010, Benito, Urbanke et al. 2015). This puts readers into focus as new drug targets. Importantly, chromatin reader inhibitors to date have been reported to be much more specific as compared to HDAC inhibitors (Korb, Herre et al. 2015, Magistri, Velmeshev et al. 2016, Benito, Ramachandran et al. 2017). Thus a targeted drug to ANXA2 could be applied to treat dementia symptomatically, as it would not target a causal factor but circumvent it through transcriptional adaptation. Such a drug would be beneficial not only in $A D$ but also in age-associated memory impairment and further dementia associated diseases.

\subsubsection{ANXA2 reads the histone code in the mouse brain}

Peleg and colleges demonstrated that defective learning-associated acetylation of lysine 12 on Histone 4 (H4K12ac) correlates to age-associated cognitive impairment (Peleg, Sananbenesi et al. 2010). Intrigued by this finding, I wanted to understand how this specific post-translational modification (PTM) of a histone tail could be translated into transcriptomic changes and more specifically how this PTM can play a major role in cognitive decline in the framework of new epigenetic players. The prevalent model for how PTMs, and acetylation in particular, influence transcription is that histone tails are dynamically modified by writers (e.g., HATs) and erasers (e.g., HDACs), induce conformational changes and would make the DNA more or less accessible to the transcriptional machinery (Strahl and Allis 2000). Further, Strahl and Allis proposed that proteins without own enzymatic capabilities could recognise specific PTMs and serve as a scaffold for larger complexes involved in transcriptional regulation. These so-called "chromatin readers" are capable of binding individual or combinatorial patterns of PTMs at 
histone tails and thus "translate" the histone code by turning epigenetic information into highly orchestrated transcriptional patterns. Obviously, this model can rise to high complexity, making it very relevant to identify entities that have an influence on neuroepigenetic mechanisms. Based on the evidence that H4K12ac is a very important mark for cognitive function, in particular in age-associated memory decline, I hypothesised that a chromatin reader of this PTM would itself be relevant to learning and memory and contribute to the understanding how a PTM is translated into a cognitive phenotype. To identify new targets, Eva Benito and I used stable isotope labelling with amino acids (SILAC) to produce protein lysates of soluble chromatin (Spellman, Deinhardt et al. 2008). These lysates were exposed to a H4K12ac or H4K12 mimic as a bait to capture peptides associating with the mark. This was followed by a pulldown and LC-MS/MS to identify bound peptides (collaboration with Nadin Zimmermann) (Fig. 4.1A-C). Using this approach, any peptide showing a significant preference for the acetylated compared to unmodified H4K12 was a highly interesting target. Notably, the screening revealed four proteins significantly binding the mark, one of which was BRD4. BRD4 is a member of the BET (Bromodomain and extra-terminal domain) family of proteins and one of the best studied chromatin readers in the brain (Korb, Herre et al. 2015, Sartor, Powell et al. 2015, Li, Ma et al. 2016, Benito, Ramachandran et al. 2017). This finding was highly encouraging for our approach, especially because BRD4 displayed the lowest affinity to the mark compared to the other identified chromatin readers. This entails that the other targets we identified are potentially even more interesting from a functional perspective. While this is speculative, the increased selectivity to H4K12ac could indicate a more stable scaffold or higher impact on the adjacent genomic region.

In this unbiased screen, we identified ANXA2, which not only showed the second highest affinity to acetylated H4K12, but also happens to be a protein with extensively studied functions in the cytosolic compartment. ANXA2 implicated with tumour progression (Wang and Lin 2014) and activation of STAT6 (Das, Shetty et al. 2010). It was also associated with frontotemporal dementia (Gauthier-Kemper, Weissmann et al. 2011, Hallmann, Arauzo-Bravo et al. 2017) and was shown to change sub-cellular location during spatial learning (Zhao, Waisman et al. 2004). Furthermore, it was reported to be able to shuttle from the membrane to the nucleus (Liu and Vishwanatha 2007), bind and repair DNA (Boyko, Mudrak et al. 1994, Madureira, Hill et al. 2012), RNA (Filipenko, MacLeod et al. 2004), recognise primers as a cofactor for DNA polymerase a (Kumble, Iversen et al. 1992, Vishwanatha, Jindal et al. 1992) and was implicated with the SMARCA3 chromatin remodelling complex in neurons (Oh, Gao et al. 2013). Taken together, theses findings suggest a widespread role of ANXA2 in cellular functions, but the role as a chromatin reader specifically is to the best of my knowledge novel and has not been reported before. Considering the previously reported functions of ANXA2 associating with genomic functions such as transcription, primer recognition, splicing and chromatin structures could potentially be linked to the common function as a chromatin reader. 
Mapping ANXA2 in the mouse brain showed that ANXA2 is ubiquitously expressed in brain areas associated with higher cognitive function. Moreover, the protein was found in every subcellular compartment tested from membrane to cytosol and nucleus, which is in line with earlier reports (Raynal and Pollard 1994, Deora, Kreitzer et al. 2004, Zheng, Foley et al. 2011, Xiu, Liu et al. 2016, Aukrust, Rosenberg et al. 2017). The nuclear localisation in particular has not been studied in great detail, although ANXA2 was reported to be present in the nucleus and to associate to the nuclear matrix (Arrigo, Darlix et al. 1983, Vishwanatha, Jindal et al. 1992, Eberhard, Karns et al. 2001, Grindheim, Hollas et al. 2016). Direct chromatin association was first shown in this work, where I demonstrated that this pool is not only nuclear but specifically chromatin-bound, emphasising the proposed role as a reader (Fig. 4.1D). When characterising the spatial expression across brain regions, I found no differences in RNA expression in hippocampal sub-regions. However, protein levels were significantly higher in the CA1 as compared to other brain regions in 3 month old mice (Fig. 4.1G-H). Immunohistochemistry did not indicate a difference in protein level between CA1 and other brain regions (Fig. 4.1F). This can be attributed to the non-denaturing conditions of the staining (in contrast to denatured in Western blot), since this has been repeatedly reported to influence ANXA2 detection in different compartments (Grindheim, Hollas et al. 2014, Grindheim, Hollas et al. 2016, Aukrust, Rosenberg et al. 2017). Since ANXA2 was low in nuclear fractions at 3 months of age, the high ANXA2 protein levels in Western blot from total CA1 lysate probably stem from high abundance in non-nuclear compartments, such as cytoplasm or extracellular space. (Fig. 4.2F). To investigate how ANXA2 changes in a model for age-associated cognitive impairment, I investigated the expression and localisation of ANXA2 in 16 month old mice. Here, ANXA2 mRNA levels and protein levels were similar between hippocampal subregions. However, CA1 showed significantly decreased protein levels when compared to 3 month old animals (Fig. 42AC), a phenomenon likely due to increased protein turnover (unpublished data, dept. Neuro- und Sinnesphysiologie, UMG, Göttingen).

To understand to what extent ANXA2 is involved in cognitive function, I produced neuronspecific AAV-mediated overexpression constructs. I injected the constructs into the hippocampus and tested the mice in cognitive tasks. In these tests I found, that mice overexpressing ANXA2 showed a spatial memory deficit. Conversely, ANXA2-deficient mice showed enhanced spatial memory in the same test. These findings suggest, that reducing levels of ANXA2 in the hippocampus is beneficial for memory performance. Thus, the observed decrease of ANXA2 in the CA1 of aged mice suggests a compensatory effect potentially aimed at mitigating age-associated memory impairments (AAMI).

Additionally, ANXA2 was enriched in the nuclei of the aged DG. Genotoxic stress is increased in ageing (Wei and Lee 2002, Strosznajder, Jesko et al. 2005, Lin and Beal 2006). However, ANXA2 was reported to translocate upon genotoxic stress and to mitigate DNA damage (Madureira, Hill et al. 2012). Thus, it is conceivable that ANXA2 translocated to the nucleus to mitigate DNA damage in ageing. This shift in turn could lead to an over-clustering of ANXA2 at 
the chromatin and reduce transcriptional plasticity, which is necessary for memory formation. This is further supported by a recent finding from Madabushi and colleagues. They demonstrated that DNA double-stranded breaks (DSBs) govern the expression of neuronal early response genes and thereby possibly memory formation (Madabhushi, Gao et al. 2015). However, DSBs are increased in the absence of ANXA2 (Appendix Fig. 1F) and inhibited in its presence (Madureira, Hill et al. 2012). This suggests that absence of ANXA2 in the CA1 of aged mice facilitates stimulus-induced gene expression to compensate for reduced transcriptomic plasticity. Conversely, genotoxic stress could promote nuclear entry in the DG of aged mice in order to reduce genotoxic stress at the chromatin, thereby reducing transcriptional plasticity.

\subsubsection{AnxA2 overexpression impairs of spatial memory}

I investigated the sub-cellular properties of ANXA2 by generating cytosolic ANXA2-GFP (ANX) and nuclear ANXA2-GFP-NLS (NLS) overexpression constructs to mimic the situation in the aged hippocampus. NLS showed a mild deficiency in the Morris water maze learning curve; however, during the probe test none of the groups displayed significant spatial representation of the platform location including controls, making it very difficult to interpret these results functionally. Thus, I applied the Barnes maze test, a more subtle and less stressful spatial memory task suitable for milder phenotypes (Harrison, Hosseini et al. 2009). In contrast to the Morris water maze, this revealed a robust spatial memory deficit in ANX, while NLS and controls memorised the task (Fig. 4.5D+E). Initially this appeared counterintuitive but could be attributed to an unforeseeable crosstalk of the recombinant NLS construct and the wild-type nuclear export signal (NES). This is particularly important since the mechanism by which ANXA2 translocates into the nucleus is not fully understood. The wild-type ANXA2 NES is located at the $\mathrm{N}$-terminus, a region reported to be crucial for protein-protein interaction, especially for $\mathrm{S} 100$ proteins which are key in ANXA2 complex formation (Eberhard, Karns et al. 2001, Nazmi, Ozorowski et al. 2012, Liu, Myrvang et al. 2015). Thus, to remove the NES the site could not be mutated to generate a nuclear ANXA2 mutant, without risking to inhibit potentially crucial interactions. Further, wild-type ANXA2 is a direct substrate of SRC kinase which phosphorylates ANXA2 at multiple sites. Of those, cytosolic phosphorylation of serine 25 appears to play a key role in initiating relocation of the protein (Liu, Rothermund et al. 2003, Liu and Vishwanatha 2007, Yan, Luo et al. 2007, Luo, Yan et al. 2008). Thus, it is assumed that ANXA2 needs to be post-translationally modified or bound to specific partners to translocate into the nucleus. To what extent these modifications are necessary for nuclear- or chromatin-dependent actions remains unknown. However, the addition of a NLS at the GFP C-terminus forced ANXA2 into the nucleus likely without undergoing necessary PTMs. After entering the nucleus ANXA2-NLS might have been exported from the nucleus immediately without taking action due to the active NES and lack of PTMs. Due to these and its initial cytosolic presence and in turn availability for post-translational modifications, only ANX could act in its physiological role. This suggests that NLS data have to be interpreted with great caution while ANX data are robust and reveal a 
significant influence of neuronal $A n x A 2$ overexpression on highly hippocampus-dependent spatial memory in vivo, without impacting other behavioural tests.

Chromatin readers are thought to act in a qualitative rather than a quantitative way. Relevant chromatin marks tend to be limited and it is the combinatorial pattern that generates differential transcriptomic outcome, opposed to the number of chromatin readers covering the available marks (Yun, Wu et al. 2011). Thus, I turned to a loss of function in vivo model to be able to further define the role of ANXA2 as a chromatin reader. Notably, AnxA2 null mice did not show alterations in locomotion or anxiety, while spatial learning was facilitated in the Morris water maze. An enhancement that was further supported by the increased use of higher cognitive strategies for learning the platform position. In short, AnxA2 KO mice used more direct search strategies that require spatial encoding and thus learned the task more efficiently. Control animals had a comparatively lower percentage of direct searching strategies and relied on slightly more random search patterns to during the task. However, this improved cognitive ability was not present during the probe test. This apparent disconnection between the learning and the memory phenotype could be due to several reasons: firstly, it might indicate differential search and learning strategies, as supported by the in-depth analysis of cognitive scores. Secondly, it could be that the Morris water maze is not a suitable task for subtle phenotypes, such as those expected in this context. As expected, the Barnes maze revealed a significantly enhanced spatial memory in AnxA2 KOs, despite a comparably smaller sample size due to limited availability of animals from this line. A main concern in any non-conditional knockout experiment is specificity of the phenotype. While developmental effects cannot be ruled out in the $\mathrm{KO}$, this issue was addressed by demonstrating an opposing behavioural effect in hippocampal overexpression of ANXA2 exclusively in neurons (Fig. 4.3D). Additionally, GSEA showed a differential impact of KO in CA1 and DG, suggesting a specific role of ANXA2 in these hippocampal areas (Fig. Appendix 1A, Fig. 4.8B). To address concerns regarding a systemic effect, a conditional or possibly CRISPR-mediated knockout could be used to recapitulate the phenotype reported.

The observed enhancement of spatial memory in ANXA2 deficiency is possibly mediated by the availability of $\mathrm{H} 4 \mathrm{~K} 12 \mathrm{ac}$ to other chromatin readers that promote memory function. Further, ANXA2 could indeed be a baseline repressor of hippocampus-dependent memory formation. Since ANXA2 acts in multifactorial complexes, ruling out involvement of functions other than the action as chromatin reader is challenging. In fact, elucidating the concrete molecular mechanism by which ANXA2 is involved in cognitive function would require systematic investigation of is partners and binding patterns in vivo. In an attempt to gain some insight into the kind of downstream processes that ANXA2 is involved in regulating, I investigated the transcriptome of $A n x A 2$ overexpression and $\mathrm{KO}$ animals using RNAseq. 


\subsubsection{ANXA2 acts from the extracellular matrix to the nucleus}

Before sequencing AnxA2 overexpression and KO mutant tissue, animals were allowed to rest for 4 days after the behavioural experiments to remove any testing bias. Differential gene expression (DE) analysis of RNAseq data showed few genes differentially expressed at a physiologically meaningful cutoff. There are mainly two possible reasons for this: masking of the neuronal ANXA2-dependent gene expression profile by non-neuronal cells or a stimulusdependent mode of action of ANXA2.

In order to test these hypotheses, I turned to an in vitro primary culture system where I could have highly pure neuronal populations, thus eliminating masking effects from other cell types. This system also allowed me to carry out different stimulation protocols under highly controlled conditions, thus enabling to study the activity-dependent mode of action of ANXA2. These experiments revealed broad but mild changes upon ANXA2 overexpression in a basal condition. A large number of genes was differentially expressed with mostly lower than 1.4 fold-changes $(\log 2 \mathrm{FC} \pm 0.5)$. To test the stimulus response in ANXA2 overexpression I used NMDA to challenge the neurons, since NMDA receptor activity was previously reported to induce ANXA2 translocation (Valapala, Maji et al. 2014). After NMDA treatment, ANXA2 overexpressing neurons showed differential gene expression compared to GFP expressing controls. While this is interesting, the results need to be treated with caution since no fold-change cutoff was applied. Differentially regulated genes with $\log 2 \mathrm{FC} \pm 0.5$ are generally considered to be more meaningful, since the potential to have a measurable influence on cellular processes is increased. Interestingly, at this cutoff several genes showed significant differential expression. In line with reports associating ANXA2 to cytoskeleton binding, this function, as well as protein and vesicle trafficking were represented, underlining the involvement of ECM deregulation (Appendix Fig. 1B)(Dityatev and Schachner 2003, Bharadwaj, Bydoun et al. 2013, Gabel, Delavoie et al. 2015).

As previously observed in behavioural experiments, I expected the loss of function model to reveal more drastic changes, due to the mode of action of chromatin readers. Accordingly, transcriptomic changes were pronounced at a $\log 2 \mathrm{FC} \pm 0.5$ cutoff in $A n x A 2$ null neuronal cultures. Importantly, functional enrichment supports the previous indications that ANXA2 acts on at least two key aspects summarised as "extracellular matrix" and "cell response". These observations support my hypotheses as to why I could observe minimal changes in vivo. I wondered whether small, individually non-significant changes in ANXA2 overexpression and AnxA2 KOs animals would nonetheless collectively represent similar functions as the ones observed in vitro. Thus, I analysed gene sets instead of differential gene expression using gene set enrichment analysis (GSEA). This confirmed an involvement of membrane-bound processes, and additionally revealed epigenetic changes and RNA processing/splicing in KO compared to WT. Interestingly, these changes were restricted to the DG, while CA1 showed only enrichment for membrane-bound processes (Appendix Fig. 1A). The involvement of membrane- 
and nuclear-bound terms again emphasised that ANXA2 has different roles dependent on its location and its interaction partners.

As a whole, transcriptomic profiling revealed several interesting functional categories, suggestive of the kinds of mechanisms by which ANXA2 might influence learning and memory. There were two main aspects: on the one hand, ANXA2's the role in sustaining the ECM and on the other to the ability of ANXA2 to translocate from the membrane to the nucleus to convey information (Reeves, Gordish-Dressman et al. 2009). While gene expression changes are significant between KO and WT neurons, it is possible that the absence of ANXA2 was in part compensated by other proteins with redundant functions. This is particularly important for proteins belonging to the same family as previously stated by Vedeler and colleagues (Vedeler, Hollas et al. 2012). In fact, AnxA1 and AnxA5, both sharing core features with ANXA2, were increased in $\mathrm{KO}$ (Appendix Fig. $1 \mathrm{C}$ ). This conceivably could have contributed to a reduced impact of ANXA2 deficiency. For example, since AnxA1 was found to be present in the cytosol (Vishwanatha, Jindal et al. 1992), it could contribute to a reduced effect of ANXA2 deficiency on cytoplasmatic function. ANXA2 was previously reported to be involved in ECM degradation and ECM remodelling (Sharma and Sharma 2007). In my experiments the impact of ANXA2 deficiency on ECM was further emphasised by GSEA, showing enrichment for the GO terms "extracellular matrix" and "extracellular matrix disassembly". Importantly, genes that contributed to these GO terms were also found to be differential expressed. Moreover, these genes have previously been linked to ECM-dependent synaptic plasticity (Star, Kwiatkowski et al. 2002, Dityatev and Schachner 2003, Bu 2009, Vasudevan, Ho et al. 2010, Dauth, Sirbulescu et al. 2011, Amenta, Creely et al. 2012, Sewal, Patzke et al. 2015, Terni and Ferrer 2015, Bach, Tzovara et al. 2017, Wiera, Nowak et al. 2017). Thus ANXA2's involvement in the extracellular matrix and its disassembly could suggest an increased ECM turnover. This is further supported by the differential expression of ECM genes relevant to synaptic plasticity. Although at this stage this is speculative, the transcriptomic analysis shed some light onto possible modes of action of ANXA2 and contributed to the explanation of the facilitated learning phenotype through an increase of synaptic plasticity based on altered ECM dynamics.

\subsubsection{ANXA2 orchestrates RNA modifications that correlate to the H4K12ac signature}

When testing ANXA2 deficient neurons in stimulus response I found substantial gene expression changes. These were associated with cellular response but particularly DNA and chromatin binding. This is in line with the previously proposed functions of ANXA2 in DNA binding, primer recognition and chromatin remodelling (Kumble, Iversen et al. 1992, Vishwanatha, Jindal et al. 1992, Boyko, Mudrak et al. 1994, Madureira, Hill et al. 2012). Further, it is conceivable that these previously reported functions are associated with the here identified role as a chromatin reader (Fig. $4.1 \mathrm{C}$ ). These findings suggest that by removing ANXA2 from the nucleus, neurons become more susceptible to stimulation and in turn transcriptionally 
active. The role of ANXA2 in the ECM and signal transduction from membrane into nucleus could arguably contribute to the increased stimulus response. I investigated the possibility that ANXA2 is involved in RNA processing directly at the chromatin, since I previously found indications of altered RNA splicing in vivo. Additionally, ANXA2 has been identified as an RNAbinding protein and is possibly also involved in RNA processing (Vedeler and Hollas 2000, Filipenko, MacLeod et al. 2004, Mickleburgh, Burtle et al. 2005, Hollas, Aukrust et al. 2006, Aukrust, Hollas et al. 2007). After showing that neurons respond to NMDA stimulation with alternative exon usage in learning-associated genes, I demonstrated that AnxA2 overexpression led to substantial exon exclusion. While little is known about bulk changes in differential exon usage, histone 3 and 4 acetylation was previously linked to exon selection through regulation of the transcriptional elongation rate (Zhou, Luo et al. 2014). A similar phenomenon has previously been described by our group, in which we found a link between deficits in H4K12ac and exon usage, with H4K12ac deficits correlating with exon exclusion, as well as changes in spatial memory (Benito, Urbanke et al. 2015). Further, acetylation of $\mathrm{H} 4$ was linked to alternative exon usage by modulation of the elongation rate (Izquierdo 2008, Hnilicova, Hozeifi et al. 2011). Thus, an increase of histone acetylation facilitated RNA polymerase II (RNAPol-II) and exon exclusion in 3 different instances (Schor and Kornblihtt 2009, Hnilicova, Hozeifi et al. 2011, Zhou, Hinman et al. 2011). In fact, the exon exclusion observed in AnxA2 over-representation closely resembles our previous finding when acetylation of H4K12 was increased. This could suggest that the effect of hyper-acetylation at histone 4 acts by a similar mechanism on splicing as over-representation of the chromatin reader at the same mark. Importantly, this observation was sustained by the opposing effect, significant exon inclusion, in the ANXA2 deficient condition. This is in line with the effect observed in hypo-acetylated H4K12 and correlates with the behavioural observations. Investigation of the processes affected by alternative exon usage revealed similar GO terms associated with cytoskeletal structure and neuronal extension in both overexpression and $\mathrm{KO}$ neurons, while the latter showed more complex associations to cellular projection and synaptic transmission. Importantly, differential exon usage targeted particularly the microtubuli network including the microtubule-associated protein TAU, which could be a possibility to ameliorate pathophysiological changes associated with AD or other Tauopathies' progression (Hempen and Brion 1996). Further, the enriched GO categories in differential exon usage of both $A n x A 2 \mathrm{KO}$ and overexpression, suggest differential priming of neurons. Thus, one could speculate that the altered cytoskeleton structure and specifically changes in molecular motors would contribute to ready-to-react state in which signal transmission would be facilitated. This notion is further supported by the specific signature after NMDA stimulation. Here, enriched GO terms were closely linked to vesicle release and learning and memory. Clusters were generally more extensive in KO neurons, which is in line with overall altered gene response in KO (Fig. 4.10G). Exon skipping was increased in the overexpression experiment, suggesting increased elongation rate and interference caused by over-clustering (cf. "kinetic coupling", chapter "1.4.4. On chromatin assisted alternative splicing"). Importantly, 
also ANXA2 deficient neurons showed significant exon exclusion. In contrast to overexpression and wild-type, exon exclusion in $\mathrm{KO}$ occurred vastly more frequently in proximity to TSS (Fig. 4.14C). Exon exclusion occurs in both $\mathrm{KO}$ and overexpression, which could indicate a similar fundamental mechanism. However, KO show a more specific outcome with alternative exon usage accumulating at the TSS. It is therefore conceivable that $A n x A 2 \mathrm{KO}$ leads to a very specific change within the gene topology, while overexpression acts less precisely on the entire gene body. While this is speculative, the lack of ANXA2 could lead to the initiation of transcription at an alternative site downstream of the original mark. During Polll initiation short $\sim 50$ bp sequences of the 5' are transcribed before elongation (Kwak, Fuda et al. 2013). Thus the observed decrease of exons in the 5' could point towards facilitated or alternative PollI initiation, leading to base skipping and in turn exon exclusion in the first $10 \%$ downstream of TSS. In KO neurons, the exon skipping at the TSS upon stimulation could indicate facilitated initiation of the transcriptional machinery, correlating with H4K12ac, as indicated by ChIPseq. Thus ANXA2 deficiency could cause faulty transcription of initial sequences due to improper assembly of the transcriptional machinery (cf., "chromatin splicing adaptor system", chapter "1.4.4. On chromatin assisted alternative splicing").

Genes affected by this event were mostly associated with energy pathways and particularly NAD metabolism, an effect not observed for any other condition. Based on the target genes of 5 ' exon skipping and our initial screen one could hypothesise that in the KO condition H4K12ac is read by an alternative chromatin reader. Although this idea clearly requires further investigation, it is tempting to speculate that the alterations in NAD metabolism could could be due to a replacement of ANXA2 by PARP1 (ADPRT1). This reader was also identified in our initial screen and is strongly tied to NAD metabolism (Luo and Kraus 2012).

In summary, we identified several novel readers of acetylated H4K12, including ANXA2. I showed that ANXA2 protein is present in the nuclei of DG cells, while it decreases in the CA1 in the ageing brain. I further demonstrated that ANXA2 and has a significant impact on spatial memory. The data suggest that ANXA2 accumulation in the nucleus of the DG in aged animals could constitute a detrimental phenotype in ageing and that the reduction in CA1 compared to young mice would be a compensatory mechanism to counteract age-associated memory impairment. This effect is most likely conveyed by an interplay of the complex roles ANXA2 takes in different cellular compartments. A multifactorial protein like ANXA2 cannot be reduced to a single outcome as this would be disadvantageous to the interpretation of the data. However, the data suggest that the mechanism can be separated into non-nuclear (ECM, signal transmission) and nuclear (chromatin reader, differential exon usage) effects. Firstly, ANXA2 could contribute to ECM stability and reduce turnover, which is why a reduction of ANXA2 levels could lead to improved spatial memory, possibly through enhanced synaptic plasticity. Conversely in its nuclear role ANXA2 influences alternative exon usage. Importantly, the outcome of the ANXA2 orchestrated alternative exon usage events is analogous compared to the manipulation of H4K12ac itself (Benito, Urbanke et al. 2015). In fact, to the best of my 
knowledge, this is the first report of ANXA2 as a possible key player being instrumental in translating histone PTMs into alternative splicing. Given the data presented here and the previously reported beneficial effect of reinstating H4K12 acetylation on cognition, I propose to further investigate on the beneficial influence of ANXA2 deficiency in age-associated memory impairment and cognitive decline.

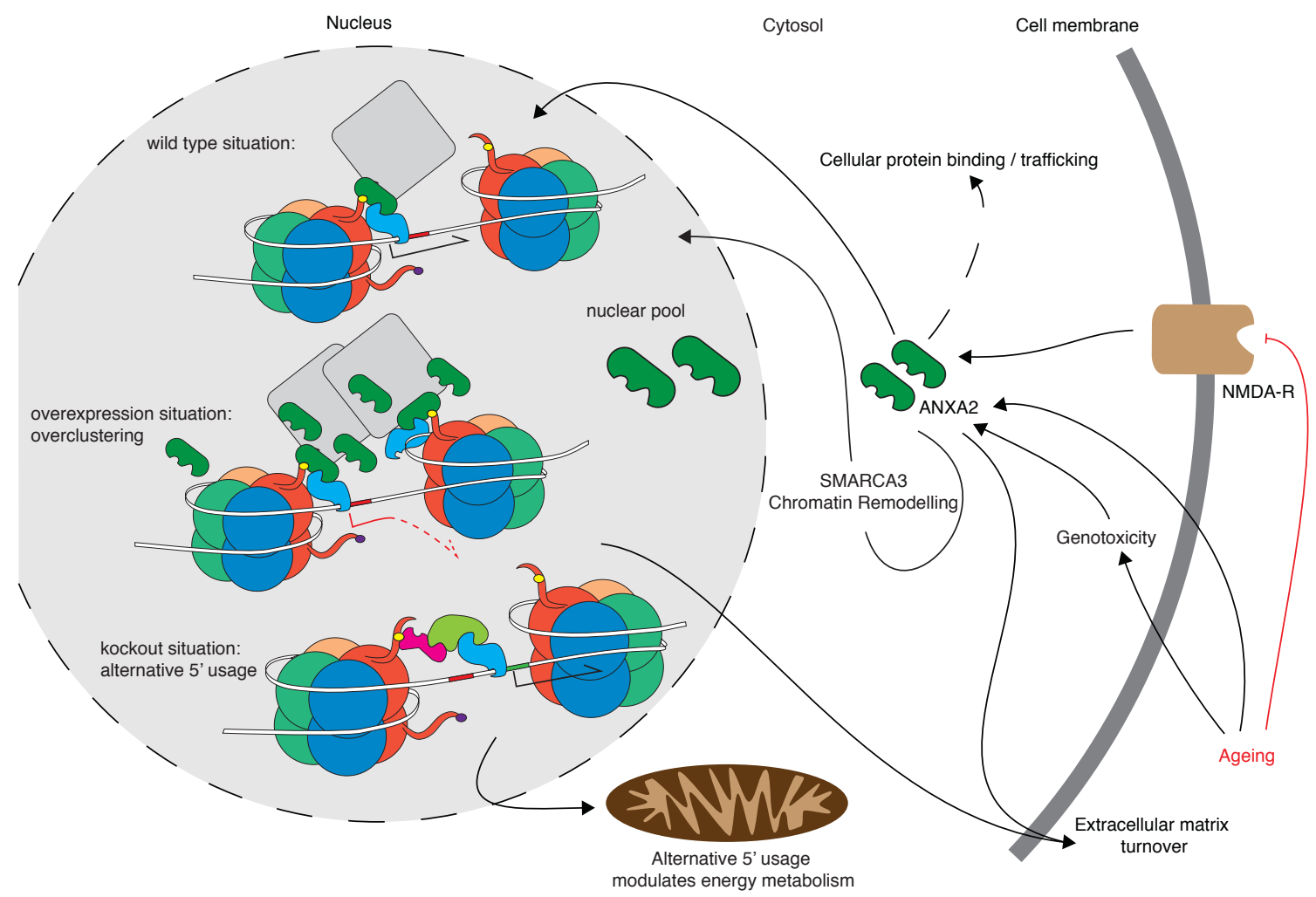

Figure 4.22: A model of the multifactorial chromatin reader ANXA2 in neurons including relevant literature.

ANXA2 is responsive towards ageing (possibly through genotoxicity) and NMDA stimulation. ANXA2 is involved in cellular protein binding and trafficking of e.g. RNAs or vesicular bodies. Further, ANXA2 in associated with the extracellular matrix and possibly its turnover. ANXA2 was reported to be part of the chromatin remodelling complex SMARCA3 and to translocate into the nucleus. In this work we demonstrated that ANXA2 binds to acetylated H4K12 where it likely serves as a scaffold to initiate transcription or in parallel RNA processing. When ANXA2 is overexpressed, severe exon skipping along the entire gene body is observed, which indicates malfunction of the elongation process. This could possibly be mediated by steric inhibition due to increased number of transcription initiation sites and thus excessive kinetic coupling during elongation. When ANXA2 is absent exon skipping is most significant in the 5' of energy metabolism associated genes. This can be explained by either another chromatin reader taking over ANXA2s role, however, initiating at a downstream site, or a malfunctioning RNA PollI initiation.

\subsubsection{A side note on ANXA2 life-long deficiency in learning and memory}

In an alternative line of experiments I found ANXA2 to inhibit DNA double strand breaks in neurons (Appendix Fig. 1F). At the same time it was demonstrated that DSBs are relevant to the induction of immediate early genes and possibly in turn memory formation (Madabhushi, Gao et al. 2015). Connecting these two observations, I hypothesised that the increase of DSBs in 
AnxA2 KOs would contribute to an increased in immediate transcriptional response. I was able to verify the increased susceptibility to stimulus driven gene expression in differential expression analysis of RNAseq data (Fig. 4.10G). However, it is not clear whether this change is indeed causally linked to increased DSBs.

In the ageing brain the repeated induction of DSBs in the hippocampus could in the long-term, be detrimental due to increased probability of mutations and thus impaired transcription. Thus, I hypothesised that aged animals would show decreased memory. I tested ANXA2 deficient animals in the Barnes maze test for spatial memory and found that mice at 13 months of age showed a memory impairment compared to wild-type control (Appendix Fig. 1G-K). At the time of writing this phenomenon needs further investigation, especially in respect to the influence on the reported function of ANXA2 in splicing, but underscores the relevance of ANXA2 in learning, memory and ageing.

\subsection{HDAC6 modulation and Anle138b: two convergent strategies as novel therapeutic avenues to treat $A D$}

Alzheimer's Disease is a detrimental neurodegenerative disorder affecting 47 million people world wide. This puts a great burden on patients affected and their family, but also a socioeconomical challenge our society as whole. While the disease mechanisms are not fully understood, two molecular aggregopathies have been identified, namely neurofibrillary tangles of TAU (NFTs) and AB plaques. Both pathologies have been of major interest in research of AD. However, to date there is no efficient treatment available to ameliorate or even cure the disease. Clinical trails targeting either NFTs or $A B$ plaques have failed Phase II clinical trials of antiamyloid $\beta$ antibodies (Panza, Frisardi et al. 2012, Gold 2017). An alternative approach to reduce the socio-economic burden and to improve late-life quality in the short-term is to develop symptomatic treatments. A promising approach is to circumvent the molecular pathologies and instead reinstate cellular functionality indirectly, e.g. by reinstating transcription or cellular integrity. The development and progression of sporadic $A D$ is closely associated with environmental risk factors. This puts the epigenetic machinery into focus, since it translates environmental input into cellular outcomes. A promising target of the epigenetic machinery is HDAC6, which deacetylates TAU and microtubuli. Both of these processes are detrimental factor in AD progression (Cook, Stankowski et al. 2014). Importantly, removal and inhibition of HDAC6 has already been shown to be beneficial in AD-like amyloidosis. Thus I tested the effect of HDAC6 deficiency in a mouse model for AD-like tauopathy and found a reinstatement of anxious behaviour.

In a second set of experiments we tested the novel compound anle138b in AD-like amyloidosis. We report that anle138b reinstates to some extent synaptic plasticity and spatial memory. This effect is possibly mediated through reinstatement of membrane integrity by inhibiting the functionality of $A B$-pores. This finding is of particular encouraging since anle138b was reported 
to act beneficial in other aggregopathies including AD-like tauopathy (Wagner, Krauss et al. 2015).

\subsection{HDAC6 deficiency rescues anxiety in the THY-Tau22 mouse model for $A D$}

In patients suffering from AD or other Tauopathies significantly increased HDAC6 levels are observed. HDAC6 reduces tubulin and TAU acetylation levels, which in turn is associated with disturbance of the microtubule network and correlates negatively with disease progression (Hempen and Brion 1996, Ding, Dolan et al. 2008, Kim, Choi et al. 2012).

Thus, I wondered whether decreasing HDAC6 in a Tauopathy model could be beneficial for disease pathophysiology. This strategy has proven useful in the past, where reducing HDAC6 in an APP mouse model led to improved cognitive performance (Govindarajan, Rao et al. 2013). By crossing TAU HDAC6-/- to THY-Tau22 mice, I investigated the effect of removing HDAC6 on TAU pathology. To test previously described phenotypes of the THY-Tau22 model in anxiety, locomotion, and hippocampus-dependent memory, I conducted a broad battery of behavioural tasks. In line with a previous report (Schindowski, Bretteville et al. 2006) I demonstrated a reduction of anxious behaviour in TAU transgenic animals in late-stage pathology. Importantly, this deficit was completely rescued by HDAC6 deficiency. In contrast to a previous report (Schindowski, Bretteville et al. 2006), I did not observe reduced anxiety in TAU transgenic animals at early-stage. A possible explanation might be increased genetic variance after crossing THY-Tau22 and Hdac6-- despite the identical C57B6j background, although other factors cannot be excluded. I found decreased anxiety in TAU transgenic animals in the Open field, a test that has previously not been reported for this mouse line. In contrast to the Elevated plus maze (EPM), anxiety was decreased in the TAU animals at early-stage (6 month old) as well as late-stage (13 month old), and HDAC6 deficiency did not ameliorate the phenotype. Notably, I did not observe any significant motility impairment in TAU transgenics, which is in line with previous reports (Schindowski, Bretteville et al. 2006, Van der Jeugd, Blum et al. 2013). None of the early-stage cohorts showed a significant improvement in spatial memory. While controls displayed the shortest escape latency, it was still unusually high and might explain the lack of difference between mutants and controls. In contrast, at late-stage pathology TAU transgenic animals showed significantly increased escape latencies, as previously reported (Schindowski, Bretteville et al. 2006). HDAC6 removal did not significantly ameliorate this defect. Interestingly, I detected a previously not reported increase of contextual fear memory in Hdac6 $\mathrm{KO}$ compared to controls regardless of TAU transgene expression in both the early-stage and late-stage cohorts. This observation could be due to the change in genetic background of

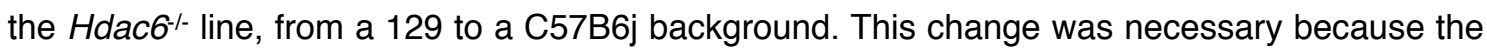
former has previously been reported to be less suitable for cognitive testing.

Taken together, these findings show in vivo evidence for reinstatement of behavioural abnormalities due to genetic HDAC6 deficiency in any model of TAU pathology, more 
specifically anxiety behaviour. In line with this finding, a previous study reported that pharmacological HDAC6 inhibition by tubastatin in the rTg4510 mouse model for TAU deposition restored memory function and reversed a hyperactivity phenotype (Selenica, Benner et al. 2014). In contrast to other models, including rTg4510, the THY-Tau22 mouse model does not develop motor deficits (Schindowski, Bretteville et al. 2006), a crucial prerequisite for reliable behavioural testing.

Post-translational modifications (PTMs ), especially phosphorylation, of TAU were in the past well investigated. However, little is known about the effect of acetylation. HDAC6 inhibition was shown to directly increase Tau, tubulin, and microtubule acetylation (Zhang, Li et al. 2003, Noack, Leyk et al. 2014). Elevated HDAC6 activity was reported to increase phosphorylation of TAU within Tau's microtubule-binding domain by an unknown mechanism (Cook, Stankowski et al. 2014). It is assumed that both phosphorylation and acetylation of TAU within the microtubule binding repeat region decreases affinity towards microtubuli, which would in turn facilitate axonal transport by molecular motors such as dynein and kinesin-1 (Drechsel, Hyman et al. 1992, Biernat, Gustke et al. 1993, Bramblett, Goedert et al. 1993). Additionally, acetylation of TAU inhibits its polymerisation and could potentially lead to reduced intracellular deposits (Cook, Carlomagno et al. 2014) and inhibition of HDAC6 suppressed neuritic TAU bead formation in neurons and mice, a process correlating with plasticity impairment (Tseng, Xie et al. 2017). Thus the reinstated anxious behaviour through removal of HDAC6 is possibly due to an increase of microtubule stability and enhanced clearance of pathogenic TAU species. The latter is likely mediated trough high acetylation levels which inhibits TAU phosphorylation and aggregation.

Further, our group previously reported that loss of HDAC6 ameliorates hippocampus-dependent memory deficits and renders neurons resistant to amyloid- $\beta$-mediated impairment of mitochondrial trafficking, likely trough enhanced tubulin acetylation (Govindarajan, Rao et al. 2013). This is in line with findings showing that tubulin acetylation enhanced the recruitment of kinesin-1 and dynein (Dompierre, Godin et al. 2007) and is a possible mechanism by which HDAC6 deficiency could contribute to the restoration of anxiety behaviour. While the precise mechanism remains unknown, the data presented reveal a beneficial effect of HDAC6 deficiency in a TAU pathology model for $A D$, which is also relevant to other Tauopathies and encourages further investigation on the underlying mechanism. Importantly, our group demonstrated that HDAC6 deficiency contributes to an amelioration of behavioural phenotypes in APP and TAU models, the two main molecular hallmarks of AD. This would be the first report of an entity that interacts with both molecules and provides a cognitive benefit. A specific inhibitor to HDAC6 would therefore be a highly promising candidate for clinical trials in AD. Such an inhibitor would be the first drug to ameliorate both molecular pathologies of $A D$, a finding that is only pre-empted by the herein presented results on the compound anle138b. 


\subsubsection{Anle138b rescues LTP and ameliorates spatial memory deficits in APP/ $P S 1$ mouse model for $A D$}

We used the APP/PS1 mouse model for AD to investigate the potential of the diphenylpyrazole anle138b as a therapeutic agent in AD. The APP/PS1 mouse model for AD is well-established and recapitulates neuronal dysfunction. In these mice memory decline can be detected after four months and plaques form after 6 months of age (Kummer, Hammerschmidt et al. 2014).

Anle138b was previously demonstrated to have beneficial effects in models for aggregopathies such as Parkinson's and Alzheimer's disease (Levin, Schmidt et al. 2014, Deeg, Reiner et al. 2015, Wagner, Krauss et al. 2015, Fellner, Kuzdas-Wood et al. 2016). The compound is a hydrophilic and intended to modulate causative factors of AD, such as amyloid aggregation. Anle138b was shown to be administrable via food pellets and able to enrich in the brain, while remaining non-toxic up to a dose of $2 \mathrm{~g} / \mathrm{kg}$ (Wagner, Krauss et al. 2015). We found that oral administration of anle138b restored LTP in the pre- and post-plaque cohort and that it reinstated spatial memory fully in the pre-plaque and partially in the post-plaque cohort. Notably, we did not observe any effect of anle138b treatment on wild-type animals, which is reassuring in terms of translating the use of this compound into the clinic. Transcriptome analysis of the pre-plaque group showed full reinstatement of genes linked to metabolic function and neuronal plasticity, which were down-regulated in the placebo group. Interestingly, few neuroinflammatory terms were enriched even in the placebo group. In contrast, changes in the post-plaque placebo group were dominated by increased inflammation and anle138b treatment did not impact transcriptomic deregulation. Yet, a significant therapeutic effect was observed in the post-plaque group, reinstating synaptic function despite detrimental inflammatory processes. In light of clinical trials that target amyloid deposition, a main argument for the failure of such trails is that clearance of plaques is ineffective at later stages of the disease. Anle138b treatment, seems to circumvent this mechanism, nonetheless targets pathological processes, in turn allowing synaptic functionality to recover despite persisting inflammatory processes.

One previously proposed mechanism that is compatible with our results is the pore formation hypothesis. It states that neurotoxic compounds, such as $A \beta$ oligomers, form permeable pores that impair neuronal function and lead to cell death (Arispe, Rojas et al. 1993). However, experimental evidence existed only to a limited degree, since a combination of different in vivo and in vitro techniques was required to test this notion.

A main indication for the proposed mechanism stems from our observation using artificial bilayer membranes, so called black lipid membranes (BLM), which show little conductance when intact. In our experiment we observed a stepwise growth of membrane conductance after $A \beta$ application, which suggests a combined action of several individual pores in the BLM. Earlier experiments have shown that $A \beta$ mutated at F19P shows barrel structure but no ionic conductance (Capone, Jang et al. 2012, Connelly, Jang et al. 2012). The F19P mutation also changes in $\beta$-sheet confirmation, which collapsed the pore (Connelly, Jang et al. 2012). Our data are in line with these findings and suggest that anle138b induces a conformational change 
in the $A \beta$ pores, which reduces or eliminates ionic conductance. Our in vivo evidence, demonstrating reduced membrane integrity in neurons after $A \beta 1-40$ application, further supports this idea. Importantly, this effect was abolished when neurons were supplemented with anle138b before or after $A \beta 1-40$ treatment, mimicking the pre-and post-plaque cohort respectively. This potential "patching" of $A \beta$ pores and in turn protective effect on membrane integrity is in line with our findings in the post-plaque group, where the compound reinstated LTP without affecting gene expression.

It is very likely that anle138b acts through additional mechanisms, as it has also been shown to be beneficial in models of other aggregopathies such as Parkinson's disease (Levin, Schmidt et al. 2014), Creutzfeldt-Jakob (Wagner, Ryazanov et al. 2013), and TAU pathology (Wagner, Krauss et al. 2015). The interaction with TAU is particularly interesting, because this would be the first report of a compound being beneficial for amyloidosis and aggregation of tau, thus targeting two molecular hallmarks of $A D$.

\subsection{Illuminating neuronal network dynamics}

Our brain is composed of around 85 billion neurons and approximately as many glia cells (Azevedo, Carvalho et al. 2009). For future treatment and cure of neurodegenerative diseases it is essential to understand the precise interplay between neurons in the complex network of the brain. It is not understood how neuronal activity propagation influences the surrounding network and how feedback from adjacent cells changes the transcriptome of a formerly active cell. To decipher these network dynamics on a single cell level few model systems are available and lack either accessibility, spatial precision, temporal resolution or clinical relevance. Thus I built a now model system addressing these current obstacles to study neuronal network behaviour on a single cell level.

The system is comprised of a biological component and second a hardware component. The former allows to introduce a genetic modification into a tightly controlled sub-population of cells in culture without depending on different promotors. The latter is a optogenetic stimulation unit tailored for the needs of cell cultures application for molecular biology.

In the described experiments, I composed accurately titrated networks from neurons with two mutually exclusive ectopically expressed genes. First "pacemakers", a subpopulation of light activatable neurons expressing channelrhodopsin which is primarily stimulated cells. Second "reporters", which were secondarily stimulated by pacemakers and expressed eYFP upon activation. After the experiment each cell was extracted from the network and subjected to high throughput single cell sequencing depending on their ectopically expressed gene.

On the biological side, I developed a protocol that results in highly targeted gene delivery. To transmit genetic information I relied on adeno-associated viruses (AAVs) as vectors because of several reasons. AAVs are safe to handle and easy and economical to produce (McClure, Cole 
et al. 2011). They are not associated with any known human disease but generate long-term transgene expression in non-dividing cells such as neurons and display relatively low immunogenicity (Rapti, Louis-Jeune et al. 2012). Further, AAV serotypes (AAV1 to AAV13) differ in structures of their capsids and offer in turn differential infectious properties (AgbandjeMcKenna and Kleinschmidt 2011). For many AAV serotypes it was reported that binding to cell surface glycans is crucial for infection (Summerford and Samulski 1998, Handa, Muramatsu et al. 2000, Kaludov, Brown et al. 2001). In contrast, AAV2 was shown to bind heparan sulfate proteoglycans and can be competitively inhibited by heparin (Summerford and Samulski 1998, Schmidt, Voutetakis et al. 2008). All of these features were important in the choice of AAV for construct delivery. However, the outstanding property to be inhibited by heparin made me select of AAV serotype 2, as this allowed inhibition of viral spread during long-term incubation. Thus, to generate a system for specific construct introduction I relied on AAV serotype 2, and 1/2 hybrids and inhibited propagation during long term incubation ( $>10$ days) by supplementing heparin to the medium to sponge viral particles released from dissociating cells. Additionally, the relatively low infection rate of AAV2's can efficiently be counteracted with high titers and thus enables short infection incubation times as it was necessary for pre-seeding construct delivery (Kern, Schmidt et al. 2003).

The protocol was then used by Lukas Faiß, under my supervision, to deliver ChR2 into $10 \%$ of a primary neuronal culture (PNC) and found FOS increase in cells stimulated by ChR2+ but not in pacemakers themselves, a finding that is contrary to current literature where fully transfected cultures showed increase in FOS levels after simulation (Schoenenberger, Gerosa et al. 2009). This led us to hypothesise that not only the stimulus quality but also the amount and intensity of feedback to the pacemaker is relevant to the stimulus response. To investigate this phenomenon in more detail I devised an experiment in which $0 \%, 1 \%, 10 \%, 30 \%$, or $50 \%$ pacemakers were introduced into a reporter culture. The cells were then stimulated with LuCIFR-prototypeB according to published protocols (Schoenenberger, Gerosa et al. 2009), dissociated, FACS sorted and subjected to single cell sequencing using MARS. Unfortunately, at the time of writing this thesis the outcome is pending. However, I am positive it will give more insight into how active neurons influence the transcriptional level of neighbouring cells and what role the amount of active cells plays in neuronal network activation.

The system established now allows to introduce almost any genetic mutation into dissociated primary neuronal cultures, neuronal/glia hybrid cultures, 3D cultures or any other system where an infection before seeding can be applied. Thanks to the continuous improvement of genetic tools to introduce and remove target genes, this approach can be adapted for any number of cell culture experiments future investigators will have in mind.

Complementing the construct delivery system I engineered the optogenetic stimulation device "LuCIFR" which is currently in licensing negotiations with partners in industry, unfortunately this prohibits a description of the current technology. However, LuCIFR is designed as the ideal complement to the previously described genetic construct delivery protocol to introduce 
optogenetic tools into cell culture and circumvents disadvantages of currently available devices. To our knowledge LuCIFR is the first stimulation unit designed for high throughput and entry level molecular-biology cell culture experiments. For exploratory optogenetic work in traditionally molecular biology laboratories the acquisition of commercially available stimulation solutions is too expensive, which in turn forces them to build self-made devices, often with the outcome of a light stimulus of unknown parameters and limited control of the same. Although, publications describing instructions for good optogenetic devices exist (Grossman, Poher et al. 2010, Gerhardt, Olson et al. 2016), availability of workshops to build such devices is restricted to most labs. However, since precision and standardisation are key prerequisites to reproduce an experimental outcome, LuCIFR was designed to protocol any event, to be rugged, waterproof, highly precise, and reproducible, while remaining affordable to anyone and thus outperforming self-made and commercial devices alike. 


\section{Summary}

Alzheimer's disease $(A D)$ is the most common dementia-associated neurodegenerative disorder. It has detrimental social and economic impact not only on patients, but also their family and society as a whole. Current estimations predict a total of 131 million affected with the disease in 2050 (World Alzheimer Report, 2016), increasing socio-economic pressure. To date there is no cure and no efficient treatment against $A D$ available. Appearance and progression of $A D$ depend on the interplay between genetic and environmental risk factors, which is intimately linked with the epigenetic machinery, since it is exactly at the interface between the environment and the genome. With the aim of exploring novel strategies to ameliorate or prevent ageassociated dementia, I used 3 complementary approaches that led to the identification of suitable drug- and genetic-based targets.

First, to identify novel drug targets I performed a screening for proteins specifically binding the memory-associated histone mark H4K12ac. I identified ANXA2 as a significant chromatin reader. I could show that ANXA2 not only changes sub-cellular localisation during ageing, but is causally involved in cognitive function. My findings suggest that ANXA2 is a baseline inhibitor of spatial memory function. Since ANXA2 is naturally multifactorial, it is challenging to pinpoint a precise mechanism. However, my data indicates that ANXA2 influences two key aspects relevant to learning and memory: on the one hand it contributes to extracellular matrix dynamics, which has previously been reported to be a regulator of synaptic plasticity. On the other hand, I found evidence that supports a role of ANXA2 in modulating energy metabolism through alternative 5' usage. Although I did not manipulate ANXA2 in an AD model, my observations suggest that inhibiting it could be a suitable strategy to ameliorate memory impairment in AD.

Second, to test whether previously identified targets could constitute novel treatment strategies against AD, I explored the benefits of two pharmacological compounds (JS28 and anle138b) and a genetic model of HDAC6 deletion (Tau/HDAC6). My findings on HDAC6 depletion show a reversal of the common anxiety phenotype in a mouse model for AD-like tauopathy. This suggests that specific inhibition of HDAC6 could be a suitable treatment strategy specifically aimed at normalising anxiety in $A D$. This is complemented with other findings reporting a benefit of HDAC6 depletion in AD-like amyloidosis (Govindarajan, Rao et al. 2013). One of the pathological $A D$ mechanisms is the pore formation hypothesis. It states that $A B$ oligomers pierce through neuronal membranes forming ion-channels and thereby shorting signal transduction and causing cell death. We assessed the efficacy of anle138b as a pore formation inhibitor upon $A B$ exposure and report highly beneficial effects of the molecule in a recent issue of "EMBO molecular medicine". Anle138b reinstated LTP and spatial memory even at late stages of amyloid deposition in the APP/PS1 mouse model for AD-like amyloidosis. We present evidence that anle138b could act through induction of conformational changes to the $A B$ pore structure and that membrane integrity is reinstated after anle138b treatment both in vitro and in vivo. This 
supports previous findings that suggested a beneficial function of anle138b in other aggregopathies, including AD-like tauopathy.

Third, I developed a new technical strategy aimed at studying network dynamics. In order to understand complex diseases, such as $A D$, it is essential to study neurons in the network and go beyond cellular events to systems-level events. I designed and optimised a novel AAV-based vector system to introduce genetic constructs into neuronal subpopulations. I demonstrated the effectiveness of this approach in a proof-of-principle experiment, introducing channelrhodopsin to investigate the influence of low-density activity on neuronal networks. Importantly, this system is highly customisable and could be adapted for a broad range of applications. To complement the channelrhodopsin-driven neuronal network activity, I designed and engineered a new optogenetic stimulator for cell culture (LuCIFR). By combining vector-based subpopulation targeting with FACS sorting, LuCIFR-based stimulation and single-cell sequencing, I established a new technical approach to study network dynamics in subpopulations and individual cells.

In this thesis I explored several molecular pathways involved in cognitive function with an emphasis on therapeutic applications. I identified ANXA2 as a chromatin reader and inhibitor of spatial memory function, contributed to the characterisation of 2 highly novel $A D$ treatment strategies, and developed a new system to decipher neuronal network dynamics. While each of these topics comprises its own field for future investigations, I am positive they will impact neurodegenerative and neurocognitive research and hopefully contribute to future treatments of cognitive impairments and AD. 
$\frac{X}{8}$
$\frac{8}{8}$
$\frac{8}{7}$ 


\section{Appendix}

Appendix DVD

\section{Appendix Figure 1}

CA1 in vivo GSEA showed lower overall enrichment and mostly Membrane-bound processes (padj $<0.05$, FDR<0.25) (A). Differential gene expression of basal ANXA2 overexpressing neurons with increased cutoff (padj<0.05, log2FC \pm 0.3 ) showed enrichment in GO ORA analysis of molecular function exclusively fibronectin binding (B). AnxA1 and AnxA5 were increased in RNAseq differential expression analysis in AnxA2 KO neurons (C). KEGG pathway analysis of differentially used exons in WT NMDA treated neurons compared to basal showed oxidative phosphorylation enriched (D). KEGG pathway analysis of differentially used exons in ANX NMDA treated compared to GFP NMDA treated neurons showed no enrichment (E). Wester blot analysis of neurons treated with $200 \mu \mathrm{M} \mathrm{H} 2 \mathrm{O} 2$ showed increased DNA double strand breaks as indicated by $\gamma \mathrm{H} 2 \mathrm{~A} . \mathrm{x}$ signal in the absence of ANXA2 (F). 13 month old AnxA2 null and WT mice travelled similar distance during the first probe (PT1) test in the Barnes maze test for spatial memory. Importantly, KO mice spent significantly less time in the target area during PT1 (G, left). PT2 after 4 additional training sessions in the Barnes maze showed both cohorts travel similar distances. Importantly also KO were able to learn the task and spent significantly more time in the target area (G, right). 
A Gene Set Enrichment in KO CA1

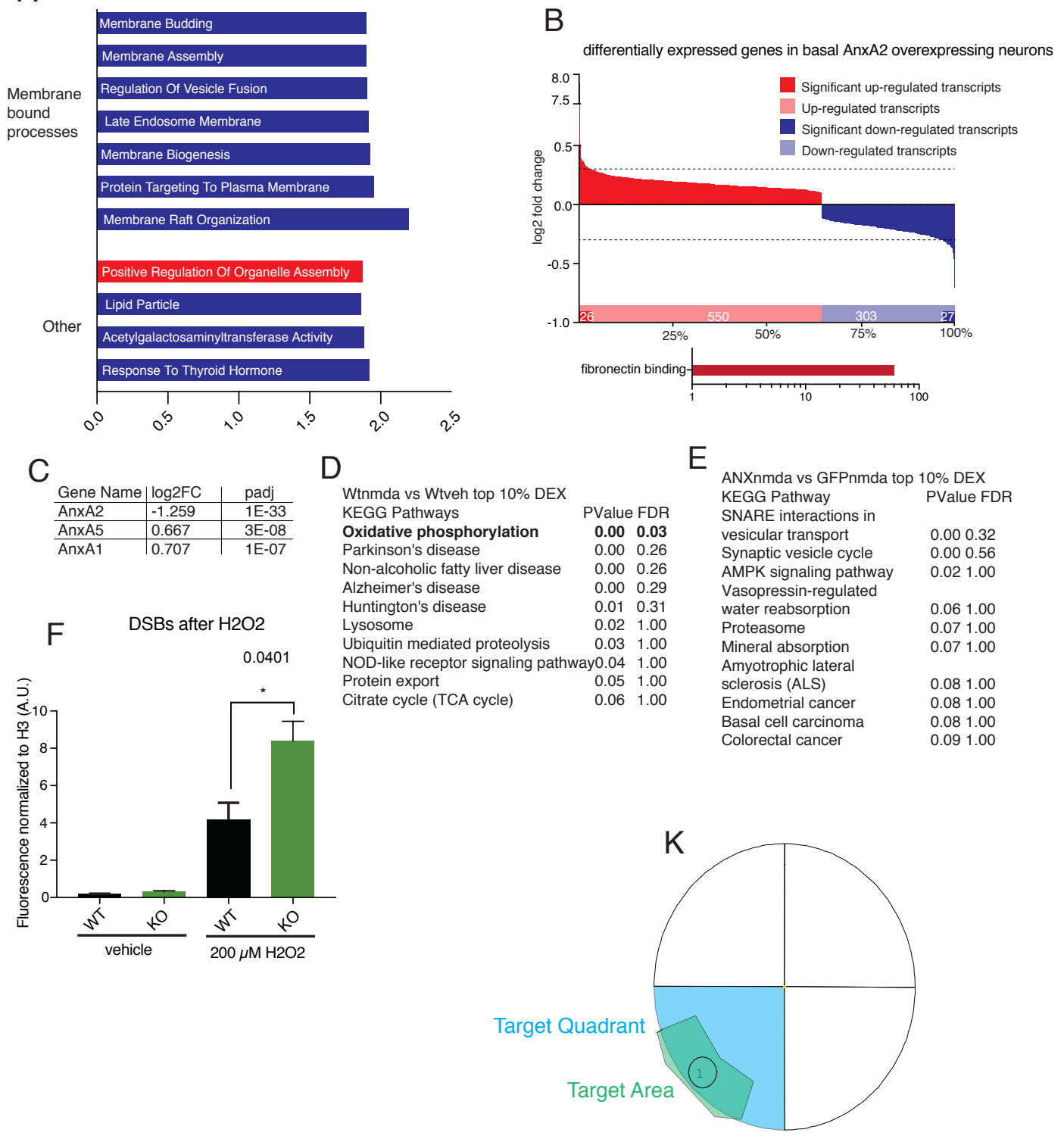

G

PT1 distance travelled aged BM PT2

aged BM PT2

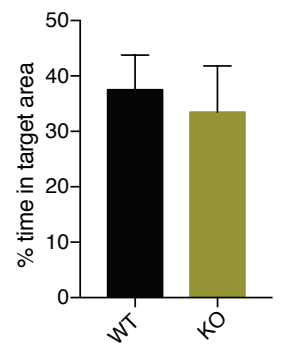

PT2 distance travelled

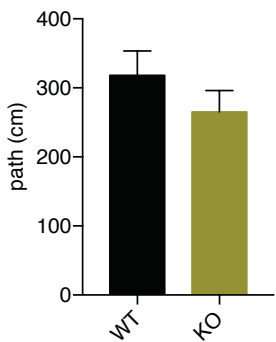


Appendix Table 1: Top 10\% TSS proximity exons differentially expressed in NMDA treated KO vs. WT

\begin{tabular}{|c|c|}
\hline genesymbol & genename \\
\hline Epdr1 & ependymin related protein 1 (zebrafish) \\
\hline Prkab2 & protein kinase, AMP-activated, beta 2 non-catalytic subunit \\
\hline Napg & $\mathrm{N}$-ethylmaleimide sensitive fusion protein attachment protein gamma \\
\hline Napa & $\mathrm{N}$-ethylmaleimide sensitive fusion protein attachment protein alpha \\
\hline R3hdm4 & $\mathrm{R} 3 \mathrm{H}$ domain containing 4 \\
\hline Rhoa & ras homolog family member $A$ \\
\hline Atp5f1 & ATP synthase, $\mathrm{H}+$ transporting, mitochondrial F0 complex, subunit B1 \\
\hline Cd81 & CD81 antigen \\
\hline Crym & crystallin, mu \\
\hline Cyp51 & cytochrome P450, family 51 \\
\hline Eno2 & enolase 2 , gamma neuronal \\
\hline Gpi1 & glucose phosphate isomerase 1 \\
\hline Hint1 & histidine triad nucleotide binding protein 1 \\
\hline Igfbp3 & insulin-like growth factor binding protein 3 \\
\hline Mdh2 & malate dehydrogenase 2, NAD (mitochondrial) \\
\hline Mapt & microtubule-associated protein tau \\
\hline Nnat & neuronatin \\
\hline Mrpl49 & mitochondrial ribosomal protein L49 \\
\hline Pea15a & phosphoprotein enriched in astrocytes 15A \\
\hline Psen1 & presenilin 1 \\
\hline Eif4a3 & eukaryotic translation initiation factor $4 \mathrm{~A} 3$ \\
\hline Rab1a & RAB1A, member RAS oncogene family \\
\hline Rad23b & RAD23 homolog $B$, nucleotide excision repair protein \\
\hline Mcfd2 & multiple coagulation factor deficiency 2 \\
\hline Ran & RAN, member RAS oncogene family \\
\hline Sstr2 & somatostatin receptor 2 \\
\hline Spr & sepiapterin reductase \\
\hline Srp14 & signal recognition particle 14 \\
\hline Timp3 & tissue inhibitor of metalloproteinase 3 \\
\hline Ube2b & ubiquitin-conjugating enzyme E2B \\
\hline Vdac1 & voltage-dependent anion channel 1 \\
\hline Ywhag & tyrosine 3-monooxygenase/tryptophan 5-monooxygenase activation protein, gamma polypeptide \\
\hline Atp5g3 & ATP synthase, $\mathrm{H}+$ transporting, mitochondrial F0 complex, subunit C3 (subunit 9) \\
\hline Dlat & dihydrolipoamide S-acetyltransferase (E2 component of pyruvate dehydrogenase complex) \\
\hline Map2k1 & mitogen-activated protein kinase kinase 1 \\
\hline Ankrd13b & ankyrin repeat domain $13 b$ \\
\hline Rpl8 & ribosomal protein L8 \\
\hline Srr & serine racemase \\
\hline Tnpo3 & transportin 3 \\
\hline Atraid & all-trans retinoic acid induced differentiation factor \\
\hline Tmem222 & transmembrane protein 222 \\
\hline Nhp2 & NHP2 ribonucleoprotein \\
\hline Hnrnpa2b1 & heterogeneous nuclear ribonucleoprotein A2/B1 \\
\hline Syt5 & synaptotagmin $\mathrm{V}$ \\
\hline Atp6ap1 & ATPase, $\mathrm{H}+$ transporting, lysosomal accessory protein 1 \\
\hline Paf1 & Paf1, RNA polymerase II complex component \\
\hline Mpc1 & mitochondrial pyruvate carrier 1 \\
\hline Ift20 & intraflagellar transport 20 \\
\hline Apip & APAF1 interacting protein \\
\hline Serinc1 & serine incorporator 1 \\
\hline Dynll1 & dynein light chain LC8-type 1 \\
\hline Arpp19 & cAMP-regulated phosphoprotein 19 \\
\hline Ube2d3 & ubiquitin-conjugating enzyme E2D 3 \\
\hline Mplkip & M-phase specific PLK1 intereacting protein \\
\hline Ndufa7 & NADH dehydrogenase (ubiquinone) 1 alpha subcomplex, 7 (B14.5a) \\
\hline Anp32e & acidic (leucine-rich) nuclear phosphoprotein 32 family, member $\mathrm{E}$ \\
\hline Farsa & phenylalanyl-tRNA synthetase, alpha subunit \\
\hline Sdhb & succinate dehydrogenase complex, subunit B, iron sulfur (Ip) \\
\hline Mrpl18 & mitochondrial ribosomal protein L18 \\
\hline Snap47 & synaptosomal-associated protein, 47 \\
\hline
\end{tabular}




\begin{tabular}{|l|l|}
\hline Bcas2 & breast carcinoma amplified sequence 2 \\
\hline Pdhb & pyruvate dehydrogenase (lipoamide) beta \\
\hline Ndufs3 & NADH dehydrogenase (ubiquinone) Fe-S protein 3 \\
\hline Faim2 & Fas apoptotic inhibitory molecule 2 \\
\hline Arpc2 & actin related protein 2/3 complex, subunit 2 \\
\hline Micu3 & mitochondrial calcium uptake family, member 3 \\
\hline
\end{tabular}




\section{The diphenylpyrazole compound anle138b blocks $A \beta$ channels and rescues disease phenotypes in a mouse model for amyloid pathology}

Ana Martinez Hernandez ${ }^{1,2, \dagger}$, Hendrik Urbanke ${ }^{1, \dagger}$, Alan L Gillman ${ }^{3, \dagger}$ (D), Joon Lee ${ }^{3, \dagger}$, Sergey Ryazanov ${ }^{4,5}$, Hope Y Agbemenyah ${ }^{6}$, Eva Benito ${ }^{1}$, Gaurav Jain ${ }^{1}$, Lalit Kaurani ${ }^{5}$, Gayane Grigorian ${ }^{7}$, Andrei Leonov ${ }^{4,5}$, Nasrollah Rezaei-Ghaleh ${ }^{4,8}$, Petra Wilken ${ }^{5,6,9}$, Fernando Teran Arce As, Jens Wagner $^{10}$, Martin Fuhrman ${ }^{10}$, Mario Caruana ${ }^{11}$, Angelique Camilleri ${ }^{11}$, Neville Vassallo ${ }^{11}$, Markus

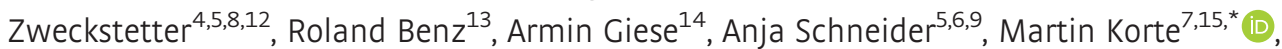

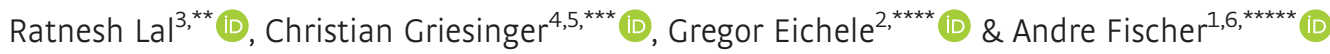

\begin{abstract}
Alzheimer's disease is a devastating neurodegenerative disease eventually leading to dementia. An effective treatment does not yet exist. Here we show that oral application of the compound anle138b restores hippocampal synaptic and transcriptional plasticity as well as spatial memory in a mouse model for Alzheimer's disease, when given orally before or after the onset of pathology. At the mechanistic level, we provide evidence that anle138b blocks the activity of conducting $A \beta$ pores without changing the membrane embedded A $\boldsymbol{A}$-oligomer structure. In conclusion, our data suggest that anle138b is a novel and promising compound to treat AD-related pathology that should be investigated further.
\end{abstract}

Keywords Alzheimer's disease; amyloid pathology; A $\beta$ channels; gene expression; membrane pores
Subject Categories Chromatin, Epigenetics, Genomics \& Functional Genomics; Neuroscience; Pharmacology \& Drug Discovery DOI 10.15252/emmm.201707825 | Received 23 March 2017| Revised 17 October 2017 | Accepted 20 October 2017

\section{Introduction}

Alzheimer's disease (AD) is the most common neurodegenerative disorder causing a severe emotional and economic burden to our societies. Due to increased life expectancies, the number of those afflicted with $\mathrm{AD}$ is expected to double by 2025. Despite intensive research, effective therapeutic approaches are still not available. The pathogenesis of $\mathrm{AD}$ has been linked to protein aggregation, namely the aggregation of amyloid-beta peptides $(A \beta)$ and tau protein. The accumulation of pathogenic aggregates of $A \beta$ peptides

\footnotetext{
Department for Epigenetics and Systems Medicine in Neurodegenerative Diseases, German Center for Neurodegenerative Diseases (DZNE), Göttingen, Germany

Department for Genes and Behavior, Max Planck Institute for Biophysical Chemistry, Göttingen, Germany

Department of Bioengineering, Materials Science and Engineering, Department of Mechanical and Aerospace Engineering and Institute of Engineering in Medicine, University of California San Diego, La Jolla, CA, USA

4 Department of NMR Based Structural Biology, Max Planck Institute for Biophysical Chemistry, Göttingen, Germany

5 DFG Research Center Nanoscale Microscopy and Molecular Physiology of the Brain, Göttingen, Germany

6 Department of Psychiatry and Psychotherapy, University Medical Center Göttingen, Göttingen, Germany

Department of Cellular Neurobiology, Technical University Braunschweig, Braunschweig, Germany

Department of Translational Structural Biology of Dementia, German Center for Neurodegenerative Diseases (DZNE), Göttingen, Germany

9 Group for Translational Research in Neurodegenerative Diseases, German Center for Neurodegenerative Diseases (DZNE) Göttingen, Göttingen, Germany

Group for Neuroimmunology and Imaging, German Center for Neurodegenerative Diseases (DZNE), Bonn, Germany

1 Department of Physiology and Biochemistry, Centre for Molecular Medicine and Biobanking, University of Malta, Msida, Malta

Department of Physiology and Biochemistry, Centre for Molecular Medicine and Biobanking, University of Ma

Department of Neurology, University Medical Center Göttingen, University of

Life Sciences and Chemistry, Jacobs University of Bremen, Bremen, Germany
Center for Neuropathology and Prion Research, Ludwig-Maximilians-University Munich, Munich, Germany

14 Center for Neuropathology and Prion Research, Ludwig-Maximilians-

Helmholtz Center for Infections Research, Braunschweig, Germany
†Present address:Departments of Medicine and Biomedical Engineering, University of Arizona, Tucson, AZ, USA

* Present address:Departments of Medicine and Biomedical Engineering, University of
*Corresponding author. Tel: +49 $5313913220 ;$ E-mail: m.korte@tu-braunschweig.de

**Corresponding author. Tel: +1858822 0384; E-mail: rlal@ucsd.edu

***Corresponding author. Tel: +49 551201 2201; E-mail: cigr@nmr.mpibpc.mpg.de

****Corresponding author. Tel: +49 551201 2701; E-mail: gregor.eichele@mpibpc.mpg.de

${ }^{* \star \star \star \star}$ Corresponding author. Tel: +49 551 3961211; E-mail: afische2@gwdg.de

These authors contributed equally to this work
} 
in the brain appears to be a key event in the pathogenesis of $\mathrm{AD}$ (Iversen et al, 1995; Tanzi, 2005; Jakob-Roetne \& Jacobsen, 2009; Goate \& Hardy, 2012), and targeting amyloid pathology still represents a promising therapeutic strategy (Haass \& Selkoe, 2007; Sevigny et al, 2016). The precise molecular events that trigger amyloid-induced decline of synaptic plasticity and neuronal cell death are still not entirely resolved and are likely to be multifactorial. One of the first explanations of neuronal dysfunction and toxicity in $\mathrm{AD}$ is the channel hypothesis first proposed by Arispe and coworkers (Arispe et al, 1993), which postulates that unregulated $A \beta$ ion channels result in a loss of ionic homeostasis (primarily through a gain of $\mathrm{Ca}^{2+}$ ) that eventually triggers neuronal dysfunction and cell death. In vivo evidence for this mechanism is, however, still rare, and a compound that would block pores and be active in mammalian animal models has not been reported yet. Thus, the original request by Arispe and coworkers that a useful strategy for drug discovery for treatment of $\mathrm{AD}$ should include screening compounds for their ability to block or otherwise modify $A \beta$ channels is still left unsatisfied (Arispe et al, 1993)

In this work, we examine the efficacy of the diphenylpyrazole (DPP) compound anle138b in an animal model of $A \beta$ deposition. Oral application of anle138b ameliorates $A \beta$-induced deficits in synaptic plasticity and memory formation. Using in vivo and in vitro approaches, we provide evidence that this effect is linked to the capacity of anle138b to reduce the conductivity of $A \beta$ pores in lipid bilayer membranes. Although other mechanisms likely contribute to this effect, our data suggest the functional modulation of the membrane bound $A \beta$-oligomers as a mechanism for neuroprotection and support the idea that anle138b should be taken into clinical trials to treat aggregopathies, including AD.

\section{Results}

Synaptic plasticity and memory function in a mouse model for deposition of amyloid $\beta$ peptides after oral treatment with anle138b

To initially test the potential of anle138b as therapeutic strategies to treat amyloid aggregation in Alzheimer's disease, we analyzed its effect in a Drosophila model for amyloid-induced neurotoxicity. We observed that treatment with anle138b improved survival times when compared to a vehicle-treated group (Appendix Fig S1). On the basis of these data, we decided to test the efficacy of anle138b in a mouse model for amyloid deposition. We like to state that none of the currently employed animal models for AD fully recapitulate the phenotypes seen in $\mathrm{AD}$ patients, and thus, care has to be taken when interpreting such data. In our study, we employed APPPS $1 \Delta 9$ mice (Jankowsky et al, 2001), a well-established model for ADlinked amyloid deposition. Since in the patients therapeutic intervention is normally initiated only after the onset of amyloid plaque formation, we decided to test anle138b in two experimental cohorts In the "pre-plaque group," treatment was initiated before the onset of pathology when mice were 2 months of age, while in the "postplaque group" treatment was initiated after the onset of amyloid deposition and memory disturbances in 6-month-old mice (Fig EV1 Jankowsky et al, 2004; Lalonde et al, 2005; Reiserer et al, 2007). In both cohorts, anle138b was continuously provided via food pellets.
Thus, in the pre-plaque group, mice were subjected to anle138b or placebo treatment from 2 months of age, and electrophysiological, behavioral, and biochemical analyses were initiated at 6 months of age. A group of wild-type mice (WT) treated with anle138b served as an additional control. We first measured synaptic plasticity by analyzing hippocampal long-term potentiation (LTP). While robust hippocampal LTP at the Schaffer collateral synapse was observed in WT control mice treated with anle138b (Fig 1A), LTP was significantly impaired in APPPS1 $\triangle 9$ mice that received placebo (Fig 1B). Notably, this LTP deficit was completely rescued in APPPS $1 \Delta 9$ mice treated with anle138b (Fig 1C). These data suggest that oral application of anle138b protects against $\mathrm{A} \beta$-induced impairment of hippocampal synaptic plasticity. To test whether the effect of anle138b on hippocampal plasticity also improved hippocampusdependent memory function, another group of anle138b and placebo-treated mice were subjected to the Morris water maze test, a well-established paradigm to assay spatial memory in rodents (Morris, 1984). Anle138b-treated WT mice displayed robust spatial learning as indicated by decreasing escape latency throughout the 8 days of training (Fig 1D). In contrast, APPPS $1 \Delta 9$ mice treated with placebo showed a significantly impaired escape latency (Fig 1D). This deficit was partially rescued in APPPS $1 \Delta 9$ mice that received anle138b. Spatial reference memory was analyzed in a probe test performed after 8 days of training. While WT mice showed a significant preference for the target quadrant, no such effect was observed in placebo-treated APPPS $1 \Delta 9$ mice (Fig 1E), confirming memory impairment in APPPS $1 \Delta 9$ mice. In contrast, anle138b-treated APPPS1 $\Delta 9$ mice displayed a significant preference for the target quadrant indicating restored spatial memory (Fig 1E). Swim speed was similar amongst the groups (Fig 1F). We also examined if anle138b would affect basal explorative behavior (Fig 1G) or basal anxiety (Fig 1H). No difference was found amongst the groups suggesting that oral administration of anle138b can protect APPPS $1 \Delta 9$ mice from deteriorating hippocampal synaptic plasticity and hippocampus-dependent memory consolidation.

Encouraged by these findings, we investigated whether anle138b could also reinstate synaptic plasticity and memory function when significant amyloid deposition had already occurred employing the post-plaque group (Fig EV1). To this end, 6-month-old APPPS $1 \Delta 9$ mice were treated with either anle138b or placebo for 4 months. Wild-type mice treated with anle138b served as an additional control group. Analysis was performed when mice were 10 months of age. In a first cohort, we measured hippocampal LTP. WT mice treated with anle138b showed robust LTP (Fig 2A), while LTP was significantly impaired in placebo-treated APPPS1 $\triangle 9$ mice (Fig $2 B$ ). Notably, a complete restoration of hippocampal LTP was seen in APPPS1 19 mice treated with anle138b (Fig 2C). In conclusion, similar to the pre-plaque group treatment with anle138b had a marked ameliorating effect on LTP even after the onset of plaque deposition.

To analyze whether reinstatement of hippocampal plasticity would also correlate with improved memory function, we subjected mice to the Morris water maze test. WT mice treated with anle138b rapidly learned the task as indicated by reduced escape latency throughout the 8 days of training (Fig 2D). Placebo-treated 10month-old APPPS1 $\triangle 9$ mice display impaired spatial learning as indicated by the escape latency that did not significantly decrease during the training (Fig 2D). When compared to the placebo group, 
A

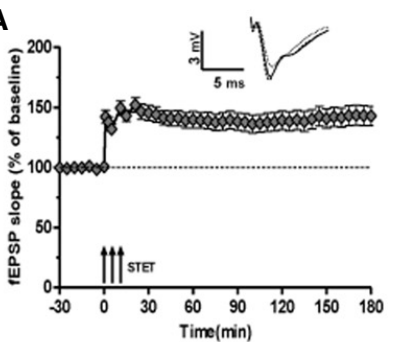

D

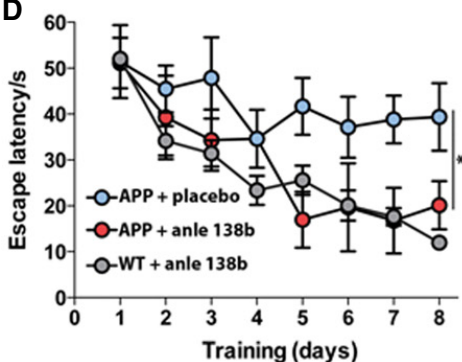

$\mathbf{F}$

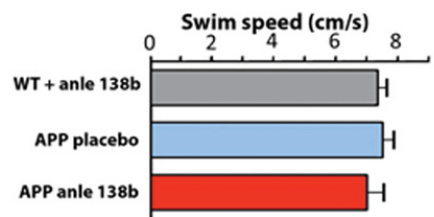

G

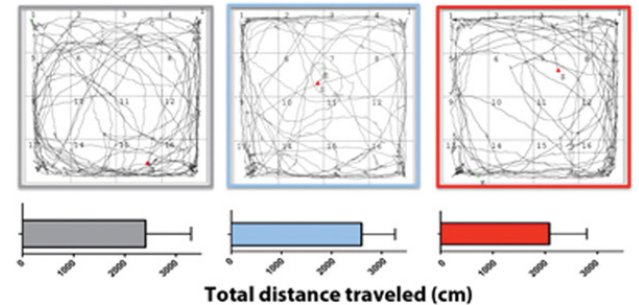

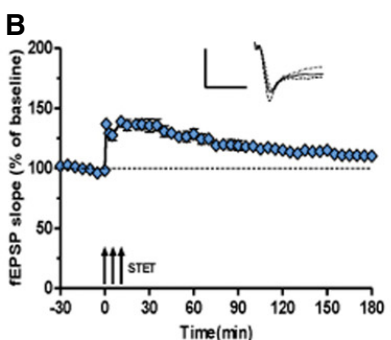
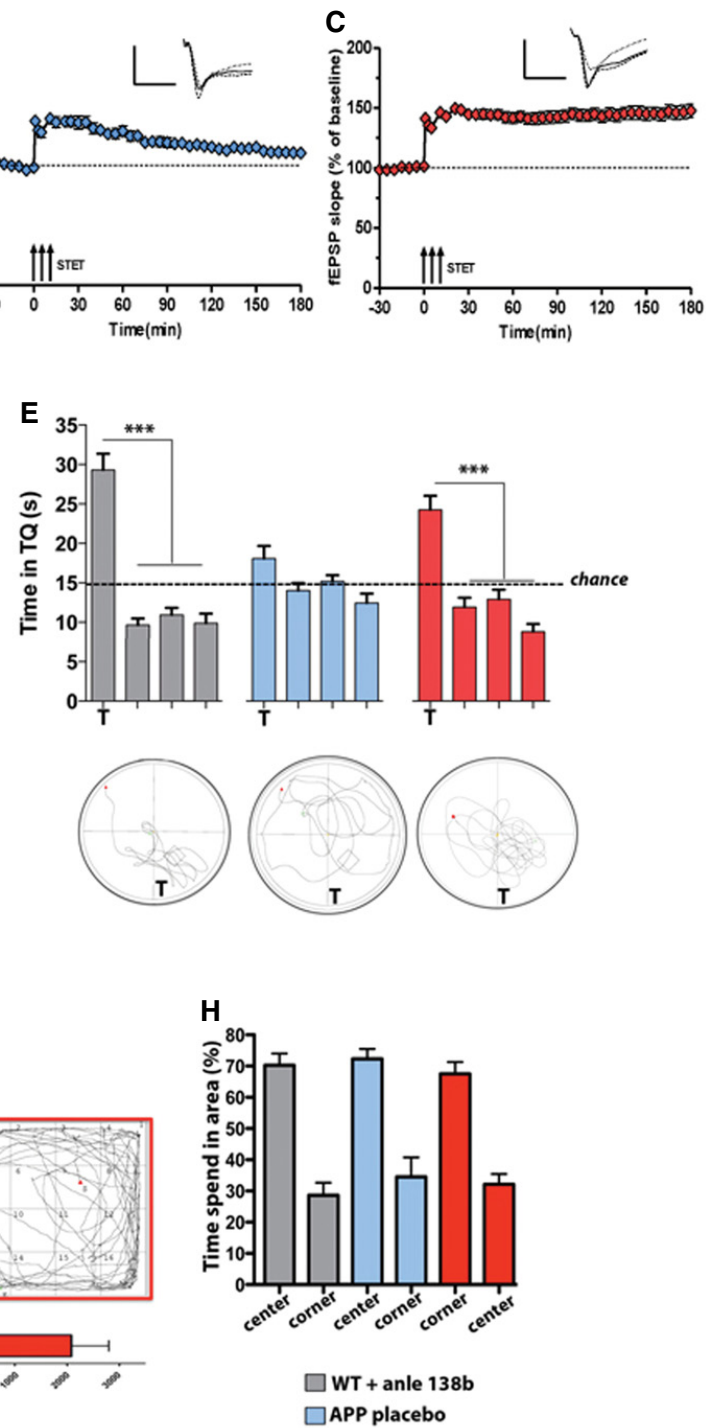

Figure 1. Anle138b rescues hippocampal LTP deficits spatial memory in the pre-plaque group.

A In wild-type mice of the pre-plaque group (treated from 2 to 6 months of age with anle138b), robust LTP that lasts for at least $3 \mathrm{~h}$ is elicited upon a strong tetanization (STET) (3 trains of 100 pulses at $100 \mathrm{~Hz}$ given $10 \mathrm{~min}$ apart, arrows) at the Schaffer collateral CA3-CA1 synapse ( $t$-test, $P=0.00005 ; n=16$ comparing before vs. after STET).

B LTP is not maintained in APPPS1 $\triangle 9$ mice treated with placebo. Here, the potentiation declined to baseline after $3 \mathrm{~h}$ ( $t$-test, $P=0.08 ; n=20$ comparing before vs. after STET).

C APPPS1 $\triangle 9$ treated with anle138b show a rescue of the LTP impairment ( $t$-test, $P=0.0001 ; n=23$ comparing before vs. after STET).

D Escape latency in the Morris water maze test is impaired in placebo- but not in anle138b-treated APP mice (one-way ANOVA $F=16.01,{ }^{* *} P=0.0008 ; n=15 /$ group).

E Probe test performed $24 \mathrm{~h}$ after the last training session. The lower panel shows representative swimming path during the probe test. $T=$ target quadrant compared vs. other quadrants ( $t$-test, ${ }^{* \star \star} P=0.00002 ; n=15 /$ group).

F Average swim speed during water maze training ( $n=15 /$ group).

G Explorative behavior in the open field test. Upper panel: Representative motion tracks during the test session. Lower panels show the total distance travelled during the 5 -min test session ( $n=15 /$ group).

Bar graph showing the time spent in the center vs. the corner of the open field ( $n=15 /$ group).

Data information: Error bars indicate SEM. 
APPPS $1 \Delta 9$ mice treated with anle138b showed improved spatial learning (Fig 2D). A probe test was performed after 8 days of training. WT mice treated with anle138b showed a significant preference for the target quadrant indicative of intact spatial reference memory (Fig 2E). Placebo-treated APPPS1 $\Delta 9$ mice exhibited severely impaired memory function (Fig 2E) and displayed no target preference (Fig 2E). In APPPS1 $\triangle 9$ mice treated with anle138b (Fig 2E), target preference was improved significantly but did not reach WT levels. Of note, swim speed was not different between the experimental groups (Fig 2F). Explorative behavior (Fig 2G) and basa anxiety (Fig $2 \mathrm{H}$ ) were measured in the open field test. There was no significant difference amongst groups. We also tested whether anle138b treatment would affect memory function in wild-type mice but did not observe any significant difference (Appendix Fig S2) Thus, oral administration of anle138b partially restores hippocampal plasticity and memory function in APPPS $1 \Delta 9$ mice even at an advanced stage of pathology.

\section{Anle138b reinstate transcriptional homeostasis and ameliorates amyloid pathology}

Pathological alterations often lead to aberrant changes in transcriptional plasticity indicating deregulated cellular homeostasis (Fischer, 2014a). In support of this, numerous studies demonstrated that AD pathogenesis correlates with altered gene expression in various brain regions (Fischer, 2014b; Benito et al, 2015; Matarin et al, 2015). Moreover, monitoring gene expression changes can inform about the efficacy of therapeutic intervention (Benito et al, 2015). In keeping with this idea, we performed RNA sequencing from hippocampal tissue dissected from mice in the pre- and post-plaque (Fig EV1) cohorts. Notably, there were no differences in hippocampal gene expression when comparing WT placebo- vs. WT anle138b-treated mice, suggesting that anle138b has no direct effect on transcriptome plasticity. Thus, we first compared gene expression in placebo-treated WT and placebo-treated APPPS1 $\triangle \mathrm{E} 9$ mice of the pre-plaque group. We identified 202 differentially expressed genes of which 73 were up- and 129 were down-regulated (Fig 3A Dataset EV1). Pathway analysis shows that down-regulated genes are linked to reduced energy metabolism, mitochondria function, cytoskeleton integrity, and synaptic plasticity, while pathways linked to cell growth were increased (Fig 3B). These data are in line with previous reports of gene expression changes in $\mathrm{AD}$ (Benito et al, 2015; Matarin et al, 2015) and were confirmed via qPCR (Appendix Fig S3A).

Because anle138b restored LTP and spatial memory functions in pre-plaque mice (see Fig 1), we hypothesized that the transcriptome of APPPS1 $\triangle \mathrm{E} 9$ mice treated with anle138b may more closely resemble that of wild-type mice. Indeed, a wild-type-like gene expression profile is to a large extent reinstated in anle138b-treated APPPS $\triangle \mathrm{E} 9$ and only 27 genes were deregulated when comparing wild-type mice treated with anle138b vs. APPPS $1 \Delta \mathrm{E} 9$ mice treated with anle138b groups (Fig 3A). Thus, none of the pathways deregulated in response to amyloid pathology (Fig 3B) remained significant after anle138b treatment. Accordingly, no enrichment for any specific pathway could be detected. The possibility remained that anle138b treatment may simply reduce the expression levels of the APP and PS1 transgenes specifically in APPPS1 $\triangle \mathrm{E} 9$. However, the RNA-sed data showed that APP and PS1 are increased in placebo-treated
APPPS1 $\triangle \mathrm{E} 9$ mice when compared to placebo-treated wild-type mice (see Dataset EV1). Similarly, APP and PS1 transgenes were elevated in anle138b-treated APPPS1 $\triangle \mathrm{E} 9$ mice compared to anle138b-treated wild-type mice (see Dataset EV1). These data were confirmed via qPCR showing that the expression of APP and PS1 transgenes is similar in placebo- and anle138b-treated APPPS1 $\triangle \mathrm{E} 9$ mice (Fig 3C); the gene expression data are in line with the electrophysiological and behavioral findings. It also shows that in the pre-plaque group, anle138b treatment reinstates cellular homeostasis in the hippocampus of APPPS1 $\triangle \mathrm{E} 9$ mice.

We next analyzed hippocampal gene expression in the postplaque group (Fig EV1). When comparing WT placebo and APPPS1 $\triangle \mathrm{E} 9$ placebo mice, we found 130 differentially expressed genes of which the majority (124) were up-regulated (Fig 3D), a finding confirmed via qPCR (Appendix Fig S3A). The comparison of anle138b-treated WT and anle138b-treated APPPS1 $\triangle \mathrm{E} 9$ mice revealed 220 differentially expressed genes, 207 up-regulated, and 13 downregulated (Fig 3D). Around half of these, 103 were also deregulated in the comparison WT placebo vs. APPPS1 $\mathrm{E} 9$ placebo (Fig $3 \mathrm{E}$ ), a finding confirmed via qPCR (Appendix Fig S3B). Pathway analysis revealed that gene expression changes almost exclusively represent an induction of neuroinflammatory processes (Fig 3F) in response to the APP and PS1 transgenes and this induction is not changed by treatment with anle138b. These data suggest that anle138b treatment does not have a major impact on inflammatory processes when given at a stage of advanced amyloid pathology. Since anle138b treatment nevertheless reinstated hippocampal synaptic plasticity and also partially restored memory function, these findings indicate that the therapeutic efficacy of anle138b is most likely not solely due to the dampening of amyloid-induced inflammation.

A previous study reported that anle138b exhibits therapeutic effect in TauP301S mice, a mouse model for Tau pathology (Wagner et al, 2015). Taking into account that Tau and amyloid pathology represent the two major causative factors for $\mathrm{AD}$, we wondered if anle138b would affect brain homeostasis as measured by hippocampal gene expression also in TauP301S mice. To this end, we obtained hippocampal brain tissue from the same experiment using anle138b or vehicle-treated wild-type and TauP301S mice as described by Wagner et al (2015). The experimental design employed by Wagner et al is similar to our pre-plaque group, since anle138b was feed to mice upon weaning. Similar to our data, feeding anle138b to wild-type mice had a neglectable effect on hippocampal gene expression when compared to vehicle fed wild-type mice (Appendix Fig S4, Dataset EV1). The comparison of vehicle-treated wild-type to vehicle-treated TauP301S mice revealed 257 differentially expressed genes that mainly represent increased pathways linked to neuroinflammation (Appendix Fig S4). Of note, this gene expression signature was significantly ameliorated in anle138b-treated TauP301S mice and only 16 genes were differentially expressed when comparing vehicle- vs. anle138b-treated TauP301S mice (Appendix Fig S4). These data allow for a number of interesting conclusions. First, at the hippocampal gene expression level, the overexpression of the human Tau gene carrying the P301S mutation leads to inflammatory response but in contrast to the data observed in APPPS1 $\triangle \mathrm{E} 9$ mice has very limited impact on the expression of genes linked to synaptic plasticity and learning and memory. Second, treatment with anle138b in TauP301S mice ameliorates the gene expression 
A

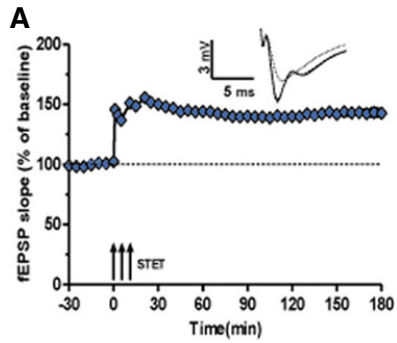

D
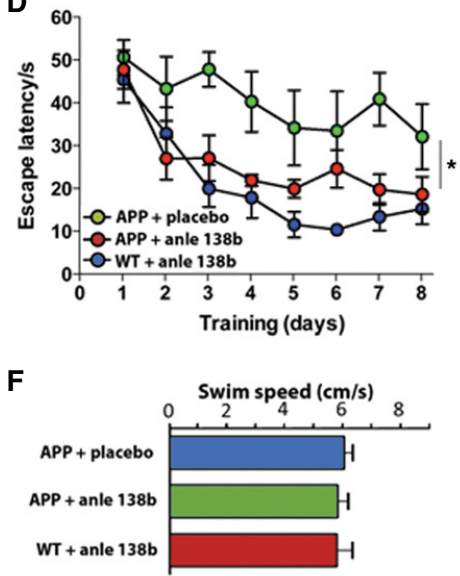

G

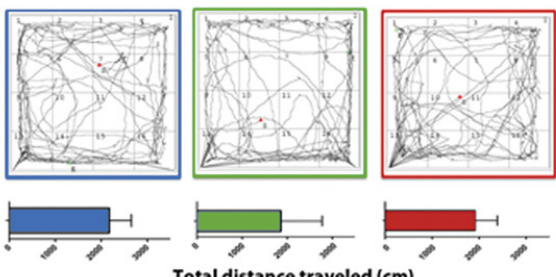

\section{B}

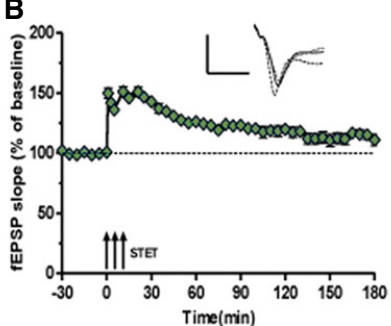

E
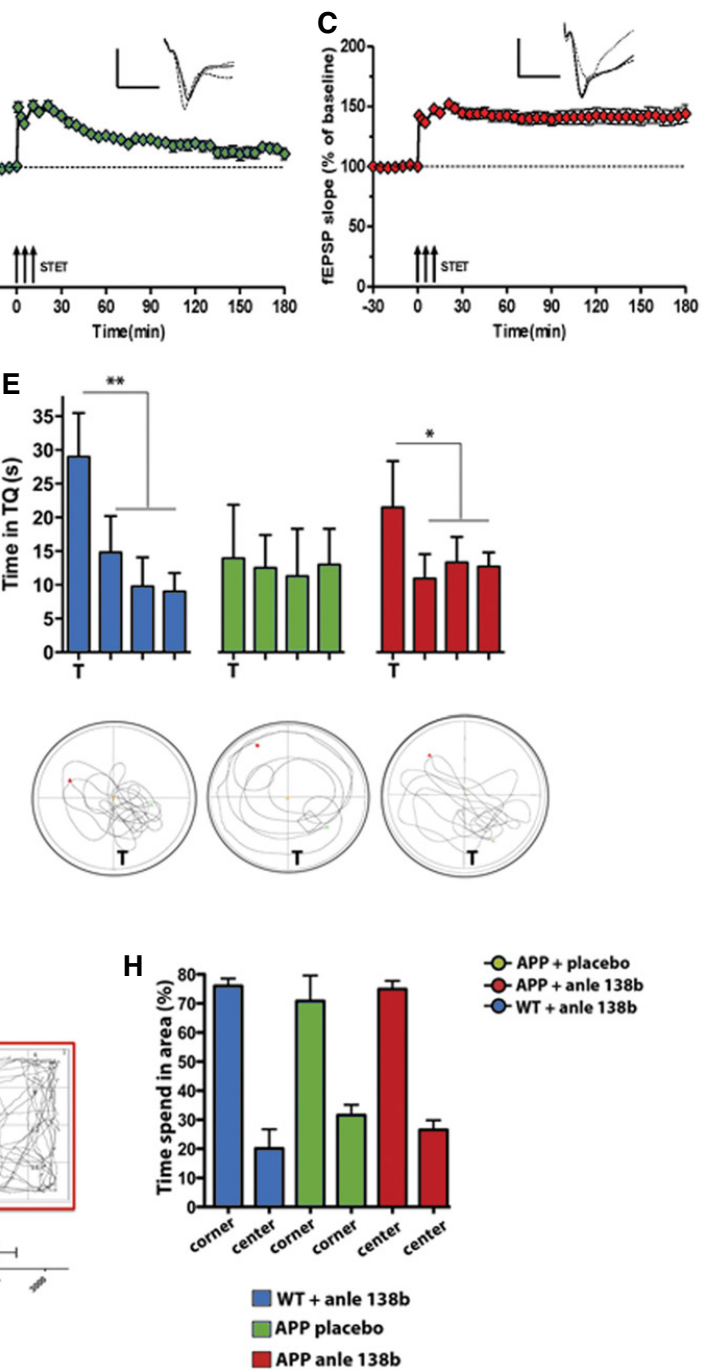

Figure 2. Anle138b rescues hippocampal LTP deficits and spatial memory in the post-plaque group.

A Wild-type mice of the post-plaque group (treated from 6 to 10 months of age) display robust LTP upon STET (arrows) that was maintained throughout the recording session ( $t$-test, $P=0.00006 ; n=30$ comparing before vs. after STET).

B Lasting LTP induced by STET was not observed in 10-month-old APPPS1 $\triangle 9$ placebo-treated mice. The potentiation decayed to baseline after $3 \mathrm{~h}(t$-test, $P=0.16$; $n=19$ comparing before vs. after STET).

C Treatment with anle138b starting at 6 months of age rescues LTP deficit in 10-month-old APPPS1 $\Delta 9$ mice ( $t$-test, $P=0.0003 ; n=20$ comparing before vs. after STET).

D Escape latency in the Morris water maze test is impaired in placebo-treated APP mice and partially restored to WT levels in anle138b-treated APP mice (one-way ANOVA, $F=35.94, P=0.00006$ * ${ }^{*} P=0.0309$ for APP + anle138b vs. APP + placebo and $P=0.4$ for APP + placebo vs. WT + anle138b; $n=7 /$ group)

E Probe test performed $24 \mathrm{~h}$ after the last training session. The lower panel shows representative swimming path during the probe test. $T=$ target quadrant compared vs. other quadrants ( $t$-test, ${ }^{\star \star} P=0.0007,{ }^{*} P=0.0031 ; n=7 /$ group).

F Average swim speed during water maze training $(n=7 /$ group $)$.

G Explorative behavior in the open field test. Upper panel: Representative motion tracks during the test session. Lower panels show the total distance travelled during the 5 -min test session ( $n=7 /$ group).

H Bar graph showing the time spent in the center vs. the corner of the open field ( $n=7 /$ group).

Data information: Error bars indicate SEM. In panels (A-C), triplets of arrows represent STET applied for inducing L-LTP. Insets in each graph represent typical fEPSP traces recorded $15 \mathrm{~min}$ before (dotted line), $30 \mathrm{~min}$ after (broken line), and $3 \mathrm{~h}$ after (full line) STET. 
A

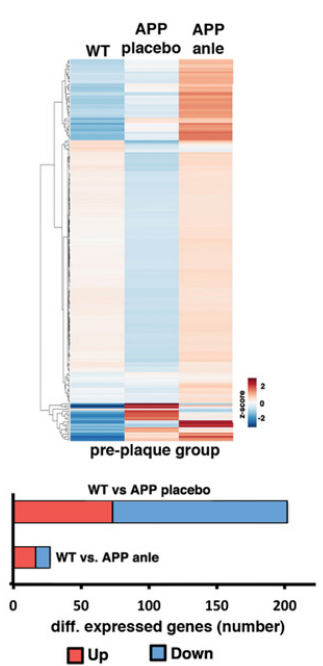

B
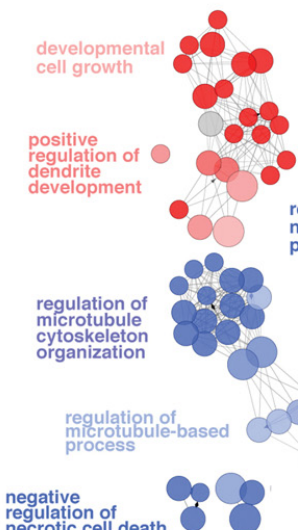

death

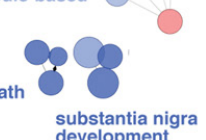

mitochondrial

transmembra

hydrogen
transport

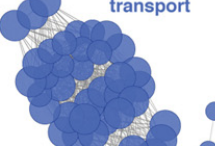
regulation of
neuronal synaptic plasticity

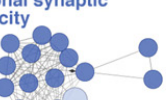

$\bullet$

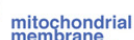

mitochondria
membrane
organization

astrocyte
differentiation

cellular modified
anmular
ân

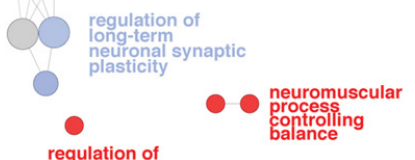

regulation of

regulain tyrosine
kinase activity
C
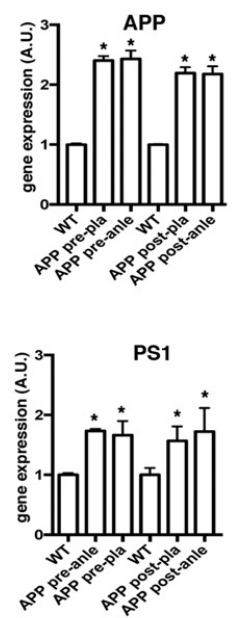

D $\begin{array}{cc}\text { APP } & \text { APP } \\ \text { placebo anle } & \end{array}$

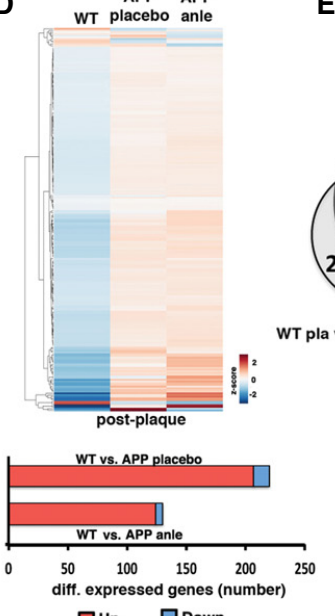

E
$\mathbf{F}$

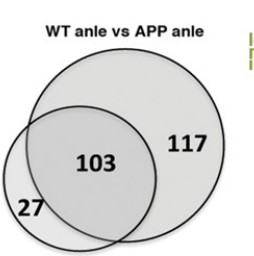

pla vs APP pla gquation

immunoglobulin
imedategd Im
response immune

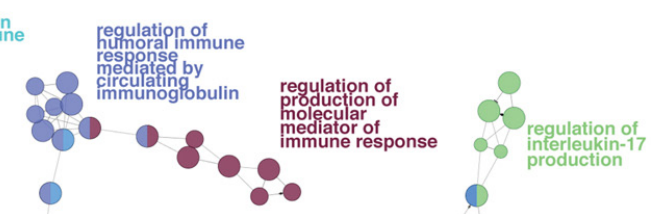

antigen
recentor-mediated
signaling pathway

10

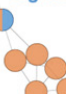

regulation of $\mathrm{B}$
cell activation
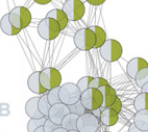

08

positive
regulation of

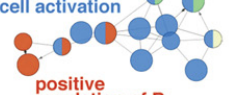

positive
regulation of $B$

000 immunune system

cell activation

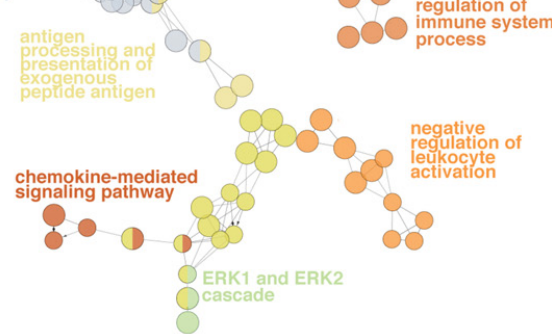

Figure 3. Hippocampal transcriptome analysis in anle138b-treated mice.

A Upper panel: Heat map showing differentially expressed genes in placebo-treated WT $(n=4)$, placebo-treated APPPS1 $\triangle$ E9 mice $(n=4)$, and anle138b-treated APPPS1 $\triangle$ E9 mice $(n=4)$ of the pre-plaque group. Note that a WT-like gene expression profile is to a large extent reinstated in APPPS1 $\triangle$ E9 mice upon treatment with anle138b. Lower panel: Bar graph showing the number of up and down-regulated genes.

B Pathways down-regulated (blue) or up-regulated (red) in 6-month-old APPPS1 $\triangle$ E9 mice when compared to age-matched WT controls (pre-plaque group; see Fig EV1).

C Expression of APP and PS1 genes in WT and APPPS1 1 E9 transgenic mice, placebo- or anle138b-treated in pre- and post-plaque groups. Note that anle138b treatment does not affect the expression level of APP or PS1. Post hoc analysis revealed a significantly increased expression of APP and PS1 in transgenic mice when compared to wild-type mice $\left({ }^{\star} P<0.05\right)$. Error bars indicate SEM.

D Upper panel: Heat map showing differentially expressed genes in placebo-treated WT $(n=4)$, placebo-treated APPPS1 $\Delta$ E9 mice $(n=5)$, and anle138b-treated APPPS1 $1 \Delta 9$ mice $(n=5)$ of the post-plaque group. Lower panel: Bar graph showing the number of up- and down-regulated genes.

E Venn diagram showing that 10-month-old mice treated with placebo or anle138b show very similar changes in hippocampal gene expression (post-plaque group; see Fig EV1).

F Pathway analysis based on the 103 genes commonly increased in placebo- and anle138b-treated APPPS1 $\triangle E 9$ mice at 10 months of age. Note that the pathways are exclusively linked to neuroinflammation. 
changes, at least when treatment is initiated before the onset of pathology, further confirming that treatment with anle138b helps to reinstate hippocampal homeostasis.

Given that anle138b was shown to counter aggregation in models for $\alpha$-synuclein and prion toxicity (Wagner et al, 2013), it is likely that at least part of the therapeutic effect observed in this study is due to anle138b interfering with amyloid aggregation. To test this hypothesis, we prepared brain slices for histochemical analysis. We used thioflavin $\mathrm{S}$, a common stain to quantify $\mathrm{A} \beta$ plaques (Fig $4 \mathrm{~A}$ ) We first assayed the amyloid plaques pathology in the hippocampus and cortex in the pre-plaques group. Since no amyloid pathology was detectable in WT mice, these mice served as negative control. We observed a significant reduction in the number of plaques and the total area covered by plaques in anle138b-treated mice (Fig 4A and $\mathrm{B})$. Similar results were obtained when we analyzed plaque load in the post-plaques group (Fig 4B and C), although the pathology was generally more severe in the post-plaque group. Thus, oral administration of anle138b reduces amyloid pathology when given before or after the onset of pathology.

\section{Biophysical characterization of $A \beta$ in the presence of anle138b}

To better understand the mechanisms that underlie the therapeutic effect of anle138b, we analyzed its impact on $A \beta$-induced pore formation (Arispe et al, 1993). To this end, we employed black lipid membranes (BLM) using a mixture of POPE and DOPS in a 1:1 ratio or oxidized cholesterol/n-decane, an assay in which the current passing through a membrane is a measure of membrane integrity (Appendix Fig S5A). We first established that the conductance and the morphology of POPE and DOPS lipids, as measured by atomic force microscopy (AFM), lipid bilayers are not affected in the presence of anle138b or $A \beta$ peptides (Appendix Fig S6).

When we examined the effect of $A \beta_{1-42}$ in DOPS/POPE (1:1) membranes, we observed a stepwise growth of bulk membrane conductance. Such activity is indicative of the combined action of many individual pores (Fig 5A). Pore "stacking" is likely the result of prolonged open lifetimes and the formation and/or opening of additional conducting pores. Anle138b-doped membranes demonstrated fewer simultaneously active pores and significantly reduced bulk conductance (Fig 5A and B, Appendix Fig S5) compared to membranes lacking the anle138b compound (Fig 5A and B, Appendix Fig S5). Our results indicate that treatment with anle138b alters the pore dynamics, resulting in less stable and shorter lived "open" pores. Decreased pore stability leads to a reduction in the total number of simultaneously conducting pores and significantly decreased conductance across the membrane. AFM data revealed that anle138b treatment did not affect the surface structure of $A \beta_{1-42}$ pores (Fig EV2), suggesting that anle138b does not simply prevent $\mathrm{A} \beta_{1-42}$ from entering lipid bilayer membranes and forming pores Rather anle138b appears to render conducting $A \beta$ pores to nonconducting ones-likely through structural change to the membrane embedded region of $A \beta_{1-42}$-thereby providing one possible mechanism by which anle138b ameliorates LTP and learning deficits in APPPS1 1 E9 mice. Similar effects were observed when the conductance measurements were repeated in oxidized cholesterol (Appendix Fig S5B).

To provide in vivo evidence for pore formation, we treated primary hippocampal neurons with $A \beta_{1-40}$ monomers or oligomers in the absence or presence of anle138b and assayed membrane integrity. While the addition of $A \beta_{1-40}$ oligomers but not $A \beta_{1-40}$ monomers significantly damaged membrane integrity, this effect was reversed by anle138b (Fig 5C). This restoration is not simply due to an anle138b-mediated reduction in cell death, since the cell viability of hippocampal neurons measured in the MTT assay was identically affected by $A \beta_{1-40}$ monomer and oligomer treatment in our experimental setting (Fig 5D). In this context, it is important to state that different protocols for $A \beta$ treatment of neurons have been used to study various effects of $A \beta$ on neuronal plasticity and integrity. The concentration of $A \beta$ oligomers employed in our study is within the range of these studies. Nevertheless, such data always need to be interpreted with care since a cell culture system cannot not fully recapitulate the situation observed in human patients. Interestingly, anle138b treatment was also able to ameliorate the effect of toxic $A \beta$ species on membrane integrity if added after neurons had been incubated with $A \beta$ oligomers and already exhibited impaired viability, which is in line with our data showing that anle138b completely ameliorated LTP and partially restored memory defects in the post-plaque group (Appendix Fig S7A and B). We also employed another experimental system to test the effect of $A \beta_{1-42}$ oligomers and anle138b on biological membranes. To this end, we employed the cytochrome release assay (CRA) on isolated mitochondria that were exposed to either $\alpha$-synuclein, tau, or $A \beta_{1-42}$ oligomer in the presence or absence on anle138b. Our data reveal a substantial damage to mitochondrial membranes in all conditions that is attenuated by anle $138 \mathrm{~b}$ (Appendix Fig S7C), suggesting that anle138b has general effect on membrane integrity.

\section{Discussion}

In this work, we investigated the effect of anle138b in the established APPPS1 $\triangle \mathrm{E} 9$ mouse model of $\mathrm{AD}$. The APPPS1 $\mathrm{E} 9$ mouse model is characterized by dysfunction of neurons and detected by memory decline after 4 months and severe plaques formation after 6 months (Kummer et al, 2014). The most important findings of the present study were that anle138b treatment completely restored hippocampal LTP in the pre- and post-plaque groups of APPPS1 $\triangle \mathrm{E} 9$ mice. In line with this observation, spatial reference memory was fully or partially restored in the pre- and post-plaque groups, respectively. There have been numerous pre-clinical studies aiming to restore synaptic plasticity and memory function in mouse models for amyloid pathology. These range from therapeutic approaches that aim to modulate causative factors including A $\beta$-toxicity via antibody-based therapies (Selkoe \& Hardy, 2016), small molecules that modulated APP processing (Vassar et al, 2014), small molecules that are thought to target toxic amyloid species directly (Doig \& Derreumaux, 2015) to symptomatic treatments not directly targeted toward amyloids (Fischer, 2016). Anle138b belongs to the first category. Its therapeutic effect resembles that of other small molecule drugs. For example, epigallocatechin gallate (EGCG) was shown to affect $A \beta$ toxicity by redirecting toxic $A \beta$-structures into off-pathway oligomers (Ehrnhoefer et al, 2008; Bieschke et al, 2010). This agent was able to ameliorate spatial memory deficits in APP mice after the onset of amyloid deposition (Liu et al, 2014). In this context, it is interesting to note that when administered via dry food anle138b 
A

\section{Pre-plaque group}

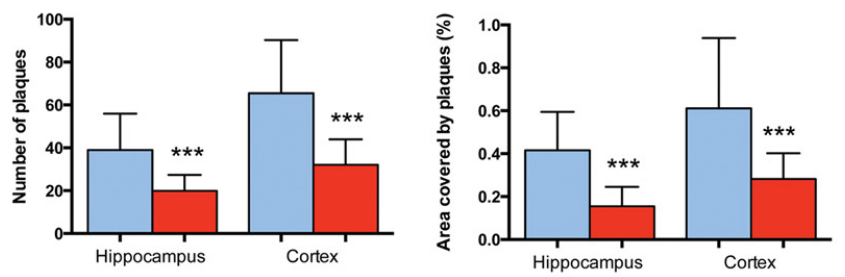

B

Post-plaque group
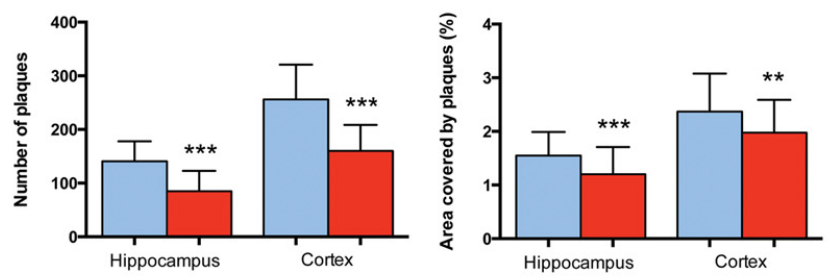

C

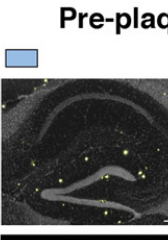

Pre-plaque group

Post-plaque group
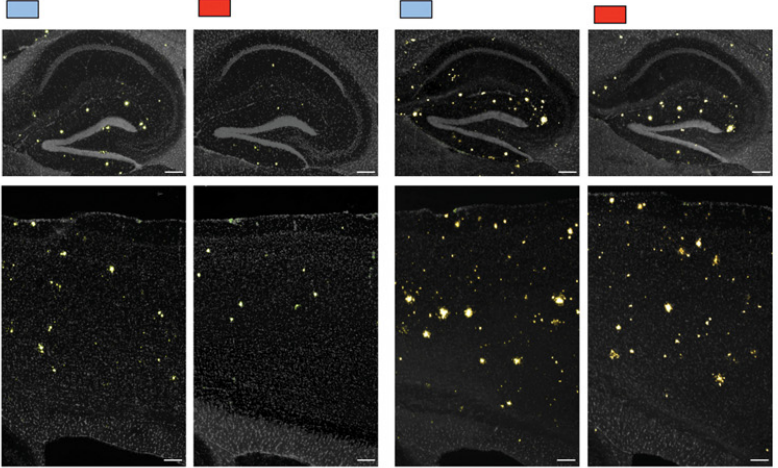

$\square$ APP placebo $\square$ APP anle 138b

Figure 4. Amyloid plaque pathology is ameliorated by anle138b treatment in the pre-plaque (A) and post-plaque (B) groups.

A Reduced amyloid pathology as indicated by reduced number of plaques (left panel) and reduced area covered by plaques (right panel) in the pre-plaque group ( $t$-test ${ }^{* * *} P=0.00003 ; n=5 /$ group $)$.

B Reduced amyloid pathology as indicated by reduced number of plaques (left panel) and reduced area covered by plaques in the post-plaque group ( $t$-test

${ }^{\star \star *} P=0.00007,{ }^{\star *} P=0.005 ; n=5 /$ group $)$.

C Representative images showing thioflavin S staining in the hippocampus and cortex of mice of the pre- and post-plaque groups. Scale bar $=200 \mu \mathrm{m}$. Data information: Error bars indicate SEM.

was previously found to be taken up to reach levels of $100 \mu \mathrm{M}$ in the brain (Wagner et al, 2015). Anle138b metabolites are very hydrophilic, and they were detected in multiple organs but were absent in brain (Wagner et al, 2013). This indicates that anle138b is not metabolized in the brain. Anle138b was also found to be non-toxic in mice up to a dose of $2 \mathrm{~g} / \mathrm{kg}$. Mice receiving similar concentrations of anle138b as used in our study lived without any detectable toxicity, even when the drug was given longer than a year (Wagner et al, 2015). Also in our study, no negative or positive effects of anle138b on WT mice were observed. 
A
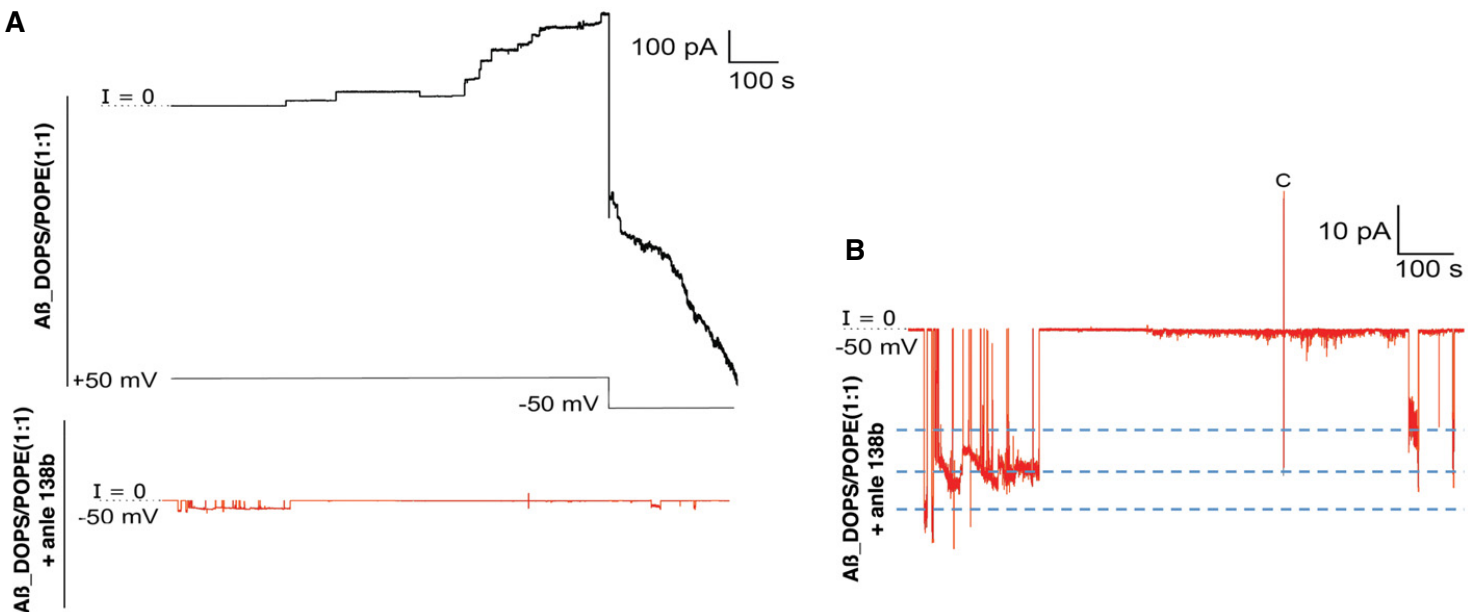

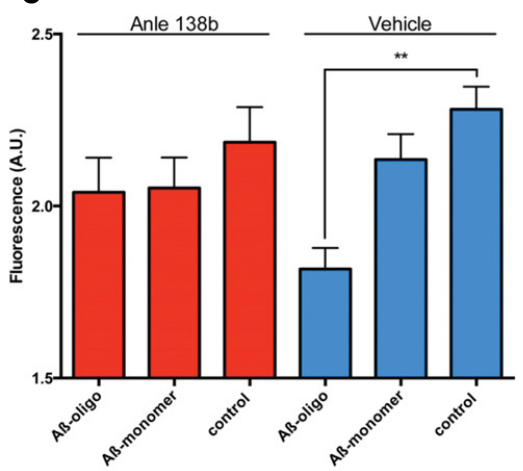

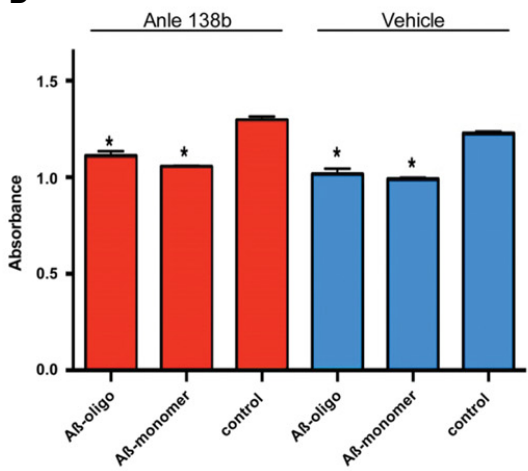

E

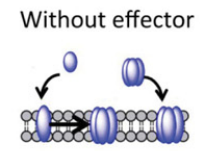

With anle138b

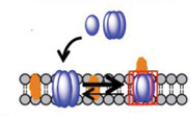

(1) A $\mathrm{A}$ conducting pore

(20) A non-conducting pore

anle138b

Figure 5. Anle138b ameliorates $A \beta_{1-40}$ and $A \beta_{1-42}$-induced membrane integrity.

A $\quad A \beta_{1-42}$ induces pore-like step ionic current increases across lipid bilayer membranes and grows with inverted sign after voltage inversion to amplifier saturation current. In the presence of anle138b, the current remains at low amplitude and does not increase beyond $30 \mathrm{pA}$.

B Expanded trace of anle138b-treated membrane presented in (A). Discreet conductance levels are highlighted suggesting multiple opening and closing events for three individual pores.

C Hippocampal neurons (DIV 10) were treated with anle138b (1 $\mu \mathrm{M})$ or vehicle before $A \beta_{1-40}$ oligomers or monomers were added (10 $\left.\mu \mathrm{M}, n=4 / \mathrm{group}\right)$. After $48 \mathrm{~h}$, membrane integrity was measured as fluorescence intensity using a CyQUANT assay (Thermo Fisher). In the vehicle group, membrane integrity was significantly impaired when treated with $A \beta$ oligomers comparing with control neurons or $A \beta$ monomer-treated neurons, anle138b-treated neurons did not exhibit a difference between addition of $A \beta$ monomers or $A \beta$ oligomers ( $t$-test, $\left.{ }^{*} *=0.007\right)$.

D Same experimental setting as in (C) but cell viability was measured using the MTT assay ( $t$-test, ${ }^{*} P=0.002$ vs. control). No difference in cell viability was observed for $A \beta$ monomer or oligomer treatment in the absence or presence of anle138b.

E Schematic of potential mechanisms of activity inhibition for anle138b. In the absence of anle138b, A $\beta$ monomers and/or oligomers insert in the membrane and form conducting pores. Treatment with anle138b renders these conductive channels inactive most probably due to reduced life time of the open state.

Data information: Error bars indicate SEM.

In addition to the restoration of hippocampal LTP and spatial reference memory, we also observed restoration of physiological, wild-type-like gene expression profile in the pre-plaque group, indicating that hippocampal cells reinstate homeostasis. In APPPS $1 \Delta \mathrm{E} 9$ mice that received normal chow, genes linked to metabolic function and neuronal plasticity were markedly down-regulated. Feeding with anle138b-containing chow completely reversed this effect on gene expression. Even in the placebo-treated pre-plaque group, we observed little evidence for neuroinflammatory processes, which is in contrast to the data from the post-plaque group. In fact, the gene expression changes observed in the placebo-treated post-plaque group were dominated by increased inflammation. These data suggest that in APPPS $1 \Delta \mathrm{E} 9$ mice, the decline of synaptic plasticity precedes inflammatory processes. Of note, anle138b treatment had 
no effect on pathological gene expression pattern in the post-plaque group, yet hippocampal LTP was completely restored and spatial memory was partially restored. These data suggest that therapeutic strategies that aim to reduce amyloid toxicity-at least in the APPPS1 $\triangle$ E9 model - may have little influence on neuroinflammation when applied at an advanced stage of the disease. Nevertheless, a significant therapeutic effect could be observed in the post-plaque group. This might be explained by removal of toxic $A \beta$ species which can lead to the restoration of synaptic function in neurons even in a detrimental inflammatory environment. These data are highly interesting, since one argument for the failure of clinical trials targeting amyloid deposition or modulation of $A \beta$ cleavage is that such treatments are ineffective when given at an advanced stage of the diseases. Our data suggest, however, that anle138b targets $A \beta$ related pathological events that allow recovery of synaptic function even if secondary pathological events such as inflammation persist.

The finding that anle138b treatment ameliorates synaptic plasticity and learning deficits in APPPS1 $1 \mathrm{E} 9$ is of utmost importance. These data are in line with previous observations showing that the same compounds have beneficial effects in animal models for Creutzfeld-Jakob, Parkinson's disease, and Tau pathology. Specifically, the latter finding is intriguing, since Tau and $A \beta$ pathology are believed to be the main causative factors of $\mathrm{AD}$ pathogenesis. While Wagner et al (2015) showed that anle138b in Tau P301S mice rescues synaptic and neuronal loss, we could further substantiate these data by showing that anle138b treatment also ameliorates defects in hippocampal transcriptome plasticity in the same Tau P301S mice. Taken together, these data suggest the revealing possibility that anle138b is able to ameliorate Tau and $A \beta$ pathology. Therefore, to the best of our knowledge, anle138b would be the first small molecule that has a direct effect on the two major hallmarks of $\mathrm{AD}$.

These data are also important from a translational point of view. Taking into account that the various animal models for AD only recapitulate part of the pathogenesis observed in human patients, it is intriguing that anle138b was able to rescue disease progression in various different $\mathrm{AD}$ models and models for aggregopathies, raising the hope that anle138b could also be effective in human patients.

The mechanism by which toxic $A \beta$ species induce synaptic dysfunction and neurotoxicity is still unresolved. Proposed mechanisms include NMDA receptor endocytosis (Shankar et al, 2007), $\alpha$ amino-3-hydroxy-5-methyl-4-isoxazole propionic acid surface receptor modulation (Querfurth \& LaFerla, 2010), and A $\beta$ pore formation (Arispe et al, 1993). Moreover, the presence of amyloid plaques was shown to change structural plasticity of neurons (Spires \& Hyman, 2004). Nevertheless, amyloid plaques are unlikely the sole reason for disturbed synaptic function and memory consolidation in $\mathrm{AD}$ and it has even been suggested that they present a compensatory mechanism of the brain in order to deal with toxic $A \beta$ species (Selkoe \& Hardy, 2016). This is further supported by our findings that anle138b treatment in the post-plaque group was able to restore hippocampal LTP and spatial reference memory, although the plaque load was even higher when compared to placebo-treated $\mathrm{APPPS} 1 \triangle \mathrm{E} 9$ mice of the pre-plaque group suggesting that the reduction in amyloid plaque load cannot be the main mechanisms by which anle138b exerts its therapeutic action. The pore formation hypothesis has long been proposed but tested experimentally to a very limited degree, which is also due to the fact that an array of various in vitro and in vivo methods needs to be combined to address this issue. We focused on the question whether anle138b modulates the pore forming activity of $A \beta$ because synaptic function relies on the integrity of membranes and their ability to modulate ion fluxes in a voltage-dependent way. This pore-based mechanism has been envisaged to underlie impaired neuronal function and cell death (Arispe et al, 1993). Modulation of pore formation was also implicated in cell-based models for EGCG mode of action (Diaz et al, 2009) and of its congeners MRS2481 and MRS2485 (Diaz et al, 2009). The observed stepwise growth of bulk membrane conductance in the presence of $A \beta$ (without anle138b) indicates that activity increases through the combined action of many individual pores.

Numerous molecular dynamics (MD) simulations (Jang et al, 2008; Arce et al, 2011; Gillman et al, 2014) and NMR spectroscopybased investigations (Sarkar et al, 2014) of $\mathrm{A} \beta$ pores have pointed to a $\beta$-barrel structure for the intramembrane region of the pore. It was previously shown that $A \beta$ containing a point substituted proline (F19P) showed pore structure by AFM but did not demonstrate ionic conductance (Capone et al, 2012; Connelly et al, 2012b). The chemical structure of proline introduces a "kink" in the peptide's secondary structure, which is known to disrupt $\beta$-sheet formation. MD simulations of F19P barrel structure showed that $\beta$-sheet destabilization led the highly charged N-terminal regions to bind at the peptide mouth and collapsed the pore (Umehara et al, 2010; Connelly et al, 2012a). Our data are consistent with such a model and suggest that anle138b induces a conformational change within $\mathrm{A} \beta$ pores that greatly reduces or, in most pores eliminates, ionic flux. Steric blockage by anle138b without a conformational change of the pore is energetically unfavorable due to the hydrophobicity of anle138b, as this scenario would require anle138b to be in contact with water molecules inside the pore. The above-mentioned mechanisms provide further insight into the method of action in preventing pore activity and reducing Alzheimer's pathogenicity (Fig 5E).

It has to be mentioned, however, that all of the above-described experiments are based on in vitro approaches using lipid bilayers. In vivo evidence further supporting the notion that anle138b counteracts the detrimental effect of toxic $A \beta$ species on membrane integrity stems from our finding that $A \beta_{1-40}$ administration to cultured hippocampal neurons compromised membrane integrity, an effect which was attenuated by anle138b. Our interpretation that conversion of conducting to non-conduction $\mathrm{A} \beta$ pores is one possible mode of anle138b action. It may also explain that anle138b ameliorated all tested disease phenotypes in the pre-plaque group, while in the post-plaque group only LTP was completely restored. Hence, in the post-plaque group, the presence of toxic $A \beta$ species may already have induced secondary processes such as inflammation that persistently affects memory function even if membrane integrity and LTP are restored in neurons. Yet, we like to reiterate that while our data point to the existence of $A \beta$ pores in vivo, a definite proof is still missing. Further evidence in support of the $A \beta$ pores stems from a study in which $A \beta$ oligomers induce single-channel calcium fluorescence transients in Xenopus oocytes (Demuro et al, 2011). Nevertheless, we cannot exclude that anle138b affects other cellular processes than $A \beta$-mediated conducting pores in membranes. Thus, it is also possible for example that in vivo $\mathrm{A} \beta$ sequesters membrane lipids, thereby affecting membrane integrity, which is then prevented by the action of anle138b. 
In conclusion, our data show that anle138b can reinstate synaptic plasticity and memory function in a mouse model for amyloid pathology via mechanisms that-at least in part-appear to involve the blockage of $\mathrm{A} \beta$-induced pores in membranes. Careful analysis of this activity indicates that the oligomers are still in the membrane, but pores have a changed conductivity profile, mainly staying open for shorter time and lacking the possibility of building up large currents as seen when anle138b was absent. Taking into account that anle138b was also shown to ameliorate disease phenotypes in a mouse model for Tau pathology, thus being to the best of our knowledge one of the first compounds that seems to causatively interfere with both of the two major hallmarks of $\mathrm{AD}$, we suggest that anle138b to further be validated in clinical trials for its potential to treat $\mathrm{AD}$ and perhaps other aggregopathies.

\section{Materials and Methods}

\section{Mouse experiments}

The APP(Swe)/PS1EA9 (henceforth called: APPPS1 $\Delta \mathrm{E} 9$ ) mouse model of $\mathrm{AD}$ was used for this study. The age of the animals is indicated for each experiment. In brief, mice of the pre-plaque group were analyzed at 6 months of age, while mice of the post-plaque group were 10 months of age. Upon completion of treatment, these and control mice underwent cognitive assessment by a behavior battery of tests. Electrophysiology, RNA sequencing, and histochemical analysis of plaque burden were also assessed. All mice were male and were maintained on a C57BL/6 background. They were kept in a 12-h dark/light cycle and housed in groups under constant standard conditions of temperature and humidity. Mice had ad libitum access to food and water. Animal care and handling were carried out in compliance with the Declaration of Helsinki and approved by local ethical committees.

\section{Anle138b treatment}

In order to investigate the prophylactic effect of anle138b, we treated healthy, plaque-free, adult, APPPS $1 \triangle 9$ mice with placeboor anle138b-containing dry food pellets for 4 months from 2 to 6 months of age (pre-plaque group; Fig EV1). Age- and sexmatched wild-type littermates were also treated and served as controls. Similarly, in order to investigate the therapeutic effect of anle138b, we treated symptomatic APPPS1 $\triangle \mathrm{E} 9$ mice and treated them for 4 months from 6 to 10 months of age (post-plaque group; Fig EV1). Controls were age- and sex-matched wild-type littermates treated with anle138b or placebo. Anle138b was administered orally. Dry food pellets were prepared containing $2 \mathrm{~g}$ anle138b per $\mathrm{kg}$ food (SSNIFF). This amounted to an estimated daily dose of $500 \mathrm{mg} / \mathrm{kg}$ (at an approx. 6-g daily food consumption and a 25-g average body weight). Based on pharmacokinetic studies, 40-70 $\mu \mathrm{M}$ anle138b reached the brain during most of the wake phase (Wagner et al, 2015). Placebo food was prepared from the same batch but without anle138b (SSNIFF). Of note, our previous PK studies in mice have shown that after a single bolus the halflife of anle138b in the brain is approximately $4 \mathrm{~h}$ (Wagner et al, 2013).

\section{Morris water maze (MWM)}

Mice were single-caged and brought into the testing room at least 1 week prior to the beginning of the experiment to allow them to acclimate. In the MWM, mice were trained to find a submerged $10 \times 10 \mathrm{~cm}$ platform in a pool $(1.10 \mathrm{~m}$ in diameter $)$ of milky water using spatial cues by the pool sides as orientation points. Mice were trained over 8 consecutive days with four trials per day per mouse. Time and path to platform were tracked and recorded (TSE systems). On day 9 (probe test), the platform was removed and quadrant preference was recorded (target quadrant being the one where the platform was previously located).

\section{Open field}

Mice were allowed to spend $5 \mathrm{~min}$ in an open arena $(40 \times 40 \mathrm{~cm})$. Path length while exploring and time in the center or corners were quantified using the VideoMot2 System (TSE).

\section{RNA sequencing}

Library preparation and cluster generation for mRNA sequencing were performed according to Illumina standard protocols (TruSeq, Illumina). Libraries were quality-controlled and quantified using a Nanodrop 2000 (Thermo Scientific), Agilent 2100 Bioanalyzer (Agilent Technologies), and Qubit (Life Technologies). Data will be made available upon publication via GEO (accession number GSE104424). Base calling from raw images and file conversion to fastq-files was achieved by Illumina pipeline scripts. Subsequent steps included quality control (FastQC, https://www.bioinformatics. babraham.ac.uk/projects/fastqc/), mapping to reference genome (mm10, STAR aligner v2.3.0 (Djebali et al, 2012) non-default parameters), read counting on genes or exons (HTSeq, http://wwwhuber.embl.de/users/anders/HTSeq, mode: intersection-nonempty), and differential gene (DESeq2_1.4.5; Love et al, 2014) usage biostatistical analysis. PCA and distance heat maps were generated in $\mathrm{R}$ following instructions in the vignette for DESeq2. Genes were considered differentially expressed with an adjusted $P$-value lower than 0.05 (Benjamini-Hochberg). Gene set overlaps were calculated using Venny (http://bioinfogp.cnb.csic.es/tools/ve nny/). Pathway analysis was performed using Cytoscape (www.cy toscape.com) and Cytoscape extension ClueGO.

\section{Electrophysiology on hippocampal slices}

\section{Slice preparation}

Acute hippocampal slices prepared from WT mice and APPPS1 $\triangle \mathrm{E} 9$ mice were used for electrophysiological recordings. All the procedures were carried out in compliance to the guidelines from the Animal Committee on Ethics in the Care and Use of Laboratory Animals of TU-Braunschweig. Briefly, after anesthetization using $\mathrm{CO}_{2}$, the mice were decapitated and the brains were quickly removed and cooled in $2-4^{\circ} \mathrm{C}$ artificial cerebrospinal fluid (aCSF). The hippocampi were dissected, and transverse hippocampal slices $(400 \mu \mathrm{m})$ were prepared by using a manual tissue chopper. Then, slices were incubated for $3 \mathrm{~h}$ at $32^{\circ} \mathrm{C}$ in an interface chamber (Scientific System Design), which was continuously perfused with 
oxygenated aCSF at a flow rate of $0.8 \mathrm{ml} / \mathrm{min}$. The aCSF contained the following (in mM): $124 \mathrm{NaCl}, 4.9 \mathrm{KCl}, 1.2 \mathrm{KH}_{2} \mathrm{PO}_{4}, 2.0 \mathrm{MgSO}_{4}$, $2.0 \mathrm{CaCl}_{2}, 24.6 \mathrm{NaHCO}_{3}, 10 \mathrm{D}$-glucose, equilibrated with $95 \%$ $\mathrm{O}_{2}-5 \% \mathrm{CO}_{2}(32 \mathrm{~L} / \mathrm{h})$

\section{Slice recordings}

To evoke field EPSP (fEPSP) from Schaffer collateral/commissuralCA1 synapses, one monopolar lacquer-coated, stainless-steel electrode (5 M $\Omega$; AM Systems) was positioned at the stratum radiatum layer of the CA1 region. For recording fEPSP (measured as its initial slope function), one electrode (5 M 2 ; AM Systems) was placed in the CA1 apical dendritic layer and signals were amplified by a differential amplifier (Model 1700, AM Systems). The signals were digitized using a CED 1401 analog-to-digital converter (Cambridge Electronic Design). After the preincubation period, an input-output curve (afferent stimulation vs. fEPSP slope) was generated. Test stimulation intensity was adjusted to elicit fEPSP slope of $\sim 40 \%$ of the maximal fEPSP response. Four $0.2-\mathrm{Hz}$ biphasic constant current pulses $(0.1 \mathrm{~ms} /$ polarity) were used for baseline recording and testing at each time point. Long-term potentiation (LTP) was elicited by "strong" tetanus (STET) consisting of three stimulus trains of 100 pulses at $100 \mathrm{~Hz}$ delivered at 10-min intervals (300 pulses in total). Data (fEPSP slope) were normalized to baseline and plotted as average \pm SEM. The average values of the slope function of the fEPSP (millivolts per milliseconds) for each time point were analyzed using paired $t$-test; $P<0.05$ was considered as statistically significant.

\section{Thioflavin S staining}

Thioflavin S staining was performed as previously described with the some modifications (Guntern et al, 1992; Sun et al, 2002). Briefly, mice were sacrificed and their brain quickly removed on ice. Left hemispheres were embedded in OCT (Tissue TEK) and stored at $-80^{\circ} \mathrm{C}$ until ready to section. Sagittal, $25-\mu \mathrm{m}$-thick sections were cut using a cryostat. And, five sets of five slides per brain containing four sections/slide were made by taking every $5^{\text {th }}$ section on the same slide, so that each set consisted of 20 representative sections throughout the brain. Sets to be used for thioflavin S staining were then fixed with $4 \%$ PFA for $25 \mathrm{~min}$ at room temperature, washed twice with $0.9 \% \mathrm{NaCl}$, and quickly rinsed with PBS. Staining was performed using filtered $0.05 \%$ thioflavin $\mathrm{S}$ in $50 \%$ ethanol for $8 \mathrm{~min}$ in the dark and differentiated with two changes of $80 \%$ ethanol for $10 \mathrm{~s}$. This was followed by three washes with large volumes of distilled water and an incubation step in high-concentrated phosphate buffer $(411 \mathrm{mM} \mathrm{NaCl}, 8.1 \mathrm{mM} \mathrm{KCl}, 30 \mathrm{mM}$ $\mathrm{Na}_{2} \mathrm{HPO}_{4}, 5.2 \mathrm{mM} \mathrm{KH} \mathrm{PO}_{4}, \mathrm{pH} 7.2$ ) at $4^{\circ} \mathrm{C}$ for $30 \mathrm{~min}$. Finally, slides were briefly rinsed in PBST and covered with coverslips using Vectashield Hard Set mounting media with DAPI (Vector). Slides were allowed to set in the dark at $4^{\circ} \mathrm{C}$ and imaged immediately thereafter. To control for background, unspecific staining, or tissue auto-fluorescence, slides from age-matched wild-type brains were used as negative controls. Images at $4 \times$ magnification of hippocampus and cortex were captured using an Olympus IX70 fluorescence microscope. Quantification of number of plaques, area covered by the plaque, and average plaque size was performed using the particle analysis tool of the ImageJ software. Statistical analysis was carried out using GraphPad Prism7.

\section{Biophysical experiments on $A \beta_{1-42}$}

Materials

$\mathrm{A} \beta_{1-42}$ was purchased from Bachem and Anaspec (Fremont, CA).

The phospholipids 1.2-dioleoyl-sn-glycero-3-phosphoserine (DOPS) and 1-palmitoyl-2-oleoyl-sn-glycero-3-phosphoethanolamine (POPE) were purchased from Avanti Polar Lipids (Alabaster, AL). All other chemicals were purchased from Sigma-Aldrich (St. Louis, $\mathrm{MO})$.

\section{Peptide handling}

For BLM experiments, $A \beta_{1-42}$ peptides were dissolved in Milli-Q water to a concentration of $1 \mathrm{mg} / \mathrm{ml}$ prior to being aliquoted for storage. These $50-\mu \mathrm{l}$ aliquots were stored at $-80^{\circ} \mathrm{C}$ for a maximum of 60 days before use. Samples were thawed only once. For AFM experiments, powder form of $A \beta_{1-42}$ (Bachem, Torrance, $C A$ ) was first dissolved in $1 \%$ ammonium hydroxide until the peptides were completely dissolved. They were subsequently sonicated for approximately $2 \mathrm{~min}$. Small volume of peptides were then aliquoted and lyophilized using a lyophilizer (Labconco FreeZone 2.5 Plus, Kansas City, KS). The aliquots were stored at $-80^{\circ} \mathrm{C}$ until used. For every experiment, aliquoted peptides were thawed and hydrated in $20 \mathrm{mM} \mathrm{NaOH}$ and HEPES buffer solutions at $\mathrm{pH} 7.4$, sequentially. The peptide concentration was measured using the $280 \mathrm{~nm} \mathrm{UV}$ absorbance $\left(\varepsilon=1,490 \mathrm{M}^{-1} \mathrm{~cm}^{-1}\right)$

\section{Peptide aggregation}

Synthetic $A \beta_{1-40}$ and $A \beta_{1-42}$ powders were dissolved in $20 \mathrm{mM}$ $\mathrm{NaOH}$ at $2 \mathrm{mg} / \mathrm{ml}$ concentration and incubated at $4^{\circ} \mathrm{C}$ for $1 \mathrm{~h}$ to dissolve their pre-existing aggregates. The $A \beta$ stock solutions were then brought to PBS, $\mathrm{pH} 7.4$, at a final peptide concentration of $0.4 \mathrm{mg} / \mathrm{ml}$, around $90 \mu \mathrm{M}$. After a short centrifugation $(16,000 \mathrm{~g}, 30 \mathrm{~min})$, the supernatants were used either directly, as the monomeric $\mathrm{A} \beta$, or following a 3 -h incubation at $37^{\circ} \mathrm{C}$ (without agitation), as the oligomeric-enriched $A \beta$ samples. The monomeric and oligomeric-enriched $A \beta$ samples were then added to primary neuronal cultures for membrane integrity and viability assays.

Electrical recording of planar lipid bilayer made of oxidized cholesterol/ n-decane

We prepared planar lipid bilayers using the Müller-Rudin technique (Benz et al, 1978). The membranes were formed from a $1 \%(\mathrm{w} / \mathrm{v})$ solution of oxidized cholesterol in $n$-decane. Oxidized cholesterol was prepared according to an established procedure (Benz et al, 1978). The lipid membranes were formed using a Teflon loop to spread the lipid solution across a hole with a surface area of approximately $0.3 \mathrm{~mm}^{2}$ in the wall dividing the two aqueous compartments in a Teflon cell. After the membrane had turned black, the $\mathrm{A} \beta_{1-42}$ peptide-containing protein fractions were added to the aqueous phase on one or both sides of the membrane at final concentrations 1 and $40 \mu \mathrm{M}$. For doping lipid bilayers with anle138b, the compound was dissolved in chloroform. The chloroform of a sample was then evaporated under vacuum. The lipid solution was added to the dry anle138b in such an amount that a final concentration of $1 \mathrm{mM}$ in the lipid solution was obtained. This lipid solution was used for the normal protocol of bilayer formation as described above. 
The current across the lipid bilayer membranes was measured with a pair of $\mathrm{Ag} / \mathrm{AgCl}$ electrodes with salt bridges switched in series with a voltage source and a highly sensitive current amplifier Keithley 427 (Keithley Instruments, INC. Cleveland, OH). The output signal of the amplifier was recorded by a strip chart recorder (Rikadenki Electronics GmbH, Freiburg, Germany). The temperature was kept at $20^{\circ} \mathrm{C}$ throughout the experiment.

\section{Planar lipid bilayer electrical recording using DOPS and POPE}

We prepared planar lipid bilayers using the so-called painted technique (Mueller et al, 1962). Anle138b was mixed with a 1:1 (w/w) solution of DOPS and POPE in chloroform at a concentration of $10 \mathrm{mM}$ with respect to the volume of the lipids. A lipid specific gravity of 0.8 was used for the calculation. This mixture was subsequently dried in a Rotavapor R-210 (Buchi) and resuspended in decane at a total lipid concentration of $20 \mathrm{mg} / \mathrm{ml}$ Bilayers with embedded anle138b were formed from this solution. Spontaneous membrane formation occurs following the addition of lipid directly over a pore with a diameter of $\sim 250 \mu \mathrm{m}$ in a Delrin septum (Warner Instruments, Delrin perfusion cup, volume $1 \mathrm{ml}$ ). In previous studies, this membrane composition was shown to be stable for long recording times $(\sim 4 \mathrm{~h}$; Capone et al, 2012). Control experiments establishing the stability of membranes formed with the addition of anle138b were performed. We used $150 \mathrm{mM} \mathrm{KCl}, 10 \mathrm{mM}$ HEPES (pH 7.4), and $1 \mathrm{mM} \mathrm{MgCl}_{2}$ as the electrolyte.

We observed the following difficulty in the preparation of anle138b loaded lipids. Anle138b was dissolved in decane alon with the lipids prior to membrane painting. Since anle138b is soluble in both the decane and the lipids, the distribution of compound in the lipid membrane that spontaneously forms upon lipid deposition over the aperture can vary. Lipid monolayers bind to either side of the partition and the bilayer membrane forms as the monolayers fuse together at the center, excluding the decane solvent to the perimeter. This solvent annulus acts as a bridge to the Delrin partition and is essential for membrane stability (White, 1972). If a significant proportion of the anle138b is mobile in the decane, the compound could be partitioned to the solvent annulus rather than incorporated into the membrane leading to BLM results that appear similar to that seen with $A \beta_{1-42}$ in the absence of compound. This can explain why anle138b modulated the activity of the pores in only $50 \%$ of the cases.

Before performing electrical recordings, we verified that the bilayer was stable for several minutes with low conductance $(<10 \mathrm{pS})$ across $\pm 100 \mathrm{mV}$ applied voltage and that the system capacitance was $>110 \mathrm{pF}$. When both criteria were met, peptide was added directly to the cis (hot wire) side and stirred for $5 \mathrm{~min}$. Peptide concentration in the bilayer chamber was approximately $10 \mu \mathrm{M}$. Bilayer stability was monitored by periodic capacitance measurements throughout the course of the experiment.

All traces were recorded in voltage clamp mode using the $2 \mathrm{kHz}$ built-in filter cutoff of our BC-535 amplifier (Warner Instruments, Hamden, CT). A sampling frequency of $15 \mathrm{kHz}$ was used for all data acquisition. We used a custom-made LabVIEW program to record the current and Clampfit 10.2 (Molecular Devices, Sunnyvale, CA) to analyze traces. We have filtered the recorded current versus time traces with a digital Gaussian low-pass filter and a cutoff frequency of $50 \mathrm{~Hz}$ for representation in figures.

\section{Lipid bilayer preparation for AFM imaging}

For liposome preparation, DOPS and POPE lipids were used in a 1:1 ratio (Avanti Polar Lipids, Alabaster, Al). Liposomes were prepared by mixing $20 \mu \mathrm{l}$ of each lipid ( $5 \mathrm{mg} / \mathrm{ml}$ ) dissolved in chloroform, and anle138b, also in chloroform, was added to a 1,000:1 lipid to anle138b molar ratio. Then, liposomes were allowed to dry overnight in vacuum. The dried lipid film (and anle138b) was hydrated with peptide solution (1:60 peptide to lipid molar ratio) to facilitate peptide incorporation in the lipid bilayer, resulting in proteoliposome formation. For controls, the dried lipid film (and anle138b) was hydrated with $200 \mu \mathrm{l}$ of HEPES buffer and vortexed occasionally for an hour. The large multilamellar vesicles formed with this procedure were sonicated for $5 \mathrm{~min}$. Supported lipid bilayers were formed by (proteo)liposome rupture and fusion on the mica substrate (Lin et al, 2001; Quist et al, 2005; Liu et al, 2006; Umehara et al, 2010; Connelly et al, 2012a,b). Lipid concentrations of $0.1-1 \mathrm{mg} / \mathrm{ml}$ were deposited on freshly cleaved mica and incubated for $\sim 10 \mathrm{~min}$ on a hot plate above the lipid transition temperature to facilitate fusion of the ruptured proteoliposomes on the mica surface. As a last step, samples were rinsed with buffer to remove unruptured proteoliposomes still in solution.

\section{AFM imaging on membranes}

Topographic images were acquired using a Multimode AFM equipped with a Nanoscope V controller (Bruker, Santa Barbara, CA). Silicon nitride cantilevers with nominal spring constants of $0.08 \mathrm{~N} / \mathrm{m}$ (OMCL-TR400, Olympus) were employed for imaging in fluid using the tapping mode. Resonance frequencies of $\sim 8 \mathrm{kHz}$ and drive amplitudes under $100 \mathrm{mV}$ were used. All experiments were performed at room temperature. Actual spring constants were measured to be within $10 \%$ of its nominal value using thermal tune before the experiments. To measure outer pore diameters, tip broadening was taken into account by modeling the inserted oligomers as a spherical cap protruding a height $\mathrm{h}$ above the surface of the lipid bilayer in contact with a spherical tip of radius $\mathrm{R}$ (Lee et al, 2014).

\section{Cell membrane integrity and viability}

\section{CYQUANT}

Primary neuronal cultures were produced from E17.5 CD1 Swiss embryos. On DIV, 10 cultures were treated conditioned medium supplemented with anle138b to a final concentration of $1 \mu \mathrm{M}$ in $0.05 \%$ DMSO (Roth, A994.2) or 0.05\% DMSO as vehicle. After $24 \mathrm{~h}$ $\mathrm{A} \beta_{1-40}$ oligomers, monomers or buffer ( $n=4$ each) was applied at $10 \mu \mathrm{M}$ and incubated for $48 \mathrm{~h}$. CyQUANT ${ }^{\circledR}$ Direct Cell Proliferation Assay (Thermo Fisher, C35011) was used according to manufacturer's protocol to determine membrane integrity. After 30-min incubation, fluorescence was measured with a Tecan infinite 200. Statistical analysis was performed in GraphPad Prism.

\section{MTT assay}

Cell viability was measured using the MTT assay with the same sample preparation as for the CyQUANT assay. Briefly, after anle138b and $A \beta_{1-40}$ treatment, the cell culture medium was supplemented with MTT to a final concentration of $0.5 \mathrm{mg} / \mathrm{ml}$ and incubated for $1 \mathrm{~h}$ at $37^{\circ} \mathrm{C}$ in a standard cell culture incubator. Subsequently, medium was removed and metabolites suspended in $500 \mu \mathrm{l}$ DMSO. Absorption at $800 \mathrm{~nm}$ was measured using a Tecan infinite 200. 


\section{Transgenic flies and survival assays}

$\mathrm{W}\left[{ }^{1118}\right] ; \mathrm{P}\{\mathrm{UAS}-$ Arctic A 1 1-42 (Glu22Gly) $\}$ (arc2E) transgenic flies were kindly provided by D. Crowther (Cambridge, UK; Crowther et al, 2005). The co-expression of Gal4 under a tissue specific promotor leads to activation of an upstream activating sequence (UAS) element that activates the transcription of A $\beta 42$ upon Gal4-UAS binding (Brand \& Perrimon, 1993). We controlled the temporal expression of the Gal4-UAS system by a temperature-sensitive Gal $80^{\text {ts }}$ under a ubiquitously expressing tubulin promoter (tubGal $80^{\text {ts }}$ ) (McGuire et al, 2003). Gal80 ${ }^{\text {ts }}$ represses the transcriptional activity of Gal4 at the permissive temperature $\left(18^{\circ} \mathrm{C}\right)$. Upon a shif to the non-permissive temperature $\left(30^{\circ} \mathrm{C}\right), \mathrm{Gal} 80^{\text {ts }}$ becomes dysfunctional and releases the Gal4-UAS system to allow transgene expression. Neuron-specific and temperature-inducible expression of $\mathrm{A} \beta 42$ was achieved by crossing the $\left.\mathrm{w}^{1118}\right]$; Gal4-elav/CyO; TM2/TM6 and w $\left[{ }^{1118}\right]$; Sp/CyO; tubGal80 $0^{\text {ts }} / \mathrm{TM} 2$ lines obtained from Bloomington (Bloomington Drosophila Stock Center (BDSC), Indiana University, USA) to generate $\mathrm{w}\left[{ }^{1118}\right]$; Gal4-elav/(CyO); tubGal80 $0^{\text {ts }} /$ (TM6). The $\left.\mathrm{w}^{11118}\right]$; P $\{$ UAS-Arctic A $\beta$ 1-42 (Glu22Gly) $\}$ line was crossed with $\left.\mathrm{w}^{1118}\right]$; Gal4-elav/(CyO);tubGal80 ${ }^{\text {ts }} /(\mathrm{TM} 6)$. $\mathrm{w}\left[{ }^{1118}\right]$; Gal4-elav/UAS-arc2E;tubGal80/+ were used for subsequent survival assays. As an overexpression control, we crossed $\mathrm{w}\left[{ }^{1118}\right]$; Gal4-elav/(CyO);tubGal80/(TM6) with $\quad \mathrm{w}\left[{ }^{1118}\right] ; \quad \mathrm{P}\{\mathrm{w}[+\mathrm{mC}]=\mathrm{UAS}-$ lacZ.NZ 20 b (Bloomington Drosophila Stock Center (BDSC), Indiana University, USA) to obtain $\mathrm{w}\left[{ }^{1118}\right]$; Gal4-elav/+; tubGal $80^{\mathrm{ts}}$ / UAS-lacZ.

\section{Survival assay}

Male flies expressing $A \beta 42$ Glu22Gly arctic mutation and control flies expressing lac $Z$ were collected and maintained under a 12 -h light-dark cycle in $60 \%$ relative humidity and equal population density per vial. Within $24 \mathrm{~h}$ after eclosion from the pupae, all flies were transferred to the restrictive temperature. Compounds were dissolved at $10 \mathrm{mM}$ in DMSO and mixed with yeast. Fresh food was added every $2^{\text {nd }}-3^{\text {rd }}$ day. As a control, A $\beta 42$ Glu22Gly transgenic and lacZ control flies were treated with DMSO food without addition of compounds. Surviving flies were counted every $2^{\text {nd }}-3^{\text {rd }}$ day. The resulting Kaplan-Meier survival curves were calculated using SSPS software. Differences between the genotypes were assessed by logrank test (SSPS software).

\section{Statistical analysis}

Unless specifically mentioned otherwise, data were analyzed by unpaired Student's $t$-test, two tailed $t$-test, Bonferroni test for multiple comparisons, or one and two-way and ANOVA (analysis of variance) when appropriate. Errors are displayed as standard error of mean (SEM). Unless otherwise stated, analysis was performed using GraphPad Prism.

\section{Data availability}

RNA-sequencing datasets are available via GEO accession number: GSE104424.

Expanded View for this article is available online.

\section{The paper explained}

\section{Problem}

A hallmark of Alzheimer's disease is the aggregation of $A \beta$, yet the mechanism of dysfunction of neurons and neuronal death is unclear and disease-modifying therapeutic intervention is not yet possible.

\section{Results}

Using the APPPS1 overexpression model in mice, we show that the diphenylpyrazole compound anle138b, an orally available small molecule reaching $30 \mu \mathrm{M}$ concentration in the brain when given orally, ameliorated disease phenotypes such as hippocampal LTP, spatial reference memory, and transcriptional homeostasis. Biophysical analysis suggests that pore formation contributes to the dysfunctionalization and death of neurons, while anle138b prevents pore formation and thereby rescues these effects.

Impact

Our work provides evidence of the molecular mechanisms by which $A \beta$ aggregation is contributing to Alzheimer's disease, namely pore $A \beta$ aggregation is contributing to Alzheimer's disease, namely pore
formation in membranes by $A \beta$ oligomers whose conductivity is reduced by more than a factor of 10 by anle138b treatment. The pore conductivity reduction correlates with the full refunctionalization as measured by LTP when anle138b is given before or after the onset of amyloid deposition. Also memory function is improved. Our data suggest that therapeutical effects can be expected to be achieved in AD patients with anle138b.

\section{Acknowledgements}

We thank Lina-Maria Jaime Tobonand Inga Urban for assistance. This work was supported by core funds from the DZNE (to AF, MZ, and MF), the Max Planck Society (to GE and CG), the Hans and Ilse Breuer Award for Alzheimer's disease (to AF), the DFC project F1981/9-1 (to AF), the EU (ERC consolidator grant to AF), and the DFC (SFB803 project A04 to CG). HU holds a PhD fellowship from the Hans and Ilse Breuer Foundation. MF is supported by the CoEN Initiative CoEN3018 and the SFB1089 C01. NV is supported by the Malta Council for Science \& Technology through the National Research \& Innovation Programme (R\&l-2008-068) and the University of Malta (PHBR06). AC was supported by the Malta Government Scholarship Scheme. J.L., A.L.G., and R.L. acknowledge support from the National Institute on Aging of National Institutes of Health (Grant AG028709).

\section{Author contributions}

AHM: coordinated the experiments, performed behavior testing of mice, RNAseq, and amyloid plaque analysis. HU: performed all experiments related to primary hippocampal neurons. ALG: performed the electrophysiology with black membranes. JL: did AFM measurements of A $\beta$ on membranes. SR: provided anle138b. HYA: performed behavior experiments. C): analyzed RNAseq data. EB: performed pathway analysis. GG: performed the LTP measurements. AL: provided anle138b. NR-G: generated synthetic $A \beta$ used for the CyQuant and MTT assay. FTA: did the AFM measurements. PW: performed fly experiments. AS: designed and analyzed fly experiments. MZ: generated synthetic $A \beta$, wrote the manuscript. RB: performed the BLM measurement. AG: discussed the data. MK: conceived and supervised experiments, discussed and analyzed specifically the electrophysiological experiments, wrote the manuscript. RL: conceived and supervised experiments, specifically supervised the AFM measurements and interpreted them, wrote the manuscript. CG: conceived and supervised experiments, wrote the manuscript. GE: conceived and supervised experiments, wrote the manuscript. AF: conceived and supervised experiments, wrote the manuscript. LK: helped to perform and analyze 
RNA-seq data. JW and MF treated Tau mice with anle138b and collected tissue. MC, AC, and NV performed and interpreted the experiments on cytochrome $\mathrm{C}$ release from mitochondria.

\section{Conflict of interest}

AG and CG are co-founders of MODAG. AL is partly employed by MODAC.

\section{References}

Arce FT, Jang H, Ramachandran S, Landon PB, Nussinov R, Lal R (2011) Polymorphism of amyloid $\beta$ peptide in different environments: implications for membrane insertion and pore formation. Soft Matter 7 : $5267-5273$

Arispe N, Rojas E, Pollard HB (1993) Alzheimer disease amyloid beta protein forms calcium channels in bilayer membranes: blockade by tromethamine and aluminum. Proc Natl Acad Sci USA 90: 567-571

Benito E, Urbanke H, Ramachandran B, Barth J, Halder R, Awasthi A, Jain C, Capece V, Burkhardt S, Navarro-Sala M et al (2015) HDAC inhibitordependent transcriptome and memory reinstatement in cognitive decline models. J Clin Invest 17: 3572-3584

Benz R, Janko K, Boos W, Lauger P (1978) Formation of large, ion-permeable membrane channels by matrix protein (Porin) of Escherichia-Coli. Biochem Biophys Acta 511: 305-319

Bieschke J, Russ J, Friedrich RP, Ehrnhoefer DE, Wobst H, Neugebauer K, Wanker EE (2010) EGCG remodels mature alpha-synuclein and amyloidbeta fibrils and reduces cellular toxicity. Proc Natl Acad Sci USA 107: $7710-7715$

Brand AH, Perrimon N (1993) Targeted gene expression as a means of altering cell fates and generating dominant phenotypes. Development 2 401-415

Capone R, Jang H, Kotler SA, Kagan BL, Nussinov R, Lal R (2012) Probing structural features of Alzheimer's amyloid-beta pores in bilayers using site-specific amino acid substitutions. Biochemistry 51: 776-785

Connelly L, Arce FT, Jang H, Capone R, Kotler SA, Ramachandran S, Kagan BL, Nussinov R, Lal R (2012a) Atomic force microscopy and MD simulations reveal pore-like structures of all-D-Enantiomer of Alzheimer's $\beta$-amyloid peptide: relevance to the ion channel mechanism of AD pathology. J Phys Chem B 116: $1728-1735$

Connelly L, Jang H, Arce FT, Ramachandran S, Kagan BL, Nussinov R, Lal R (2012b) Effects of point substitutions on the structure of toxic Alzheimer's $\beta$-amyloid channels: atomic force microscopy and molecular dynamics simulations. Biochemistry 51: $3031-3038$

Crowther DC, Kinghorn KJ, Miranda E, Page R, Curry JA, Duthie FA (2005) Intraneuronal Abeta, non-amyloid aggregates and neurodegeneration in a Drosophila model of Alzheimer's disease. Neuroscience 132: 123-135

Demuro A, Smith M, Parker I (2011) Single-channel $\mathrm{Ca}(2+)$ imaging implicates Aß1-42 amyloid pores in Alzheimer's disease pathology. J Cell Biol 195 515-524

Diaz JC, Simakova O, Jacobson KA, Arispe N, Pollard HB (2009) Small molecule blockers of the Alzheimer $A$ beta calcium channel potently protect neurons from A beta cytotoxicity. Proc Natl Acad Sci USA 106: $3348-3353$

Djebali S, Davis CA, Merkel A, Dobin A, Lassmann T, Mortazavi A, Tanzer A Lagarde J, Lin WSF, Xue C et al (2012) Landscape of transcription in human cells. Nature 489: 101-108

Doig AJ, Derreumaux P (2015) Inhibition of protein aggregation and amyloid formation by small molecules. Curr Opin Struct Biol 30: 50-56
Ehrnhoefer DE, Bieschke J, Boeddrich A, Herbst M, Masino L, Lurz R, Engemann S, Pastore A, Wanker EE (2008) EGCG redirects amyloidogenic polypeptides into unstructured, off-pathway oligomers. Nat Struct Mol Biol 15: $558-566$

Fischer A (2014a) Epigenetic memory: the Lamarckian brain. EMBO / 33 945-967

Fischer A (2014b) Targeting histone-modifications in Alzheimer's disease. What is the evidence that this is a promising therapeutic avenue? Neuropsychopharmacology 80: 95-102

Fischer A (2016) Environmental enrichment as a method to improve cognitive function. What can we learn from animal models? Neurolmage 1: 42-47

Gillman AL, Jang H, Lee J, Ramachandran S, Kagan BL, Nussinov R, Arce FT (2014) Activity and architecture of pyroglutamate modified amyloid- $\beta$ (AßpE3-42) pores. J Phys Chem B 118: $7335-7344$

Goate A, Hardy J (2012) Twenty years of Alzheimer's disease-causing mutations. J Neurochem 120: $3-8$

Guntern R, Bouras C, Hof PR, Vallet PG (1992) An improved thioflavine-s method for staining neurofibrillary tangles and senile plaques in Alzheimers disease. Experientia 48: 8-10

Haass C, Selkoe DJ (2007) Soluble protein oligomers in neurodegeneration: lessons from the Alzheimer's amyloid beta-peptide. Nat Reu Mol Cell Biol 8: $112-116$

Iversen LL, Mortishire-Smith RJ, Pollack SJ, Shearman MS (1995) The toxicity in vitro of beta-amyloid protein. Biochem J 311: 1-16

Jakob-Roetne R, Jacobsen H (2009) Alzheimer's disease: from pathology to therapeutic approaches. Angew Chem Int Ed 48: 3030-3059

Jang H, Zheng J, Lal R, Nussinov R (2008) New structures help the modeling of toxic amyloid $\beta$ ion channels. Trends Biochem Sci 33: $91-100$

Jankowsky JL, Slunt HH, Ratovitski T, Jenkins NA, Copeland NG, Borchelt DR (2001) Co-expression of multiple transgenes in mouse CNS: a comparison of strategies. Biomol Eng 17: 157-165

Jankowsky JL, Fadale DJ, Anderson J, Xu GM, Gonzales V, Jenkins NA, Copeland NG, Lee MK, Younkin LH, Wagner SL et al (2004) Mutant presenilins specifically elevate the levels of the 42 residue beta-amyloid peptide in vivo: evidence for augmentation of a 42 -specific gamma secretase. Hum Mol Genet 13: 159-170

Kummer MP, Hammerschmidt T, Martinez A, Terwel D, Eichele C, Witten A, Figura S, Stoll M, Schwartz S, Pape HC et al (2014) Ear2 deletion causes early memory and learning deficits in APP/PS1 mice. J Neurosci 34: $8845-8854$

Lalonde R, Kim HD, Maxwell JA, Fukuchi K (2005) Exploratory activity and spatial learning in 12-month-old APP(695)SWE/co+PS1/Delta E9 mice with amyloid plaques. Neurosci Lett 390: 87-92

Lee J, Gillman AL, Jang H, Ramachandran S, Kagan BL, Nussinov R, Arce FT (2014) Role of the fast kinetics of pyroglutamate-modified amyloid- $\beta$ oligomers in membrane binding and membrane permeability. Biochemistry 53: $4704-4714$

Lin HAl, Bhatia R, Lal R (2001) Amyloid $\beta$ protein forms ion channels: implications for Alzheimer's disease pathophysiology. FASEB J 15: $2433-2444$

Liu F, Arce FT, Ramachandran S, Lal R (2006) Nanomechanics of hemichannel conformations - Connexin flexibility underlying channel opening and closing. I Biol Chem 281: 23207-23217

Liu M, Chen F, Sha L, Wang S, Tao L, Yao L, He M, Yao Z, Liu H, Zhu Z et al (2014) (-)-Epigallocatechin-3-gallate ameliorates learning and memory deficits by adjusting the balance of TrkA/p75NTR signaling in APP/PS1 transgenic mice. Mol Neurobiol 49: 1350-1363 
Love MI, Huber W, Anders S (2014) Moderated estimation of fold change and dispersion for RNA-seq data with DESeq2. Genome Biol 15: 550 Matarin M, Salih DA, Yasvoina M, Cummings DM, Guelfi S, Liu W, Nahaboo Solim MA, Moens TG, Paublete RM, Ali SS et al (2015) A genome-wide gene-expression analysis and database in transgenic mice during development of amyloid or tau pathology. Cell Rep 10: 633-644

McGuire SE, Le PT, Osborn AJ, Matsumoto K, Davis RL (2003) Spatiotemporal rescue of memory dysfunction in Drosophila. Science 302: 1765-1768

Morris R (1984) Developments of a water-maze procedure for studying spatial learning in the rat. J Neurosci Methods 11: 47-60

Mueller P, Rudin DO, Tien HT, Wescott WC (1962) Reconstitution of cell membrane structure in vitro and its transformation into an excitable system. Nature 194: 979-980

Querfurth HW, LaFerla FM (2010) Alzheimer's disease. N Engl J Med 362 $329-344$

Quist A, Doudevski I, Lin H, Azimova R, Ng D, Frangione B, Kagan B, Chiso J, Lal R (2005) Amyloid ion channels: a common structural link for proteinmisfolding disease. Proc Natl Acad Sci USA 102: 10427-10432

Reiserer RS, Harrison FE, Syverud DC, McDonald MP (2007) Impaired spatial learning in the APP(Swe)+PSEN1 Delta E9 bigenic mouse model of Alzheimer's disease. Genes Brain Behav 6: 54-65

Sarkar B, Mithu VS, Chandra B, Mandal A, Chandrakesan M, Bhowmik D Madhu PK, Maiti S (2014) Significant structural differences between transient amyloid-beta oligomers and less-toxic fibrils in regions known to harbor familial Alzheimer's mutations. Angew Chem Int Ed 53 $6888-6892$

Selkoe DJ, Hardy J (2016) The amyloid hypothesis of Alzheimer's disease at 25 years. EMBO Mol Med 8: 595-608

Sevigny J, Chiao P, Bussière T, Weinreb PH, Williams L, Maier M, Dunstan R, Salloway S, Chen T, Ling Y et al (2016) The antibody aducanumab reduces A $\beta$ plaques in Alzheimer's disease. Nature 537: $50-56$

Shankar GM, Bloodgood BL, Townsend M, Walsh DM, Selkoe DJ, Sabatini BL (2007) Natural oligomers of the Alzheimer amyloid-beta protein induce reversible synapse loss by modulating an NMDA-type glutamate receptordependent signaling pathway. J Neurosci 27: $2866-2875$
Spires TL, Hyman BT (2004) Neuronal structure is altered by amyloid plaques. Reu Neurosci 15: 267-278

Spruijt CG, Gnerlich F, Smits AH, Pfaffeneder T, Jansen PW, Bauer C, Münzel M, Wagner M, Müller M, Khan F et al (2013) Dynamic readers for 5(hydroxy)methylcytosine and its oxidized derivatives. Cell 152: 1146-1159

Sun A, Nguyen XV, Bing G (2002) Comparative analysis of an improved thioflavin-s stain, Gallyas silver stain, and immunohistochemistry for neurofibrillary tangle demonstration on the same sections. J Histochem Cytochem 50: $463-472$

Tanzi RE (2005) The synaptic Abeta hypothesis of Alzheimer disease. Nat Neurosci 8: $977-979$

Umehara T, Nakamura Y, Jang MK, Nakano K, Tanaka A, Ozato K, Padmanabhan B, Yokoyama S (2010) Structural basis for acetylated histone $\mathrm{H} 4$ recognition by the human BRD2 bromodomain. J Biol Chem 285: $7610-7618$

Vassar R, Kuhn PH, Haass C, Kennedy ME, Rajendran L, Wong PC, Lichtenthaler SF (2014) Function, therapeutic potential and cell biology of BACE proteases: current status and future prospects. J Neurochem 130: 4-28

Wagner J, Ryazanov S, Leonov A, Levin J, Shi S, Schmidt F, Prix C, PanMontojo F, Bertsch U, Mitteregger-Kretzschmar G et al (2013) Anle138b: a novel oligomer modulator for disease-modifying therapy of neurodegenerative diseases such as prion and Parkinson's disease. Acta Neuropathol 125: 795-813

Wagner J, Krauss S, Shi S, Ryazanov S, Steffen J, Miklitz C, Leonov A, Kleinknecht A, Göricke B, Weishaupt JH et al (2015) Reducing tau aggregates with anle138b delays disease progression in a mouse model of tauopathies. Acta Neuropathol 130: 619-631

White SH (1972) Analysis of the torus surrounding planar lipid bilayer membranes. Biophys J 12: $432-445$

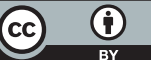

License: This is an open access article under the terms of the Creative Commons Attribution 4.0 License, which permits use, distribution and reproduction in any medium, provided the original work is properly cited. 


\title{
Co-Author release forms
}

For the publication entitled "The diphenylpyrazole compound anle138b blocks Ab channels and rescues disease phenotypes in a mouse model for amyloid pathology".

\author{
$16 / 11 / 2017$ \\ Hendrik Urbanke has my permission to include material, which was accepted for \\ publication, of which I was a co-author, in his doctoral dissertation. \\ - Ana Martinez Hernandez, Hendrik Urbanke, Alan L. Gillman, Joon Lee, Sergey \\ Ryazanov, Hope Y. Agbemenyah, Eva Benito, Gaurav Jain, Lalit Kaurani, \\ Gayane Grigorian, Andrei Leonov, Nasrollah Rezai-Ghaleh, Petra Wilken \\ Fernando Teran Arce, Markus Zweckstetter, Roland Benz, Armin Giese, Anja \\ Schreider, Martin Korte*, Ratnesh Lal ${ }^{*}$, Christian Griesinger*, Gregor Eichele* \\ Andire Fischer* "The diphenylpyrazol compound anle138b blocks A $\beta$ channels \\ and scues disease phenotypes in a mouse model for amyloid pathology"

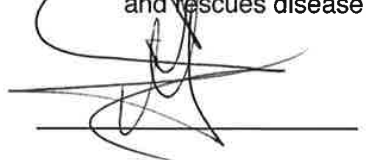

Ana Martinez Hernandez

$16 / 11 / 2017$

Hendrik Urbanke has my permission to include material, which was accepted for publication, of which I was a co-author, in his doctoral dissertation.

- Ana Martinez Hernandez, Hendrik Urbanke, Alan L. Gillman, Joon Lee, Sergey Ryazanov, Hope Y. Agbemenyah, Eva Benito, Gaurav Jain, Lalit Kaurani, Gayane Grigorian, Andrei Leonov, Nasrollah Rezai-Ghaleh, Petra Wilken, Fernando Teran Arce, Markus Zweckstetter, Roland Benz, Armin Giese, Anja Schneider, Martin Korte*, Ratnesh Lal*, Christian Griesinger*, Gregor Eichele*, Andre Fischer* "The diphenylpyrazol compound anle138b blocks $A \beta$ channels and rescues disease phenotypes in a mouse model for amyloid pathology"

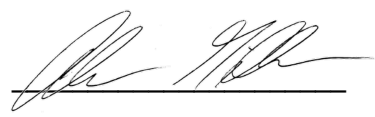

Alan L. Gillman

$16 / 11 / 2017$

Hendrik Urbanke has my permission to include material, which was accepted for publication, of which I was a co-author, in his doctoral dissertation.

- Ana Martinez Hernandez, Hendrik Urbanke, Alan L. Gillman, Joon Lee, Sergey Ryazanov, Hope Y. Agbemenyah, Eva Benito, Gaurav Jain, Lalit Kaurani, Gayane Grigorian, Andrei Leonov, Nasrollah Rezai-Ghaleh, Petra Wilken, Fernando Teran Arce, Markus Zweckstetter, Roland Benz, Armin Giese, Anja Schneider, Martin Korte*, Ratnesh Lal ${ }^{*}$, Christian Griesinger ${ }^{*}$, Gregor Eichele*, Andre Fischer" "The diphenylpyrazol compound anle138b blocks $A \beta$ channels and rescues disease phenotypes in a mouse model for amyloid pathology"

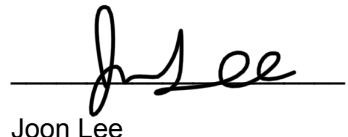




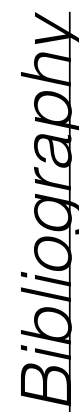




\section{Bibliography}

(2014/15). "World Dementia Council, Annual Report."

Abramowicz, M. (2016). "Microcephaly risk with RUSC2." Dev Med Child Neurol 58(12): 1211-1212.

Agbandje-McKenna, M. and J. Kleinschmidt (2011). AAV Capsid Structure and Cell Interactions. Adeno-Associated Virus: Methods and Protocols. R. O. Snyder and P. Moullier. Totowa, NJ, Humana Press: 47-92.

Agranoff, B. W. and P. D. Klinger (1964). "PUROMYCIN EFFECT ON MEMORY FIXATION IN THE GOLDFISH." Science 146(3646): 952-953.

Ahmed, T., A. Van der Jeugd, D. Blum, M. C. Galas, R. D'Hooge, L. Buee and D. Balschun (2014). "Cognition and hippocampal synaptic plasticity in mice with a homozygous tau deletion." Neurobiol Aging 35(11): 2474-2478.

Alarcon, J. M., G. Malleret, K. Touzani, S. Vronskaya, S. Ishii, E. R. Kandel and A. Barco (2004). "Chromatin acetylation, memory, and LTP are impaired in CBP+/- mice: a model for the cognitive deficit in Rubinstein-Taybi syndrome and its amelioration." Neuron 42(6): 947-959.

Allis, C. D., T. Jenuwein and D. Reinberg (2007). "Epigenetics. Cold Spring Harbor Laboratory Press. 2007." Genetical Research 89(2): 124-125.

Alonso, A. C., T. Zaidi, I. Grundke-lqbal and K. Iqbal (1994). "Role of abnormally phosphorylated tau in the breakdown of microtubules in Alzheimer disease." Proc Natl Acad Sci U S A 91(12): 5562-5566.

Alwadei, A. H., R. Benini, A. Mahmoud, A. Alasmari, E. J. Kamsteeg and M. Alfadhel (2016). "Loss-of-function mutation in RUSC2 causes intellectual disability and secondary microcephaly." Dev Med Child Neurol 58(12): 1317-1322.

Amenta, A. R., H. E. Creely, M. L. Mercado, H. Hagiwara, B. A. McKechnie, B. E. Lechner, S. G. Rossi, Q. Wang, R. T. Owens, E. Marrero, L. Mei, W. Hoch, M. F. Young, D. J. McQuillan, R. L. Rotundo and J. R. Fallon (2012). "Biglycan is an extracellular MuSK binding protein important for synapse stability." J Neurosci 32(7): 2324-2334.

Anders, S., A. Reyes and W. Huber (2012). "Detecting differential usage of exons from RNA-seq data." Genome Res 22(10): 2008-2017.

Anders, S., A. Reyes and W. Huber (2012). "Detecting differential usage of exons from RNA-seq data." Genome Research 22(10): 2008-2017.

Arispe, N., E. Rojas and H. B. Pollard (1993). "Alzheimer disease amyloid beta protein forms calcium channels in bilayer membranes: blockade by tromethamine and aluminum." Proc Natl Acad Sci U S A 90(2): 567-571.

Arrigo, A. P., J. L. Darlix and P. F. Spahr (1983). "A cellular protein phosphorylated by the avian sarcoma virus transforming gene product is associated with ribonucleoprotein particles." Embo j 2(3): 309-315.

Attar, A., T. Liu, W. T. Chan, J. Hayes, M. Nejad, K. Lei and G. Bitan (2013). "A shortened Barnes maze protocol reveals memory deficits at 4-months of age in the tripletransgenic mouse model of Alzheimer's disease." PLoS One 8(11): e80355. 
Aukrust, I., H. Hollas, E. Strand, L. Evensen, G. Trave, T. Flatmark and A. Vedeler (2007). "The mRNA-binding site of annexin A2 resides in helices C-D of its domain IV." J Mol Biol 368(5): 1367-1378.

Aukrust, I., L. A. Rosenberg, M. M. Ankerud, V. Bertelsen, H. Hollas, J. Saraste, A. K. Grindheim and A. Vedeler (2017). "Post-translational modifications of Annexin A2 are linked to its association with perinuclear nonpolysomal mRNP complexes." FEBS Open Bio 7(2): 160-173.

Azevedo, F. A., L. R. Carvalho, L. T. Grinberg, J. M. Farfel, R. E. Ferretti, R. E. Leite, W. Jacob Filho, R. Lent and S. Herculano-Houzel (2009). "Equal numbers of neuronal and nonneuronal cells make the human brain an isometrically scaled-up primate brain." J Comp Neurol 513(5): 532-541.

Bach, D. R., A. Tzovara and J. Vunder (2017). "Blocking human fear memory with the matrix metalloproteinase inhibitor doxycycline." Mol Psychiatry.

Bacskai, B. J., B. Hochner, M. Mahaut-Smith, S. R. Adams, B. K. Kaang, E. R. Kandel and R. Y. Tsien (1993). "Spatially resolved dynamics of cAMP and protein kinase A subunits in Aplysia sensory neurons." Science 260(5105): 222-226.

Bailey, C. H., D. Bartsch and E. R. Kandel (1996). "Toward a molecular definition of long-term memory storage." Proc Natl Acad Sci U S A 93(24): 13445-13452.

Bailey, C. H. and E. R. Kandel (2008). "Synaptic remodeling, synaptic growth and the storage of long-term memory in Aplysia." Prog Brain Res 169: 179-198.

Bannister, A. J. and T. Kouzarides (2011). "Regulation of chromatin by histone modifications." Cell Res 21(3): 381-395.

Barco, A., J. M. Alarcon and E. R. Kandel (2002). "Expression of constitutively active CREB protein facilitates the late phase of long-term potentiation by enhancing synaptic capture." Cell 108(5): 689-703.

Barnes, C. A. (1979). "Memory deficits associated with senescence: a neurophysiological and behavioral study in the rat." $\mathrm{J}$ Comp Physiol Psychol 93(1): 74-104.

Barondes, S. H. and H. D. Cohen (1966). "Puromycin effect on successive phases of memory storage." Science 151(3710): 594-595.

Bartholomeeusen, K., Y. Xiang, K. Fujinaga and B. M. Peterlin (2012). "Bromodomain and extraterminal (BET) bromodomain inhibition activate transcription via transient release of positive transcription elongation factor $b$ (P-TEFb) from 7SK small nuclear ribonucleoprotein." J Biol Chem 287(43): 36609-36616.

Bast, T., W. N. Zhang and J. Feldon (2001). "The ventral hippocampus and fear conditioning in rats. Different anterograde amnesias of fear after tetrodotoxin inactivation and infusion of the GABA(A) agonist muscimol." Exp Brain Res 139(1): 39-52.

Bender, A. R., M. Naveh-Benjamin and N. Raz (2010). "Associative Deficit in Recognition Memory in a Lifespan Sample of Healthy Adults." Psychology and aging 25(4): 940-948.

Benito, E., B. Ramachandran, H. Schroeder, G. Schmidt, H. Urbanke, S. Burkhardt, V. Capece, C. Dean and A. Fischer (2017). "The BET/BRD inhibitor JQ1 improves brain plasticity in WT and APP mice." Translational Psychiatry 7(9): e1239.

Benito, E., H. Urbanke, B. Ramachandran, J. Barth, R. Halder, A. Awasthi, G. Jain, V. Capece, S. Burkhardt, M. Navarro-Sala, S. Nagarajan, A. L. Schutz, S. A. Johnsen, S. Bonn, R. Luhrmann, C. Dean and A. Fischer (2015). "HDAC inhibitor-dependent 
transcriptome and memory reinstatement in cognitive decline models." J Clin Invest 125(9): 3572-3584.

Bharadwaj, A., M. Bydoun, R. Holloway and D. Waisman (2013). "Annexin A2 heterotetramer: structure and function." Int J Mol Sci 14(3): 6259-6305.

Biernat, J., N. Gustke, G. Drewes, E. M. Mandelkow and E. Mandelkow (1993). "Phosphorylation of Ser262 strongly reduces binding of tau to microtubules: distinction between PHF-like immunoreactivity and microtubule binding." Neuron 11.

Bindea, G., B. Mlecnik, H. Hackl, P. Charoentong, M. Tosolini, A. Kirilovsky, W. H. Fridman, F. Pages, Z. Trajanoski and J. Galon (2009). "ClueGO: a Cytoscape plug-in to decipher functionally grouped gene ontology and pathway annotation networks." Bioinformatics 25(8): 1091-1093.

Blackwood, R. A. and J. D. Ernst (1990). "Characterization of Ca2(+)-dependent phospholipid binding, vesicle aggregation and membrane fusion by annexins." Biochem $\mathrm{J}$ 266(1): 195-200.

Blalock, E. M., H. M. Buechel, J. Popovic, J. W. Geddes and P. W. Landfield (2011). "Microarray analyses of laser-captured hippocampus reveal distinct gray and white matter signatures associated with incipient Alzheimer's disease." $\mathrm{J}$ Chem Neuroanat 42(2): 118-126.

Bliss, T. V. and G. L. Collingridge (1993). "A synaptic model of memory: long-term potentiation in the hippocampus." Nature 361(6407): 31-39.

Bliss, T. V. and T. Lomo (1973). "Long-lasting potentiation of synaptic transmission in the dentate area of the anaesthetized rabbit following stimulation of the perforant path." J Physiol 232(2): 331-356.

Bousiges, O., R. Neidl, M. Majchrzak, M. A. Muller, A. Barbelivien, A. Pereira de Vasconcelos, A. Schneider, J. P. Loeffler, J. C. Cassel and A. L. Boutillier (2013). "Detection of histone acetylation levels in the dorsal hippocampus reveals early tagging on specific residues of $\mathrm{H} 2 \mathrm{~B}$ and $\mathrm{H} 4$ histones in response to learning." PLoS One 8(3): e57816.

Bousiges, O., A. P. Vasconcelos, R. Neidl, B. Cosquer, K. Herbeaux, I. Panteleeva, J. P. Loeffler, J. C. Cassel and A. L. Boutillier (2010). "Spatial memory consolidation is associated with induction of several lysine-acetyltransferase (histone acetyltransferase) expression levels and $\mathrm{H} 2 \mathrm{~B} / \mathrm{H} 4$ acetylation-dependent transcriptional events in the rat hippocampus." Neuropsychopharmacology 35(13): 2521-2537.

Boyko, V., O. Mudrak, M. Svetlova, Y. Negishi, H. Ariga and N. Tomilin (1994). "A major cellular substrate for protein kinases, annexin II, is a DNA-binding protein." FEBS Lett 345(2-3): 139-142.

Braak, H. and E. Braak (1991). "Neuropathological stageing of Alzheimer-related changes." Acta Neuropathol 82(4): 239-259.

Bramblett, G. T., M. Goedert, R. Jakes, S. E. Merrick, J. Q. Trojanowski and V. M. Lee (1993). "Abnormal tau phosphorylation at Ser396 in Alzheimer's disease recapitulates development and contributes to reduced microtubule binding." Neuron 10(6): 1089-1099.

Breen, K. C., M. Bruce and B. H. Anderton (1991). "Beta amyloid precursor protein mediates neuronal cell-cell and cell-surface adhesion." J Neurosci Res 28(1): 90-100. 
Bu, G. (2009). "Apolipoprotein E and its receptors in Alzheimer\&\#39;s disease: pathways, pathogenesis and therapy." Nature Reviews Neuroscience 10: 333.

Calderon-Garciduenas, L., W. Reed, R. R. Maronpot, C. Henriquez-Roldan, R. DelgadoChavez, A. Calderon-Garciduenas, I. Dragustinovis, M. Franco-Lira, M. AragonFlores, A. C. Solt, M. Altenburg, R. Torres-Jardon and J. A. Swenberg (2004). "Brain inflammation and Alzheimer's-like pathology in individuals exposed to severe air pollution." Toxicol Pathol 32(6): 650-658.

Canale, C., S. Seghezza, S. Vilasi, R. Carrotta, D. Bulone, A. Diaspro, P. L. San Biagio and S. Dante (2013). "Different effects of Alzheimer's peptide Abeta(1-40) oligomers and fibrils on supported lipid membranes." Biophys Chem 182: 23-29.

Capone, R., H. Jang, S. A. Kotler, B. L. Kagan, R. Nussinov and R. Lal (2012). "Probing structural features of Alzheimer's amyloid-beta pores in bilayers using site-specific amino acid substitutions." Biochemistry 51(3): 776-785.

Chang, C. H. and D. S. Luse (1997). "The H3/H4 tetramer blocks transcript elongation by RNA polymerase II in vitro." J Biol Chem 272(37): 23427-23434.

Chen, T. and M. Naveh-Benjamin (2012). "Assessing the associative deficit of older adults in long-term and short-term/working memory." Psychol Aging 27(3): 666-682.

Cheung, P., C. D. Allis and P. Sassone-Corsi (2000). "Signaling to chromatin through histone modifications." Cell 103(2): 263-271.

Chow, V. W., M. P. Mattson, P. C. Wong and M. Gleichmann (2010). "An Overview of APP Processing Enzymes and Products." Neuromolecular medicine 12(1): 1-12.

Clavaguera, F., T. Bolmont, R. A. Crowther, D. Abramowski, S. Frank, A. Probst, G. Fraser, A. K. Stalder, M. Beibel, M. Staufenbiel, M. Jucker, M. Goedert and M. Tolnay (2009). "Transmission and spreading of tauopathy in transgenic mouse brain." Nat Cell Biol 11.

Connelly, L., H. Jang, F. T. Arce, R. Capone, S. A. Kotler, S. Ramachandran, B. L. Kagan, R. Nussinov and R. Lal (2012). "Atomic force microscopy and MD simulations reveal pore-like structures of all-D-enantiomer of Alzheimer's beta-amyloid peptide: relevance to the ion channel mechanism of AD pathology." J Phys Chem B 116(5): 1728-1735.

Connelly, L., H. Jang, F. T. Arce, S. Ramachandran, B. L. Kagan, R. Nussinov and R. Lal (2012). "Effects of point substitutions on the structure of toxic Alzheimer's beta-amyloid channels: atomic force microscopy and molecular dynamics simulations." Biochemistry 51(14): 3031-3038.

Cook, C., Y. Carlomagno, T. F. Gendron, J. Dunmore, K. Scheffel, C. Stetler, M. Davis, D. Dickson, M. Jarpe, M. DeTure and L. Petrucelli (2014). "Acetylation of the KXGS motifs in tau is a critical determinant in modulation of tau aggregation and clearance." Hum Mol Genet 23(1): 104-116.

Cook, C., T. F. Gendron, K. Scheffel, Y. Carlomagno, J. Dunmore, M. DeTure and L. Petrucelli (2012). "Loss of HDAC6, a novel CHIP substrate, alleviates abnormal tau accumulation." Hum Mol Genet 21.

Cook, C., T. F. Gendron, K. Scheffel, Y. Carlomagno, J. Dunmore, M. DeTure and L. Petrucelli (2012). "Loss of HDAC6, a novel CHIP substrate, alleviates abnormal tau accumulation." Hum Mol Genet 21(13): 2936-2945. 
Cook, C., J. N. Stankowski, Y. Carlomagno, C. Stetler and L. Petrucelli (2014). "Acetylation: a new key to unlock tau's role in neurodegeneration." Alzheimer's Research \& Therapy 6(3): 29.

Coon, K. D., A. J. Myers, D. W. Craig, J. A. Webster, J. V. Pearson, D. H. Lince, V. L. Zismann, T. G. Beach, D. Leung, L. Bryden, R. F. Halperin, L. Marlowe, M. Kaleem, D. G. Walker, R. Ravid, C. B. Heward, J. Rogers, A. Papassotiropoulos, E. M. Reiman, J. Hardy and D. A. Stephan (2007). "A high-density whole-genome association study reveals that APOE is the major susceptibility gene for sporadic late-onset Alzheimer's disease." J Clin Psychiatry 68(4): 613-618.

Copenhaver, P. F. and J. M. Ramaker (2016). "Neuronal migration during development and the amyloid precursor protein." Curr Opin Insect Sci 18: 1-10.

Cosgrove, M. S., J. D. Boeke and C. Wolberger (2004). "Regulated nucleosome mobility and the histone code." Nat Struct Mol Biol 11(11): 1037-1043.

Cowan, N. (2008). Chapter 20 What are the differences between long-term, short-term, and working memory? Essence of Memory: 323-338.

Cripps, D., S. N. Thomas, Y. Jeng, F. Yang, P. Davies and A. J. Yang (2006). "Alzheimer disease-specific conformation of hyperphosphorylated paired helical filament-Tau is polyubiquitinated through Lys-48, Lys-11, and Lys-6 ubiquitin conjugation." $\underline{\mathrm{J} \text { Biol }}$ Chem 281.

Cui, Z., C. R. Gerfen and W. S. Young, 3rd (2013). "Hypothalamic and other connections with dorsal CA2 area of the mouse hippocampus." J Comp Neurol 521(8): 1844-1866.

da Motta, L. L., I. Ledaki, K. Purshouse, S. Haider, M. A. De Bastiani, D. Baban, M. Morotti, G. Steers, S. Wigfield, E. Bridges, J. L. Li, S. Knapp, D. Ebner, F. Klamt, A. L. Harris and A. McIntyre (2017). "The BET inhibitor JQ1 selectively impairs tumour response to hypoxia and downregulates $\mathrm{CA} 9$ and angiogenesis in triple negative breast cancer." Oncogene 36(1): 122-132.

Dale, N. and E. R. Kandel (1993). "L-glutamate may be the fast excitatory transmitter of Aplysia sensory neurons." Proc Natl Acad Sci U S A 90(15): 7163-7167.

Das, C. and J. K. Tyler (2013). "Histone exchange and histone modifications during transcription and aging()." Biochimica et biophysica acta 1819(0): 332-342.

Das, S., P. Shetty, M. Valapala, S. Dasgupta, Z. Gryczynski and J. K. Vishwanatha (2010). "Signal transducer and activator of transcription 6 (STAT6) is a novel interactor of annexin A2 in prostate cancer cells." Biochemistry 49(10): 2216-2226.

Dash, P. K., B. Hochner and E. R. Kandel (1990). "Injection of the cAMP-responsive element into the nucleus of Aplysia sensory neurons blocks long-term facilitation." Nature 345(6277): 718-721.

Dauth, S., R. F. Sirbulescu, S. Jordans, M. Rehders, L. Avena, J. Oswald, A. Lerchl, P. Saftig and K. Brix (2011). "Cathepsin K deficiency in mice induces structural and metabolic changes in the central nervous system that are associated with learning and memory deficits." BMC Neurosci 12: 74.

Davie, J. R. and L. C. Murphy (1990). "Level of ubiquitinated histone H2B in chromatin is coupled to ongoing transcription." Biochemistry 29(20): 4752-4757.

de Calignon, A., M. Polydoro, M. Suarez-Calvet, C. William, D. H. Adamowicz, K. J. Kopeikina, R. Pitstick, N. Sahara, K. H. Ashe, G. A. Carlson, T. L. Spires-Jones and B. T. 
Hyman (2012). "Propagation of tau pathology in a model of early Alzheimer's disease." Neuron 73(4): 685-697.

de Ruijter, A. J., A. H. van Gennip, H. N. Caron, S. Kemp and A. B. van Kuilenburg (2003). "Histone deacetylases (HDACs): characterization of the classical HDAC family." Biochem J 370(Pt 3): 737-749.

Deeg, A. A., A. M. Reiner, F. Schmidt, F. Schueder, S. Ryazanov, V. C. Ruf, K. Giller, S. Becker, A. Leonov, C. Griesinger, A. Giese and W. Zinth (2015). "Anle138b and related compounds are aggregation specific fluorescence markers and reveal high affinity binding to alpha-synuclein aggregates." Biochim Biophys Acta 1850(9): 1884-1890.

Deora, A. B., G. Kreitzer, A. T. Jacovina and K. A. Hajjar (2004). "An annexin 2 phosphorylation switch mediates p11-dependent translocation of annexin 2 to the cell surface." $\underline{\mathrm{J}}$ Biol Chem 279(42): 43411-43418.

Derbyshire, Z. E., U. M. Halfter, R. L. Heimark, T. H. Sy and R. R. Vaillancourt (2005). "Angiotensin II stimulated transcription of cyclooxygenase II is regulated by a novel kinase cascade involving Pyk2, MEKK4 and annexin II." Mol Cell Biochem 271(1-2): 77-90.

Dey, A., F. Chitsaz, A. Abbasi, T. Misteli and K. Ozato (2003). "The double bromodomain protein Brd4 binds to acetylated chromatin during interphase and mitosis." Proc Natl Acad Sci U S A 100(15): 8758-8763.

Dhalluin, C., J. E. Carlson, L. Zeng, C. He, A. K. Aggarwal and M. M. Zhou (1999). "Structure and ligand of a histone acetyltransferase bromodomain." Nature 399(6735): 491-496.

Dickson, D. W. (1997). "Neuropathological diagnosis of Alzheimer's disease: a perspective from longitudinal clinicopathological studies." Neurobiol Aging 18(4 Suppl): S21-26.

Dickson, D. W., N. Kouri, M. E. Murray and K. A. Josephs (2011). "Neuropathology of frontotemporal lobar degeneration-tau (FTLD-tau)." J Mol Neurosci 45(3): 384-389.

Ding, H., P. J. Dolan and G. V. Johnson (2008). "Histone deacetylase 6 interacts with the microtubule-associated protein tau." J Neurochem 106(5): 2119-2130.

Ding, H., P. J. Dolan and G. V. W. Johnson (2008). "Histone deacetylase 6 interacts with the microtubule-associated protein tau." Journal of neurochemistry 106(5): 2119-2130.

Dityatev, A. and M. Schachner (2003). "Extracellular matrix molecules and synaptic plasticity." Nature Reviews Neuroscience 4: 456.

Dobin, A., C. A. Davis, F. Schlesinger, J. Drenkow, C. Zaleski, S. Jha, P. Batut, M. Chaisson and T. R. Gingeras (2013). "STAR: ultrafast universal RNA-seq aligner." Bioinformatics 29(1): 15-21.

Doig, A. J. and P. Derreumaux (2015). "Inhibition of protein aggregation and amyloid formation by small molecules." Curr Opin Struct Biol 30: 50-56.

Dompierre, J. P., J. D. Godin, B. C. Charrin, F. P. Cordelieres, S. J. King, S. Humbert and F. Saudou (2007). "Histone deacetylase 6 inhibition compensates for the transport

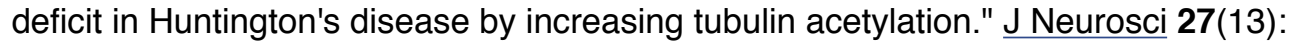
3571-3583.

Dorval, V. and P. E. Fraser (2006). "Small ubiquitin-like modifier (SUMO) modification of natively unfolded proteins tau and alpha-synuclein." J Biol Chem 281(15): 9919-9924. 
Drechsel, D. N., A. A. Hyman, M. H. Cobb and M. W. Kirschner (1992). "Modulation of the dynamic instability of tubulin assembly by the microtubule-associated protein tau." Mol Biol Cell 3(10): 1141-1154.

Drubin, D. G. and M. W. Kirschner (1986). "Tau protein function in living cells." J Cell Biol 103(6 Pt 2): 2739-2746.

Drucker, P., M. Pejic, H. J. Galla and V. Gerke (2013). "Lipid segregation and membrane budding induced by the peripheral membrane binding protein annexin A2." $\underline{\mathrm{J} \text { Biol }}$ Chem 288(34): 24764-24776.

Dudai, Y. (1996). "Consolidation: fragility on the road to the engram." Neuron 17(3): 367-370.

Dudai, Y. (2002). "Memory from A to Z keywords, concepts, and beyon."

Eberhard, D. A., L. R. Karns, S. R. VandenBerg and C. E. Creutz (2001). "Control of the nuclear-cytoplasmic partitioning of annexin II by a nuclear export signal and by $\mathrm{p} 11$ binding." J Cell Sci 114(Pt 17): 3155-3166.

Ennaceur, A. and J. Delacour (1988). "A new one-trial test for neurobiological studies of memory in rats. 1: Behavioral data." Behavioural Brain Research 31(1): 47-59.

Ezkurdia, I., D. Juan, J. M. Rodriguez, A. Frankish, M. Diekhans, J. Harrow, J. Vazquez, A. Valencia and M. L. Tress (2014). "Multiple evidence strands suggest that there may be as few as 19000 human protein-coding genes." Human Molecular Genetics 23(22): 5866-5878.

Farr, S. A., K. Uezu, J. F. Flood and J. E. Morley (1999). "Septo-hippocampal drug interactions in post-trial memory processing." Brain Res 847(2): 221-230.

Fellner, L., D. Kuzdas-Wood, J. Levin, S. Ryazanov, A. Leonov, C. Griesinger, A. Giese, G. K. Wenning and N. Stefanova (2016). "Anle138b Partly Ameliorates Motor Deficits Despite Failure of Neuroprotection in a Model of Advanced Multiple System Atrophy." Front Neurosci 10: 99.

Filbin, M. E. and J. S. Kieft (2009). "Toward a structural understanding of IRES RNA function." Curr Opin Struct Biol 19(3): 267-276.

Filipenko, N. R., T. J. MacLeod, C. S. Yoon and D. M. Waisman (2004). "Annexin A2 is a novel RNA-binding protein." J Biol Chem 279(10): 8723-8731.

Filippakopoulos, P. and S. Knapp (2012). "The bromodomain interaction module." FEBS Lett 586(17): 2692-2704.

Fischer, A. (2014). "Epigenetic memory: the Lamarckian brain." EMBO J 33(9): 945-967.

Fischer, A. (2014). "Targeting histone-modifications in Alzheimer's disease. What is the evidence that this is a promising therapeutic avenue?" Neuropharmacology 80: 95-102.

Fischer, A., F. Sananbenesi, A. Mungenast and L. H. Tsai (2010). "Targeting the correct HDAC(s) to treat cognitive disorders." Trends Pharmacol Sci 31(12): 605-617.

Fischer, A., F. Sananbenesi, X. Wang, M. Dobbin and L. H. Tsai (2007). "Recovery of learning and memory is associated with chromatin remodelling." Nature 447(7141): 178-182.

Flexner, J. B., L. B. Flexner, E. Stellar, G. De La Haba and R. B. Roberts (1962). "Inhibition of protein synthesis in brain and learning and memory following puromycin." $\underline{\mathrm{J}}$ Neurochem 9: 595-605. 
Flood, J. F., M. R. Rosenzweig, E. L. Bennett and A. E. Orme (1973). "The influence of duration of protein synthesis inhibition on memory." Physiol Behav 10(3): 555-562.

Fontan-Lozano, A., R. Romero-Granados, J. Troncoso, A. Munera, J. M. Delgado-Garcia and A. M. Carrion (2008). "Histone deacetylase inhibitors improve learning consolidation in young and in KA-induced-neurodegeneration and SAMP-8-mutant mice." Mol Cell Neurosci 39(2): 193-201.

Forstl, H. and A. Kurz (1999). "Clinical features of Alzheimer's disease." Eur Arch Psychiatry Clin Neurosci 249(6): 288-290.

Frey, U. and R. G. Morris (1998). "Synaptic tagging: implications for late maintenance of hippocampal long-term potentiation." Trends Neurosci 21(5): 181-188.

Friedhoff, P., M. von Bergen, E. M. Mandelkow, P. Davies and E. Mandelkow (1998). "A nucleated assembly mechanism of Alzheimer paired helical filaments." Proceedings of the National Academy of Sciences of the United States of America 95(26): 15712-15717.

Gabel, M., F. Delavoie, V. Demais, C. Royer, Y. Bailly, N. Vitale, M. F. Bader and S. ChasserotGolaz (2015). "Annexin A2-dependent actin bundling promotes secretory granule docking to the plasma membrane and exocytosis." J Cell Biol 210(5): 785-800.

Gauthier-Kemper, A., C. Weissmann, N. Golovyashkina, Z. Sebo-Lemke, G. Drewes, V. Gerke, J. J. Heinisch and R. Brandt (2011). "The frontotemporal dementia mutation R406W blocks tau's interaction with the membrane in an annexin A2-dependent manner." J Cell Biol 192(4): 647-661.

Gerhardt, K. P., E. J. Olson, S. M. Castillo-Hair, L. A. Hartsough, B. P. Landry, F. Ekness, R. Yokoo, E. J. Gomez, P. Ramakrishnan, J. Suh, D. F. Savage and J. J. Tabor (2016). "An open-hardware platform for optogenetics and photobiology." Sci Rep 6: 35363.

Gerke, V., C. E. Creutz and S. E. Moss (2005). "Annexins: linking Ca2+ signalling to membrane dynamics." Nat Rev Mol Cell Biol 6(6): 449-461.

Gerke, V. and S. E. Moss (2002). "Annexins: from structure to function." Physiol Rev 82(2): 331-371.

Gibbs, M. E. and K. T. Ng (1977). "Counteractive effects of norepinephrine and amphetamine on quabain-induced amnesia." Pharmacol Biochem Behav 6(5): 533-537.

Goate, A. and J. Hardy (2012). "Twenty years of Alzheimer's disease-causing mutations." $\underline{J}$ Neurochem 120 Suppl 1: 3-8.

Goedert, M. and R. Jakes (1990). "Expression of separate isoforms of human tau protein: correlation with the tau pattern in brain and effects on tubulin polymerization." EMBO J 9(13): 4225-4230.

Goedert, M., M. Masuda-Suzukake and B. Falcon (2017). "Like prions: the propagation of aggregated tau and alpha-synuclein in neurodegeneration." Brain 140(2): 266-278.

Goedert, M., M. G. Spillantini, M. C. Potier, J. Ulrich and R. A. Crowther (1989). "Cloning and sequencing of the cDNA encoding an isoform of microtubule-associated protein tau containing four tandem repeats: differential expression of tau protein mRNAs in human brain." EMBO J 8(2): 393-399.

Goelet, P., V. F. Castellucci, S. Schacher and E. R. Kandel (1986). "The long and the short of long-term memory--a molecular framework." Nature 322(6078): 419-422. 
Gold, M. (2017). "Phase II clinical trials of anti-amyloid beta antibodies: When is enough, enough?" Alzheimers Dement (N Y) 3(3): 402-409.

Gold, P. E. (2008). "Protein synthesis and memory." Neurobiology of learning and memory 89(3): 199-200.

Goldgaber, D., M. I. Lerman, O. W. McBride, U. Saffiotti and D. C. Gajdusek (1987). "Characterization and chromosomal localization of a cDNA encoding brain amyloid of Alzheimer's disease." Science 235(4791): 877-880.

Gomez-Ramos, P. and M. Asuncion Moran (2007). "Ultrastructural localization of intraneuronal Abeta-peptide in Alzheimer disease brains." J Alzheimers Dis 11(1): 53-59.

Gong, C. X., I. Grundke-lqbal and K. Iqbal (1994). "Dephosphorylation of Alzheimer's disease abnormally phosphorylated tau by protein phosphatase-2A." Neuroscience 61(4): 765-772.

Govindarajan, N., P. Rao, S. Burkhardt, F. Sananbenesi, O. M. Schluter, F. Bradke, J. Lu and A. Fischer (2013). "Reducing HDAC6 ameliorates cognitive deficits in a mouse model for Alzheimer's disease." EMBO Mol Med 5(1): 52-63.

Grindheim, A. K., H. Hollas, A. M. Raddum, J. Saraste and A. Vedeler (2016). "Reactive oxygen species exert opposite effects on Tyr23 phosphorylation of the nuclear and cortical pools of annexin A2." J Cell Sci 129(2): 314-328.

Grindheim, A. K., H. Hollas, J. Ramirez, J. Saraste, G. Trave and A. Vedeler (2014). "Effect of serine phosphorylation and Ser25 phospho-mimicking mutations on nuclear localisation and ligand interactions of annexin A2." J Mol Biol 426(13): 2486-2499.

Grindheim, A. K., J. Saraste and A. Vedeler (2017). "Protein phosphorylation and its role in the regulation of Annexin A2 function." Biochim Biophys Acta 1861(11 Pt A): 2515-2529.

Grossman, N., V. Poher, M. S. Grubb, G. T. Kennedy, K. Nikolic, B. McGovern, R. Berlinguer Palmini, Z. Gong, E. M. Drakakis, M. A. Neil, M. D. Dawson, J. Burrone and P. Degenaar (2010). "Multi-site optical excitation using ChR2 and micro-LED array." $\underline{\mathrm{J}}$ Neural Eng 7(1): 16004.

Grundke-lqbal, I., K. Iqbal, Y. C. Tung, M. Quinlan, H. M. Wisniewski and L. I. Binder (1986). "Abnormal phosphorylation of the microtubule-associated protein tau (tau) in Alzheimer cytoskeletal pathology." Proc Natl Acad Sci USA 83.

Guan, J. S., S. J. Haggarty, E. Giacometti, J. H. Dannenberg, N. Joseph, J. Gao, T. J. Nieland, Y. Zhou, X. Wang, R. Mazitschek, J. E. Bradner, R. A. DePinho, R. Jaenisch and L. $\mathrm{H}$. Tsai (2009). "HDAC2 negatively regulates memory formation and synaptic plasticity." Nature 459(7243): 55-60.

Guan, Z., M. Giustetto, S. Lomvardas, J. H. Kim, M. C. Miniaci, J. H. Schwartz, D. Thanos and E. R. Kandel (2002). "Integration of long-term-memory-related synaptic plasticity involves bidirectional regulation of gene expression and chromatin structure." Cell 111(4): 483-493.

Guo, Z., L. A. Cupples, A. Kurz, S. H. Auerbach, L. Volicer, H. Chui, R. C. Green, A. D. Sadovnick, R. Duara, C. DeCarli, K. Johnson, R. C. Go, J. H. Growdon, J. L. Haines, W. A. Kukull and L. A. Farrer (2000). "Head injury and the risk of AD in the MIRAGE study." Neurology 54(6): 1316-1323.

Gustafson, D., E. Rothenberg, K. Blennow, B. Steen and I. Skoog (2003). "An 18-year follow-up of overweight and risk of Alzheimer disease." Arch Intern Med 163(13): 1524-1528. 
Haass, C. and D. J. Selkoe (2007). "Soluble protein oligomers in neurodegeneration: lessons from the Alzheimer's amyloid beta-peptide." Nat Rev Mol Cell Biol 8(2): 101-112.

Hall, C. S. (1934). "Emotional behavior in the rat. I. Defecation and urination as measures of individual differences in emotionality." Journal of Comparative Psychology 18(3): 385-403.

Hallmann, A. L., M. J. Arauzo-Bravo, L. Mavrommatis, M. Ehrlich, A. Ropke, J. Brockhaus, M. Missler, J. Sterneckert, H. R. Scholer, T. Kuhlmann, H. Zaehres and G. Hargus (2017). "Astrocyte pathology in a human neural stem cell model of frontotemporal dementia caused by mutant TAU protein." Sci Rep 7: 42991.

Handa, A., S.-i. Muramatsu, J. Qiu, H. Mizukami and K. E. Brown (2000). "Adeno-associated virus (AAV)-3-based vectors transduce haematopoietic cells not susceptible to transduction with AAV-2-based vectors." Journal of General Virology 81(8): 2077-2084.

Harada, A., K. Oguchi, S. Okabe, J. Kuno, S. Terada, T. Ohshima, R. Sato-Yoshitake, Y. Takei, T. Noda and N. Hirokawa (1994). "Altered microtubule organization in small-calibre axons of mice lacking tau protein." Nature 369(6480): 488-491.

Hardy, J. and D. J. Selkoe (2002). "The amyloid hypothesis of Alzheimer's disease: progress and problems on the road to therapeutics." Science 297(5580): 353-356.

Harrison, F. E., A. H. Hosseini and M. P. McDonald (2009). "Endogenous anxiety and stress responses in water maze and Barnes maze spatial memory tasks." Behav Brain Res 198(1): 247-251.

Hasegawa, M., M. Morishima-Kawashima, K. Takio, M. Suzuki, K. Titani and Y. Ihara (1992). "Protein sequence and mass spectrometric analyses of tau in the Alzheimer's disease brain." J Biol Chem 267.

Hebbes, T. R., A. L. Clayton, A. W. Thorne and C. Crane-Robinson (1994). "Core histone hyperacetylation co-maps with generalized DNase I sensitivity in the chicken betaglobin chromosomal domain." EMBO J 13(8): 1823-1830.

Hebert, L. E., J. Weuve, P. A. Scherr and D. A. Evans (2013). "Alzheimer disease in the United States (2010-2050) estimated using the 2010 census." Neurology 80(19): 1778-1783.

Hempen, B. and J. P. Brion (1996). "Reduction of acetylated alpha-tubulin immunoreactivity in neurofibrillary tangle-bearing neurons in Alzheimer's disease." J Neuropathol Exp Neurol 55(9): 964-972.

Hendrickx, A., N. Pierrot, B. Tasiaux, O. Schakman, P. Kienlen-Campard, C. De Smet and J. N. Octave (2014). "Epigenetic regulations of immediate early genes expression involved in memory formation by the amyloid precursor protein of Alzheimer disease." PLoS One 9(6): e99467.

Hitchcock, J. K., A. A. Katz and G. Schafer (2014). "Dynamic reciprocity: the role of annexin A2 in tissue integrity." J Cell Commun Signal 8(2): 125-133.

Hnilicova, J., S. Hozeifi, E. Duskova, J. Icha, T. Tomankova and D. Stanek (2011). "Histone deacetylase activity modulates alternative splicing." PLoS One 6(2): e16727.

Hollas, H., I. Aukrust, S. Grimmer, E. Strand, T. Flatmark and A. Vedeler (2006). "Annexin A2 recognises a specific region in the 3'-UTR of its cognate messenger RNA." Biochim Biophys Acta 1763(11): 1325-1334. 
Hou, Y., L. Yang, M. Mou, Y. Hou, A. Zhang, N. Pan, R. Qiang, L. Wei and N. Zhang (2008).

"Annexin A2 regulates the levels of plasmin, S100A10 and Fascin in L5178Y cells." Cancer Invest 26(8): 809-815.

Hubbert, C., A. Guardiola, R. Shao, Y. Kawaguchi, A. Ito, A. Nixon, M. Yoshida, X. F. Wang and T. P. Yao (2002). "HDAC6 is a microtubule-associated deacetylase." Nature 417(6887): 455-458.

Hussong, M., C. Kaehler, M. Kerick, C. Grimm, A. Franz, B. Timmermann, F. Welzel, J. Isensee, T. Hucho, S. Krobitsch and M. R. Schweiger (2017). "The bromodomain protein BRD4 regulates splicing during heat shock." Nucleic Acids Res 45(1): 382-394.

Im, H. I. and P. J. Kenny (2012). "MicroRNAs in neuronal function and dysfunction." Trends Neurosci 35(5): 325-334.

international, A. s. D. (2016). "World Alzheimer Report 2016." World Alzheimer Report.

International Human Genome Sequencing, C. (2004). "Finishing the euchromatic sequence of the human genome." Nature 431: 931.

Iqbal, K., H. M. Wiśniewski, M. L. Shelanski, S. Brostoff, B. H. Liwnicz and R. D. Terry (1974). "Protein changes in senile dementia." Brain Research 77(2): 337-343.

Iwatsubo, T., A. Odaka, N. Suzuki, H. Mizusawa, N. Nukina and Y. Ihara (1994). "Visualization of $A \beta 42(43)$ and $A \beta 40$ in senile plaques with end-specific $A \beta$ monoclonals: evidence that an initially deposited species is $A \beta 42(43) . "$ Neuron 13(1): 45-53.

Izquierdo, J. M. (2008). "Hu antigen R (HuR) functions as an alternative pre-mRNA splicing regulator of Fas apoptosis-promoting receptor on exon definition." J Biol Chem 283(27): 19077-19084.

Jaitin, D. A., E. Kenigsberg, H. Keren-Shaul, N. Elefant, F. Paul, I. Zaretsky, A. Mildner, N. Cohen, S. Jung, A. Tanay and I. Amit (2014). "Massively parallel single-cell RNAseq for marker-free decomposition of tissues into cell types." Science 343(6172): 776-779.

Jenuwein, T. and C. D. Allis (2001). "Translating the histone code." Science 293(5532): 1074-1080.

Jeon, B. N., W. I. Choi, M. Y. Yu, A. R. Yoon, M. H. Kim, C. O. Yun and M. W. Hur (2009). "ZBTB2, a novel master regulator of the p53 pathway." J Biol Chem 284(27): 17935-17946.

Jindal, H. K., W. G. Chaney, C. W. Anderson, R. G. Davis and J. K. Vishwanatha (1991). "The protein-tyrosine kinase substrate, calpactin I heavy chain (p36), is part of the primer recognition protein complex that interacts with DNA polymerase alpha." $\underline{\mathrm{J}}$ Biol Chem 266(8): 5169-5176.

Johnson, C. A. and B. M. Turner (1999). "Histone deacetylases: complex transducers of nuclear signals." Semin Cell Dev Biol 10(2): 179-188.

Jonsson, T., J. K. Atwal, S. Steinberg, J. Snaedal, P. V. Jonsson, S. Bjornsson, H. Stefansson, P. Sulem, D. Gudbjartsson, J. Maloney, K. Hoyte, A. Gustafson, Y. Liu, Y. Lu, T. Bhangale, R. R. Graham, J. Huttenlocher, G. Bjornsdottir, O. A. Andreassen, E. G. Jonsson, A. Palotie, T. W. Behrens, O. T. Magnusson, A. Kong, U. Thorsteinsdottir, R. J. Watts and K. Stefansson (2012). "A mutation in APP protects against Alzheimer's disease and age-related cognitive decline." Nature 488(7409): 96-99.

Jung, M., M. Philpott, S. Müller, J. Schulze, V. Badock, U. Eberspächer, D. Moosmayer, B. Bader, N. Schmees, A. Fernández-Montalván and B. Haendler (2014). "Affinity 
Map of Bromodomain Protein 4 (BRD4) Interactions with the Histone H4 Tail and the Small Molecule Inhibitor JQ1." The Journal of Biological Chemistry 289(13): 9304-9319.

Kadavath, H., R. V. Hofele, J. Biernat, S. Kumar, K. Tepper, H. Urlaub, E. Mandelkow and M. Zweckstetter (2015). "Tau stabilizes microtubules by binding at the interface between tubulin heterodimers." Proc Natl Acad Sci U S A 112(24): 7501-7506.

Kahn, I. and D. Shohamy (2013). "Intrinsic connectivity between the hippocampus, nucleus accumbens, and ventral tegmental area in humans." Hippocampus 23(3): 187-192.

Kaludov, N., K. E. Brown, R. W. Walters, J. Zabner and J. A. Chiorini (2001). "Adeno-associated virus serotype 4 (AAV4) and AAV5 both require sialic acid binding for hemagglutination and efficient transduction but differ in sialic acid linkage specificity." J Virol 75(15): 6884-6893.

Kamakaka, R. T. and S. Biggins (2005). "Histone variants: deviants?" Genes Dev 19(3): 295-310.

Kandel, E. R. (2001). "The molecular biology of memory storage: a dialogue between genes and synapses." Science 294(5544): 1030-1038.

Kandel, E. R., Y. Dudai and M. R. Mayford (2014). "The molecular and systems biology of memory." Cell 157(1): 163-186.

Kang, J., H. G. Lemaire, A. Unterbeck, J. M. Salbaum, C. L. Masters, K. H. Grzeschik, G. Multhaup, K. Beyreuther and B. Muller-Hill (1987). "The precursor of Alzheimer's disease amyloid A4 protein resembles a cell-surface receptor." Nature 325(6106): 733-736.

Kanno, T., Y. Kanno, R. M. Siegel, M. K. Jang, M. J. Lenardo and K. Ozato (2004). "Selective recognition of acetylated histones by bromodomain proteins visualized in living cells." Mol Cell 13(1): 33-43.

Kern, A., K. Schmidt, C. Leder, O. J. Müller, C. E. Wobus, K. Bettinger, C. W. Von der Lieth, J. A. King and J. A. Kleinschmidt (2003). "Identification of a Heparin-Binding Motif on Adeno-Associated Virus Type 2 Capsids." Journal of Virology 77(20): 11072-11081.

Khatoon, S., I. Grundke-Iqbal and K. Iqbal (1992). "Brain levels of microtubule-associated protein tau are elevated in Alzheimer's disease: a radioimmuno-slot-blot assay for nanograms of the protein." J Neurochem 59(2): 750-753.

Kim, C., H. Choi, E. S. Jung, W. Lee, S. Oh, N. L. Jeon and I. Mook-Jung (2012). "HDAC6 inhibitor blocks amyloid beta-induced impairment of mitochondrial transport in hippocampal neurons." PLoS One 7(8): e42983.

Kim, K. H., M. Moon, S. B. Yu, I. Mook-Jung and J. I. Kim (2012). "RNA-Seq analysis of frontal cortex and cerebellum from 5XFAD mice at early stage of disease pathology." $\underline{J}$ Alzheimers Dis 29(4): 793-808.

Kim, M. Y., D. I. Koh, W. I. Choi, B. N. Jeon, D. Y. Jeong, K. S. Kim, K. Kim, S. H. Kim and M. W. Hur (2015). "ZBTB2 increases PDK4 expression by transcriptional repression of RelA/p65." Nucleic Acids Res 43(3): 1609-1625.

Kitamura, T., S. K. Ogawa, D. S. Roy, T. Okuyama, M. D. Morrissey, L. M. Smith, R. L. Redondo and S. Tonegawa (2017). "Engrams and Circuits Crucial for Systems Consolidation of a Memory." Science (New York, N.Y.) 356(6333): 73-78.

Klevanski, M., U. Herrmann, S. W. Weyer, R. Fol, N. Cartier, D. P. Wolfer, J. H. Caldwell, M. Korte and U. C. Muller (2015). "The APP Intracellular Domain Is Required for 
Normal Synaptic Morphology, Synaptic Plasticity, and Hippocampus-Dependent Behavior." J Neurosci 35(49): 16018-16033.

Kokawa, A., S. Ishihara, H. Fujiwara, M. Nobuhara, M. Iwata, Y. Ihara and S. Funamoto (2015). "The A673T mutation in the amyloid precursor protein reduces the production of beta-amyloid protein from its beta-carboxyl terminal fragment in cells." Acta Neuropathol Commun 3: 66.

Kopke, E., Y. C. Tung, S. Shaikh, A. C. Alonso, K. Iqbal and I. Grundke-lqbal (1993). "Microtubule-associated protein tau. Abnormal phosphorylation of a non-paired helical filament pool in Alzheimer disease." J Biol Chem 268(32): 24374-24384.

Korb, E., M. Herre, I. Zucker-Scharff, R. B. Darnell and C. D. Allis (2015). "BET protein Brd4 activates transcription in neurons and BET inhibitor Jq1 blocks memory in mice." Nat Neurosci 18(10): 1464-1473.

Kornberg, R. D. and Y. Lorch (1999). "Twenty-five years of the nucleosome, fundamental particle of the eukaryote chromosome." Cell 98(3): 285-294.

Korzus, E., M. G. Rosenfeld and M. Mayford (2004). "CBP histone acetyltransferase activity is a critical component of memory consolidation." Neuron 42(6): 961-972.

Kosarussavadi, S., Z. T. Pennington, J. Covell, A. P. Blaisdell and B. A. Schlinger (2017). "Across sex and age: Learning and memory and patterns of avian hippocampal gene expression." Behav Neurosci 131(6): 483-491.

Koshibu, K., J. Graff, M. Beullens, F. D. Heitz, D. Berchtold, H. Russig, M. Farinelli, M. Bollen and I. M. Mansuy (2009). "Protein phosphatase 1 regulates the histone code for long-term memory." J Neurosci 29(41): 13079-13089.

Krajewski, W. A. and P. B. Becker (1998). "Reconstitution of hyperacetylated, DNase I-sensitive chromatin characterized by high conformational flexibility of nucleosomal DNA." Proc Natl Acad Sci U S A 95(4): 1540-1545.

Kumble, K. D., P. L. Iversen and J. K. Vishwanatha (1992). "The role of primer recognition proteins in DNA replication: inhibition of cellular proliferation by antisense oligodeoxyribonucleotides." J Cell Sci 101 ( Pt 1): 35-41.

Kummer, M. P., T. Hammerschmidt, A. Martinez, D. Terwel, G. Eichele, A. Witten, S. Figura, M. Stoll, S. Schwartz, H. C. Pape, J. L. Schultze, D. Weinshenker, M. T. Heneka and I. Urban (2014). "Ear2 deletion causes early memory and learning deficits in APP/ PS1 mice." J Neurosci 34(26): 8845-8854.

Kwak, H., N. J. Fuda, L. J. Core and J. T. Lis (2013). "Precise Maps of RNA Polymerase Reveal How Promoters Direct Initiation and Pausing." Science (New York, N.Y.) 339(6122): 950-953.

Kwak, H., M. W. Park and S. Jeong (2011). "Annexin A2 binds RNA and reduces the frameshifting efficiency of infectious bronchitis virus." PLoS One 6(8): e24067.

Lacor, P. N., M. C. Buniel, P. W. Furlow, A. S. Clemente, P. T. Velasco, M. Wood, K. L. Viola and W. L. Klein (2007). "Abeta oligomer-induced aberrations in synapse composition, shape, and density provide a molecular basis for loss of connectivity in Alzheimer's disease." J Neurosci 27(4): 796-807.

Lander, E. S., L. M. Linton, B. Birren, C. Nusbaum, M. C. Zody, J. Baldwin, K. Devon, K. Dewar, M. Doyle, W. FitzHugh, R. Funke, D. Gage, K. Harris, A. Heaford, J. Howland, L. Kann, J. Lehoczky, R. LeVine, P. McEwan, K. McKernan, J. Meldrim, J. P. Mesirov, C. Miranda, W. Morris, J. Naylor, C. Raymond, M. Rosetti, R. Santos, A. Sheridan, 
C. Sougnez, Y. Stange-Thomann, N. Stojanovic, A. Subramanian, D. Wyman, J. Rogers, J. Sulston, R. Ainscough, S. Beck, D. Bentley, J. Burton, C. Clee, N. Carter, A. Coulson, R. Deadman, P. Deloukas, A. Dunham, I. Dunham, R. Durbin, L. French, D. Grafham, S. Gregory, T. Hubbard, S. Humphray, A. Hunt, M. Jones, C. Lloyd, A. McMurray, L. Matthews, S. Mercer, S. Milne, J. C. Mullikin, A. Mungall, R. Plumb, M. Ross, R. Shownkeen, S. Sims, R. H. Waterston, R. K. Wilson, L. W. Hillier, J. D. McPherson, M. A. Marra, E. R. Mardis, L. A. Fulton, A. T. Chinwalla, K. H. Pepin, W. R. Gish, S. L. Chissoe, M. C. Wendl, K. D. Delehaunty, T. L. Miner, A. Delehaunty, J. B. Kramer, L. L. Cook, R. S. Fulton, D. L. Johnson, P. J. Minx, S. W. Clifton, T. Hawkins, E. Branscomb, P. Predki, P. Richardson, S. Wenning, T. Slezak, N. Doggett, J. F. Cheng, A. Olsen, S. Lucas, C. Elkin, E. Uberbacher, M. Frazier, R. A. Gibbs, D. M. Muzny, S. E. Scherer, J. B. Bouck, E. J. Sodergren, K. C. Worley, C. M. Rives, J. H. Gorrell, M. L. Metzker, S. L. Naylor, R. S. Kucherlapati, D. L. Nelson, G. M. Weinstock, Y. Sakaki, A. Fujiyama, M. Hattori, T. Yada, A. Toyoda, T. Itoh, C. Kawagoe, H. Watanabe, Y. Totoki, T. Taylor, J. Weissenbach, R. Heilig, W. Saurin, F. Artiguenave, P. Brottier, T. Bruls, E. Pelletier, C. Robert, P. Wincker, D. R. Smith, L. Doucette-Stamm, M. Rubenfield, K. Weinstock, H. M. Lee, J. Dubois, A. Rosenthal, M. Platzer, G. Nyakatura, S. Taudien, A. Rump, H. Yang, J. Yu, J. Wang, G. Huang, J. Gu, L. Hood, L. Rowen, A. Madan, S. Qin, R. W. Davis, N. A. Federspiel, A. P. Abola, M. J. Proctor, R. M. Myers, J. Schmutz, M. Dickson, J. Grimwood, D. R. Cox, M. V. Olson, R. Kaul, C. Raymond, N. Shimizu, K. Kawasaki, S. Minoshima, G. A. Evans, M. Athanasiou, R. Schultz, B. A. Roe, F. Chen, H. Pan, J. Ramser, H. Lehrach, R. Reinhardt, W. R. McCombie, M. de la Bastide, N. Dedhia, H. Blocker, K. Hornischer, G. Nordsiek, R. Agarwala, L. Aravind, J. A. Bailey, A. Bateman, S. Batzoglou, E. Birney, P. Bork, D. G. Brown, C. B. Burge, L. Cerutti, H. C. Chen, D. Church, M. Clamp, R. R. Copley, T. Doerks, S. R. Eddy, E. E. Eichler, T. S. Furey, J. Galagan, J. G. Gilbert, C. Harmon, Y. Hayashizaki, D. Haussler, H. Hermjakob, K. Hokamp, W. Jang, L. S. Johnson, T. A. Jones, S. Kasif, A. Kaspryzk, S. Kennedy, W. J. Kent, P. Kitts, E. V. Koonin, I. Korf, D. Kulp, D. Lancet, T. M. Lowe, A. McLysaght, T. Mikkelsen, J. V. Moran, N. Mulder, V. J. Pollara, C. P. Ponting, G. Schuler, J. Schultz, G. Slater, A. F. Smit, E. Stupka, J. Szustakowki, D. Thierry-Mieg, J. Thierry-Mieg, L. Wagner, J. Wallis, R. Wheeler, A. Williams, Y. I. Wolf, K. H. Wolfe, S. P. Yang, R. F. Yeh, F. Collins, M. S. Guyer, J. Peterson, A. Felsenfeld, K. A. Wetterstrand, A. Patrinos, M. J. Morgan, P. de Jong, J. J. Catanese, K. Osoegawa, H. Shizuya, S. Choi, Y. J. Chen and J. Szustakowki (2001). "Initial sequencing and analysis of the human genome." Nature 409(6822): 860-921.

Landgraf, S., J. Steingen, Y. Eppert, U. Niedermeyer, E. van der Meer and F. Krueger (2011). "Temporal information processing in short- and long-term memory of patients with schizophrenia." PLoS One 6(10): e26140.

Landré, L., C. Destrieux, F. Andersson, L. Barantin, Y. Quidé, G. Tapia, N. Jaafari, D. Clarys, P. Gaillard, M. Isingrini and W. El-Hage (2012). "Working memory processing of traumatic material in women with posttraumatic stress disorder." Journal of Psychiatry \& Neuroscience : JPN 37(2): 87-94.

Lasagna-Reeves, C. A., D. L. Castillo-Carranza, U. Sengupta, A. L. Clos, G. R. Jackson and R. Kayed (2011). "Tau oligomers impair memory and induce synaptic and mitochondrial dysfunction in wild-type mice." Mol Neurodegener 6: 39.

Lauritzen, S. P., T. L. Boye and J. Nylandsted (2015). "Annexins are instrumental for efficient plasma membrane repair in cancer cells." Semin Cell Dev Biol 45: 32-38. 
Lee, I. and R. P. Kesner (2003). "Time-dependent relationship between the dorsal hippocampus and the prefrontal cortex in spatial memory." J Neurosci 23(4): 1517-1523.

Lee, T. I. and R. A. Young (2000). "Transcription of eukaryotic protein-coding genes." Annu Rev Genet 34: 77-137.

Levenson, J. M., K. J. O'Riordan, K. D. Brown, M. A. Trinh, D. L. Molfese and J. D. Sweatt (2004). "Regulation of histone acetylation during memory formation in the hippocampus." J Biol Chem 279(39): 40545-40559.

Levin, J., F. Schmidt, C. Boehm, C. Prix, K. Botzel, S. Ryazanov, A. Leonov, C. Griesinger and A. Giese (2014). "The oligomer modulator anle138b inhibits disease progression in a Parkinson mouse model even with treatment started after disease onset." Acta Neuropathol 127(5): 779-780.

Lewandowsky, S., M. Duncan and G. D. Brown (2004). "Time does not cause forgetting in shortterm serial recall." Psychon Bull Rev 11(5): 771-790.

Li, J., J. Ma, G. Meng, H. Lin, S. Wu, J. Wang, J. Luo, X. Xu, D. Tough, M. Lindon, I. Rioja, J. Zhao, H. Mei, R. Prinjha and Z. Zhong (2016). "BET bromodomain inhibition promotes neurogenesis while inhibiting gliogenesis in neural progenitor cells." Stem Cell Res 17(2): 212-221.

Lin, M. T. and M. F. Beal (2006). "Mitochondrial dysfunction and oxidative stress in neurodegenerative diseases." Nature 443: 787.

Ling, Q., A. T. Jacovina, A. Deora, M. Febbraio, R. Simantov, R. L. Silverstein, B. Hempstead, W. H. Mark and K. A. Hajjar (2004). "Annexin II regulates fibrin homeostasis and neoangiogenesis in vivo." J Clin Invest 113(1): 38-48.

Ling, Q., A. T. Jacovina, A. Deora, M. Febbraio, R. Simantov, R. L. Silverstein, B. Hempstead, W. H. Mark and K. A. Hajjar (2004). "Annexin II regulates fibrin homeostasis and neoangiogenesis in vivo." Journal of Clinical Investigation 113(1): 38-48.

Liu, J., C. A. Rothermund, J. Ayala-Sanmartin and J. K. Vishwanatha (2003). "Nuclear annexin II negatively regulates growth of $\mathrm{LNCaP}$ cells and substitution of ser 11 and 25 to glu prevents nucleo-cytoplasmic shuttling of annexin II." BMC Biochem 4: 10.

Liu, J. and J. K. Vishwanatha (2007). "Regulation of nucleo-cytoplasmic shuttling of human annexin A2: a proposed mechanism." Mol Cell Biochem 303(1-2): 211-220.

Liu, L., V. Drouet, J. W. Wu, M. P. Witter, S. A. Small, C. Clelland and K. Duff (2012). "Transsynaptic spread of tau pathology in vivo." PLoS One 7.

Liu, L., A. B. Fisher and U. J. Zimmerman (1995). "Lung annexin II promotes fusion of isolated lamellar bodies with liposomes." Biochim Biophys Acta 1259(2): 166-172.

Liu, Y., H. K. Myrvang and L. V. Dekker (2015). "Annexin A2 complexes with S100 proteins: structure, function and pharmacological manipulation." $\mathrm{Br} J$ Pharmacol 172(7): 1664-1676.

Lonze, B. E. and D. D. Ginty (2002). "Function and regulation of CREB family transcription factors in the nervous system." Neuron 35(4): 605-623.

Lopez Sanchez, M. I. G., H. S. Waugh, A. Tsatsanis, B. X. Wong, J. G. Crowston, J. A. Duce and I. A. Trounce (2017). "Amyloid precursor protein drives down-regulation of mitochondrial oxidative phosphorylation independent of amyloid beta." Sci Rep 7(1): 9835. 
Lott, I. T. and E. Head (2005). "Alzheimer disease and Down syndrome: factors in pathogenesis." Neurobiol Aging 26(3): 383-389.

Lubin, F. D., S. Gupta, R. R. Parrish, N. M. Grissom and R. L. Davis (2011). "Epigenetic mechanisms: critical contributors to long-term memory formation." Neuroscientist 17(6): 616-632.

Luchsinger, J. A., M. X. Tang, S. Shea and R. Mayeux (2002). "Caloric intake and the risk of Alzheimer disease." Arch Neurol 59(8): 1258-1263.

Lum, J. A. G., G. Conti-Ramsden, D. Page and M. T. Ullman (2012). "Working, declarative and procedural memory in specific language impairment." Cortex; a Journal Devoted to the Study of the Nervous System and Behavior 48(9): 1138-1154.

Luo, W., G. Yan, L. Li, Z. Wang, H. Liu, S. Zhou, S. Liu, M. Tang, W. Yi, Z. Dong and Y. Cao (2008). "Epstein-Barr virus latent membrane protein 1 mediates serine 25 phosphorylation and nuclear entry of annexin A2 via PI-PLC-PKCalpha/PKCbeta pathway." Mol Carcinog 47(12): 934-946.

Luo, X. and W. L. Kraus (2012). "On PAR with PARP: cellular stress signaling through poly(ADP-ribose) and PARP-1." Genes Dev 26(5): 417-432.

MacDuffie, K. E., A. S. Atkins, K. E. Flegal, C. M. Clark and P. A. Reuter-Lorenz (2012). "Memory Distortion in Alzheimer's Disease: Deficient Monitoring of Short and Longterm Memory." Neuropsychology 26(4): 509-516.

Madabhushi, R., F. Gao, A. R. Pfenning, L. Pan, S. Yamakawa, J. Seo, R. Rueda, T. X. Phan, H. Yamakawa, P. C. Pao, R. T. Stott, E. Gjoneska, A. Nott, S. Cho, M. Kellis and L. H. Tsai (2015). "Activity-Induced DNA Breaks Govern the Expression of Neuronal Early-Response Genes." Cell 161(7): 1592-1605.

Madureira, P. A., R. Hill, P. W. Lee and D. M. Waisman (2012). "Genotoxic agents promote the nuclear accumulation of annexin A2: role of annexin A2 in mitigating DNA damage." PLoS One 7(11): e50591.

Magistri, M., D. Velmeshev, M. Makhmutova, P. Patel, G. C. Sartor, C.-H. Volmar, C. Wahlestedt and M. A. Faghihi (2016). "The BET-Bromodomain Inhibitor JQ1 Reduces Inflammation and Tau Phosphorylation at Ser396 in the Brain of the 3xTg Model of Alzheimer's Disease." Current Alzheimer Research 13(9): 985-995.

Mahut, H., S. Zola-Morgan and M. Moss (1982). "Hippocampal resections impair associative learning and recognition memory in the monkey." J Neurosci 2(9): 1214-1220.

Maji, S., P. Chaudhary, I. Akopova, P. M. Nguyen, R. J. Hare, I. Gryczynski and J. K. Vishwanatha (2017). "Exosomal Annexin II Promotes Angiogenesis and Breast Cancer Metastasis." Mol Cancer Res 15(1): 93-105.

Martin, K. C., A. Casadio, H. Zhu, E. Yaping, J. C. Rose, M. Chen, C. H. Bailey and E. R. Kandel (1997). "Synapse-specific, long-term facilitation of aplysia sensory to motor synapses: a function for local protein synthesis in memory storage." Cell 91(7): 927-938.

Masters, C. L., G. Simms, N. A. Weinman, G. Multhaup, B. L. McDonald and K. Beyreuther (1985). "Amyloid plaque core protein in Alzheimer disease and Down syndrome." Proc Natl Acad Sci U S A 82(12): 4245-4249.

Mattson, M. P. (2004). "Pathways towards and away from Alzheimer's disease." Nature 430(7000): 631-639. 
Mawuenyega, K. G., T. Kasten, W. Sigurdson and R. J. Bateman (2013). "Amyloid-beta Isoform Metabolism Quantitation by Stable Isotope Labeled Kinetics." Analytical biochemistry 440(1): 56-62.

McClure, C., K. L. Cole, P. Wulff, M. Klugmann and A. J. Murray (2011). "Production and titering of recombinant adeno-associated viral vectors." J Vis Exp(57): e3348.

Mcllwain, K. L., M. Y. Merriweather, L. A. Yuva-Paylor and R. Paylor (2001). "The use of behavioral test batteries: effects of training history." Physiol Behav 73(5): 705-717.

Mickleburgh, I., B. Burtle, H. Hollas, G. Campbell, Z. Chrzanowska-Lightowlers, A. Vedeler and J. Hesketh (2005). "Annexin A2 binds to the localization signal in the 3 ' untranslated region of c-myc mRNA." Febs j 272(2): 413-421.

Mietzsch, M., F. Broecker, A. Reinhardt, P. H. Seeberger and R. Heilbronn (2014). "Differential adeno-associated virus serotype-specific interaction patterns with synthetic heparins and other glycans." J Virol 88(5): 2991-3003.

Miller, C. A., S. L. Campbell and J. D. Sweatt (2008). "DNA methylation and histone acetylation work in concert to regulate memory formation and synaptic plasticity." Neurobiol Learn Mem 89(4): 599-603.

Miller, G. A. (1956). "The magical number seven plus or minus two: some limits on our capacity for processing information." Psychol Rev 63(2): 81-97.

Mills, J. D., T. Nalpathamkalam, H. I. Jacobs, C. Janitz, D. Merico, P. Hu and M. Janitz (2013). "RNA-Seq analysis of the parietal cortex in Alzheimer's disease reveals alternatively spliced isoforms related to lipid metabolism." Neurosci Lett 536: 90-95.

Milward, E. A., R. Papadopoulos, S. J. Fuller, R. D. Moir, D. Small, K. Beyreuther and C. L. Masters (1992). "The amyloid protein precursor of Alzheimer's disease is a mediator of the effects of nerve growth factor on neurite outgrowth." Neuron 9(1): 129-137.

Min, S. W., X. Chen, T. E. Tracy, Y. Li, Y. Zhou, C. Wang, K. Shirakawa, S. S. Minami, E. Defensor, S. A. Mok, P. D. Sohn, B. Schilling, X. Cong, L. Ellerby, B. W. Gibson, J. Johnson, N. Krogan, M. Shamloo, J. Gestwicki, E. Masliah, E. Verdin and L. Gan (2015). "Critical role of acetylation in tau-mediated neurodegeneration and cognitive deficits." Nat Med 21(10): 1154-1162.

Min, S. W., S. H. Cho, Y. Zhou, S. Schroeder, V. Haroutunian, W. W. Seeley, E. J. Huang, Y. Shen, E. Masliah, C. Mukherjee, D. Meyers, P. A. Cole, M. Ott and L. Gan (2010). "Acetylation of tau inhibits its degradation and contributes to tauopathy." Neuron 67.

Ming, G. L. and H. Song (2005). "Adult neurogenesis in the mammalian central nervous system." Annu Rev Neurosci 28: 223-250.

Molfese, D. L. (2011). "Advancing neuroscience through epigenetics: molecular mechanisms of learning and memory." Dev Neuropsychol 36(7): 810-827.

Mongillo, G., O. Barak and M. Tsodyks (2008). "Synaptic theory of working memory." Science 319(5869): 1543-1546.

Montminy, M. R. and L. M. Bilezikjian (1987). "Binding of a nuclear protein to the cyclic-AMP response element of the somatostatin gene." Nature 328(6126): 175-178.

Montminy, M. R., M. J. Low, L. Tapia-Arancibia, S. Reichlin, G. Mandel and R. H. Goodman (1986). "Cyclic AMP regulates somatostatin mRNA accumulation in primary 
diencephalic cultures and in transfected fibroblast cells." J Neurosci 6(4): 1171-1176.

Mootha, V. K., C. M. Lindgren, K. F. Eriksson, A. Subramanian, S. Sihag, J. Lehar, P. Puigserver, E. Carlsson, M. Ridderstrale, E. Laurila, N. Houstis, M. J. Daly, N. Patterson, J. P. Mesirov, T. R. Golub, P. Tamayo, B. Spiegelman, E. S. Lander, J. N. Hirschhorn, D. Altshuler and L. C. Groop (2003). "PGC-1alpha-responsive genes involved in oxidative phosphorylation are coordinately downregulated in human diabetes." Nat Genet 34(3): 267-273.

Morales, V. and H. Richard-Foy (2000). "Role of Histone N-Terminal Tails and Their Acetylation in Nucleosome Dynamics." Molecular and Cellular Biology 20(19): 7230-7237.

Moriniere, J., S. Rousseaux, U. Steuerwald, M. Soler-Lopez, S. Curtet, A. L. Vitte, J. Govin, J. Gaucher, K. Sadoul, D. J. Hart, J. Krijgsveld, S. Khochbin, C. W. Muller and C. Petosa (2009). "Cooperative binding of two acetylation marks on a histone tail by a single bromodomain." Nature 461(7264): 664-668.

Morris, M., G. M. Knudsen, S. Maeda, J. C. Trinidad, A. loanoviciu, A. L. Burlingame and L. Mucke (2015). "Tau post-translational modifications in wild-type and human amyloid precursor protein transgenic mice." Nat Neurosci 18(8): 1183-1189.

Morris, R. G. M. (1981). "Spatial localization does not require the presence of local cues." Learning and Motivation 12(2): 239-260.

Moser, E., M. B. Moser and P. Andersen (1993). "Spatial learning impairment parallels the magnitude of dorsal hippocampal lesions, but is hardly present following ventral lesions." J Neurosci 13(9): 3916-3925.

Mosmann, T. (1983). "Rapid colorimetric assay for cellular growth and survival: application to proliferation and cytotoxicity assays." J Immunol Methods 65(1-2): 55-63.

Moss, S. E. and R. O. Morgan (2004). "The annexins." Genome Biol 5(4): 219.

Mussunoor, S. and G. I. Murray (2008). "The role of annexins in tumour development and progression." J Pathol 216(2): 131-140.

Nazmi, A. R., G. Ozorowski, M. Pejic, J. P. Whitelegge, V. Gerke and H. Luecke (2012). "Nterminal acetylation of annexin $\mathrm{A} 2$ is required for S100A10 binding." Biol Chem 393(10): 1141-1150.

Noack, M., J. Leyk and C. Richter-Landsberg (2014). "HDAC6 inhibition results in tau acetylation and modulates tau phosphorylation and degradation in oligodendrocytes." Glia 62(4): 535-547.

Oh, Y. S., P. Gao, K. W. Lee, I. Ceglia, J. S. Seo, X. Zhang, J. H. Ahn, B. T. Chait, D. J. Patel, Y. Kim and P. Greengard (2013). "SMARCA3, a chromatin-remodeling factor, is required for p11-dependent antidepressant action." Cell 152(4): 831-843.

Panza, F., V. Frisardi, V. Solfrizzi, B. P. Imbimbo, G. Logroscino, A. Santamato, A. Greco, D. Seripa and A. Pilotto (2012). "Immunotherapy for Alzheimer's disease: from antibeta-amyloid to tau-based immunization strategies." Immunotherapy 4(2): 213-238.

Parker, E. S., L. Cahill and J. L. McGaugh (2006). "A case of unusual autobiographical remembering." Neurocase 12(1): 35-49.

Pavlov, P. I. (2010). "Conditioned reflexes: An investigation of the physiological activity of the cerebral cortex." Annals of Neurosciences 17(3): 136-141. 
Peleg, S., F. Sananbenesi, A. Zovoilis, S. Burkhardt, S. Bahari-Javan, R. C. Agis-Balboa, P. Cota, J. L. Wittnam, A. Gogol-Doering, L. Opitz, G. Salinas-Riester, M. Dettenhofer, H. Kang, L. Farinelli, W. Chen and A. Fischer (2010). "Altered histone acetylation is associated with age-dependent memory impairment in mice." Science 328(5979): 753-756.

Pellow, S., P. Chopin, S. E. File and M. Briley (1985). "Validation of open : closed arm entries in an elevated plus-maze as a measure of anxiety in the rat." Journal of Neuroscience Methods 14(3): 149-167.

Peterson, L. R. and M. J. Peterson (1959). "Short-term retention of individual verbal items." $\underline{J}$ Exp Psychol 58: 193-198.

Pittenger, C., Y. Y. Huang, R. F. Paletzki, R. Bourtchouladze, H. Scanlin, S. Vronskaya and E. R. Kandel (2002). "Reversible inhibition of CREB/ATF transcription factors in region CA1 of the dorsal hippocampus disrupts hippocampus-dependent spatial memory." Neuron 34(3): 447-462.

Plagg, B., D. Ehrlich, K. M. Kniewallner, J. Marksteiner and C. Humpel (2015). "Increased Acetylation of Histone H4 at Lysine 12 (H4K12) in Monocytes of Transgenic Alzheimer's Mice and in Human Patients." Current Alzheimer research 12(8): 752-760.

Polster, M. R., L. Nadel and D. L. Schacter (1991). "Cognitive neuroscience analyses of memory: a historical perspective." J Cogn Neurosci 3(2): 95-116.

Porsolt, R. D., M. Le Pichon and M. Jalfre (1977). "Depression: a new animal model sensitive to antidepressant treatments." Nature 266(5604): 730-732.

Portelius, E., G. Brinkmalm, A. J. Tran, H. Zetterberg, A. Westman-Brinkmalm and K. Blennow (2009). "Identification of novel APP/Abeta isoforms in human cerebrospinal fluid." Neurodegener Dis 6(3): 87-94.

Price, J. L. and J. C. Morris (1999). "Tangles and plaques in nondemented aging and "preclinical" Alzheimer's disease." Ann Neurol 45(3): 358-368.

Priller, C., T. Bauer, G. Mitteregger, B. Krebs, H. A. Kretzschmar and J. Herms (2006). "Synapse formation and function is modulated by the amyloid precursor protein." $\mathrm{J}$ Neurosci 26(27): 7212-7221.

Qiu, H., A. L. Jackson, J. E. Kilgore, Y. Zhong, L. L. Chan, P. A. Gehrig, C. Zhou and V. L. BaeJump (2015). "JQ1 suppresses tumor growth through downregulating LDHA in ovarian cancer." Oncotarget 6(9): 6915-6930.

Qiu, W. Q., A. Ferreira, C. Miller, E. H. Koo and D. J. Selkoe (1995). "Cell-surface beta-amyloid precursor protein stimulates neurite outgrowth of hippocampal neurons in an isoform-dependent manner." J Neurosci 15(3 Pt 2): 2157-2167.

Rapti, K., V. Louis-Jeune, E. Kohlbrenner, K. Ishikawa, D. Ladage, S. Zolotukhin, R. J. Hajjar and T. Weber (2012). "Neutralizing antibodies against AAV serotypes 1, 2, 6, and 9 in sera of commonly used animal models." Mol Ther 20(1): 73-83.

Raynal, P. and H. B. Pollard (1994). "Annexins: the problem of assessing the biological role for a gene family of multifunctional calcium- and phospholipid-binding proteins." Biochim Biophys Acta 1197(1): 63-93.

Reeves, E. K., H. Gordish-Dressman, E. P. Hoffman and Y. Hathout (2009). "Proteomic profiling of glucocorticoid-exposed myogenic cells: Time series assessment of protein translocation and transcription of inactive mRNAs." Proteome Sci 7: 26. 
Rintala-Dempsey, A. C., A. Rezvanpour and G. S. Shaw (2008). "S100-annexin complexes-structural insights." Febs j 275(20): 4956-4966.

Risso, D., J. Ngai, T. P. Speed and S. Dudoit (2014). "Normalization of RNA-seq data using factor analysis of control genes or samples." Nat Biotech 32(9): 896-902.

Roberson, E. D., B. Halabisky, J. W. Yoo, J. Yao, J. Chin, F. Yan, T. Wu, P. Hamto, N. Devidze, G. Q. Yu, J. J. Palop, J. L. Noebels and L. Mucke (2011). "Amyloid-beta/Fyninduced synaptic, network, and cognitive impairments depend on tau levels in multiple mouse models of Alzheimer's disease." J Neurosci 31(2): 700-711.

Rodriguez, F., J. C. Lopez, J. P. Vargas, C. Broglio, Y. Gomez and C. Salas (2002). "Spatial memory and hippocampal pallium through vertebrate evolution: insights from reptiles and teleost fish." Brain Res Bull 57(3-4): 499-503.

Rojas-Duran, M. F. and W. V. Gilbert (2012). "Alternative transcription start site selection leads to large differences in translation activity in yeast." RNA 18(12): 2299-2305.

Rousseaux, S. and S. Khochbin (2015). "Histone Acylation beyond Acetylation: Terra Incognita in Chromatin Biology." Cell J 17(1): 1-6.

Rudy, J. W., N. C. Huff and P. Matus-Amat (2004). "Understanding contextual fear conditioning: insights from a two-process model." Neurosci Biobehav Rev 28(7): 675-685.

Sakabe, K., Z. Wang and G. W. Hart (2010). "Beta-N-acetylglucosamine (O-GIcNAc) is part of the histone code." Proc Natl Acad Sci U S A 107(46): 19915-19920.

Sarkar, S., R. Swiercz, C. Kantara, K. A. Hajjar and P. Singh (2011). "Annexin A2 mediates upregulation of NF-kappaB, beta-catenin, and stem cell in response to progastrin in mice and HEK-293 cells." Gastroenterology 140(2): 583-595.e584.

Sartor, G. C., S. K. Powell, S. P. Brothers and C. Wahlestedt (2015). "Epigenetic Readers of Lysine Acetylation Regulate Cocaine-Induced Plasticity." J Neurosci 35(45): 15062-15072.

Saunders, R. C. and D. L. Rosene (1988). "A comparison of the efferents of the amygdala and the hippocampal formation in the rhesus monkey: I. Convergence in the entorhinal, prorhinal, and perirhinal cortices." J Comp Neurol 271(2): 153-184.

Schafe, G. E., K. Nader, H. T. Blair and J. E. LeDoux (2001). "Memory consolidation of Pavlovian fear conditioning: a cellular and molecular perspective." Trends Neurosci 24(9): 540-546.

Schafer, G., J. K. Hitchcock, T. M. Shaw, A. A. Katz and M. I. Parker (2015). "A novel role of annexin A2 in human type I collagen gene expression." J Cell Biochem 116(3): 408-417.

Scharfman, H. E. (2007). "The CA3 "Backprojection" to the Dentate Gyrus." Progress in brain research 163: 627-637.

Schindowski, K., A. Bretteville, K. Leroy, S. Begard, J. P. Brion, M. Hamdane and L. Buee (2006). "Alzheimer's disease-like tau neuropathology leads to memory deficits and loss of functional synapses in a novel mutated tau transgenic mouse without any motor deficits." Am J Pathol 169(2): 599-616.

Schmidt, M., A. Voutetakis, S. Afione, C. Zheng, D. Mandikian and J. A. Chiorini (2008). "Adenoassociated virus type 12 (AAV12): a novel AAV serotype with sialic acid- and

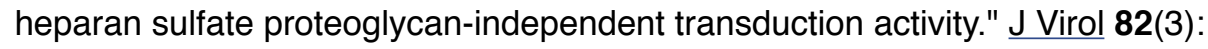
1399-1406. 
Schoenenberger, P., D. Gerosa and T. G. Oertner (2009). "Temporal Control of Immediate Early Gene Induction by Light." PLoS One 4(12).

Schor, I. E. and A. R. Kornblihtt (2009). "Playing inside the genes: Intragenic histone acetylation after membrane depolarization of neural cells opens a path for alternative splicing regulation." Communicative \& Integrative Biology 2(4): 341-343.

Scoville, W. B. and B. Milner (1957). "Loss of recent memory after bilateral hippocampal lesions." J Neurol Neurosurg Psychiatry 20(1): 11-21.

Selenica, M. L., L. Benner, S. B. Housley, B. Manchec, D. C. Lee, K. R. Nash, J. Kalin, J. A. Bergman, A. Kozikowski, M. N. Gordon and D. Morgan (2014). "Histone deacetylase 6 inhibition improves memory and reduces total tau levels in a mouse model of tau deposition." Alzheimers Res Ther 6(1): 12.

Selkoe, D. J. and J. Hardy (2016). "The amyloid hypothesis of Alzheimer's disease at 25 years." EMBO Mol Med 8(6): 595-608.

Senechal, Y., P. H. Kelly and K. K. Dev (2008). "Amyloid precursor protein knockout mice show age-dependent deficits in passive avoidance learning." Behav Brain Res 186(1): 126-132.

Senkov, O., P. Andjus, L. Radenovic, E. Soriano and A. Dityatev (2014). "Neural ECM molecules in synaptic plasticity, learning, and memory." Prog Brain Res 214: 53-80.

Serrano-Pozo, A., M. P. Frosch, E. Masliah and B. T. Hyman (2011). "Neuropathological Alterations in Alzheimer Disease." Cold Spring Harbor Perspectives in Medicine: 1(1): a006189.

Seto, E. and M. Yoshida (2014). "Erasers of Histone Acetylation: The Histone Deacetylase Enzymes." Cold Spring Harbor Perspectives in Biology 6(4): a018713.

Sewal, A. S., H. Patzke, E. J. Perez, P. Park, E. Lehrmann, Y. Zhang, K. G. Becker, B. R. Fletcher, J. M. Long and P. R. Rapp (2015). "Experience Modulates the Effects of Histone Deacetylase Inhibitors on Gene and Protein Expression in the Hippocampus: Impaired Plasticity in Aging." J Neurosci 35(33): 11729-11742.

Shadmehr, R. and T. Brashers-Krug (1997). "Functional Stages in the Formation of Human Long-Term Motor Memory." The Journal of Neuroscience 17(1): 409.

Sharma, M. C. and M. Sharma (2007). "The role of annexin II in angiogenesis and tumor progression: a potential therapeutic target." Curr Pharm Des 13(35): 3568-3575.

Shiio, Y. and R. N. Eisenman (2003). "Histone sumoylation is associated with transcriptional repression." Proc Natl Acad Sci U S A 100(23): 13225-13230.

Shin, R.-W., K. Ogino, A. Kondo, T. C. Saido, J. Q. Trojanowski, T. Kitamoto and J. Tateishi (1997). "Amyloid $\beta$-Protein $(A \beta)$ 1-40 But Not A $\beta 1-42$ Contributes to the Experimental Formation of Alzheimer Disease Amyloid Fibrils in Rat Brain." The Journal of Neuroscience 17(21): 8187-8193.

Spellman, D. S., K. Deinhardt, C. C. Darie, M. V. Chao and T. A. Neubert (2008). "Stable isotopic labeling by amino acids in cultured primary neurons: application to brainderived neurotrophic factor-dependent phosphotyrosine-associated signaling." Mol Cell Proteomics 7(6): 1067-1076.

Spires-Jones, T. L., K. J. Kopeikina, R. M. Koffie, A. de Calignon and B. T. Hyman (2011). "Are tangles as toxic as they look?" J Mol Neurosci 45(3): 438-444. 
Star, E. N., D. J. Kwiatkowski and V. N. Murthy (2002). "Rapid turnover of actin in dendritic spines and its regulation by activity." Nat Neurosci 5(3): 239-246.

Stefanko, D. P., R. M. Barrett, A. R. Ly, G. K. Reolon and M. A. Wood (2009). "Modulation of long-term memory for object recognition via HDAC inhibition." Proc Natl Acad Sci U S A 106(23): 9447-9452.

Strahl, B. D. and C. D. Allis (2000). "The language of covalent histone modifications." Nature 403(6765): 41-45.

Strosznajder, R. P., H. Jesko and A. Adamczyk (2005). "Effect of aging and oxidative/genotoxic stress on poly(ADP-ribose) polymerase-1 activity in rat brain." Acta Biochim Pol 52(4): 909-914.

Stucki, M., J. A. Clapperton, D. Mohammad, M. B. Yaffe, S. J. Smerdon and S. P. Jackson (2005). "MDC1 directly binds phosphorylated histone H2AX to regulate cellular responses to DNA double-strand breaks." Cell 123(7): 1213-1226.

Subramanian, A., P. Tamayo, V. K. Mootha, S. Mukherjee, B. L. Ebert, M. A. Gillette, A. Paulovich, S. L. Pomeroy, T. R. Golub, E. S. Lander and J. P. Mesirov (2005). "Gene set enrichment analysis: a knowledge-based approach for interpreting genome-wide expression profiles." Proc Natl Acad Sci U S A 102(43): 15545-15550.

Suh, Y. H., A. Terashima, R. S. Petralia, R. J. Wenthold, J. T. R. Isaac, K. W. Roche and P. A. Roche (2010). "A neuronal role for SNAP-23 in postsynaptic glutamate receptor trafficking." Nature neuroscience 13(3): 338-343.

Summerford, C. and R. J. Samulski (1998). "Membrane-associated heparan sulfate proteoglycan is a receptor for adeno-associated virus type 2 virions." $\mathrm{J}$ Virol 72(2): 1438-1445.

Sutherland, R. J., J. O'Brien and H. Lehmann (2008). "Absence of systems consolidation of fear memories after dorsal, ventral, or complete hippocampal damage." Hippocampus 18(7): $710-718$.

Sutherland, R. J. and J. W. Rudy (1988). "Place learning in the Morris place navigation task is impaired by damage to the hippocampal formation even if the temporal demands are reduced." Psychobiology 16(2): 157-163.

Tan, M., H. Luo, S. Lee, F. Jin, J. S. Yang, E. Montellier, T. Buchou, Z. Cheng, S. Rousseaux, N. Rajagopal, Z. Lu, Z. Ye, Q. Zhu, J. Wysocka, Y. Ye, S. Khochbin, B. Ren and Y. Zhao (2011). "Identification of 67 histone marks and histone lysine crotonylation as a new type of histone modification." Cell 146(6): 1016-1028.

Taverna, S. D., H. Li, A. J. Ruthenburg, C. D. Allis and D. J. Patel (2007). "How chromatinbinding modules interpret histone modifications: lessons from professional pocket pickers." Nat Struct Mol Biol 14(11): 1025-1040.

Terni, B. and I. Ferrer (2015). "Abnormal Expression and Distribution of MMP2 at Initial Stages of Alzheimer's Disease-Related Pathology." J Alzheimers Dis 46(2): 461-469.

Thierry, A. M., Y. Gioanni, E. Degenetais and J. Glowinski (2000). "Hippocampo-prefrontal cortex pathway: anatomical and electrophysiological characteristics." Hippocampus 10(4): 411-419.

Thornton, A. E. and N. Raz (1997). "Memory impairment in multiple sclerosis: a quantitative review." Neuropsychology 11(3): 357-366. 
Tomas, A. and S. E. Moss (2003). "Calcium- and cell cycle-dependent association of annexin 11 with the nuclear envelope." J Biol Chem 278(22): 20210-20216.

Tomlinson, B. E., G. Blessed and M. Roth (1970). "Observations on the brains of demented old people." Journal of the Neurological Sciences 11(3): 205-242.

Trudeau, L. E. and V. F. Castellucci (1993). "Excitatory amino acid neurotransmission at sensory-motor and interneuronal synapses of Aplysia californica." J Neurophysiol 70(3): 1221-1230.

Tseng, J.-H., L. Xie, S. Song, Y. Xie, L. Allen, D. Ajit, J.-S. Hong, X. Chen, R. B. Meeker and T. J. Cohen (2017). "The Deacetylase HDAC6 Mediates Endogenous Neuritic Tau Pathology." Cell reports 20(9): 2169-2183.

Tsilibary, E., A. Tzinia, L. Radenovic, V. Stamenkovic, T. Lebitko, M. Mucha, R. Pawlak, R. Frischknecht and L. Kaczmarek (2014). Chapter 6 - Neural ECM proteases in learning and synaptic plasticity. Progress in Brain Research. A. Dityatev, B. WehrleHaller and A. Pitkänen, Elsevier. 214: 135-157.

Tyan, S. H., A. Y. Shih, J. J. Walsh, H. Maruyama, F. Sarsoza, L. Ku, S. Eggert, P. R. Hof, E. H. Koo and D. L. Dickstein (2012). "Amyloid precursor protein (APP) regulates synaptic structure and function." Mol Cell Neurosci 51(1-2): 43-52.

Umehara, T., Y. Nakamura, M. K. Jang, K. Nakano, A. Tanaka, K. Ozato, B. Padmanabhan and S. Yokoyama (2010). "Structural basis for acetylated histone $\mathrm{H} 4$ recognition by the human BRD2 bromodomain." J Biol Chem 285(10): 7610-7618.

Valapala, M., S. Maji, J. Borejdo and J. K. Vishwanatha (2014). "Cell Surface Translocation of Annexin A2 Facilitates Glutamate-induced Extracellular Proteolysis." The Journal of Biological Chemistry 289(23): 15915-15926.

Van der Jeugd, A., D. Blum, S. Raison, S. Eddarkaoui, L. Buee and R. D'Hooge (2013). "Observations in THY-Tau22 mice that resemble behavioral and psychological signs and symptoms of dementia." Behav Brain Res 242: 34-39.

Vassar, R., P. H. Kuhn, C. Haass, M. E. Kennedy, L. Rajendran, P. C. Wong and S. F. Lichtenthaler (2014). "Function, therapeutic potential and cell biology of BACE proteases: current status and future prospects." J Neurochem 130(1): 4-28.

Vasudevan, A., M. S. Ho, M. Weiergraber, R. Nischt, T. Schneider, A. Lie, N. Smyth and R. Kohling (2010). "Basement membrane protein nidogen-1 shapes hippocampal synaptic plasticity and excitability." Hippocampus 20(5): 608-620.

Vedeler, A. and H. Hollas (2000). "Annexin II is associated with mRNAs which may constitute a distinct subpopulation." Biochem J 348 Pt 3: 565-572.

Vedeler, A., H. Hollas, A. K. Grindheim and A. M. Raddum (2012). "Multiple roles of annexin A2 in post-transcriptional regulation of gene expression." Curr Protein Pept Sci 13(4): 401-412.

Venter, J. C., M. D. Adams, E. W. Myers, P. W. Li, R. J. Mural, G. G. Sutton, H. O. Smith, M. Yandell, C. A. Evans, R. A. Holt, J. D. Gocayne, P. Amanatides, R. M. Ballew, D. H. Huson, J. R. Wortman, Q. Zhang, C. D. Kodira, X. H. Zheng, L. Chen, M. Skupski, G. Subramanian, P. D. Thomas, J. Zhang, G. L. Gabor Miklos, C. Nelson, S. Broder, A. G. Clark, J. Nadeau, V. A. McKusick, N. Zinder, A. J. Levine, R. J. Roberts, M. Simon, C. Slayman, M. Hunkapiller, R. Bolanos, A. Delcher, I. Dew, D. Fasulo, M. Flanigan, L. Florea, A. Halpern, S. Hannenhalli, S. Kravitz, S. Levy, C. Mobarry, K. Reinert, K. Remington, J. Abu-Threideh, E. Beasley, K. Biddick, V. Bonazzi, R. Brandon, M. Cargill, I. Chandramouliswaran, R. Charlab, K. 
Chaturvedi, Z. Deng, V. Di Francesco, P. Dunn, K. Eilbeck, C. Evangelista, A. E. Gabrielian, W. Gan, W. Ge, F. Gong, Z. Gu, P. Guan, T. J. Heiman, M. E. Higgins, R. R. Ji, Z. Ke, K. A. Ketchum, Z. Lai, Y. Lei, Z. Li, J. Li, Y. Liang, X. Lin, F. Lu, G. V. Merkulov, N. Milshina, H. M. Moore, A. K. Naik, V. A. Narayan, B. Neelam, D. Nusskern, D. B. Rusch, S. Salzberg, W. Shao, B. Shue, J. Sun, Z. Wang, A. Wang, X. Wang, J. Wang, M. Wei, R. Wides, C. Xiao, C. Yan, A. Yao, J. Ye, M. Zhan, W. Zhang, H. Zhang, Q. Zhao, L. Zheng, F. Zhong, W. Zhong, S. Zhu, S. Zhao, D. Gilbert, S. Baumhueter, G. Spier, C. Carter, A. Cravchik, T. Woodage, F. Ali, H. An, A. Awe, D. Baldwin, H. Baden, M. Barnstead, I. Barrow, K. Beeson, D. Busam, A. Carver, A. Center, M. L. Cheng, L. Curry, S. Danaher, L. Davenport, R. Desilets, S. Dietz, K. Dodson, L. Doup, S. Ferriera, N. Garg, A. Gluecksmann, B. Hart, J. Haynes, C. Haynes, C. Heiner, S. Hladun, D. Hostin, J. Houck, T. Howland, C. Ibegwam, J. Johnson, F. Kalush, L. Kline, S. Koduru, A. Love, F. Mann, D. May, S. McCawley, T. McIntosh, I. McMullen, M. Moy, L. Moy, B. Murphy, K. Nelson, C. Pfannkoch, E. Pratts, V. Puri, H. Qureshi, M. Reardon, R. Rodriguez, Y. H. Rogers, D. Romblad, B. Ruhfel, R. Scott, C. Sitter, M. Smallwood, E. Stewart, R. Strong, E. Suh, R. Thomas, N. N. Tint, S. Tse, C. Vech, G. Wang, J. Wetter, S. Williams, M. Williams, S. Windsor, E. Winn-Deen, K. Wolfe, J. Zaveri, K. Zaveri, J. F. Abril, R. Guigo, M. J. Campbell, K. V. Sjolander, B. Karlak, A. Kejariwal, H. Mi, B. Lazareva, T. Hatton, A. Narechania, K. Diemer, A. Muruganujan, N. Guo, S. Sato, V. Bafna, S. Istrail, R. Lippert, R. Schwartz, B. Walenz, S. Yooseph, D. Allen, A. Basu, J. Baxendale, L. Blick, M. Caminha, J. Carnes-Stine, P. Caulk, Y. H. Chiang, M. Coyne, C. Dahlke, A. Mays, M. Dombroski, M. Donnelly, D. Ely, S. Esparham, C. Fosler, H. Gire, S. Glanowski, K. Glasser, A. Glodek, M. Gorokhov, K. Graham, B. Gropman, M. Harris, J. Heil, S. Henderson, J. Hoover, D. Jennings, C. Jordan, J. Jordan, J. Kasha, L. Kagan, C. Kraft, A. Levitsky, M. Lewis, X. Liu, J. Lopez, D. Ma, W. Majoros, J. McDaniel, S. Murphy, M. Newman, T. Nguyen, N. Nguyen, M. Nodell, S. Pan, J. Peck, M. Peterson, W. Rowe, R. Sanders, J. Scott, M. Simpson, T. Smith, A. Sprague, T. Stockwell, R. Turner, E. Venter, M. Wang, M. Wen, D. Wu, M. Wu, A. Xia, A. Zandieh and X. Zhu (2001). "The sequence of the human genome." Science 291(5507): 1304-1351.

Violet, M., L. Delattre, M. Tardivel, A. Sultan, A. Chauderlier, R. Caillierez, S. Talahari, F. Nesslany, B. Lefebvre, E. Bonnefoy, L. Buee and M. C. Galas (2014). "A major role for Tau in neuronal DNA and RNA protection in vivo under physiological and hyperthermic conditions." Front Cell Neurosci 8: 84.

Vishwanatha, J. K., H. K. Jindal and R. G. Davis (1992). "The role of primer recognition proteins in DNA replication: association with nuclear matrix in HeLa cells." J Cell Sci 101 (Pt 1): 25-34.

Vogel, F. (1964). "A PRELIMINARY ESTIMATE OF THE NUMBER OF HUMAN GENES." Nature 201: 847.

Vogelauer, M., J. Wu, N. Suka and M. Grunstein (2000). "Global histone acetylation and deacetylation in yeast." Nature 408(6811): 495-498.

Voronkov, M., S. P. Braithwaite and J. B. Stock (2011). "Phosphoprotein phosphatase 2A: a novel druggable target for Alzheimer's disease." Future Medicinal Chemistry 3(7): 821-833.

Wagner, J., S. Krauss, S. Shi, S. Ryazanov, J. Steffen, C. Miklitz, A. Leonov, A. Kleinknecht, B. Goricke, J. H. Weishaupt, D. Weckbecker, A. M. Reiner, W. Zinth, J. Levin, D. Ehninger, S. Remy, H. A. Kretzschmar, C. Griesinger, A. Giese and M. Fuhrmann 
(2015). "Reducing tau aggregates with anle138b delays disease progression in a mouse model of tauopathies." Acta Neuropathol 130(5): 619-631.

Wagner, J., S. Ryazanov, A. Leonov, J. Levin, S. Shi, F. Schmidt, C. Prix, F. Pan-Montojo, U. Bertsch, G. Mitteregger-Kretzschmar, M. Geissen, M. Eiden, F. Leidel, T. Hirschberger, A. A. Deeg, J. J. Krauth, W. Zinth, P. Tavan, J. Pilger, M.

Zweckstetter, T. Frank, M. Bahr, J. H. Weishaupt, M. Uhr, H. Urlaub, U. Teichmann, M. Samwer, K. Botzel, M. Groschup, H. Kretzschmar, C. Griesinger and A. Giese (2013). "Anle138b: a novel oligomer modulator for disease-modifying therapy of neurodegenerative diseases such as prion and Parkinson's disease." Acta Neuropathol 125(6): 795-813.

Waisman, D. M. (1995). "Annexin II tetramer: structure and function." Mol Cell Biochem 149-150: 301-322.

Wang, C. Y. and C. F. Lin (2014). "Annexin A2: its molecular regulation and cellular expression in cancer development." Dis Markers 2014: 308976.

Wang, P., N. R. Chintagari, D. Gou, L. Su and L. Liu (2007). "Physical and functional interactions of SNAP-23 with annexin A2." Am J Respir Cell Mol Biol 37(4): 467-476.

Watson, J. D. (2014). Molecular biology of the gene.

Wei, Y. H. and H. C. Lee (2002). "Oxidative stress, mitochondrial DNA mutation, and impairment of antioxidant enzymes in aging." Exp Biol Med (Maywood) 227(9): 671-682.

Weingarten, M. D., A. H. Lockwood, S. Y. Hwo and M. W. Kirschner (1975). "A protein factor essential for microtubule assembly." Proceedings of the National Academy of Sciences 72(5): 1858-1862.

Whishaw, I. Q. and J. Tomie (1996). "Of mice and mazes: similarities between mice and rats on dry land but not water mazes." Physiol Behav 60(5): 1191-1197.

White, R. J. and S. P. Jackson (1992). "The TATA-binding protein: a central role in transcription by RNA polymerases I, II and III." Trends Genet 8(8): 284-288.

Wiera, G., D. Nowak, I. van Hove, P. Dziegiel, L. Moons and J. W. Mozrzymas (2017). "Mechanisms of NMDA Receptor- and Voltage-Gated L-Type Calcium ChannelDependent Hippocampal LTP Critically Rely on Proteolysis That Is Mediated by Distinct Metalloproteinases." J Neurosci 37(5): 1240-1256.

Winocur, G., J. M. Wojtowicz, M. Sekeres, J. S. Snyder and S. Wang (2006). "Inhibition of neurogenesis interferes with hippocampus-dependent memory function." Hippocampus 16(3): 296-304.

Wirths, O., G. Multhaup, C. Czech, V. Blanchard, S. Moussaoui, G. Tremp, L. Pradier, K. Beyreuther and T. A. Bayer (2001). "Intraneuronal Abeta accumulation precedes plaque formation in beta-amyloid precursor protein and presenilin-1 doubletransgenic mice." Neurosci Lett 306(1-2): 116-120.

Wolfe, M. S. (2008). "Inhibition and modulation of gamma-secretase for Alzheimer's disease." Neurotherapeutics 5(3): 391-398.

Woodcock, C. L. and S. Dimitrov (2001). "Higher-order structure of chromatin and chromosomes." Curr Opin Genet Dev 11(2): 130-135.

Xiong, Y., K. Zhao, J. Wu, Z. Xu, S. Jin and Y. Q. Zhang (2013). "HDAC6 mutations rescue human tau-induced microtubule defects in Drosophila." Proc Natl Acad Sci USA 110. 
Xiu, D., L. Liu, F. Qiao, H. Yang, L. Cui and G. Liu (2016). "Annexin A2 Coordinates STAT3 to Regulate the Invasion and Migration of Colorectal Cancer Cells In Vitro." Gastroenterol Res Pract 2016: 3521453.

Yan, G., W. Luo, Z. Lu, X. Luo, L. Li, S. Liu, Y. Liu, M. Tang, Z. Dong and Y. Cao (2007). "Epstein-Barr virus latent membrane protein 1 mediates phosphorylation and nuclear translocation of annexin A2 by activating PKC pathway." Cell Signal 19(2): 341-348.

Yao, Z. G., L. Zhang, L. Huang, H. Zhu, Y. Liu, C. M. Ma, S. L. Sheng and C. Qin (2013). "Regional and cell-type specific distribution of HDAC2 in the adult mouse brain." Brain Struct Funct 218(2): 563-573.

Yu, W. and E. Krook-Magnuson (2015). "Cognitive Collaborations: Bidirectional Functional Connectivity Between the Cerebellum and the Hippocampus." Frontiers in Systems Neuroscience 9: 177.

Yun, M., J. Wu, J. L. Workman and B. Li (2011). "Readers of histone modifications." Cell Res 21(4): 564-578.

Zeng, L. and M. M. Zhou (2002). "Bromodomain: an acetyl-lysine binding domain." FEBS Lett 513(1): 124-128.

Zhang, B., S. Kirov and J. Snoddy (2005). "WebGestalt: an integrated system for exploring gene sets in various biological contexts." Nucleic Acids Res 33(Web Server issue): W741-748.

Zhang, Y., N. Li, C. Caron, G. Matthias, D. Hess, S. Khochbin and P. Matthias (2003). "HDAC-6 interacts with and deacetylates tubulin and microtubules in vivo." The EMBO Journal 22(5): 1168-1179.

Zhang, Z., M. Song, X. Liu, S. S. Kang, I. S. Kwon, D. M. Duong, N. T. Seyfried, W. T. Hu, Z. Liu, J. Z. Wang, L. Cheng, Y. E. Sun, S. P. Yu, A. I. Levey and K. Ye (2014). "Cleavage of tau by asparagine endopeptidase mediates the neurofibrillary pathology in Alzheimer's disease." Nat Med 20(11): 1254-1262.

Zhao, W. Q., D. M. Waisman and M. Grimaldi (2004). "Specific localization of the annexin II heterotetramer in brain lipid raft fractions and its changes in spatial learning." $\mathrm{J}$ Neurochem 90(3): 609-620.

Zheng, L., K. Foley, L. Huang, A. Leubner, G. Mo, K. Olino, B. H. Edil, M. Mizuma, R. Sharma, D. T. Le, R. A. Anders, P. B. Illei, J. E. Van Eyk, A. Maitra, D. Laheru and E. M. Jaffee (2011). "Tyrosine 23 phosphorylation-dependent cell-surface localization of annexin A2 is required for invasion and metastases of pancreatic cancer." PLoS One 6(4): e19390.

Zhou, H. L., M. N. Hinman, V. A. Barron, C. Geng, G. Zhou, G. Luo, R. E. Siegel and H. Lou (2011). "Hu proteins regulate alternative splicing by inducing localized histone hyperacetylation in an RNA-dependent manner." Proc Natl Acad Sci U S A 108(36): E627-635.

Zhou, H. L., G. Luo, J. A. Wise and H. Lou (2014). "Regulation of alternative splicing by local histone modifications: potential roles for RNA-guided mechanisms." Nucleic Acids Res 42(2): 701-713. 


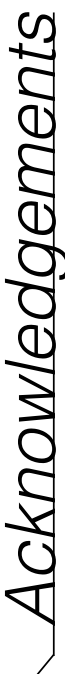




\section{Acknowledgements}

First and foremost I want to sincerely thank Andre for giving me the opportunity to do my PhD in his lab. I also want to thank him for the incredible freedom and support he gave me, for being open to ideas that do not necessarily fall into the scope of his research and for creating a friendly environment in which it was a pleasure to carry out my work for this thesis.

Also I want to thank Prof. Dr. Tiago Outeiro for reviewing this thesis and being a critical but always positive member of my thesis committee. Further, I want to thank Prof. Dr. Siegrid Löwel for exciting discussions and honest feedback during my thesis committee meetings. Additionally I want to express my gratitude in advance to all members of my extended examination board Prof. Dr. Ralf Heinrich, Prof. Dr. André Fiala, and Prof. Dr. Martin Göpfert. All of which impacted my studies here in Göttingen starting from the bachelors and continuing through the master programme. I appreciate the gesture of them being present for my defense.

At this point I also want to express my sincere gratitude to the Hans and Ilse Breuer Foundation for their generous support of work.

I want to express my gratitude to Alan Gillman, and Joon Lee for the collaboration on anle138b and for agreeing to incorporate their data into this thesis. Also I want to thank Nadin Zimmermann for the collaboration on the H4K12ac pulldown.

Now to the Fischerlab old and new, it would not have been the same without them and I want to thank everyone making this such a memorable experience.

Particularly I want to express my greatest gratitude and appreciation to Eva. I believe words don't suffice for all she has done as colleague and friend. Some specific things however: I want to thank her for her comments on this thesis, for being Evapedia, for never being (too) annoyed, for keeping the spirits up and for all the crazy time we spent together. Without her I do not believe I would've started this adventure and yes I'm still glad I did! Further, I want to thank Ana She has been a delight as a bench buddy and I am grateful for giving me the opportunity to contribute to her project and of course for her suggestions on this thesis. Also I want to express my thankfulness to Tea for her input on this thesis and for simply making the lab a better place. Speaking of which, I am very grateful to Susi for her amazing work. Somehow it seems like her day simply has more hours than most. Also I want to thank Rashi for her help with the FACS. Aiiiiiii! Magda! I am very grateful for having this mentally person to share my entire time as a $\mathrm{PhD}$ and I want to thank her for keeping my spirits up. Mucho! Then I am very thankful to Henning for helping with my experiments during a time of sickness and caring for my mental health and throwing sweets at me when needed. Also I want to thank Christian for his input on the methods and simply being the relaxed person he is. Vincezo! I thank him for the great job he's doing and his patience with certain projects... one day we'll drink pools of champagne and laugh about it. I want to thank Gaurav for his ambitions in analysis just anything. Further, I want to thank Ulrike and Daniel for their amazing job they are doing and helping wherever they can.

To my friends here in Göttingen. This has been a ride! We spent now almost 10 years together starting from our very first day as bachelor students. You are - without a doubt - the best bunch of crazy people in the world. Thank you for being there and making this the best time.

Franzi, I am so very grateful for having you in my life. Thank you for caring for me and all of your patience and acceptance during the past months and of course your untiring accuracy and input on this thesis.

Last but not least I want to thank my family. Claus, vielen Dank für deine Ratschläge zu dieser Arbeit. Lisa ich bin so unendlich froh dass du seit meinem aller ersten Tag da bist. Danke für deine Unterstützung, dein Verständnis wenn es mal wieder drunter und drüber ging. Das wichtigste zu guter Letzt. Ich möchte meiner Mutter danken. Danke für all deine Güte, deine Gedult mit mir und dein Verständnis für die gesammte Zeit. Vielen Dank für unseren philosophischen Austausch und einfach dafür, dass es dich gibt. Mit Sicherheit wäre ich ohne deine Unterstützung niemals so weit gekommen. Danke. 


\section{Curriculum Vitae}

PERSONAL

$\begin{array}{ll}\text { Name: } & \text { Hendrik Urbanke } \\ \text { Date of birth: } & 09 / 06 / 1987 \\ \text { Place of birth: } & \text { Nürnberg, Germany }\end{array}$

EDUCATION

Since 01/2014 German Center for Neurodegenerative Diseases

(DZNE), Göttingen

- Ph.D. student, work group 'Epigenetics of Neurodegenerative Diseases', Prof. Dr. Andre Fischer

06/2012 - 11/2012 University Göttingen

- Research assistant, work group 'Behavioural Molecular Neurobiology', Prof. Dr. André Fiala, Schwann-Schleiden-Forschungszentrum, establishing the basis to investigate laterality in D. melanogaster

10/2011 - 10/2013 Georg- August- University Göttingen

- Master of Science in developmental-, neural- and behavioural biology, with distinction, focus on molecular neuroscience

10/2008 - 09/2011 Georg- August- University Göttingen

- Bachelor of Science, biology, focus on neuroscience and morphology

09/1998 - 06/2007 Scheffel- Gymnasium Bad Säckingen

- Abitur

\section{HONORARY OFFICE}

09/2014 - 11/2016 PhD Student Representative DZNE - Göttingen

\section{AWARDS \& FELLOWSHIPS}

2016

"Innovation to Application Award" for inventing and engineering of a method and interface. The complementary developed assay allows precise temporal stimulation using optogenetics and sequencing of single cells depending on their function in a heterogenous cellular network.

Since 01/2015 PhD fellowship of the Hans und Ilse Breuer Alzheimer foundation for outstanding young scientists. 


\section{PUBLICATIONS}

2017

2015

"The diphenylpyrazole compound anle138b blocks $A \beta$ channels and rescues disease phenotypes in a mouse model for amyloid pathology"

Martinez Hernandez $A^{*}$, Urbanke $H^{*}$, Gillman $A^{*}$, Lee $J^{*}$, Ryazanov $S$, Agbemenyah HY, Benito E, Jain G, Kaurani L, Grigorian G, Leonov A, Rezaei-Ghaleh N, Wilken P, Arce FT, Wagner J, Fuhrman M, Caruana M, Camilleri A, Vassallo N, Zweckstetter M, Benz R, Giese A, Schneider A, Korte M, Lal R, Griesinger C, Eichele G, Fischer A. EMBO Mol Med. December 2017; *equal author contribution

"The BET/BRD inhibitor JQ1 improves brain plasticity in WT and APP mice" Benito E, Ramachandran B, Schroeder H, Schmidt G, Urbanke H, Burkhardt S, Capece V, Dean C, Fischer A. Transl Psychiatry. September 2017

"KMT2A and KMT2B Mediate Memory Function by Affecting Distinct Genomic Regions" Kerimoglu C, Sakib MS, Jain G, Benito E, Burkhardt S, Capece V, Kaurani L, Halder R, Agís-Balboa RC, Stilling R, Urbanke H, Kranz A, Stewart AF, Fischer A. Cell Rep. July 2017

"HDAC inhibitor-dependent transcriptome and memory reinstatement in cognitive decline models"

Benito E, Urbanke H, Ramachandran B, Barth J, Halder R, Awasthi A, Jain G, Capece V, Burkhardt S, Navarro-Sala M, Nagarajan S, Schütz AL, Johnsen SA, Bonn S, Lührmann R, Dean C, Fischer A. J Clin Invest. September 2015

"K-Lysine acetyltransferase 2a regulates a hippocampal gene expression network linked to memory formation"

Stilling RM, Rönicke R, Benito E, Urbanke H, Capece V, Burkhardt S, Bahari-Javan S, Barth J, Sananbenesi F, Schütz AL, Dyczkowski J, Martinez-Hernandez A, Kerimoglu C, Dent SY, Bonn S, Reymann KG, Fischer A. The EMBO Journal July 2014 